\title{
QUANTUM TELEPORTATION BETWEEN DISTANT MATTER QUBITS
}

\author{
by \\ Steven Matthew Olmschenk
}

A dissertation submitted in partial fulfillment of the requirements for the degree of

Doctor of Philosophy (Physics)

in The University of Michigan

2009

Doctoral Committee:

Associate Professor Luming Duan, Co-Chair

Professor Christopher R. Monroe, University of Maryland, Co-Chair

Professor Georg A. Raithel

Professor Roseanne J. Sension

Assistant Professor Jennifer P. Ogilvie 
"...I predict that within 100 years, computers will be twice as powerful, 10,000 times larger, and so expensive that only the five richest kings of Europe will own them."

-Professor Frink, The Simpsons 
(C) Steven Matthew Olmschenk All Rights Reserved

2009 
To Sarah. 


\section{ACKNOWLEDGEMENTS}

The years I have spent in graduate school have been instructive, productive, exciting and fun. My experience has been this positive due to the fantastic people I have worked with along the way, and whose efforts and collaborations are embedded in this thesis. Physics is best undertaken in a group, and I have been fortunate to be part of an incredible one.

Chris Monroe has to be the greatest thesis advisor ever. He is incredibly skilled in the lab, and an astonishing source of physics knowledge and intuition. Nevertheless, he grants a great deal of independence in research. Lessons learned from my stumbles and successes in the experiment, coupled with Chris' guidance and expertise, has made everything in this thesis possible. I am very thankful to have had the opportunity to learn from and work with Chris.

Luming Duan has also been crucial to the success of this work. From his instruction in my first semester of graduate school to his close collaboration with the experiment, I have benefited greatly from his knowledge and expertise.

When I first joined the lab, I had the pleasure of working on advanced ion trap structures with Winni Hensinger, Dan Stick, Louis Deslauriers, Dave Hucul, Mark Yeo, and Jim Rabchuk. Particularly important to our productivity was the en-

ergy brought to these experiments by Winni. His stubborn persistence propeled the projects forward, and taught me the proper way to deal with troublesome companies. The research was always exciting and fun, even during the most laborous endeavors. 
After completion of the microfabricated trap, T-trap, and needle trap projects, the focus of my research changed dramatically as I joined the ion-photon entanglement project, which is the focus of this thesis. My introduction to this effort occurred at the crucial point of switching from cadium to ytterbium. As such, I had the opportunity to construct a new lab from an empty room, with Peter Maunz, Dzmitry Matsukevich, David Moehring, and Kelly Younge. During this period of construction, I learned an incredible amount of physics. Peter is an amazing scientist, and I thank him for his patience, guidance, and expertise throughout this research. He has taught me an extraordinary amount of experimental physics, and to never accept "nonsense" in the experiment. Dzmitry has an incredible grasp of atomic physics. Whenever I would get frustrated or confused, it was always nice to be reminded by Dzmitry that it was really quite "easy". David and Kelly were great lab partners, on whose perseverance and effort I always relied. While reconstructing the lab after moving to Maryland, Peter, Dzmitry, and I were joined by Dave Hayes. Dave's background in quantum information theory has already proven to be invaluable, and his efforts in the experiment were crucial to its success.

While I may not have directly worked with all of the members of Chris' lab, I have certainly benefited from their presence. I would therefore like to extend my thanks to Mark Acton, Boris Blinov, Kathy-Anne Brickman, Wes Campbell, MingShien Chang, Andrew Chew, Dan Cook, Brian Fields, Paul Haljan, Rajibul Islam, Kihwan Kim, Rudy Kohn, Simcha Korenblit, Patty Lee, Le Luo, Martin Madsen, Andrew Manning, Jonathan Mizrahi, Liz Otto, Qudsia Quraishi, Yisa Rumala, and Jon Sterk.

Of course, none of my efforts in graduate school would have been possible without my family and friends, whom I cannot possibly thank enough. First and foremost, 
I need to thank my parents for their guidance and love. At an early age, my mom and dad instilled in me a relentless curiosity about the way the world works, and the tenacity needed to accomplish my goals. My parents always supported my endeavors, and were always there whenever and wherever I needed them. I would also like to thank my brothers, Dan, Greg, and Tony, for our close relationship, and for always being there to help me. I thank my Grandma, Grandpa O, and Uncle Mike for their encouragement and love. In addition, I would like to thank the family I married into, Larry, Margaret, and Matthew, for their love and support.

Finally, I will try to express my gratitude to my best friend, my wife, Sarah. She has been a constant source of love and support during this arduous process. In addition to enduring my long hours of lab work, she accepted our move from Michigan to Maryland with incredible fortitude. She continues to be a source of inspiration, encouragement, and love. Sarah, to you I dedicate both this thesis, and my heart. 


\section{TABLE OF CONTENTS}

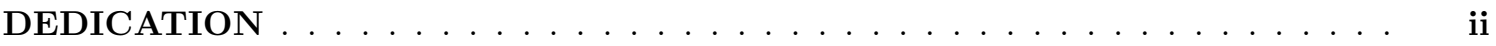

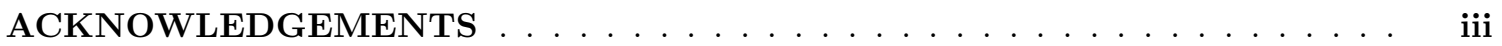

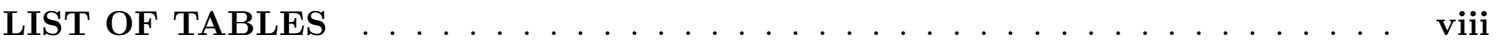

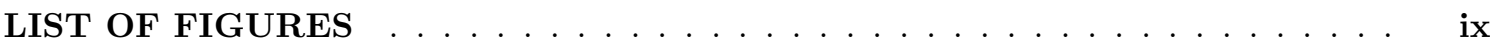

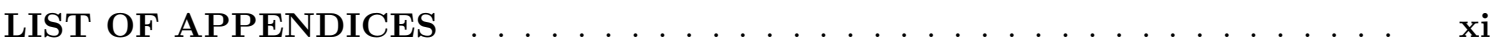

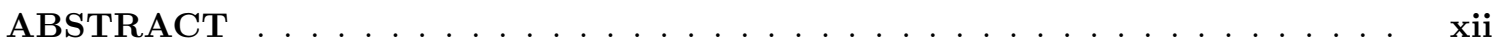

\section{CHAPTER}

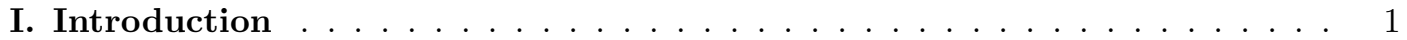

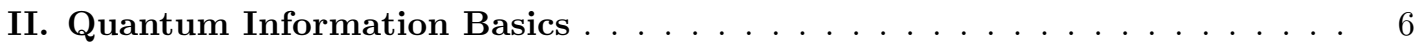

2.1 Quantum computation requirements . . . . . . . . . . . . . 6

2.2 Quantum no-cloning theorem . . . . . . . . . . . . . . . . . 10

2.3 Quantum teleportation ................... 11

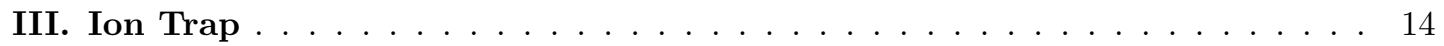

3.1 Ion trap basics . . . . . . . . . . . . . . . . . . . . . . 14

3.2 Trap simulations . . . . . . . . . . . . . . . . . . . . . . . . . . . . . . . . . . . 34

3.3 Vacuum chamber and trap construction . . . . . . . . . . . . . . 37

IV. Ytterbium Ions $\ldots \ldots \ldots \ldots \ldots \ldots \ldots$

4.1 Photoionization . . . . . . . . . . . . . . . . . . . . 48

4.2 Doppler cooling . . . . . . . . . . . . . . . . . . . . . . . . . . . . . . 49

4.3 Laser stabilization . . . . . . . . . . . . . . . . . . . . 55

4.4 Branching ratio of the ${ }^{2} P_{1 / 2}$ level . . . . . . . . . . . . . . . 59

4.5 Hyperfine qubit . . . . . . . . . . . . . . . . . . . 62

$4.6{ }^{2} D_{3 / 2}$ and ${ }^{3}[3 / 2]_{1 / 2}$ hyperfine splittings . . . . . . . . . . . . . . . . . . 77

V. Photon Interference $\ldots \ldots \ldots \ldots \ldots$. . . . . . . . . . . . 80

5.1 Theoretical interference of photons at a beamsplitter . . . . . . . . . 80

5.2 Experimental interference of photons at a beamsplitter . . . . . . . . . 94

VI. Lifetime of the ${ }^{2} P_{1 / 2}$ level in $\mathbf{Y b}^{+} \ldots \ldots \ldots \ldots$. . . . . . . . . . . 102 
6.1 Lifetime measurement . . . . . . . . . . . . . . . . . . 103

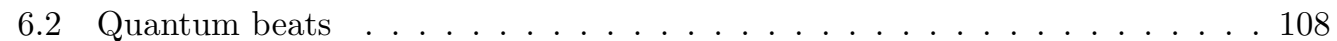

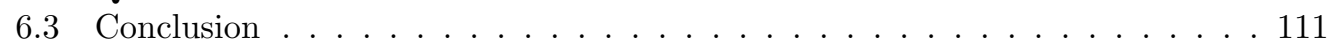

VII. Quantum Gate . . . . . . . . . . . . . . . . . . . . . . . . 114

7.1 Ion-photon entanglement . . . . . . . . . . . . . . . . . . 116

7.2 Ion-ion entanglement . . . . . . . . . . . . . . . . . . . . . 119

7.3 Gate evaluation . . . . . . . . . . . . . . . . . . 123

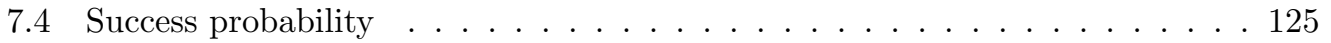

VIII. Teleportation . . . . . . . . . . . . . . . . . . . . . . . 126

8.1 Teleportation protocol . . . . . . . . . . . . . . . . 127

8.2 Experimental evaluation . . . . . . . . . . . . . . . . . 130

8.3 Error analysis . . . . . . . . . . . . . . . . . . . . 132

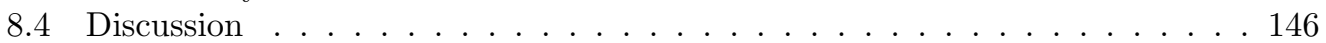

IX. Conclusions and Outlook . . . . . . . . . . . . . . . . . . . . . . . . 148

9.1 Quantum gate with infrared photons . . . . . . . . . . . . . . 148

9.2 Scalability ... . . . . . . . . . . . . . . . . 152

9.3 Summary . . . . . . . . . . . . . . . . . . . 154

APPENDICES .............................. 156

BIBLIOGRAPHY . . . . . . . . . . . . . . . . . . . . . . . 185 


\section{LIST OF TABLES}

\section{Table}

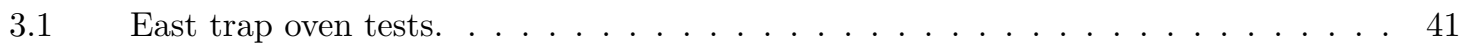

$3.2 \quad$ East trap bake schedule. . . . . . . . . . . . . . . . . . . 43

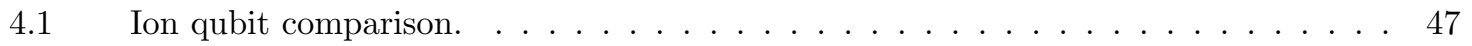

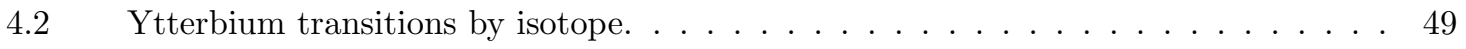

4.3 Theoretical state detection fidelity $\ldots \ldots \ldots \ldots \ldots \ldots$

7.1 Characterization of the heralded quantum gate . . . . . . . . . . . . . 122 


\section{LIST OF FIGURES}

\section{$\underline{\text { Figure }}$}

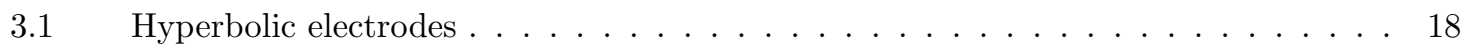

$3.2 \quad$ Motion of a trapped charged particle $\ldots \ldots \ldots \ldots \ldots \ldots \ldots$

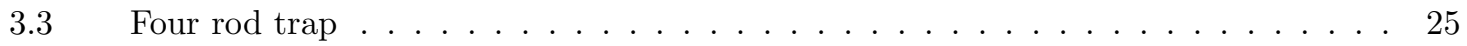

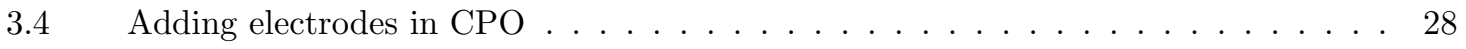

$3.5 \quad$ Adjusting symmetry and voltages in $\mathrm{CPO} \ldots \ldots \ldots \ldots$

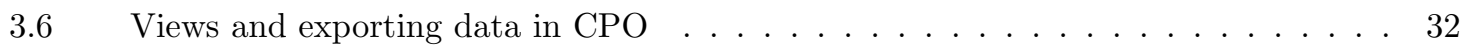

$3.7 \quad$ Numerical simulation of four-rod trap potentials . . . . . . . . . . . . . . . 35

$3.8 \quad$ Vacuum chamber schematic . . . . . . . . . . . . . . . . . . 39

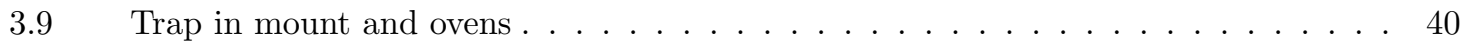

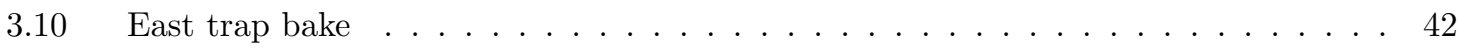

3.11 Helical resonator . . . . . . . . . . . . . . . . . . . . 45

$4.1 \quad$ Neutral $\mathrm{Yb}$ levels . . . . . . . . . . . . . . . . . . . . . . . 50

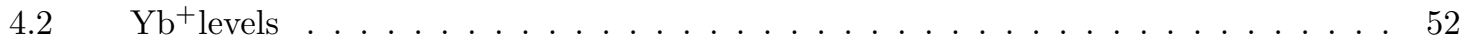

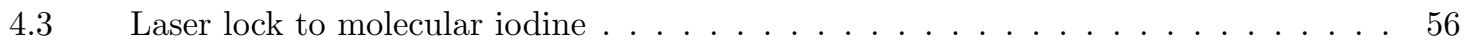

$4.4 \quad$ Hyperfine structure of iodine $\ldots \ldots \ldots \ldots \ldots \ldots \ldots \ldots$

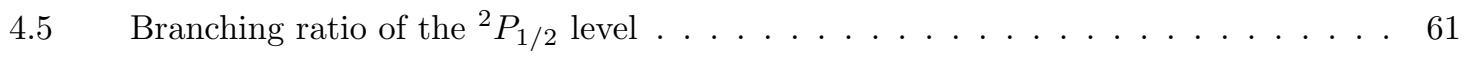

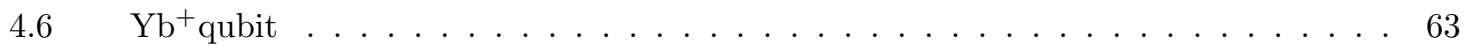

$4.7 \quad$ State detection experiment setup $\ldots \ldots \ldots \ldots \ldots \ldots$

$4.8 \quad$ Dark versus bright histogram $\ldots \ldots \ldots \ldots \ldots \ldots$

4.9 Theoretical Rabi oscillations with detuning $\ldots \ldots \ldots \ldots \ldots \ldots$

4.10 Rabi oscillations with microwaves $\ldots \ldots \ldots \ldots \ldots \ldots$ 
4.11 Coherence time measurement of the ${ }^{171} \mathrm{Yb}^{+}$qubit . . . . . . . . . . . . 78

5.1 Schematic of 50:50 beamsplitter . . . . . . . . . . . . . . . . . 81

5.2 Theoretical single photon first-order correlation function . . . . . . . . . . . . 86

5.3 Theoretical single photon second-order correlation function . . . . . . . . . . . 89

5.4 Theoretical two-photon second-order correlation function . . . . . . . . . . . . 95

5.5 Photon interference experiment setup . . . . . . . . . . . . . . . . . 97

$5.6 \quad$ Picosecond Rabi flopping . . . . . . . . . . . . . . . . . . . . . . 98

5.7 Experimental single-photon second-order correlation function . . . . . . . . . 100

5.8 Experimental two-photon second-order correlation function . . . . . . . . . . 101

6.1 Experimental setup for lifetime measurement . . . . . . . . . . . . . . . 104

6.2 Lifetime data ........................... . . . . . . . . . . . . . . . . . .

6.3 Theoretical quantum beats . . . . . . . . . . . . . . . . 109

6.4 Single-photon second-order correlation function with three missing peaks . . . . . 112

6.5 Comparison with previous values of the $6 p^{2} P_{1 / 2}$ lifetime . . . . . . . . 113

$7.1 \quad$ Heralded gate experiment setup . . . . . . . . . . . . . . . . 116

7.2 Pulsed excitation for ion-photon entanglement . . . . . . . . . . . . . . 118

7.3 State tomography of $\left|\psi^{-}\right\rangle_{\text {ions }} \ldots \ldots \ldots \ldots$. . . . . . . . . . . . . . 124

8.1 Tomography of teleported quantum states . . . . . . . . . . . . . 131

8.2 Process tomography of the teleportation protocol . . . . . . . . . . . . 133

9.1 Quantum gate protocols with infrared transitions . . . . . . . . . . . . 150

C.1 Invar cavity assembly diagram. . . . . . . . . . . . . . . . . . . . 166

C.2 Invar cavity dimensions. . . . . . . . . . . . . . . . . . . . . . . 167

D.1 Radiation patterns of $\pi$ and $\sigma$ transitions . . . . . . . . . . . . . . . . 169

D.2 Angular distribution of probability of detecting $\mathbf{X}_{11}$ radiation . . . . . . . . 173

D.3 Error in the discrimination of photon polarizations. . . . . . . . . . . . . . . 177

E.1 Measured phase of the entangled state . . . . . . . . . . . . . . . . . 184 


\section{LIST OF APPENDICES}

\section{$\underline{\text { Appendix }}$}

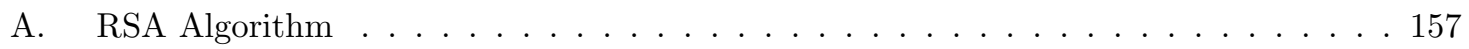

A.1 Number Theory Basics . . . . . . . . . . . . . . . . . . . . . . . . . 157

A.2 Mathematics of Public-Key Cryptography . . . . . . . . . . . . . . 159

A.3 RSA Today . . . . . . . . . . . . . . . . . . . . 160

B. Simulation Analysis in Mathematica . . . . . . . . . . . . . . . . . . 162

C. Reference Cavity . . . . . . . . . . . . . . . . . . . . 165

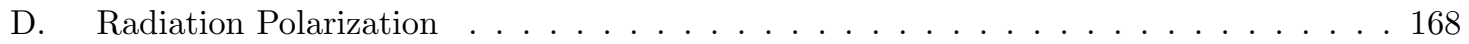

D.1 Radiation from $\Delta \ell=1, \Delta m=0$ transitions . . . . . . . . . . . 169

D.2 Radiation from $\Delta \ell=1, \Delta m=1$ transitions . . . . . . . . . . . . 172

D.3 Implications for Polarization-Encoded Photons . . . . . . . . . . . . . 175

D.4 Implications for Frequency-Encoded Photons . . . . . . . . . . . . . . 178

D.5 Other Considerations . . . . . . . . . . . . . . . . 178

E. Phase of the Gate . . . . . . . . . . . . . . . . . . . . . 179 


\begin{abstract}
QUANTUM TELEPORTATION BETWEEN DISTANT MATTER QUBITS

by

Steven Matthew Olmschenk
\end{abstract}

Co-Chairs: Christopher R. Monroe and Luming Duan

Quantum information research is driven by the prospect of using the features of quantum physics to tackle otherwise intractable computational problems. Systems of trapped atomic ions have proven to be one of the most promising candidates for the realization of quantum computation due to their long trapping times, excellent coherence properties, and exquisite control of the internal atomic states. Integrating ions (quantum memory) with photons (distance link) offers a unique path to large-scale quantum computation and long-distance quantum communication. In this work, I present the implementation of a heralded photon-mediated quantum gate between remote ions, and the employment of this gate to perform a teleportation protocol between two ions separated by a distance of about one meter. A quantum bit stored in the hyperfine levels of a single ytterbium ion $\left(\mathrm{Yb}^{+}\right)$is teleported to a second $\mathrm{Yb}^{+}$atom with an average fidelity of $90 \%$ over a replete set of states. The method demonstrated here avoids many of the issues associated with previously demonstrated motional gates, while presenting a new set of challenges and possibilities for integration to larger systems. Ultimately, this teleportation pro- 
tocol could form the elementary constituent of a long-distance quantum repeater. Moreover, this heralded quantum gate could be used to establish large entangled states for measurement-based quantum computation. 


\section{CHAPTER I}

\section{Introduction}

"Begin at the beginning," the King said, gravely, "and go on till you come to the end: then stop."

-Lewis Carroll, Alice's Adventures in Wonderland

Quantum information research has the potential to drastically alter the fields of communication and computation. Efforts in quantum computation are driven by the prospect of using the features of quantum physics to tackle otherwise intractable computational problems. It was realized early on that controllable quantum systems may be used simulate larger quantum systems far more efficiently than is possible using conventional computers [1], and that individual quantum systems might be used as quantum bits (qubits) for information processing [2]. While both of these instances were crucial points, interest in quantum information increased dramatically in 1994 when Peter Shor unveiled an algorithm that could be implemented on a quantum computer that would enable an exponential speed-up in the factorization of large numbers [3]. Given that current encryption techniques, such as the RSA algorithm (App. A), rely on the relative inability of a conventional computer to factor large numbers, Shor's factorization algorithm linked quantum computation to the immediate and important issue of code-breaking.

On the other hand, quantum information research has also yielded a new method of secure communication, where the security of the encrypted information is guar- 
anteed not by a lack of efficient mathematical or computational algorithms, but by the physical quantum properties of the information carriers. It has been shown that entangled pairs of qubits can be used to securely transfer information, where the presence of an eavesdropper is immediately detected by the measurements made during the protocol $[4,5]$. The essential component for this protocol is the quantum no-cloning theorem (Sec. 2.2), which proves quantum physics prohibits the cloning (copying) of an unknown quantum state [6]. Thus, by using a quantum communication protocol with pairs of correlated qubits, the presence of an eavesdropper measuring the transmitted qubit destroys the correlations, thereby announcing their presence before any information is transmitted.

Of course, the same features of quantum mechanics that are used to ensure the security of communication also prevent a simple "read-and-send" approach to transmitting quantum information. Any attempt to read or measure a quantum superposition state results in a single answer, and thereby lacks the information needed to reconstruct the probabilities of the quantum superposition. The quantum no-cloning theorem forbids an estimation of the unknown quantum state by simply measuring a large number of identical copies. Nevertheless, a quantum state can still be transferred through the process of quantum teleportation [7]. In the quantum teleportation protocol, a quantum state initially stored in system A can be recovered at system B without ever having traversed the space between the systems. The ability to teleport quantum information is an essential ingredient for long-distance quantum communication and may be a vital component to achieve the exponential processing speed-up promised by quantum computation. The essence of quantum teleportation lies in the non-local correlations, or entanglement, afforded by quantum physics.

Quantum communication and quantum computation both use entanglement as an 
essential resource. Entanglement is the quantum correlations between systems that do not have well-defined individual properties. Mathematically, an entangled state is one that cannot be written as a product of individual quantum states; the state is not separable (for examples, see Sec. 2.1). In addition to being useful for quantum communication and quantum computation, entanglement embodies the counter-intuitive depiction of nature predicted by quantum physics, and allows for explicit experimental tests of quantum theory. Entanglement has been observed in a wide variety of systems, including photons [8], atomic ions [9], superconducting Josephson junctions [10], and neutral atoms in cavities [11], confined by an optical lattice [12], and in small ensembles [11]. Hybrid systems composed of a single photonic qubit and a matter qubit (ion [13], ensemble [14], or atom [15]) have also shown entanglement. Here, we will focus on photons and atomic ions, both of which have already proven amenable to applications in quantum information.

Atomic ions are one of the most promising systems for quantum information processing due to their long trapping times, excellent coherence properties, and exquisite control of the internal atomic states. To date, the largest entangled states of individually addressable qubits has been an 8-particle W-state [16] and a 6-particle GHZ state [17], both of which were realized in systems of trapped atomic ions and used the collective motion of the confined atoms to implement the entangling protocol. Effort is now focused on scaling this system to larger numbers of qubits. One approach to scaling in this system [18], which was the focus of my earlier research, is to use microfabricated ion traps [19, 20] and advanced ion trap arrays [21, 22]. Since these deterministic gate operations utilize the common modes of motion of the trapped ions, an understanding of motional decoherence is also actively being pursued $[23,24,25]$. 
Photons, on the other hand, are a natural choice of qubit for communication purposes, as they can quickly traverse the distance between locations with only small perturbations to the encoded quantum information. Already, a series of seminal experiments have used photons in quantum communication protocols over distances as large as $144 \mathrm{~km}$ [26]. Ultimately, though, the direct communication of quantum information over long-distances is impeded by the attenuation of light traveling through optical fibers; even at telecom wavelengths, which experience the least attenuation in fiber, the probability of transmitting a single photon over $1000 \mathrm{~km}$ is $<10^{-20}$. Current fiber-optic information transfer mitigates the loss in signal amplitude by introducing repeaters along the transmission path to "boost" the signal along the way. Although application of the standard model of a repeater to quantum information is prohibited by the no-cloning theorem, an analogous "quantum repeater" has been suggested to enable the transfer of quantum information over arbitrary distances $[27,28]$. In this method, the distance between two points is broken up into a series of shorter segments, with a quantum memory at each connection point, or node. Entanglement can then be established between pairs of nodes, and subsequent segments connected via entanglement swapping, which is used to extend the entanglement over the entire length of the repeater. The final step is to use this long-distance entanglement as a resource to transfer quantum information over that distance by the process of quantum teleportation.

Integrating atomic ions (quantum memory) with photons (distance link) offers a unique path to large-scale quantum computation and long-distance quantum communication. Combining ions with photons enables long-distance quantum operations between stable quantum memories. These photon-mediated operations are insensitive to the motional state of the ions, and thus are unaffected by motional heating 
and do not require ground-state cooling. Moreover, the two-photon scheme presented here is not interferometrically sensitive to the optical path length difference. Large-scale implementation may also be simplified by avoiding the need for complex trap arrays that allow for shuttling ions. In addition, since these operations are mediated by photons, it may be possible to create hybrid matter quantum systems: interfacing atoms with solid-state qubits, such as quantum dots or NV centers. Finally, while this photon-mediated operation is inherently probabilistic, it can still be efficiently scaled to enable generation of the large entangled states required for quantum information processing.

In the following chapters, I present the experimental implementation of probabilistic quantum gate, and employment of this gate in a quantum teleportation protocol to transfer a qubit between two atomic ions separated by one meter. We begin in Chapter II by overviewing some basics of quantum information, including the criteria for universal quantum computation. As indicated above, we have chosen to perform these experiments using photons and trapped atomic ions, so in Chapter III we explore the basics of trapping charged particles, and the construction of our ion trap system. Next we delve into the specifics of our atomic qubit, the ytterbium ion $\left(\mathrm{Yb}^{+}\right)$, in Chapter IV. Chapter $\mathrm{V}$ presents the two-photon interference effect. In Chapter VII are the results of the experimental implementation of the heralded quantum gate. Finally, Chapter VIII illustrates the use of this gate in the long-distance teleportation protocol. Chapter IX concludes with an overview of the potential practical application of these results to long-distance quantum communication and large-scale quantum computation. 


\title{
CHAPTER II
}

\section{Quantum Information Basics}

\begin{abstract}
"There are 10 kinds of people in this world: those who understand binary, and those who don't."

-Anonymous
\end{abstract}

\subsection{Quantum computation requirements}

The quantum bit (qubit) is the basic, fundamental component of a quantum computer. While a binary digit, or bit, in a classical computer only assumes a value of either "0" or "1", quantum physics allows a qubit to be in an arbitrary superposition of states:

$$
|\psi\rangle=\alpha|0\rangle+\beta|1\rangle
$$

where $\alpha$ and $\beta$ are two arbitrary complex numbers that satisfy $|\alpha|^{2}+|\beta|^{2}=1$. These superpositions are what give quantum computation its power. Notice that two classical bits can be in any one of the four possible configurations 00, 01, 10, or 11, but in any configuration is still only two bits of information. Two qubits, on the other hand, can simultaneously be in all four possible states:

$$
|\psi\rangle=\alpha|00\rangle+\beta|01\rangle+\gamma|10\rangle+\delta|11\rangle
$$

In general, $\mathrm{n}$ qubits can be in $2^{\mathrm{n}}$ states simultaneously. The goal of quantum computation is to exploit this massive encoding to perform deterministic information processing. 
Of course, measurement of the quantum state $|\psi\rangle$ will only yield one of the states $|00\rangle,|01\rangle$, $|10\rangle$, or $|11\rangle$, with probability $|\alpha|^{2},|\beta|^{2},|\gamma|^{2}$, and $|\delta|^{2}$, respectively. So at first glance, it would appear that we have not really gained anything! Nevertheless, it turns out that it is possible to capitalize on the large encoding afforded by quantum superpositions by tailoring the computation in such a way that the various superpositions interfere. Interference allows the otherwise probabilistic measurement process to yield a deterministic result. In analogy to current computer science, the operations used to manipulate the qubits and invoke the interferences between them are referred to as quantum gates.

The necessary and sufficient conditions required to implement universal quantum computation protocols have been explicitly stated to be [29]:

- state initialization of the qubits

- long-lived coherences

- universal set quantum gates

- efficient qubit measurment

- scalable to large numbers of qubits

Of the requirements above, perhaps the most opaque is the stipulation of a "universal" set of quantum gates. Basically, this means we require a finite set of operations that can be concatenated to perform any arbitrary operation. It has been shown that a universal set of quantum gates consist of single-qubit gates and a two-qubit controlled-NOT (CNOT) gate [30].

Single qubits gates, restricted to the their two-dimensional Hilbert space, can be 
simply written as combinations of the usual Pauli operators. As matrices, these are:

$$
\hat{\sigma}_{0}=\left(\begin{array}{cc}
1 & 0 \\
0 & 1
\end{array}\right), \hat{\sigma}_{1}=\left(\begin{array}{cc}
0 & 1 \\
1 & 0
\end{array}\right), \hat{\sigma}_{2}=\left(\begin{array}{cc}
0 & -i \\
i & 0
\end{array}\right), \hat{\sigma}_{3}=\left(\begin{array}{cc}
1 & 0 \\
0 & -1
\end{array}\right)
$$

For instance, the action of a $\hat{\sigma}_{1}$ gate is $\hat{\sigma}_{1}|0\rangle=|1\rangle$. A single qubit gate of particular interest is the Hadamard gate, defined as $\hat{H}_{d}=1 /(\sqrt{2})\left(\hat{\sigma}_{1}+\hat{\sigma}_{3}\right)$. The action of the Hadamard gate can be seen from $\hat{H}_{d}|0\rangle=1 /(\sqrt{2})(|0\rangle+|1\rangle)$.

The two-qubit CNOT gate is defined as the operation $\hat{U}_{\text {cnot }}$ such that

$$
\begin{aligned}
& \hat{U}_{\text {cnot }}|00\rangle=|00\rangle \\
& \hat{U}_{\text {cnot }}|01\rangle=|01\rangle \\
& \hat{U}_{\text {cnot }}|10\rangle=|11\rangle \\
& \hat{U}_{\text {cnot }}|11\rangle=|10\rangle
\end{aligned}
$$

which can be succinctly phrased "if the first qubit is $|1\rangle$, flip the second." The process of implementing this two-qubit quantum gate is intimately related to the concept of entanglement. As mentioned in the previous chapter, two systems are entangled if the quantum state is not separable. As an example, the quantum state describing systems $a$ and $b$

$$
|\psi\rangle_{u n}=\frac{1}{\sqrt{2}}\left(|0\rangle_{a}|0\rangle_{b}+|1\rangle_{a}|0\rangle_{b}\right)
$$

is not entangled because it can be factored to

$$
|\psi\rangle_{u n}=\frac{1}{\sqrt{2}}\left(|0\rangle_{a}+|1\rangle_{a}\right)|0\rangle_{b}
$$

and thus a measurement of system $a$ does not impact the result of a measurement on system $b$. On the other hand, the state

$$
|\psi\rangle_{e n}=\frac{1}{2}\left(|0\rangle_{a}|0\rangle_{b}+|1\rangle_{a}|1\rangle_{b}\right)
$$


is entangled. The relation between entanglement and the CNOT gate can be seen by the action of the CNOT gate on the unentangled state of Eq. 2.3.

$$
\begin{aligned}
\hat{U}_{\text {cnot }}|\psi\rangle_{u n} & =\hat{U}_{\text {cnot }} \frac{1}{\sqrt{2}}\left(|0\rangle_{a}|0\rangle_{b}+|1\rangle_{a}|0\rangle_{b}\right) \\
& =\frac{1}{\sqrt{2}}\left(\hat{U}_{\text {cnot }}|0\rangle_{a}|0\rangle_{b}+\hat{U}_{c n o t}|1\rangle_{a}|0\rangle_{b}\right) \\
& =\frac{1}{\sqrt{2}}\left(|0\rangle_{a}|0\rangle_{b}+|1\rangle_{a}|1\rangle_{b}\right)
\end{aligned}
$$

Thus, the CNOT gate transforms the unentangled state $|\psi\rangle_{\text {un }}$ into the entangled state $|\psi\rangle_{\text {en }}$. In light of this relation, entanglement can be viewed as a fundamental resource for quantum information processing.

The subsequent chapters detail how a qubit stored in a single ytterbium ion $\left(\mathrm{Yb}^{+}\right)$satisfies the above criteria necessary for universal quantum computation. In Chap. IV, we demonstrate efficient state initialization through optical pumping, single-qubit gate operations using microwave radiation, long-lived coherences, and high-fidelity measurement of the qubit state through state dependent fluorescence. While the implementation in $\mathrm{Yb}^{+}$is relatively recent, all of these items have been demonstrated previously in other atomic ion species [9]. The focus of this thesis is the implementation of a heralded two-qubit quantum gate, which is an entirely new approach to the trapped ion quantum computer. Ultimately, we use this quantum gate to perform a quantum teleportation protocol between two atoms in independent vacuum chambers, separated by a distance of about one meter. In addition to serving as a demonstration of a simple quantum algorithm, quantum teleportation can be used to transfer fragile quantum information, in spite of the quantum no-cloning theorem. 


\subsection{Quantum no-cloning theorem}

As aforementioned, the quantum no-Cloning theorem prohibits copying an unknown quantum state [6]. Given the relevance of the theorem to the teleportation protocol, we present a short proof below.

Theorem 2.2.1 (Quantum No-Cloning Theorem) If $\left|\psi_{1}\right\rangle$ and $\left|\psi_{2}\right\rangle$ are two nonorthogonal distinct states, there does not exist a quantum evolution (unitary operation) that perfectly copies these two states.

Proof Given that $\left|\psi_{1}\right\rangle$ and $\left|\psi_{2}\right\rangle$ are distinct and nonorthogonal states, then

$$
\left\langle\psi_{1} \mid \psi_{2}\right\rangle=a \neq 0,1
$$

Let's do a proof by contradiction. Suppose there exists a unitary operator $U$ such that

$$
\begin{aligned}
& U\left|\psi_{1}\right\rangle=\left|\psi_{1}\right\rangle\left|\psi_{1}\right\rangle \\
& U\left|\psi_{2}\right\rangle=\left|\psi_{2}\right\rangle\left|\psi_{2}\right\rangle
\end{aligned}
$$

Then,

$$
a=\left\langle\psi_{1} \mid \psi_{2}\right\rangle=\left\langle\psi_{1}|I| \psi_{2}\right\rangle=\left\langle\psi_{1}\left|U^{\dagger} U\right| \psi_{2}\right\rangle=\left\langle\psi_{1}\left|\left\langle\psi_{1} \mid \psi_{2}\right\rangle\right| \psi_{2}\right\rangle=a\left\langle\psi_{1} \mid \psi_{2}\right\rangle=a^{2}
$$

So, we have found

$$
a=a^{2}
$$

which implies,

$$
a=0,1
$$

a contradiction. $\rightarrow \leftarrow$ 
The essential point of the preceding proof is that an unknown quantum state cannot be perfectly copied. As discussed earlier, this result is of fundamental importance for the security of quantum communication protocols, and highlights the importance of quantum teleportation for the transfer of quantum information.

\subsection{Quantum teleportation}

Quantum teleportation was first proposed by Bennett et al. in 1993 [7]. Teleportation aims to utilize a shared entangled state as a means to transfer quantum information. Suppose we need to transfer the unknown quantum state $|\psi\rangle_{t}=\alpha|0\rangle_{a 1}+\beta|1\rangle_{a 1}$ from our site at $a$ to another site, $b$. Since we only have a single copy, we are unable to estimate the probabilities $|\alpha|^{2}$ and $|\beta|^{2}$ through measurement. Moreover, by the quantum no-cloning theorem, we are unable to make this a possibility by generating additional copies of the state. Fortunately, we are able to generate a maximally entangled state between the sites $a$ and $b$, and perform single-qubit gates and measure our two qubits at site $a$ in the Bell state basis; enough to perform quantum teleportation.

The maximally entangled Bell states, also called Einstein-Podolsky-Rosen (EPR) states, are defined as:

$$
\begin{aligned}
\left|\psi^{-}\right\rangle_{i, j} & =\frac{1}{\sqrt{2}}\left(|0\rangle_{i}|1\rangle_{j}-|1\rangle_{i}|0\rangle_{j}\right) \\
\left|\psi^{+}\right\rangle_{i, j} & =\frac{1}{\sqrt{2}}\left(|0\rangle_{i}|1\rangle_{j}+|1\rangle_{i}|0\rangle_{j}\right) \\
\left|\phi^{-}\right\rangle_{i, j} & =\frac{1}{\sqrt{2}}\left(|0\rangle_{i}|0\rangle_{j}-|1\rangle_{i}|1\rangle_{j}\right) \\
\left|\phi^{+}\right\rangle_{i, j} & =\frac{1}{\sqrt{2}}\left(|0\rangle_{i}|0\rangle_{j}+|1\rangle_{i}|1\rangle_{j}\right)
\end{aligned}
$$

where the subscripts $i, j$ denote the two systems. Although the act of measuring in the basis defined by the Bell states can be experimentally challenging, we will just 
assume that we have that capability at site $a$ for now. ${ }^{1}$

The teleportation protocol begins by entangling an auxiliary qubit at $a$ with the qubit at site $b$ set to receive the information. At this time, we will assume we are able to generate the $\left|\psi^{-}\right\rangle_{a, b}$ state between $a$ and $b$. After producing this state, we can write the state of the entire system as:

$$
\begin{aligned}
|\Psi\rangle & =|\psi\rangle_{t}\left|\psi^{-}\right\rangle_{a, b} \\
& =\frac{1}{\sqrt{2}}\left(\alpha|0\rangle_{a 1}+\beta|1\rangle_{a 1}\right)\left[|0\rangle_{a}|1\rangle_{b}-|1\rangle_{a}|0\rangle_{b}\right] \\
& =\frac{1}{\sqrt{2}}\left[\alpha|0\rangle_{a 1}|0\rangle_{a}|1\rangle_{b}+\beta|1\rangle_{a 1}|0\rangle_{a}|1\rangle_{b}-\alpha|0\rangle_{a 1}|1\rangle_{a}|0\rangle_{b}-\beta|1\rangle_{a 1}|1\rangle_{a}|0\rangle_{b}\right]
\end{aligned}
$$

Using Eq. 2.7, we rewrite the states of the qubits at site $a$ in the Bell basis.

$$
\begin{aligned}
|\Psi\rangle= & \frac{1}{\sqrt{2}}\left[\alpha \frac{1}{\sqrt{2}}\left(\left|\phi^{+}\right\rangle_{a 1, a}+\left|\phi^{-}\right\rangle_{a 1, a}\right)|1\rangle_{b}+\beta \frac{1}{\sqrt{2}}\left(\left|\psi^{+}\right\rangle_{a 1, a}-\left|\psi^{-}\right\rangle_{a 1, a}\right)|1\rangle_{b}\right. \\
& \left.-\alpha \frac{1}{\sqrt{2}}\left(\left|\psi^{+}\right\rangle_{a 1, a}+\left|\psi^{-}\right\rangle_{a 1, a}\right)|0\rangle_{b}-\beta \frac{1}{\sqrt{2}}\left(\left|\phi^{+}\right\rangle_{a 1, a}-\left|\phi^{-}\right\rangle_{a 1, a}\right)|0\rangle_{b}\right] \\
= & \frac{1}{2}\left[\left(\alpha|1\rangle_{b}-\beta|0\rangle_{b}\right)\left|\phi^{+}\right\rangle_{a 1, a}+\left(\alpha|1\rangle_{b}+\beta|0\rangle_{b}\right)\left|\phi^{-}\right\rangle_{a 1, a}\right. \\
& \left.+\left(\beta|1\rangle_{b}-\alpha|0\rangle_{b}\right)\left|\psi^{+}\right\rangle_{a 1, a}-\left(\alpha|0\rangle_{b}+\beta|1\rangle_{b}\right)\left|\psi^{-}\right\rangle_{a 1, a}\right]
\end{aligned}
$$

Thus, we can see that if we perform a measurement at site $a$ that allows us to distinguish between the different Bell states, we will project the qubit at site $b$ into one of four possible states:

$$
\begin{aligned}
\left|\phi^{+}\right\rangle_{a 1, a} & \Rightarrow \alpha|1\rangle_{b}-\beta|0\rangle_{b} \\
\left|\phi^{-}\right\rangle_{a 1, a} & \Rightarrow \alpha|1\rangle_{b}+\beta|0\rangle_{b} \\
\left|\psi^{+}\right\rangle_{a 1, a} & \Rightarrow \beta|1\rangle_{b}-\alpha|0\rangle_{b} \\
\left|\psi^{-}\right\rangle_{a 1, a} & \Rightarrow \alpha|0\rangle_{b}+\beta|1\rangle_{b}
\end{aligned}
$$

Of course, our Bell state measurement at site $a$ yields one of these four possible outcomes randomly. Thus, before quantum state can be recovered at site $b$, we need

\footnotetext{
${ }^{1}$ Alternatively, the issue of measuring in the Bell basis can framed as the capability to perform a CNOT gate and single qubit gates [31].
} 
to relay the result of our measurement outcome at $a$. Since we need to communicate one of four possible outcomes, this requires two classical bits of information.

After $b$ knows the result of the measurement at $a$, a simple single qubit gate operation can be performed to recover the state that was initially at $a$. If $\left|\phi^{+}\right\rangle_{a 1, a}$ was measured at $a$, then the operation $i \hat{\sigma}_{2}$ is employed so that $i \hat{\sigma}_{2}\left(\alpha|1\rangle_{b}-\beta|0\rangle_{b}\right)=$ $\alpha|0\rangle_{b}+\beta|1\rangle_{b}$. Similarly, if $\left|\phi^{-}\right\rangle_{a 1, a}$ is measured $\hat{\sigma}_{1}$ is implemented; if $\left|\psi^{+}\right\rangle_{a 1, a}$, then $-\hat{\sigma}_{3}$; and if $\left|\psi^{-}\right\rangle_{a 1, a}$, then $\hat{\sigma}_{0}$ (the identity - nothing needs to be done in this case). This final single qubit operation completes the teleportation protocol.

There are a couple of salient points to take away from the above discussion. First, we required the ability to perform measurement in the Bell basis, a feat which is not yet obvious how to accomplish. Second, the protocol required two bits of classical information transfer. Without this classical communication, the state at $b$ is left in an unknown quantum state, and no information can be gleaned from it. The classical communication requirement ensures that no information is transferred superluminally. Finally, the act of measurement at site $a$ destroys the quantum superposition state, and thus the unknown quantum state is only transferred, not copied, obeying the quantum no-cloning theorem.

Nevertheless, the information was transferred between sites $a$ and $b$ without actually traversing the space between these systems; hence the terminology "teleportation." This non-local information transfer is enabled by the entanglement between the two systems, and exemplifies the counter-intuitive aspects of quantum physics.

With a basic understanding of teleportation and its implications for quantum information, we can now delve into its experimental realization. 


\section{CHAPTER III}

\section{Ion Trap}

\footnotetext{
"If we hit that bullseye, the rest of the dominoes should fall like a house of cards. Checkmate."

-Capt. Zapp Brannigan, Futurama
}

The radiofrequency (rf) ion trap is an essential tool for all of the experiments presented in the following chapters. The rf ion trap was invented by Wolfgang Paul, for which he shared the 1989 Nobel prize [32]. The first laser-cooling experiments with atoms were reported independently by Wineland et al. [33] using $\mathrm{Mg}^{+}$, and Neuhauser et al. [34] using $\mathrm{Ba}^{+} .{ }^{1}$ Since its introduction, the rf ion trap has been used for a wealth of applications, including atomic clocks, measurements of fundamental constants, mass and frequency spectroscopy [35, 36, 37, 38], and, of course, quantum information [9].

\subsection{Ion trap basics}

Earnshaw's Theorem states "a charged particle cannot be held in a stable equilibrium by electrostatic forces alone" [39]. This result is also embodied in the freespace Maxwell equation $\nabla \cdot \hat{E}=0$, which in words states electric field lines entering a region also need to exit the region; thus, any configuration of static fields will always be anti-trapping in some direction. Of course, it is still possible to confine charged

\footnotetext{
${ }^{1}$ The experiment by Wineland et al. used a Penning trap to confine the ions. Subsequent experiments by Wineland et al. have used the type of rf trap presented here.
} 
particles with electromagnetic fields by using either a combination of electric and magnetic fields (Penning trap) or dynamic electric fields (rf or Paul trap). In the following, we concentrate exclusively on the rf trap.

3.1.1 Average force of a dynamic field First we shall briefly review how an oscillating electric force can produce a time-averaged force on a charged particle, following the discussion of Ref. [40]. For simplicity, consider a particle of mass $m$ and charge $e$ residing in an effective homogenous, one-dimensional electric field, such as that produced inside a large parallel-plate capacitor. If the potential difference between the two plates separated by distance $2 d$ is $V_{0} \cos \left(\Omega_{T} t\right)$, then the force exerted on the particle is simply:

$$
\begin{aligned}
F=m \ddot{z} & =e E(z, t) \\
& =\frac{e}{2 d} V_{0} \cos \left(\Omega_{T} t\right) \\
& =e E_{0}(z) \cos \left(\Omega_{T} t\right)
\end{aligned}
$$

where we have written the electric field as a product of its spatial variation $E_{0}(z)$ and its time variation $\cos \left(\Omega_{T} t\right)$. Also assumed is that the plates are parallel to the $x, y$ plane, yielding an electric field in the $z$-direction. By integrating this equation, we find the solution:

$$
z(t)=z_{0}-\frac{e E_{0}(z)}{m \Omega_{T}^{2}} \cos \left(\Omega_{T} t\right)
$$

with the initial conditions $z(0)=z_{0}$ and $\dot{z}(0)=0$. Clearly, the time-average of the force in Eq. 3.1 reveals the average force felt by the particle is zero; there is no net change in the position of the particle.

Now consider a field similar to the previous, except that it is an inhomogenous field - achieved, for example, by bending the plates of the capacitor slightly. Clearly, this will alter the spatial dependence of the field, $E_{0}(z)$, while the time dependance 
remains $\cos \left(\Omega_{T} t\right)$. For only a small perturbation of the field, we expect the position of the particle over short times to be approximately constant. The electric field can then be expanded around the point $z_{0}$ to obtain:

$$
\begin{aligned}
E_{0}(z) & \approx E_{0}\left(z_{0}\right)+\left.\frac{\partial E_{0}(z)}{\partial z}\right|_{z_{0}}\left(z-z_{0}\right) \\
& \approx E_{0}\left(z_{0}\right)+\frac{\partial E_{0}\left(z_{0}\right)}{\partial z_{0}}\left(\frac{e E_{0}\left(z_{0}\right)}{m \Omega_{T}^{2}} \cos \left(\Omega_{T} t\right)\right)
\end{aligned}
$$

where in the last line we have made the approximation that the derivative of $E_{0}\left(z_{0}\right)$ is much smaller than $E_{0}\left(z_{0}\right)$, so that a chain-rule expansion of $\frac{\partial E_{0}(z)}{\partial z}$ yields $\frac{\partial E_{0}\left(z_{0}\right)}{\partial z_{0}}$. With the same approximation, substitution of Eq. 3.2 for $\left(z-z_{0}\right)$ yields the expression shown by neglecting higher order terms.

The equation for the force on the charged particle, Eq. 3.1, then becomes

$$
\begin{aligned}
F & =e E_{0}(z) \cos \left(\Omega_{T} t\right) \\
& \approx e E\left(z_{0}\right) \cos \left(\Omega_{T} t\right)-e \frac{\partial E_{0}\left(z_{0}\right)}{\partial z_{0}}\left(\frac{e E_{0}\left(z_{0}\right)}{m \Omega_{T}^{2}} \cos ^{2}\left(\Omega_{T} t\right)\right)
\end{aligned}
$$

Averaging this over one oscillation yields

$$
\begin{aligned}
\bar{F} & \approx-\frac{e^{2}}{2 m \Omega_{T}^{2}} \frac{\partial E_{0}\left(z_{0}\right)}{\partial z_{0}} E_{0} \\
& =-e \frac{\partial \psi_{p}}{\partial z_{0}}
\end{aligned}
$$

where we have defined the "pseudopotential," $\psi_{p}$, as

$$
\psi_{p}=\frac{e E_{0}^{2}}{4 m \Omega_{T}^{2}}
$$

Thus, we see that for an inhomogenous, oscillating electric field, the time-averaged force on a charged particle does not vanish. Instead, the particle sees an effective potential created by the inhomogenity of the field, resulting in a net force on the particle towards the area of weaker field. Although we only derived this in one 
dimension, it is easily extended.

$$
\bar{F}=-e \nabla \psi_{p} \text { with } \psi_{p}=\frac{e\left(\hat{E}_{0}(x, y, z)\right)^{2}}{4 m \Omega_{T}^{2}}
$$

We can now use Eq. 3.7 to determine the effective motion of a charged particle confined by a trapping field.

3.1.2 Pseudopotential approximation In order to trap charged particles, we are interested in dynamic quadrupole potentials. As an illustration in two dimensions, consider the idealized hyperbolic electrode configuration shown in Fig. 3.1. Since we know the equation for a hyperbola, then given that the left and right electrodes have potential $V_{0} \cos \left(\Omega_{T} t\right)$ applied, while the top and bottom electrodes are held as 0 , then the potential between the electrodes is simply given by

$$
\phi_{\text {hyp }}=\frac{V_{0}}{2} \cos \left(\Omega_{T} t\right)\left(1+\frac{x^{2}-y^{2}}{R^{2}}\right)
$$

which clearly satisfies the boundary conditions, and Laplace's equation.

The electric field produced by this potential is

$$
\begin{aligned}
\hat{E}(x, y, t) & =-\nabla \phi_{\text {hyp }} \\
& =-\frac{V_{0}}{R^{2}}(x \hat{x}-y \hat{y}) \cos \left(\Omega_{T} t\right) \\
& =-\hat{E}_{0}(x, y) \cos \left(\Omega_{T} t\right)
\end{aligned}
$$

Plugging the expression for $\hat{E}_{0}(x, y)$ defined by Eq. 3.9 into Eq. 3.7, we obtain

$$
\begin{aligned}
\bar{F} & =-e \frac{\partial}{\partial x}\left(\frac{e V_{0}^{2} x^{2}}{4 m \Omega_{T}^{2} R^{4}}\right) \hat{x}-e \frac{\partial}{\partial y}\left(\frac{e V_{0}^{2} y^{2}}{4 m \Omega_{T}^{2} R^{4}}\right) \hat{y} \\
& =-\frac{e^{2} V_{0}^{2}}{2 m \Omega_{T}^{2} R^{4}} x \hat{x}-\frac{e^{2} V_{0}^{2}}{2 m \Omega_{T}^{2} R^{4}} y \hat{y}
\end{aligned}
$$

Looking at just the equation of motion for $x$, we find,

$$
\ddot{x}=-\frac{e^{2} V_{0}^{2}}{2 m^{2} \Omega_{T}^{2} R^{4}} x
$$




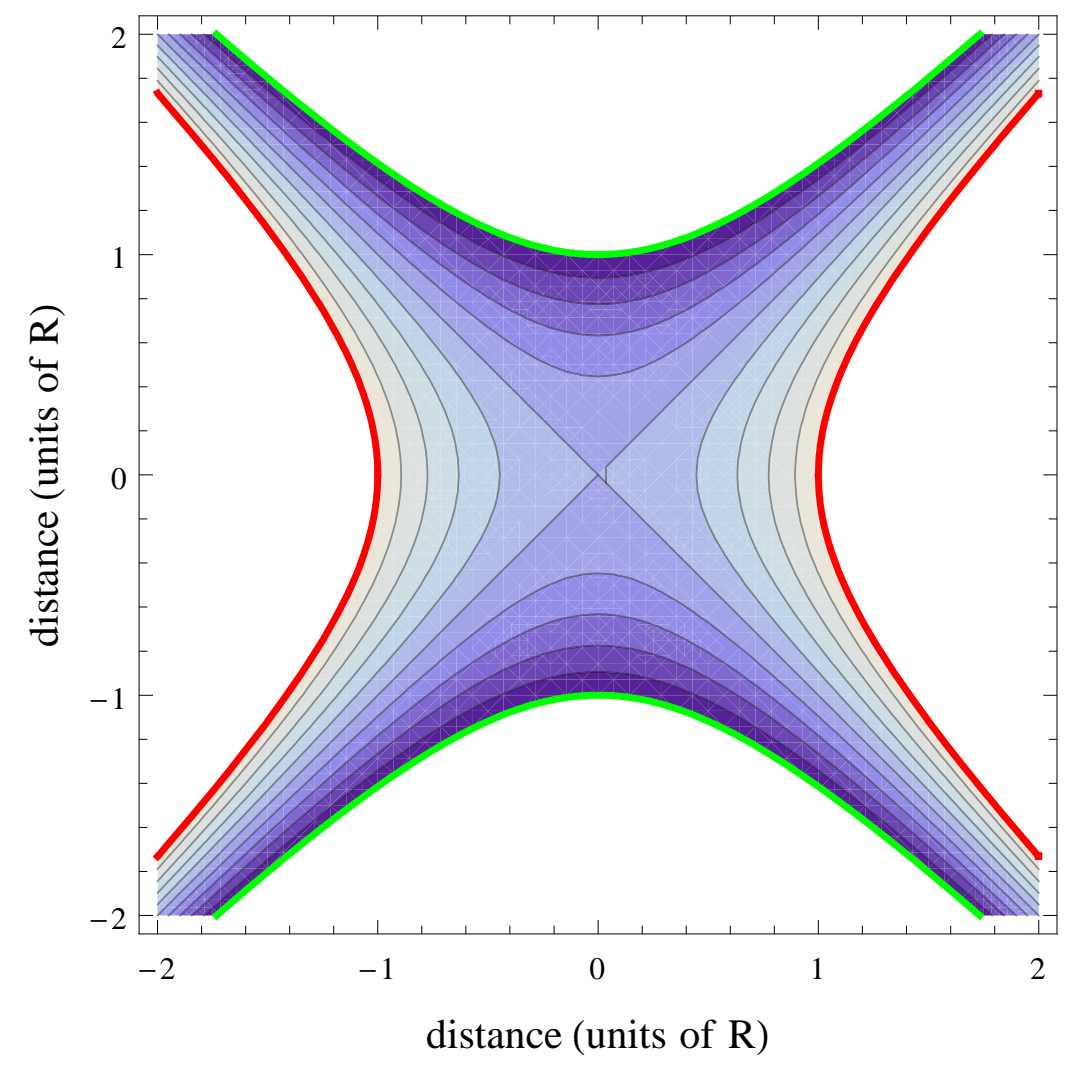

Figure 3.1: Hyperbolic electrodes. This two-dimensional view shows ideal hyperbolic electrodes where the green electrodes are held at ground, and the red electrodes are at $V_{0} \cos \left(\Omega_{T} t\right)$. The resulting potential is shown as a contour plot between the electrodes (shades of blue) for $t=0$. 
which is just the equation for a harmonic oscillator with frequency

$$
\omega_{x}=\frac{e V_{0}}{2^{1 / 2} m \Omega_{T} R^{2}}
$$

This oscillation is called the "secular motion" of the ion. The result for $y$ is exactly the same. Thus, we see that a charged particle near the center of an oscillating quadrupole field experiences an effective harmonic potential, which traps the charged particle.

3.1.3 Mathieu equation While the previous section presented a nice introduction to trapping charged particles with dynamic electric fields, we will now seek a more complete solution. As before, we will assume hyperbolic electrodes in the $x, y$-plane that produce the potential given by Eq. 3.8, and the electric field shown in Eq. 3.9. The force in the $x$-direction on a particle of mass $m$ and charge $e$ is then

$$
F_{x}=m \ddot{x}=-\frac{e V_{0}}{R^{2}} \cos \left(\Omega_{T} t\right) x
$$

yielding the equation of motion

$$
\ddot{x}+\frac{e V_{0}}{m R^{2}} \cos \left(\Omega_{T} t\right) x=0
$$

This is actually just a simplified version of a Mathieu equation. The general form of the Mathieu equation is:

$$
\frac{d^{2} u}{d \tau^{2}}+\left(a_{u}+2 q_{u} \cos (2 \tau)\right) u=0
$$

Our Eq. 3.14 can be put in this form by making the substitution $2 \tau=\Omega_{T} t$. By the chain rule, we then have $\frac{d}{d t}=\frac{d}{d \tau} \frac{d \tau}{d t}=\frac{\Omega_{T}}{2} \frac{d}{d \tau}$, so that Eq. 3.14 can be written

$$
\frac{d^{2} x}{d \tau^{2}}+2 q_{x} \cos (2 \tau) x=0
$$

where in the last line we have defined $q_{x}=\left(2 e V_{0}\right) /\left(m R^{2} \Omega_{T}^{2}\right)$. Note that compared to the general form of Eq. 3.15, here we have $a_{x}=0 .^{2}$

\footnotetext{
${ }^{2}$ In the present case, we have neglected the third dimension of the problem. The ion can be confined in the third dimension using static potentials, in which case $a_{x} \neq 0$; a good review is Ref. [41].
} 
The Floquet Theorem suggests a general solution of the form [42]

$$
x(\tau)=A \sum_{n=-\infty}^{\infty} C_{2 n} \cos ((2 n+\beta) \tau)+i B \sum_{n=-\infty}^{\infty} C_{2 n} \sin ((2 n+\beta) \tau)
$$

where $A, B$ are determined by the initial conditions. We can solve for $\beta$ and the coefficients $C_{2 n}$ by plugging Eq. 3.17 back into Eq. 3.16. We then find

$$
\begin{gathered}
A \sum_{n=-\infty}^{\infty} C_{2 n}(2 n+\beta)^{2} \cos ((2 n+\beta) \tau) \\
+i B \sum_{n=-\infty}^{\infty} C_{2 n}(2 n+\beta)^{2} \sin ((2 n+\beta) \tau) \\
=2 q_{x} A \sum_{n=-\infty}^{\infty} C_{2 n} \cos (2 \tau) \cos ((2 n+\beta) \tau) \\
+i 2 q_{x} B \sum_{n=-\infty}^{\infty} C_{2 n} \cos (2 \tau) \sin ((2 n+\beta) \tau)
\end{gathered}
$$

Using the trigonometric product-to-sum relation for $\operatorname{cosine}{ }^{3}$, we get

$$
\begin{aligned}
& A \sum_{n=-\infty}^{\infty} C_{2 n}(2 n+\beta)^{2} \cos ((2 n+\beta) \tau) \\
& +i B \sum_{n=-\infty}^{\infty} C_{2 n}(2 n+\beta)^{2} \sin ((2 n+\beta) \tau) \\
= & q_{x} A \sum_{n=-\infty}^{\infty} C_{2 n}(\cos ((2 n+\beta) \tau+2 \tau)+\cos ((2 n+\beta) \tau-2 \tau)) \\
& +i 2 q_{x} B \sum_{n=-\infty}^{\infty} C_{2 n} \cos (2 \tau) \sin ((2 n+\beta) \tau) \\
= & q_{x} A \sum_{n=-\infty}^{\infty} C_{2 n-2} \cos ((2 n+\beta) \tau) \\
& +q_{x} A \sum_{n=-\infty}^{\infty} C_{2 n+2} \cos ((2 n+\beta) \tau) \\
& +i 2 q_{x} B \sum_{n=-\infty}^{\infty} C_{2 n} \cos (2 \tau) \sin ((2 n+\beta) \tau)
\end{aligned}
$$

where in the last step we just altered the indexing (allowed since the sum goes between $\pm \infty$ ). This allows us to easily match the cosine terms, yielding a recursion

\footnotetext{
${ }^{3}$ The product-to-sum relation is $\cos (\alpha) \cos (\beta)=\frac{1}{2}(\cos (\alpha+\beta)+\cos (\alpha-\beta))$.
} 
relation.

$$
-K_{2 n} C_{2 n}+C_{2 n-2}+C_{2 n+2}=0, \text { with } K_{2 n}=\frac{(2 n+\beta)^{2}}{q_{x}}
$$

This recursion relation allows us to calculate some useful relations. By setting $n=0$ in Eq. 3.20, we obtain an expression for $\beta$.

$$
K_{0}=\frac{\beta^{2}}{q_{x}}=\frac{C_{-2}+C_{2}}{C_{0}}
$$

Rearranging Eq. 3.20, we also find

$$
\frac{C_{2 n}}{C_{2 n+2}}=\frac{1}{K_{2 n}-\frac{C_{2 n-2}}{C_{2 n}}}
$$

and we can then plug Eq. 3.22 back into itself recursively, and obtain the continued fraction expression

$$
\frac{C_{2 n}}{C_{2 n+2}}=\frac{1}{K_{2 n}-\frac{1}{K_{2 n-2-\frac{1}{\ldots}}}}
$$

Similarly, we also get

$$
\frac{C_{2 n}}{C_{2 n-2}}=\frac{1}{K_{2 n}-\frac{1}{K_{2 n+2}-\frac{1}{\cdots}}}
$$

Plugging Eqs. 3.24 and 3.23 into Eq. 3.21 allow the calculation of $\beta$ to any order in $q_{x}$. Explicitly, we have

$$
\begin{aligned}
\beta^{2} & =q_{x}\left(\frac{C_{-2}}{C_{0}}+\frac{C_{2}}{C_{0}}\right) \\
& =q_{x}\left(\frac{1}{K_{-2}-\frac{1}{K_{-4}-\frac{1}{\cdots}}}+\frac{1}{K_{2}-\frac{1}{K_{4}-\frac{1}{\cdots}}}\right)
\end{aligned}
$$

To lowest order in $q_{x}$, we find

$$
\begin{aligned}
\beta^{2} & \approx q_{x}\left(\frac{1}{K_{-2}}+\frac{1}{K_{2}}\right) \\
& \approx q_{x}\left(\frac{q_{x}}{4}+\frac{q_{x}}{4}\right) \\
& =\frac{q_{x}^{2}}{2}
\end{aligned}
$$


and thus

$$
\beta \approx \frac{q_{x}}{\sqrt{2}}
$$

Given the above expressions for $\beta$ and the coefficients $C_{2 n}$, we can solve for the trajectory of the ion, $x(\tau)$. Assuming $q_{x} \ll 1$, we will take $C_{ \pm 4} \approx 0$. By assuming the initial condition $B=0$, from Eq. 3.17 we then obtain

$$
x(\tau) \approx A C_{0} \cos (\beta \tau)+A C_{-2} \cos ((\beta-2) \tau)+A C_{2} \cos ((\beta+2) \tau)
$$

Since $q_{x} \ll 1$, Eqs. 3.23 and 3.24 can be approximated as

$$
C_{0} \approx \frac{C_{ \pm 2}}{K_{0}+\frac{1}{K_{\mp 2}}}=\frac{C_{ \pm 2}}{\frac{\beta^{2}}{q_{x}}-\frac{1}{\frac{(\beta \mp 2)^{2}}{q_{x}}}} \approx \frac{C_{ \pm 2}}{\frac{q_{x}}{2}-\frac{q_{x}}{4}}=\frac{4 C_{ \pm 2}}{q_{x}}
$$

where in the second-to-last step, we used the approximation of Eq. 3.27 and $(\beta \mp 2)^{2} \approx$ 4. Plugging this value for $C_{ \pm 2}$ into Eq. 3.28 yields

$$
\begin{aligned}
x(\tau) & \approx A C_{0}\left[\cos (\beta \tau)+\frac{q_{x}}{4}(\cos ((\beta-2) \tau)+\cos ((\beta+2) \tau))\right] \\
& =A C_{0}\left[\cos (\beta \tau)+\frac{q_{x}}{2} \cos (\beta \tau) \cos (2 \tau)\right] \\
& =A C_{0} \cos (\beta \tau)\left[1+\frac{q_{x}}{2} \cos (2 \tau)\right]
\end{aligned}
$$

We can finally put this back in terms of $t$ by recalling our earlier substitutions

$$
\begin{aligned}
x(t) & \approx A C_{0} \cos \left(\frac{q_{x}}{2^{1 / 2}} \frac{\Omega_{T} t}{2}\right)\left[1+\frac{q_{x}}{2} \cos \left(\Omega_{T} t\right)\right] \\
& =A C_{0} \cos \left(\frac{e V_{0}}{2^{1 / 2} m \Omega_{T} R^{2}} t\right)\left[1+\frac{q_{x}}{2} \cos \left(\Omega_{T} t\right)\right] \\
& =A C_{0} \cos \left(\omega_{x} t\right)\left[1+\frac{q_{x}}{2} \cos \left(\Omega_{T} t\right)\right]
\end{aligned}
$$

where in the last step we have recalled the expression for the secular frequency given in Eq. 3.12. Thus, we have obtained the same secular oscillation that was derived by looking at the time-averaged force on the charged particle in Sec. 3.1.2. However, we now see superimposed on the secular motion a modulation at the driving 


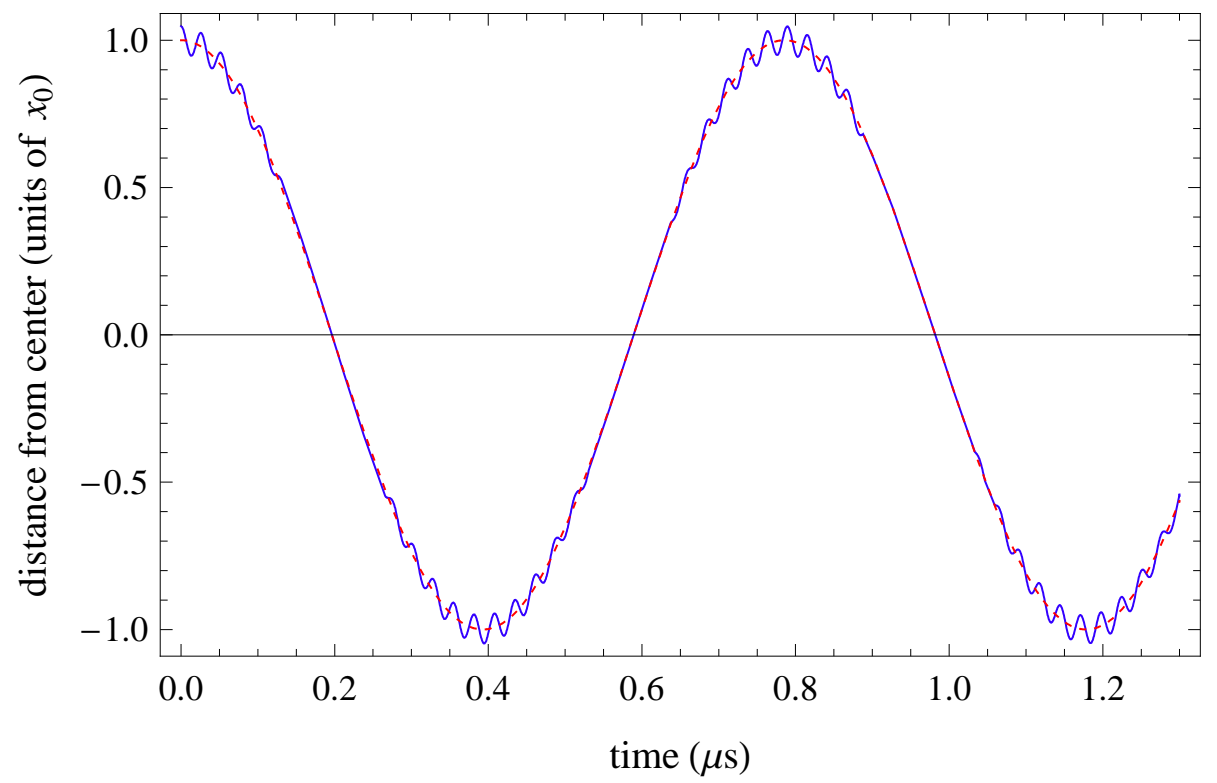

Figure 3.2: The motion of a trapped charged particle, as given by Eq. 3.31, with $R=0.46 \mathrm{~mm}$, $\Omega_{T} /(2 \pi)=38 \mathrm{MHz}, m=171 \mathrm{amu}$, and $V_{0}=1 \mathrm{kV}$. The slower, larger amplitude oscillation at $\omega_{x} /(2 \pi)=1.3 \mathrm{MHz}$ is the secular motion of particle, resulting from the time-averaged force of the inhomogenous electric field (red, dashed line). Superimposed on the secular motion is the micromotion that occurs at the drive frequency of trap $\left(\Omega_{T} /(2 \pi)=38 \mathrm{MHz}\right)$ with amplitude proportional to the excursion of the particle from the center of the quadrupole field (blue, solid line).

frequency of the trap, $\Omega_{T}$. The oscillation at $\Omega_{T}$ is known as the "micromotion," and has amplitude proportional to the distance from the center of the quadrupole field. Given that $q_{x} \ll 1$, the amplitude of the micromotion is typically much smaller than the secular motion. However, if a static offset field causes the secular motion to be centered a considerable distance from the center of quadrupole field, then it is possible for the amplitude of the micromotion to be much larger than the secular amplitude. In practice, static offset fields are carefully compensated to ensure the particle executes its secular motion about the center of the quadrupole field.

A representative picture of the motion of a particle confined by the quadrupole field of Eq. 3.9 is shown in Figure 3.2. In this case, we have taken $R=0.46 \mathrm{~mm}$, $\Omega_{T} /(2 \pi)=38 \mathrm{MHz}, m=171 \mathrm{amu}$, and $V_{0}=1 \mathrm{kV}$. By the equations above, we see this results in $\omega_{x} /(2 \pi)=1.3 \mathrm{MHz}$ and $q_{x}=0.1$. 


\subsection{Trap simulations}

The hyperbolic electrode structure presented in the previous section produces the ideal quadrupole potential for trapping charged particles. However, in practice we are often required to alter the structure of the trap electrodes to conform to other design parameters, such as optical access, multiple trapping zones, etc. Nevertheless, as long as we retain the overall symmetry presented in the previous section, then near the trap center the potential can be approximated by the hyperbolic potential given in Eq. 3.8 [41]. The effect of non-hyperbolic electrodes can be characterised by the addition of a geometric scaling factor $\eta_{s c}$ (generally of order unity), so that the modified potential near the center of the trap is approximately represented by [43]:

$$
\phi_{\text {nonhyp }}=\frac{\eta_{s c} V_{0}}{2} \cos \left(\Omega_{T} t\right)\left(1+\frac{x^{2}-y^{2}}{R^{2}}\right)
$$

Since $\eta_{s c}$ is just a constant factor, it carries through the rest of the equations derived in the previous section.

Before constructing an rf trap of arbitrary geometry, we usually perform a numerical simulation of the candidate ion trap to ensure the expected properties are consistent with the requirements of the experiment and the capabilities of the available equipment. An electrostatic simulation is justified by the fact that the wavelength of oscillation of the potential applied to the electrodes (order of meters) is typically much larger than the size of the trap $(\leq 1 \mathrm{~mm})$.

The basic procedure will be to first enter the trap structure design into the appropriate numerical simulation software. We will then run the electrostatic simulation with $1 \mathrm{~V}$ applied to one of the electrodes, while holding the rest at ground; this process is iterated for each electrode in the trap structure, thereby producing a set of "electrode basis functions" that describe the spatial properties of the potential 


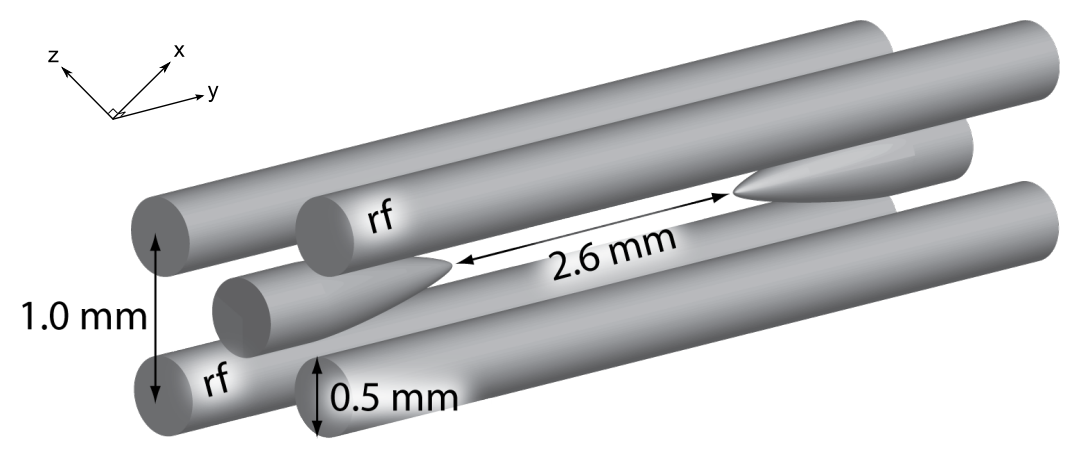

Figure 3.3: Four rod trap. The trap design shown here is the type used for all experiments presented in this work. Two of the four rods have radiofrequency (rf) potentials applied to create an oscillating quadrupole field for confinement in the $x, z$-plane. The other two rods are held at ground. Static potentials are applied to the needle electrodes to provide confinement along the $y$-axis of the trap.

produced by each electrode. The simulated potential of each electrode is sampled by a grid of points in the space, and exported to a file that can be used for further analysis. In a (possibly separate) analysis program, the potential from each electrode can be scaled to a voltage representing the proposed setup by simply multipling the electrode basis function by the appropriate value (e.g. if 70.2 volts is supposed to be applied, simply multiply the electrode basis function by 70.2 ). The total potential is then produced by simple summation of the separate electrode basis functions (for applied static potentials). The effect of the rf drive potential is modeled using the pseudopotential approximation of Eq. 3.7; so, the gradient of the electrode basis functions to which the rf drive is applied is used. Making the simulation step modular by generating the electrode basis functions allows for rapid reconfiguration of the applied voltages for testing a variety of experimental implementations. The fact that simple summation of the electrode basis functions produces the full potential is a simple consequence of the linearity of Laplace's equation [44].

In the following section, we demonstrate this method of trap simulation using the four-rod trap illustrated in Fig. 3.3 as an example. This is the design of the trap used in all of the experiments presented in the following chapters. 
3.2.1 CPO four-rod numerical simulation One of the most popular electrostatic simulation software packages in our lab (and others) is the 3D Charged Particle Optics program (CPO-3D) produced by CPO Ltd. ${ }^{4}$ In this section is a brief introduction to using this software to model candidate trap structures.

After starting the software package, open an example data file to edit by clicking

$$
\text { File } \Rightarrow \text { Open for running and databuilding }
$$

and selecting a sample file from the list. It doesn't matter which file you choose we're going to delete whatever electrodes are present in it and draw our own. Before doing so, though, be sure to save the file you've opened under a new name by clicking

$$
\text { File } \Rightarrow \text { Save As (data builder file) }
$$

and choosing a descriptive name for your project (e.g. FourRodTrap.dat).

Next, we want to design our trap structure by drawing the appropriate electrodes. Start this by clicking

$$
\text { Databuilder } \Rightarrow \text { Electrodes }
$$

so that the "Edit electrodes" window appears, as shown in Fig. 3.4(a). Before entering in our trap structure, delete all of the electrodes from the sample program by clicking the Delete button as many times as necessary to get rid of the original electrodes. We can then add our own electrodes by clicking the Add button. In the subsequent dialog box, choose the type/shape of the electrode (e.g. for the four-rod trap, we've selected "complete cylindrical surface"). The resulting window, shown in Fig. 3.4(b), allows us to enter in the parameters for this electrode. In the first box,

"Comment line": give the electrode a descriptive name (e.g. dc rod 1)

\footnotetext{
${ }^{4} \mathrm{~A}$ free evaluation version is available on the CPO website: http://www.electronoptics.com/.
} 
Define the dimensions of the electrode; for the case of the cylinder we have,

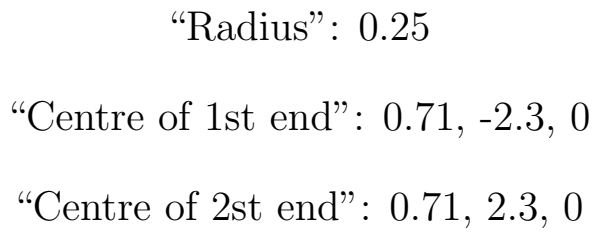

where the three numbers separated by commas refer to the separate boxes for the coordinates $x, y, z$. Choose the address of the voltages to be applied to this electrode,

"Addresses of two voltages that will be applied (usually the same)": 2, 2

Inputing two separate addresses allows us to create a voltage gradient across the electrode. However, for almost everything we do, these two addresses will be the same (e.g. 2, 2). Note that this entry is a voltage address, not the actual voltage being applied - we will define the voltages in a subsequent step. For now, we just need to be sure that electrodes that will require different voltages are assigned to separate voltage addresses. ${ }^{5}$ Finally, choose how to segment the electrode (for the purposes of the solving for the potential in the space around the electrode - the electrode is still considered one solid piece). In general, the larger the number of segments, the more precise the simulation will be, and the longer the computation time. As a side note, it is also generally not good practice to have small segments near large segments (these may occur when two electrodes are segmented differently, and placed near each other); CPO will usually produce a warning if this is the case. In addition, different versions of CPO have different restrictions on the total number of allowed segments. For simple electrodes far from the center of the trap (as in the present case) just a few segments will usually suffice. For example,

"Total nr subdivs, or subdivs along axis": 3

\footnotetext{
${ }^{5}$ Note: 0 is NOT a valid address - CPO will crash if you try to run it with this value.
} 


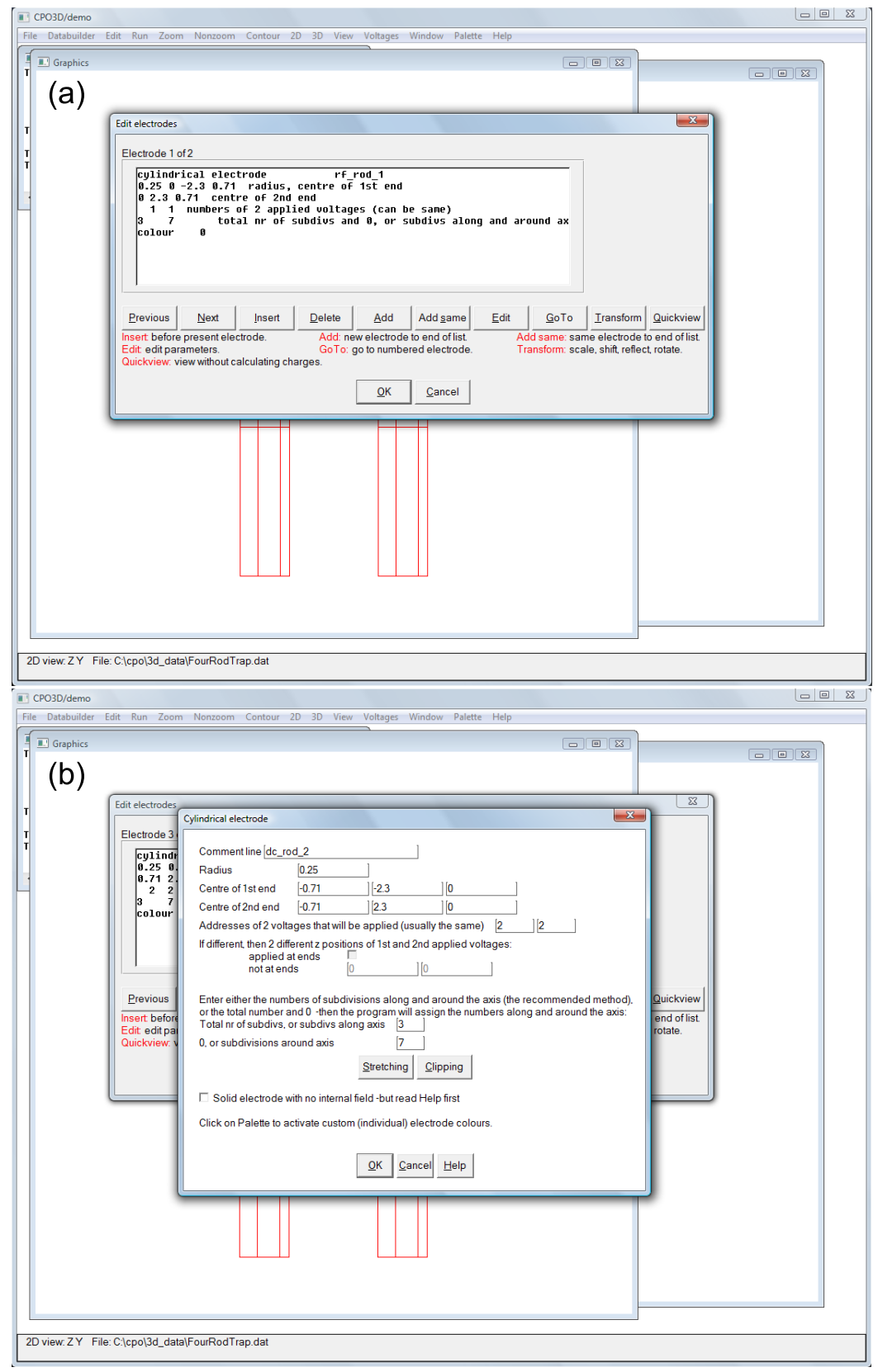

Figure 3.4: Adding electrodes in CPO. 
"0, or subdivisions around axis": 7

is what was used in the present simulation. Click OK to complete the description for this electrode, and then repeat the above steps until all electrodes have been added (if your structure has a good deal of symmetry, read below before adding all electrodes). Then click OK on the "Edit electrodes" window, and save your file (File $\Rightarrow$ Save (data builder file)).

The next step is to check the symmetries being applied. Access the symmetries options by hitting

$$
\text { Databuilder } \Rightarrow \text { Symmetries }
$$

The resulting window shows the symmetries you can choose to apply to your project (Fig. 3.5(a)). The time needed to design a trap structure can often be vastly reduced by choosing the appropriate symmetry options. In addition, CPO claims using symmetries improves the accuracy and reduces the computation time of the simulation. In the present case, though, we will not use any symmetry options, so we set all available options to "none", and click OK.

As stated previously, we are interested in creating a set of "electrode basis functions." The basis functions will be generated by iteratively assigning $1 \mathrm{~V}$ to each electrode, while holding the rest at ground. ${ }^{6}$ The simulation run then yields the spatial variance of the potential produced by that electrode. The full potential of the entire trap is obtained later offline, by multiplying each electrode basis function by the actual voltage to be applied and summing the potentials produced by each electrode (using the formula for the pseudopotential for electrodes with oscillating voltages). A simulation that is modular in this way allows us to model a variety of

\footnotetext{
${ }^{6}$ Actually, in each simulation run we may assign voltage to more than one electrode, if voltage will be applied symmetrically in the experiment: for instance, the rf electrodes.
} 


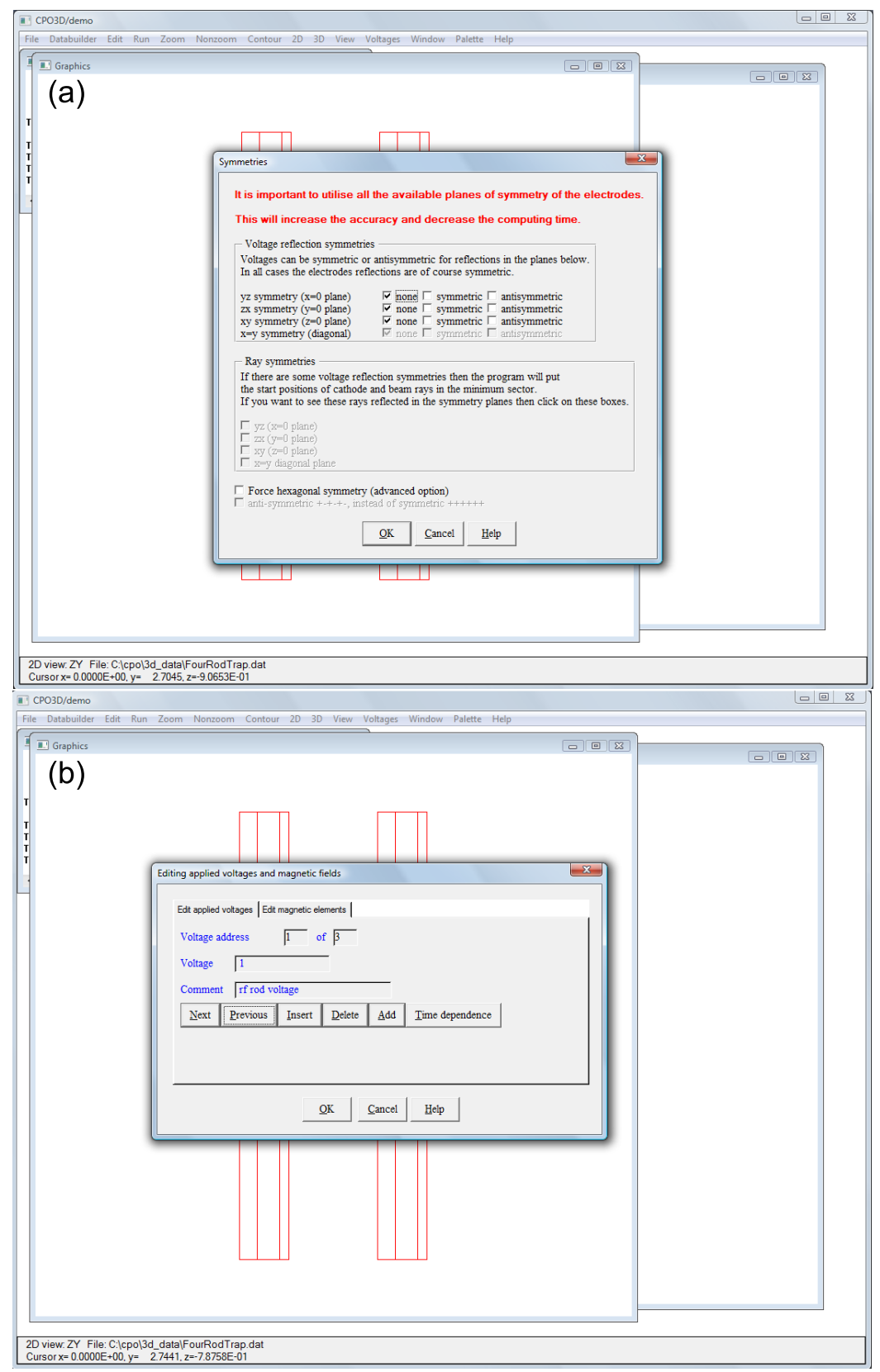

Figure 3.5: Adjusting symmetry and voltages in CPO. 
experimental setups with minimal simulation time.

Apply voltage to an electrode by going to

$$
\text { Databuilder } \Rightarrow \text { Voltages and magnetic fields }
$$

so that the "Editing applied voltages and magnetic fields" dialog box appears, as shown in Fig. 3.5(b). For each voltage address assigned to an electrode (recall what addresses you used when constructing your electrodes), input the desired applied voltage in "Voltage", and give it a descriptive name in the "Comment" box. For example, to model the rf electrodes in our four-rod trap, we make the assignments:

$$
\begin{gathered}
\text { "Voltage": } 1 \\
\text { "Comment": rf rod voltage }
\end{gathered}
$$

Add additional voltages by pressing the Add button, until you've created and assigned all of the voltage addresses used when you constructed your electrodes. Click OK to exit the window; then save your file.

We are now ready to run the simulation to create the basis function for this electrode. Run the simulation by hitting

$$
\text { Run } \Rightarrow \operatorname{All} 3 \text { views }
$$

If no error messages appear, we're in good shape! CPO is generally very fast; for the very simple four-rod trap design, the simulation takes less than 10 seconds. After the simulation has been run, a three-dimensional rendering of your electrode structure can be viewed by clicking 3D; our example four-rod trap is shown in Fig. 3.6(a). Use the buttons in the "3D view" window to change the perspective, and look at your creation from any angle.

There are only a limited number of analysis tools in CPO. The full analysis of the trap structure will be completed in Mathematica by importing all of the electrode 


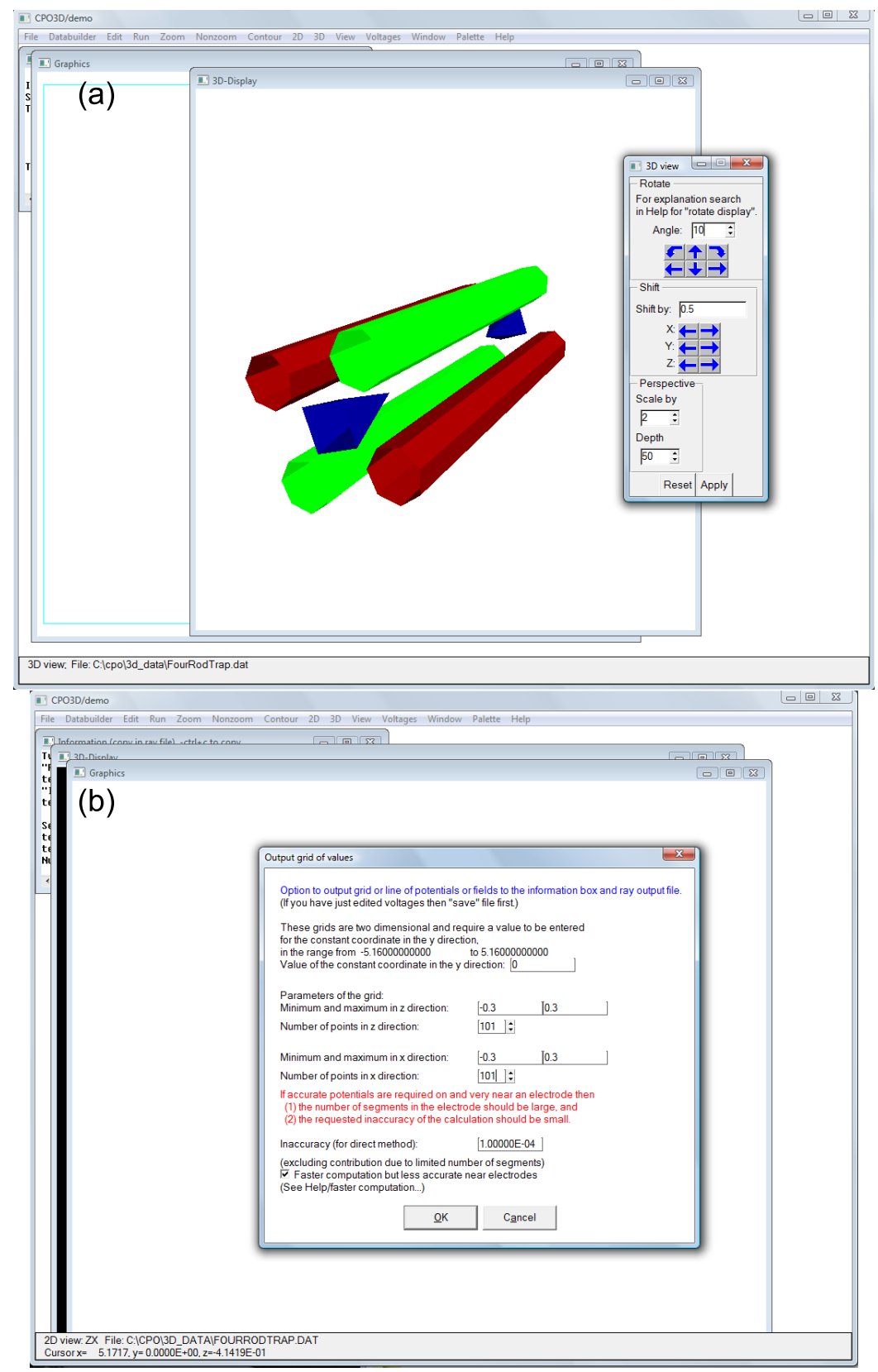

Figure 3.6: Views and exporting data in CPO. 
basis functions. At this point, then, we need to export an array of potential values calculated by CPO. Export a two-dimensional grid by first clicking 2D to return to a planar view of the electrode structure. Next, view the plane you want to export from by clicking (for example)

$$
\text { View } \Rightarrow \mathrm{ZX}
$$

In our four-rod trap example, we designed the rods of the trap to be perpendicular to the $x, z$-plane. Thus, in this plane we should see something resembling a quadrupole potential. Export a grid of points by first hitting

$$
\text { Contour } \Rightarrow \text { Potential } \Rightarrow \text { Grid }
$$

making the "Output grid of values" window appear, as seen in Fig. 3.6(b). In the available boxes, choose the point in the third dimension (for the $x, z$-plane, this would be the value for $y$ ), the range of values to be used in the two dimensions of the plane ( $x$ and $z$, in our case), the number of points to calculate in this range, and the accuracy CPO should attempt to achieve (the default value of $10^{-4}$ is probably fine). For the example of the four-rod trap, we choose:

"Value of the constant coordinate in the y direction": 0

"Minimum and maximum in the z direction": $-0.3,0.3$

"Number of points in z direction": 101

"Minimum and maximum in the $\mathrm{x}$ direction": $-0.3,0.3$

"Number of points in x direction": 101

"Inaccuracy (for direct method)": 1.00000E-04

and click OK to export the grid. If you're running the full version of CPO, the grid will output to a file in the $\mathrm{CPO}$ directory. On the other hand, if you're just using 
the demo version, the grid will only appear in the "Information" window, and it will be required to copy and paste the grid into a text document. ${ }^{7}$

Generate the remaining electrode basis functions by altering the applied voltages (Databuilder $\Rightarrow$ Voltages and magnetic fields), reruning the simulation (Run $\Rightarrow$ All 3 views), and exporting a grid of potential values (Contour $\Rightarrow$ Potential $\Rightarrow$ Grid) for each electrode.

3.2.2 Simulation analysis After completing the numerical simulation of the electrode basis functions in $\mathrm{CPO}$, we import the simulation data into a program with more advanced mathematical manipulation tools for analysis. In this case we chose to work with Mathematica, and a sample of the analysis code used is given in App. B. In the present section, we simply outline the general procedure pursued, and the results obtained.

We begin by importing the simulation data generated by CPO for each electrode basis function. The total effective potential of the trap is created by converting the potential of the rf electrode basis functions to the pseudopotential using Eq. 3.6, multiplying it by a scale factor representing the amplitude of the rf voltage applied, and adding this to the static potentials of the remaining electrode basis functions (each also multiplied by a scaling factor equal to the static voltage applied to each of these electrodes). The full potential is then given by

$$
\phi_{s i m}=\frac{e}{4 m \Omega_{T}^{2}} \nabla \sum_{j=1}^{N_{r f}}\left(V_{r f, j} \phi_{r f, j}\right)+\sum_{k=1}^{N_{d c}}\left(V_{d c, k} \phi_{d c, k}\right)
$$

where $N_{r f}$ and $N_{d c}$ are the number of rf and static electrodes, respectively; $V_{r f, j}$ is the rf voltage applied to the $j$ th rf electrode, with numerically calculated electrode basis function $\phi_{r f, j}$; and $V_{d c, k}$ is the static voltage applied to the $k$ th electrode, with

\footnotetext{
${ }^{7}$ Note: when selecting text to copy, start from the beginning of the grid; otherwise, the program crashes when Ctrl-C is pressed.
} 

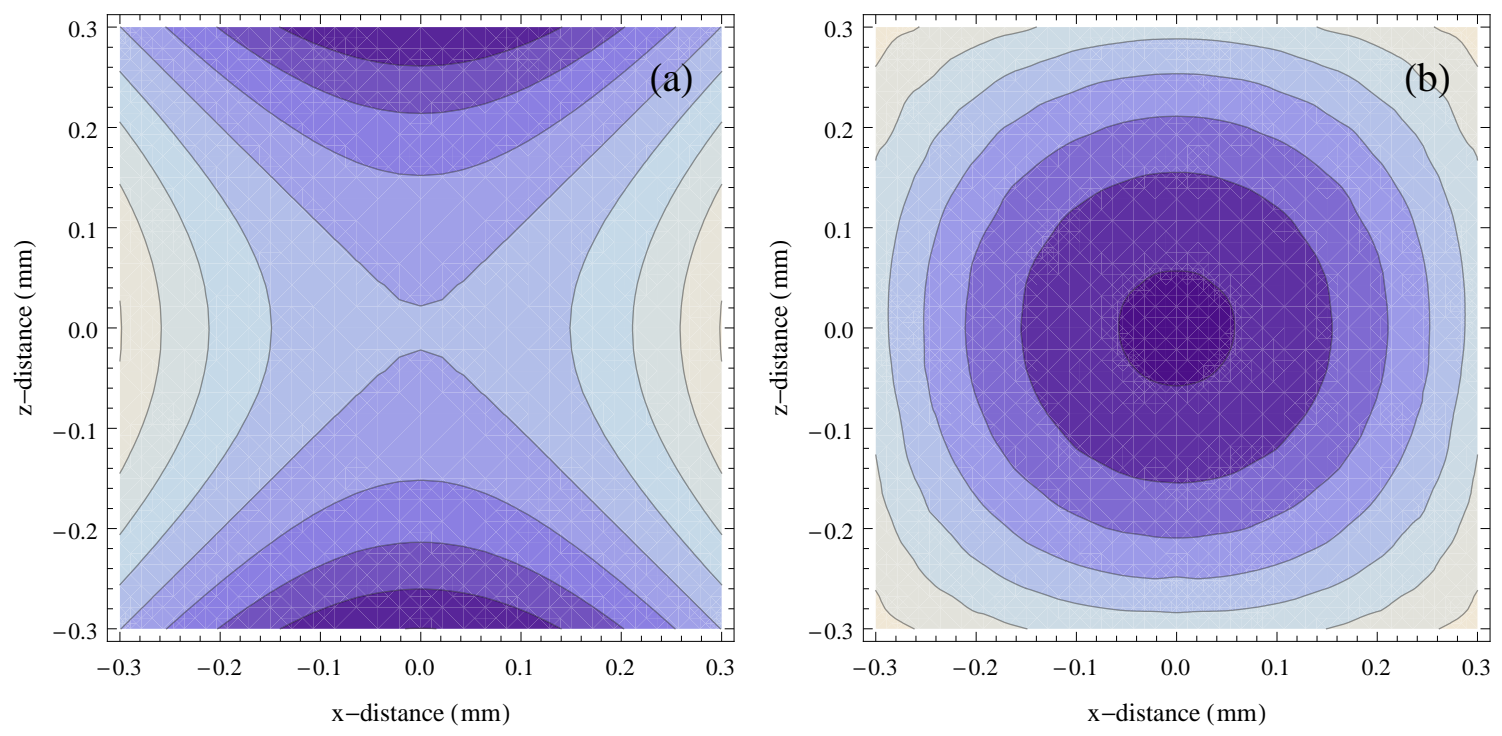

Figure 3.7: Numerical simulation of four-rod trap potentials. (a) The quadrupole potential produced by application of voltage to the two rf rods, while the other electrodes are held at ground (shown for the point of maximum amplitude in the oscillation). (b) The pseudopotential derived from the quadrupole potential of (a), using Eq. 3.6.

basis function $\phi_{d c, k}$. The simulated quadrupole potential for the four-rod trap is shown in Fig. 3.7(a), with the resulting pseudopotential illustrated in Fig. 3.7(b). Comparing Fig. 3.7(a) with the ideal quadrupole potential of Fig. 3.1, we see that near the center of the trap the four rods produce a potential nearly identical to that generated by ideal hyperbolic electrodes.

The motion of a charged particle in a trap can be viewed as a three-dimensional, uncoupled harmonic oscillator along the principal axes of the trapping potential. In other words, motion of the particle along one of the principal axes of a trap is independent of the other two principal axes. Therefore, the principal axes of the trap present a natural coordinate system for the electrode structure. In addition, knowledge of the orientation of the principal axes is vital for efficient laser cooling; if the incident light is perpendicular to one of the principal axes, the particle will not be cooled along that direction. While in simple trap structures such as our 
four-rod trap, the orientation of the principal axes is clear from the symmetry of the electrodes, in general we determine the principal axes of the trap by use of the Hessian matrix of the potential, which in two-dimensions is [45]: ${ }^{8}$

$$
H\left(\phi_{\text {sim }}\left(x_{0}, z_{0}\right)\right)=\left(\begin{array}{cc}
\frac{\partial^{2} \phi_{\text {sim }}}{\partial x^{2}}\left(x_{0}, z_{0}\right) & \frac{\partial^{2} \phi_{\text {sim }}}{\partial x \partial z}\left(x_{0}, z_{0}\right) \\
\frac{\partial^{2} \phi_{\text {sim }}}{\partial z \partial x}\left(x_{0}, z_{0}\right) & \frac{\partial^{2} \phi_{\text {sim }}}{\partial z^{2}}\left(x_{0}, z_{0}\right)
\end{array}\right)
$$

Here $x_{0}$ and $z_{0}$ are the point where the Hessian matrix is evaluated, which in our case would be the center of the trap. The eigenvalues of this matrix are related to the angle by which the principal axes are rotated with respect to the coordinate axes (used in the partial derivatives). In other words, the Hessian matrix seeks out the directions of greatest and least curvature, which are precisely the principal axes.

After determining the principal axes of the trap, we fit the potential along this axis to a parabola (other polynomial terms may be included in the fit, but if the trap produces the potential we expect, the quadratic term of the fit should dominate). The quadratic coefficient of the fit, $A_{\text {prin }}$, allows the determination of the secular frequency along this axis.

$$
\omega_{\text {prin }}=\sqrt{\frac{2 e A_{\text {prin }}}{m}}
$$

where the factor of $e$ was used to convert the electric potential to energy.

The four-rod trap can now be evaluated for specific parameters, and compared to the ideal hyperbolic case to determine the geometric scale factor, $\eta_{s c}$. Taking $V_{r f}=1 \mathrm{kV}, V_{d c}=80 \mathrm{~V}, m=171 \mathrm{amu}, R=0.5 \sqrt{2}-0.25=0.46 \mathrm{~mm}$, and $\Omega_{T}=38$ $\mathrm{MHz}$, we calculate a secular frequency $\omega_{x, \text { sim }} /(2 \pi)=1.17 \mathrm{MHz}$ for the four-rod trap. Plugging these same parameters into Eq. 3.12 for the ideal hyperbolic trap, we find $\omega_{x, h y p} /(2 \pi)=1.26 \mathrm{MHz}$. Thus, the geometric scale factor is $\eta_{s c}=\omega_{x, \text { sim }} / \omega_{x, h y p}=$ 0.93. The total depth of the trap can also be determined by the simulation from the

\footnotetext{
${ }^{8}$ In a linear trap, the third principal axis is always clear from structure of the trap. In Fig. 3.3, it is parallel to the rods of the trap (along the line defined by the needle electrodes).
} 
depth of the pseudopotential-well, and for these parameters we find the trap depth to be approximately $10 \mathrm{eV}$. This trap depth corresponds to a temperture of more than $10^{5} \mathrm{~K}$, reiterating the fact that the rf trap provides excellent confinement of charged particles.

\subsection{Vacuum chamber and trap construction}

The experiments presented in the following chapters were performed on atomic ions trapped in an ultra-high vacuum (UHV) environment, to ensure isolation of the atoms and long trapping lifetimes. At a pressure of about $10^{-11}$ torr, we expect an average of 2 collisions with background gas every hour. Given the depth of the trap, generally such collisions will not eject the ion from the trap. Moreover, since each iteration of the experiment usually lasts between $1 \mu \mathrm{s}$ and $2 \mathrm{~ms}$, collsions should not noticeably alter the experiment. On the other hand, inelastic collisions that result in chemical reactions could limit the trapping lifetime. ${ }^{9}$ The lifetime of an atom in the trap could also be limited by defective trap components, or be precluded altogether if the atomic source does not produce the necessary atoms. Hence, proper construction and assembly of the vacuum chamber, atomic source ovens, and trap are essential to the atomic physics experiments.

3.3.1 Chamber assembly The vacuum chamber used in the experiments of the subsequent chapters is shown schematically in Fig. 3.8. Standard ConFlat (CF) vacuum components were used to construct the chamber. As the vacuum seal of a CF component is all metal, consisting of stainless steel edges compressing a copper gasket, they can be used at high temperatures. Given that baking the chamber (outlined in Sec. 3.3.4) is vital to achieving UHV, CF components are a logical

\footnotetext{
${ }^{9}$ Indeed, the formation of molecules is a known loss mechanism in ion traps. Fortunately, the $\mathrm{YbH}^{+}$molecule actually has a photodissociation line near the wavelength used to Doppler cool the atoms [46]. We have seen that application of strong laser light near $369.5 \mathrm{~nm}$ will renew the fluorescence of an ion that had gone dark.
} 
choice for the chamber construction. Before assembling the chamber, each all-metal component is wrapped in aluminum foil and pre-baked at a temperature of 250 ${ }^{\circ} \mathrm{C}$ for about a week. The pre-bake of these components establishes a chromiumoxide layer on the surface of the steel that reduces outgassing of hydrogen [47]. The components are then thoroughly cleaned in an ultrasonic acetone bath, and assembled into their final configuration. During assembly, strict care is taken to prevent organic compounds (body oils, hair, etc.) from contaminating the chamber. Specialty components include: the vacuum ion gauge; the $20 \mathrm{~L} / \mathrm{s}$ ion pump; the titanium sublimation (Ti-sub) pump; two vacuum feedthroughs, each with four pins, for electrical connections to the ion trap and atomic source ovens; six small windows and two large, recessed windows (viewports) for optical access; and, of course, the ion trap.

3.3.2 Trap assembly The four-rod ion trap depicted schematically in Fig. 3.3 is held inside the spherical octagon ${ }^{10}$ by an invar mounting bracket, shown in Fig. 3.9(a) and visible in the image of Fig. 3.9(c). The electrodes of the trap are tungsten, with the material chosen because it is nonmagnetic and has high tensile strength, high melting point, and low vapor pressure [48]. Each of the four rods has a diameter of $0.25 \mathrm{~mm}$ and is approximately $40 \mathrm{~mm}$ in length (greater than the length of the central cage of the mounting bracket). The needles are simply short tungsten rods that have been sharpened and then electropolished using sodium hydroxide to obtain a sharp point. ${ }^{11}$ The electrodes are isolated from the mounting bracket and the rest of the chamber by two alumina spacers fit into the holes on either side of the mounting bracket. Each alumina spacer has five holes, four of which are arranged symmetrically about the perimeter, and the fifth directly through the center. The four rods of the trap

\footnotetext{
${ }^{10}$ Spherical octagon is from Kimball Physics, part number MCF450-SO20008.

${ }^{11} \mathrm{~A}$ helpful list of eletropolishing recipes is available at www.fischione.com/ product_support/ model_110_application_notes.asp.
} 


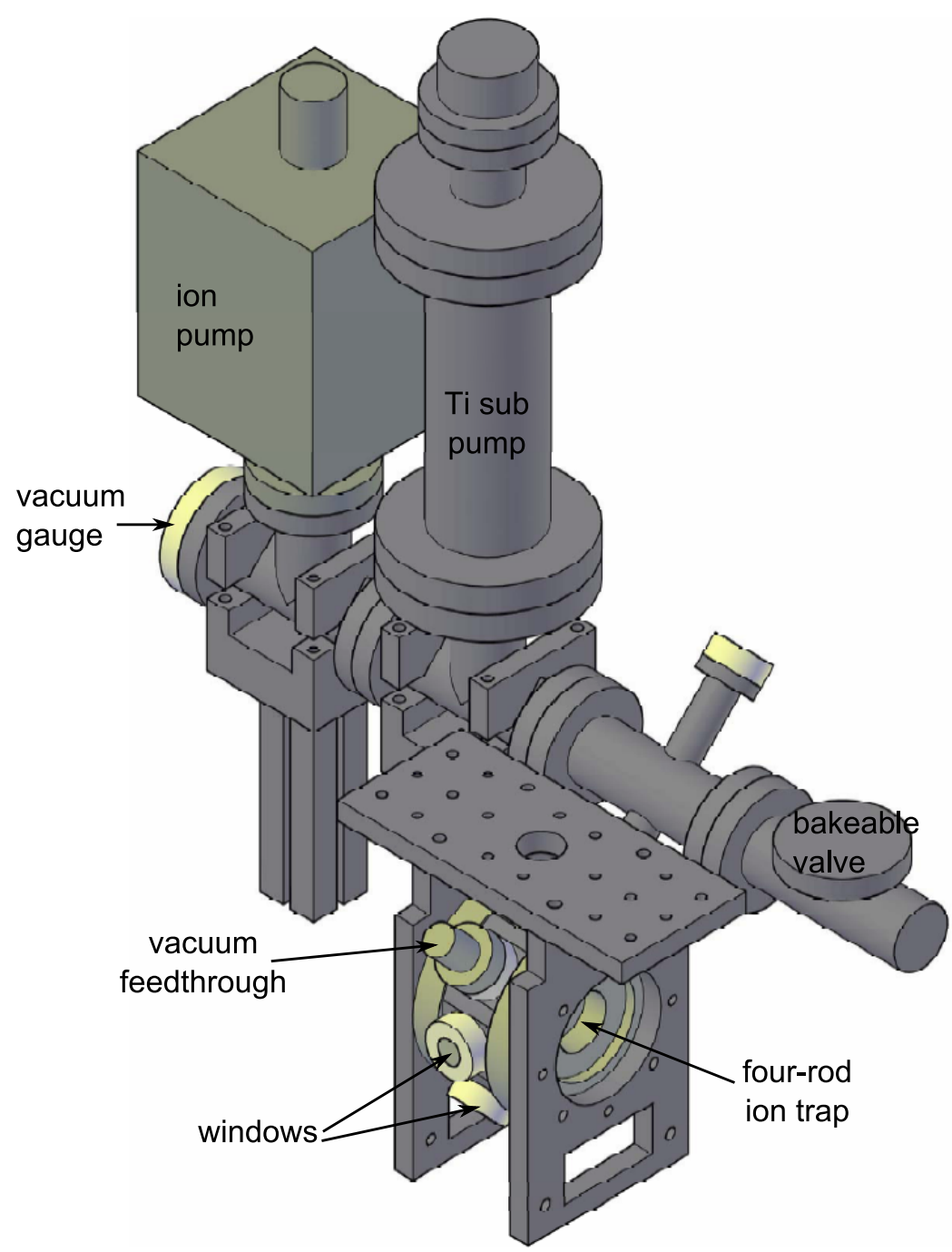

Figure 3.8: Schematic of vacuum chamber employed in these experiments. The chamber was constructed using standard ConFlat $(\mathrm{CF})$ vacuum components. Labeled in the figure are: the vacuum ion-gauge, used to determine the pressure in the chamber; the $20 \mathrm{~L} / \mathrm{s}$ ion pump, which (after the bake) runs continuously to keep the chamber at ultra-high vacuum $\left(\approx 10^{-11}\right.$ torr); the titanium sublimation pump (Ti sub pump), which is run once every few months; the bakeable valve that was the internal chamber's last link to the external environment; one of two vacuum feedthroughs that allow electrical connection to the ion trap and atomic source ovens; two of the eight windows (six small, two large) that allow optical access; and finally, the ion trap (not actually visible) which resides inside the spherical octagon. 


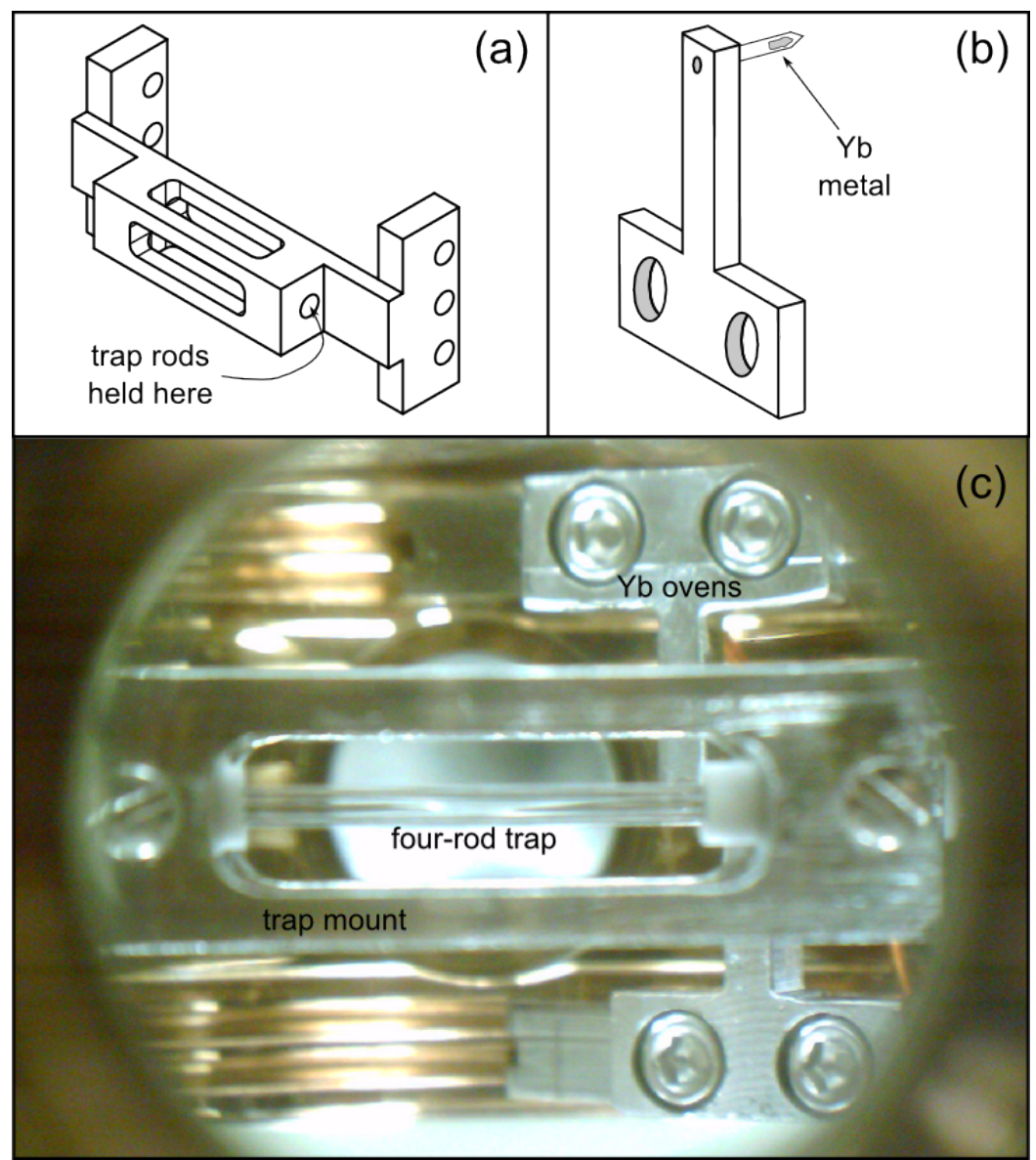

Figure 3.9: Ion trap in mount and $\mathrm{Yb}$ ovens. (a) Schematic of the mount for the ion trap. (b) Schematic of the $\mathrm{Yb}$ ovens. (c) Image of the ion trap in the mount, with the $\mathrm{Yb}$ ovens visible in the background, during construction of the vacuum chamber.

are fit through the four outer holes of each spacer, while each needle is supported by being fit through the center hole on one of the spacers and arranged such that the tips are opposed to each other. A schematic of the final trap structure is shown in Fig. 3.3, and one of the actual traps is shown in the image of Fig. 3.9(c).

3.3.3 Atomic ovens The atomic source ovens are fabricated from a small piece of aluminum and a small stainless steel tube, as illustrated schematically in Fig. 3.9(b). The aluminum block is used for mechanical mounting to the chamber, and is machined into the shape of a "T" to minimize its effect as a heat sink on the stainless steel tube. The end of this tube is crimped shut so that the tube can be packed 


\begin{tabular}{|c||c|c|}
\hline \multicolumn{1}{|c||}{} & \multicolumn{2}{c|}{ Time to Spot (min) } \\
\hline Current (amps) & Natural Oven & 171 Oven \\
\hline \hline 6.0 & n.a. & 2.0 \\
5.0 & 8.0 & 7.0 \\
4.5 & 20.0 & 16.5 \\
4.0 & 37.0 & 35.0 \\
3.5 & $>60$ & $>45$ \\
\hline
\end{tabular}

Table 3.1: East trap oven tests. The first column shows the operating current (amps). The second and third columns show the time elapsed before a visible spot is seen from the ovens containing ytterbium metal of natural abundance and isotopically enriched, respectively. At an operating current of 3.5 amps, a spot was not observed from either oven; the time given at this current is thus a lower bound.

partially (about a third full) with ytterbium metal. The open end of the tube is situated in the chamber to aim through the center of the trap at one of the walls of the chamber (not aimed at a window). An atomic "spray" is generated by passing a current through the tube, resistively heating it. The thermal properties of each such oven will depend critically on the size of the aluminum mount, the length and thickness of the stainless steel tube, etc. Since too much atomic flux can have adverse effects on the chamber, each atomic oven is charaterized before being mounted in the vacuum chamber. ${ }^{12}$ The operation parameters of each oven is determined by mounting the ovens temporarily in a bell jar that is pumped down to a pressure of about $10^{-5}$ torr. The ovens are run with a range of different currents, with the resulting atomic flux at each current level being (roughly) characterized by the time it takes to produce a visible spot of ytterbium metal on the glass of the bell jar. An example of the result of these oven tests is given in Table 3.1.

3.3.4 Vacuum chamber bake One of the most important procedures for attaining UHV is the bake of the fully assembled vacuum chamber. The main purpose of the bake is to remove water trapped in the stainless steel, as hydrogen is one of the primary contributors to the pressure in UHV [47]. While the bake of a vacuum

\footnotetext{
${ }^{12}$ Ytterbium, in particular, sticks to almost everything. Thus, if the ovens are run too hard, you run the risk making your windows opaque.
} 


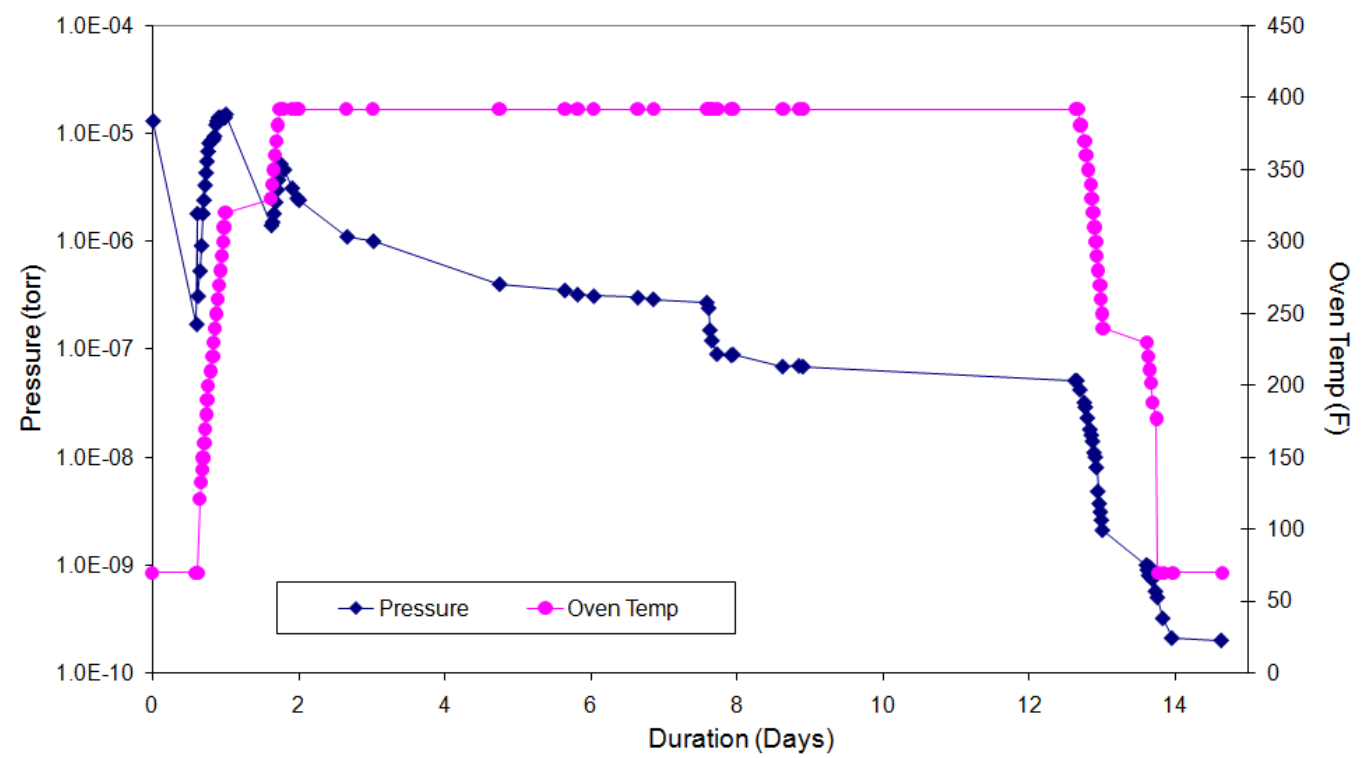

Figure 3.10: East trap bake, showing pressure (torr) and temperature $\left({ }^{\circ} \mathrm{F}\right)$ versus time (days). The pressures obtained during/after running the Ti-sub pump are not included in this plot.

chamber is often done using heater tape and fiberglass insulation, here we utilize a large industrial oven. The basic procedure is: (1) evacuate the chamber to about $10^{-6}$ torr with a roughing pump; (2) degas various components (ion gauge, Ti-sub pump, ovens) by running current through them for a short period of time; (3) close off the roughing pump, and open up to the large (500 L/s) ion pump; (4) turn on the oven, and slowly ramp (about $10^{\circ} \mathrm{F}$ every twenty minutes) the temperature up to $392{ }^{\circ} \mathrm{F}\left(200{ }^{\circ} \mathrm{C}\right)$; (5) bake for a few days, then switch on the small internal ion pump, closing off the large ion pump by hand-tightening the bakeable valve; (6) after baking a few more days, slowly ramp the temperature back to room temperature; (7) close the bakeable valve with the proper torque; (8) evacuate the space behind the bakeable valve with a pinch-off valve; (9) run the Ti-sub pump. A more detailed bake schedule for the East trap is given in Table 3.2, and the pressure as a function of time is shown in Fig. 3.10.

3.3.5 Trap operation Once the vacuum chamber is fully assembled and the bake is complete, it is ready for operation. One more trick in the operation of the trap is 


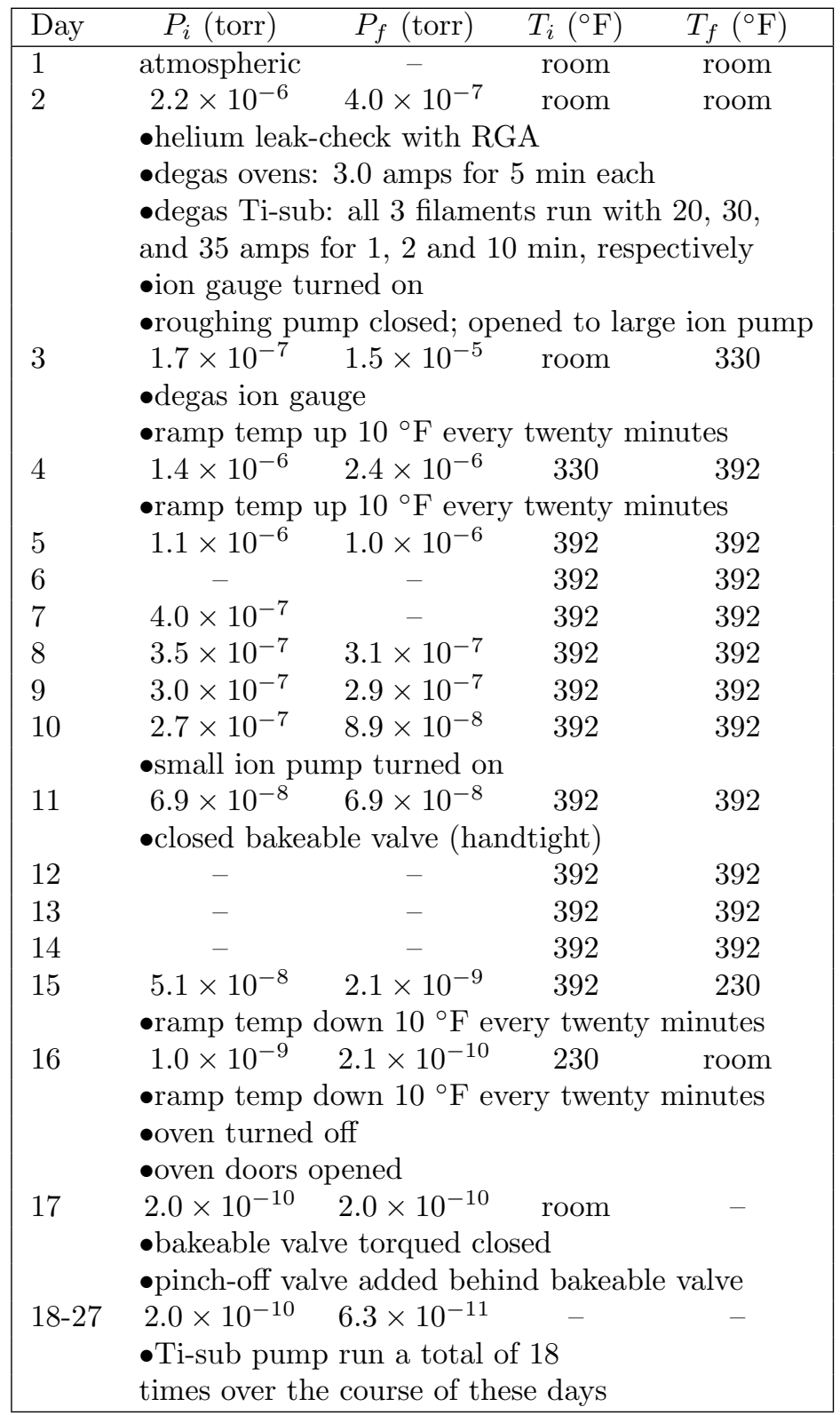

Table 3.2: East trap bake schedule. 
the application of the oscillating voltages that create the harmonic pseudopotential. Given that both the depth of the trap and the secular frequency of motion of a trapped particle are proportional to the amplitude of the applied rf voltage, it is usually desirable to have high rf voltages. One way of achieving this high voltage is by using a resonant circuit. Of course, the wavelength of a $35 \mathrm{MHz}$ signal is 8.6 meters, so to keep the size of the resonator reasonable, we use use the quarterwave helical design shown schematically in Fig. 3.11. The relations between design parameters for this type of helical resonator is found in Refs. [49, 50]. The helical resonator employed on one of our traps (east trap) is composed of a 3.75 inch long copper shield (4.5 inches including endcaps) 2.5 inches in diameter, and two inner 11 AWG copper helical coils $65 \mathrm{~mm}$ in length with 8 turns. ${ }^{13}$ The helical coil is held in place by three thin polystyrene spacers (cut from a CD case); polystyrene was chosen because of its low loss-tangent, but other, less brittle materials such as teflon also work well. With this design, we achieve a resonant frequency of $35 \mathrm{MHz}$ and loaded quality factor $Q=270$ when the resonator is connected to the ion trap. ${ }^{14}$ The voltage out of the resonator is given by $V_{r f}=\epsilon \sqrt{P_{r f} Q}$, where $P_{r f}$ is the input rf power, and $\epsilon$ is a geometric factor of about 20. Thus, by applying $8 \mathrm{~W}$ of rf at 35 $\mathrm{MHz}$, we expect the amplitude of the oscillating voltage on the trap electrodes to be $0.9 \mathrm{kV} .{ }^{15}$ The static voltages $(80 \mathrm{~V})$ for the needle electrodes are passed through a standard $\pi$-network filter to shunt any capacitively-coupled rf voltage.

The procedure for trapping and cooling ytterbium ions is presented in Chap. IV.

The atomic source oven of natural abundance is typically run for 30 seconds at a

\footnotetext{
${ }^{13}$ This two coil, or "bifilar," helical resonator is used so as to apply th same rf voltage to both rf rod electrodes (a capacitor connects the ends of each coil to ensure the phases are matched), but also to allow application of independent static bias voltages.

${ }^{14}$ Before connecting the resonator the trap the resonance frequency was $55 \mathrm{MHz}$ with a $Q=520$.

${ }^{15}$ The coupling efficiency, determined by measuring the power reflected by the resonator, was $99.975 \%$ (reflection $-36 \mathrm{~dB})$.
} 


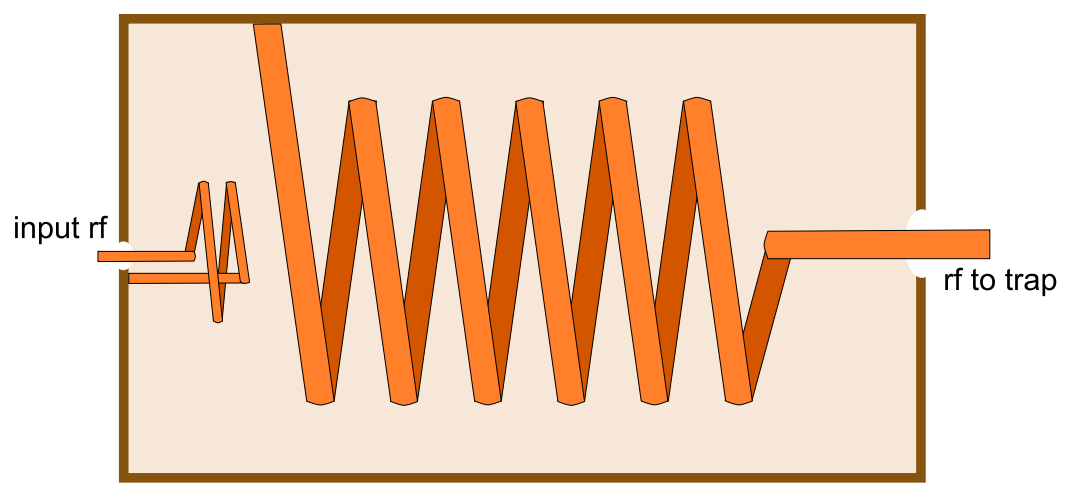

Figure 3.11: Simple diagram of the type of helical resonator that can be use to apply high voltage rf to the ion trap. The quarter-wave resonator consists of a copper helical coil inside a cylindrical copper shield. The small loop coil on the left is used to inductively couple the rf signal into the helical resonator. Note: for simplicity, shown is a single coil ("monofilar") resonator, not the two coil ("bifilar") resonator actually used in the experiment.

current of 2.75 amps to obtain a single ion, though this number depends on the alignment, power, and frequency of the photoionization beam. A single ion has been trapped for over 3 weeks without laser cooling, and we have observed retention times of over 3 months when the ion is periodically laser-cooled. The excellent trapping lifetimes of atomic ions are extremely beneficial for atomic physics experiments requiring long integration times, such as those presented in the following chapters. 


\section{CHAPTER IV}

\section{Ytterbium Ions}

\footnotetext{
"There is a theory which states that if ever anyone discovers exactly what the Universe is for and why it is here, it will instantly disappear and be replaced by something even more bizarre and inexplicable.

There is another which states that this has already happened."

-Douglas Adams, The Restaurant at the End of the Universe
}

Trapped atomic ions have long been recognized as a promising implementation of quantum bits (qubits) for quantum information processing [51, 41], due in part to long trapping lifetimes, long coherence times of particular internal electronic states, and the exquisite control attained over both the internal and external degress of freedom. The hydrogen-like ions that have been directly cooled and manipulated for applications in quantum information include $\mathrm{Ba}^{+}[52,53], \mathrm{Be}^{+}[54], \mathrm{Ca}^{+}[55,56,57]$, $\mathrm{Cd}^{+}[58], \mathrm{Mg}^{+}[59], \mathrm{Sr}^{+}[25]$, and $\mathrm{Yb}^{+}[60,61]$. In Table 4.1 various properties of these atomic ions are compared. 


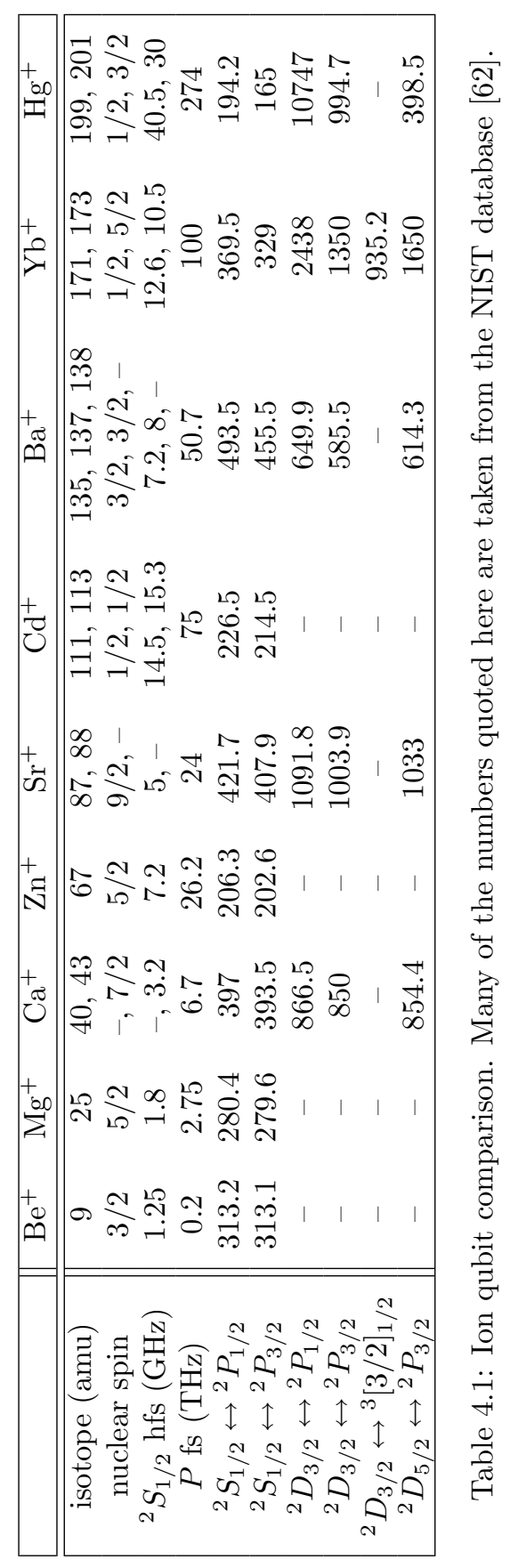


The ytterbium ion $\left(\mathrm{Yb}^{+}\right)$is especially attractive because the strong ${ }^{2} S_{1 / 2} \leftrightarrow{ }^{2} P_{1 / 2}$ electronic transition near $369.53 \mathrm{~nm}$ is suitable for use with optical fibers, making schemes that require the coupling of atomic (hyperfine) qubits to photonic (optical) qubits feasible $[63,28,64,13,65,14,66,67,68,15,69,70,71,11,72]$. Moreover, the large fine structure splitting of $\mathrm{Yb}^{+}$makes it amenable to fast manipulation with broadband laser pulses $[73,74,75,76]$. Finally, the spin-1/2 nucleus of ${ }^{171} \mathrm{Yb}^{+}$allows for simple, fast, and efficient preparation and detection of the ground state hyperfine levels.

\subsection{Photoionization}

Ytterbium ions $\left(\mathrm{Yb}^{+}\right)$are loaded into the trap by photoionization of neutral Yb. A thermal, directional spray of $\mathrm{Yb}$ atoms is produced by resistively heating one of the atomic source ovens (Sec. 3.3.3). A continuous-wave (cw) diode laser that provides approximately $5 \mathrm{~mW}$ of light at $398.91 \mathrm{~nm}$ is tuned to the ${ }^{1} S_{0} \leftrightarrow{ }^{1} P_{1}$ transition of neutral $\mathrm{Yb}$ and is focused through the center of the trap with a waist of approximately $50 \mu \mathrm{m}$. A second beam near $369.53 \mathrm{~nm}$ is generated by frequency-doubling the light produced by a cw amplified diode laser near $739.05 \mathrm{~nm}$. Approximately $375 \mathrm{~mW}$ of $739.05 \mathrm{~nm}$ light is sent to a resonant cavity containing a critically phase-matched LBO crystal, producing more than $14 \mathrm{~mW}$ at $369.53 \mathrm{~nm} .{ }^{1}$ During photoionization, about $2 \mathrm{~mW}$ of this light at $369.53 \mathrm{~nm}$ is aligned counter-propagating to the beam at $398.91 \mathrm{~nm}$, and is focused through the trap with a waist of about $30 \mu \mathrm{m}$. Neutral $\mathrm{Yb}$ atoms passing through these beams are photoionized by way of a resonantly assisted, dichroic, two-photon transition [60, 61]: the $398.91 \mathrm{~nm}$ light excites Yb atoms from the ${ }^{1} S_{0}$ to the ${ }^{1} P_{1}$ level, from which the $369.53 \mathrm{~nm}$ light can promote the electron to the continuum (Fig. 4.1). The atomic source ovens are aligned such that the

\footnotetext{
${ }^{1}$ The resonant cavity used for second harmonic generation is a commercial Spectra-Physics WaveTrain.
} 


\begin{tabular}{|c|ccccc|}
\hline & \multicolumn{5}{|c|}{ isotope mass (amu) } \\
& 170 & 171 & 172 & 174 & 176 \\
\hline Yb: & & & & & \\
${ }^{1} S_{0} \leftrightarrow{ }^{1} P_{1}$ & - & 398.9118 & 398.9116 & 398.9113 & 398.9111 \\
$\mathrm{Yb}^{+}:$ & & & & & \\
${ }^{2} S_{1 / 2} \leftrightarrow{ }^{2} P_{1 / 2}$ & 369.5237 & 369.5263 & 369.5245 & 369.5252 & 369.5256 \\
${ }^{2} D_{3 / 2} \leftrightarrow{ }^{3}[3 / 2]_{1 / 2}$ & 935.1982 & 935.1878 & 935.1874 & 935.1799 & 935.1724 \\
${ }^{2} F_{7 / 2} \leftrightarrow{ }^{1}[5 / 2]_{5 / 2}$ & - & 638.6101 & - & 638.6185 & - \\
& & 638.6151 & & & \\
${ }^{2} F_{7 / 2} \leftrightarrow{ }^{3}[5 / 2]_{3 / 2}$ & - & - & - & 864.8430 & - \\
\hline
\end{tabular}

Table 4.2: Ytterbium transitions by isotope, as measured by our wavemeter; determination of which isotope these fluorescence signals correspond to was made by comparison of the relative. All wavelengths shown are in nm. For the 171 isotope: the ${ }^{2} S_{1 / 2} \leftrightarrow{ }^{2} P_{1 / 2}$ given is for ${ }^{2} S_{1 / 2}|F=1\rangle \leftrightarrow{ }^{2} P_{1 / 2}|F=0\rangle ;$ the ${ }^{2} D_{3 / 2} \leftrightarrow{ }^{3}[3 / 2]_{1 / 2}$ given is for ${ }^{2} D_{3 / 2}|F=1\rangle \leftrightarrow$ ${ }^{3}[3 / 2]_{1 / 2}|F=0\rangle$; and the two values for ${ }^{2} F_{7 / 2} \leftrightarrow^{1}[5 / 2]_{5 / 2}$ are for the ${ }^{2} F_{7 / 2}|F=3\rangle \leftrightarrow$ ${ }^{1}[5 / 2]_{5 / 2}|F=2\rangle,{ }^{2} F_{7 / 2}|F=4\rangle \leftrightarrow{ }^{1}[5 / 2]_{5 / 2}|F=3\rangle$ transitions [81]. We have not attempted to observe the 168 (natural abundance is only 0.0013 [62]) or 173 (requires additional frequency sources to depopulate additional hyperfine levels) isotopes.

propagation direction of the neutral $\mathrm{Yb}$ atoms is approximately perpendicular to the $398.91 \mathrm{~nm}$ beam to minimize Doppler shifts and allow for isotopically selective loading (Table 4.2).

\subsection{Doppler cooling}

After photoionization of a neutral atom in the trap, the confined $\mathrm{Yb}^{+}$atom is Doppler-cooled by the light at $369.53 \mathrm{~nm}$, which is slightly red-detuned of the ${ }^{2} S_{1 / 2} \leftrightarrow$ ${ }^{2} P_{1 / 2}$ transition depicted in Fig. 4.2. Doppler cooling relies on the frequency detuning dependance of the photon scattering rate of the atom [82]:

$$
\Gamma_{s c}=\frac{\frac{I}{I_{s a t}} \frac{\Gamma}{2}}{1+\frac{I}{I_{s a t}}+\frac{4 \Delta_{l}^{2}}{\Gamma^{2}}}
$$

In this equation, $I$ is the intensity of the incident light; $\Gamma=1 / \tau$ is the spontaneous emission rate of the transition, with $\tau$ the natural lifetime of the excited state, and is related to the natural linewidth $\gamma=\Gamma / 2 \pi$; and $\Delta_{l}$ is the detuning of the incident light from the transition resonant frequency. We have also used the saturation intensity $I_{\text {sat }}$, defined as the intensity necessary to create equal population in the lower and 


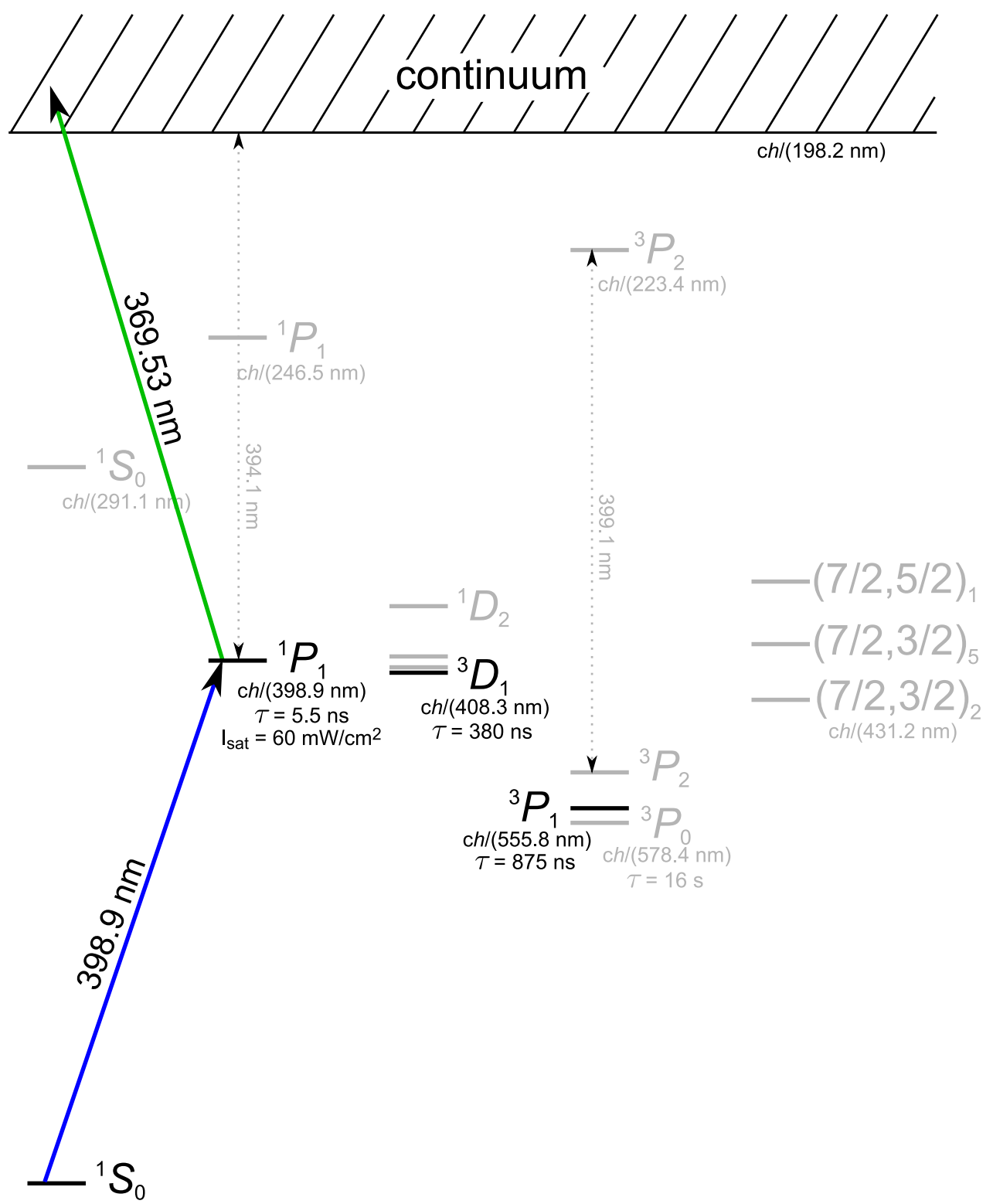

Figure 4.1: Partial level diagram of the neutral $\mathrm{Yb}$ atom (to scale). Level energies are given as $E=c h / \lambda$, and are quoted from Ref. [62]. The transitions shown with solid lines are driven by laser sources in the experiment to achieve resonant-assisted photoionization. The lifetime and saturation intensity of the ${ }^{1} P_{1}$ level is from Ref. [77]; lifetimes of the ${ }^{3} D_{1},{ }^{3} P_{1}$, and ${ }^{3} P_{0}$ levels are from Ref. [78], [79] and [80], respectively. 
upper levels of the transition, which is given by [83]:

$$
I_{s a t}=\frac{\pi h c \Gamma}{3 \lambda^{3} R_{b r}}
$$

where $R_{b r}$ is the branching ratio from the upper level to the lower level. As an example, for the ${ }^{2} S_{1 / 2} \leftrightarrow{ }^{2} P_{1 / 2}$ transition in $\mathrm{Yb}^{+}, \Gamma=1 / \tau=123.2 \mathrm{MHz}$ (measurement in Chap. VI), $\lambda=369.53 \mathrm{~nm}$, and $R_{b r}=1-0.005$ (measurement in Sec. 4.4), so from Eq. 4.2 we find $I_{\text {sat }}=51 \mathrm{~mW} / \mathrm{cm}^{2}{ }^{2}$ This means if the waist of the incident beam is $30 \mu \mathrm{m}$, then the power needed to saturate the transition is $1.4 \mu \mathrm{W}$.

Atoms propagating along or opposed to the incident light will see the frequency of this light as shifted, due to the Doppler effect. Since Eq. 4.1 is a function of the detuning $\Delta_{l}$, the scattering rate will depend on the velocity of the atom. By tuning the incident light to be red of resonance (photons lower in energy), an atom moving toward the light will scatter more photons than it does while moving away. Given the restoring force provided by the ion trap, this allows a trapped ion to be cooled. In fact, as long as the incident light is not aligned perpendicular to any of the principle axes of the trap, a single beam of incident light can cool the motion of the atom in all directions. The limit in temperature that can be achieved by Doppler cooling is set by the photon recoil energy. Although the atom scatters preferentially while moving in a particular direction, the spontaneous emission is still in a random direction, so any particular scattering event can heat the motion of the atom, rather than cool it. It can be shown that the minimum temperature is achieved at a detuning $\Delta_{l}=-\Gamma / 2$, resulting in [82]:

$$
k_{B} T=\frac{\hbar \Gamma}{2}
$$

In the case of the ${ }^{2} S_{1 / 2} \leftrightarrow{ }^{2} P_{1 / 2}$ transition in $\mathrm{Yb}^{+}$, this yields a Doppler cooling limit

\footnotetext{
${ }^{2}$ This assumes a two-level system. While assumption is approximately correct for the even isotopes of ytterbium (e.g. $\pi$-polarized light), for ${ }^{171} \mathrm{Yb}^{+}$we need to account for the effect of coherent dark states [84], as is done in Sec. 4.5.
} 


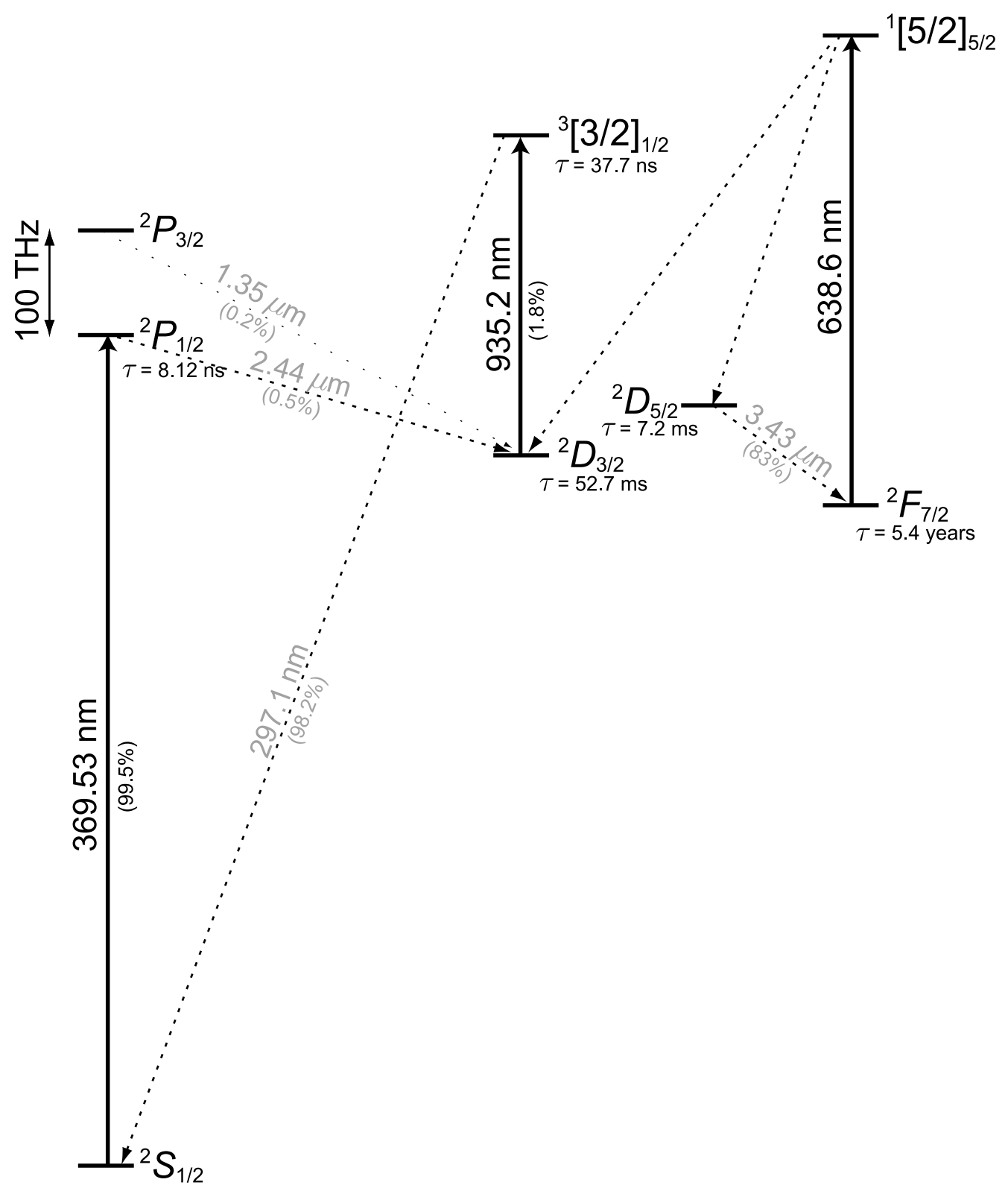

Figure 4.2: Relevant levels of the $\mathrm{Yb}^{+}$atom (to scale). Transitions shown with solid lines are driven by laser sources in the experiment. Numbers given in parentheses are branching ratios. The lifetimes of some of the excited states are also given. Measurement of the lifetime and branching ratio for the ${ }^{2} P_{1 / 2}$ level is given in Chap. VI and Sec. 4.4, respectively. Wavelengths for decays shown in gray are from Ref. [62]. Lifetimes of the ${ }^{2} D_{3 / 2},{ }^{2} D_{5 / 2}$, ${ }^{3}[3 / 2]_{1 / 2}$, and ${ }^{2} F_{7 / 2}$ levels are from Refs. [85], [86], [87], and [88], respectively. Branching ratios out of ${ }^{3}[3 / 2]_{1 / 2}$ and ${ }^{2} P_{3 / 2}$ are from Ref. [89], while the ${ }^{2} D_{5 / 2}$ branching ratio is from Ref. [86]. 
of $470 \mu \mathrm{K}$. In Chap. III, we derived the classical equations of motion for a charged particle in an rf trap, and found the particle underwent harmonic oscillations. Of course, since this a quantum system, we could instead derive quantized motion of the trapped particle [41]; but we know the energy of a quantum harmonic oscillator is given by $E_{h o}=\hbar \omega(n+1 / 2)$. Thus, given the secular frequency of a trapped ${ }^{171} \mathrm{Yb}^{+}$atom from Sec. 3.2.2 as $\omega_{x, \text { sim }} /(2 \pi)=1.17 \mathrm{MHz}$, and equating $k_{B} T$ in Eq. 4.3 to $E_{h o}$, we find the average motional state of a trapped ${ }^{171} \mathrm{Yb}^{+}$atom at the Doppler cooling limit to be $\bar{n}_{x} \approx 8$. The spatial extent of the atomic wavefunction is given by

$$
\left(\left\langle n\left|\hat{x}^{2}\right| n\right\rangle\right)^{1 / 2}=x_{0}(2 n+1)^{1 / 2}, \text { with } x_{o}=\sqrt{\frac{\hbar}{\left(2 m \omega_{x}\right)}}
$$

where $x_{o}$ is the "zero-point" spread of the wavefunction. Given the parameters for ${ }^{171} \mathrm{Yb}^{+}$above, at $\bar{n}_{x} \approx 8$ this leads to the wavefunction having a spatial extent of about $21 \mathrm{~nm}$.

In the experiment, we typically Doppler cool the $\mathrm{Yb}^{+}$atom on the ${ }^{2} S_{1 / 2} \leftrightarrow{ }^{2} P_{1 / 2}$ transition with about $500 \mathrm{nW}$ of $369.53 \mathrm{~nm}$ light focused to a $30 \mu \mathrm{m}$ waist. ${ }^{3}$ However, efficient cooling of the atom requires addtional light, because the ${ }^{2} P_{1 / 2}$ state also decays to the metastable ${ }^{2} D_{3 / 2}$ level with a measured probability of about 0.005 (see Sec. 4.4 for this measurement). Light at $935.2 \mathrm{~nm}$ is used to drive the atom from the ${ }^{2} D_{3 / 2}$ to the ${ }^{3}[3 / 2]_{1 / 2}$ level, from which it quickly returns to the ${ }^{2} S_{1 / 2}$ ground state [90]. An additional complication arises from the presence of the low-lying ${ }^{2} F_{7 / 2}$ state. Despite the fact that there are no allowed decays from the four levels used in cooling to ${ }^{2} F_{7 / 2}$, the ion falls into this state a few times per hour, probably due to collisions with residual background gas [91, 92]. Laser light near $638.6 \mathrm{~nm}$

\footnotetext{
${ }^{3}$ The $369.53 \mathrm{~nm}$ light generated by the frequency-doubling cavity is coupled into a single-mode fiber, and the output of this fiber is focused onto the ion. The spatial-mode filtering of the fiber allows for a very small waist to be achieved.
} 
depopulates the ${ }^{2} F_{7 / 2}$ level, returning the ion to the four-level cooling scheme.

The odd-looking notation of the ${ }^{3}[3 / 2]_{1 / 2}$ level is the result of a different coupling scheme (not the usual $L S$-coupling), caused by the excitation of a second electron from the $f$-shell of the ytterbium ion. In this coupling scheme, the $4 f^{13}$ shell is $L S$-coupled to yield a total $J_{c}$ for the core. The value $K$, given in brackets in the notation, is the result of coupling the orbital angular momentum of the two outer electrons $L$ to the core angular momentum $J_{c}$, so that $K=L+J_{c}$. The spins of the two outer electrons are coupled to form $S$, given by the superscript multiplicity $(2 S+1)$ in the notation. This value for $S$ is then coupled to $K$ to form the total $J$, given as the subscript in the notation $[93,94,62]$.

Photons at $369.53 \mathrm{~nm}$ scattered by the ion are collected by an objective lens with a numerical aperture of $0.27 .{ }^{4}$ An aperture at the intermediate image generated by the objective reduces observed background light. This image is re-imaged using a doublet lens and directed to either a photon-counting photomultiplier tube (PMT) or camera. The image provided by the camera is used to monitor the loading process and subsequently confirm the presence of a single $\mathrm{Yb}^{+}$atom in the trap. The PMT, with its higher detector efficiency, is used to measure the ion fluorescence during state detection.

The ion fluorescence can also be used to gauge residual micromotion at the rf drive frequency using a fluorescence cross-correlation technique [95]. Careful adjustment of static offset voltages applied to the trap rods aligns the equilibrium position of the ion to the center of the rf quadrupole potential, and suppresses micromotion [40].

\footnotetext{
${ }^{4}$ The objective is from Special Optics, part number 54-17-29-370nm.
} 


\subsection{Laser stabilization}

Efficient quantum operations on $\mathrm{Yb}^{+}$atoms require that the $739.05 \mathrm{~nm}$ and 935.2 nm lasers are stable in frequency to well within the linewidths of the relevant transitions in the experiment. We accomplish this by locking the $739.05 \mathrm{~nm}$ laser to a passively stable reference cavity using an rf (Pound-Drever-Hall) lock [96, 97]; the design of this invar cavity is given in App. C. Saturated-absorption spectrocopy of iodine is used for an absolute frequency reference to stabilize the length of the cavity. Lastly, we lock the $935.2 \mathrm{~nm}$ laser to the same cavity using a side-of-fringe technique.

The relevant optics and electronics for the rf stabilization of the $739.05 \mathrm{~nm}$ laser to the cavity are shown in Fig. 4.3(a). The current of the diode laser is weakly modulated at $120 \mathrm{MHz}$, and approximately $120 \mu \mathrm{W}$ of the main $739.05 \mathrm{~nm}$ beam is sent to the reference cavity. ${ }^{5}$ The cavity reflection is measured with a photodiode sensitive to rf signal modulation (about 1-150 MHz), and the consequent electronic signal is sent through an amplifier and mixed with the $120 \mathrm{MHz}$ signal. The resulting error signal is used to stabilize the $739.05 \mathrm{~nm}$ laser to the reference cavity by feedback control of the laser diode current and grating angle. The cavity is passively stable on short time-scales, and for long-term stability it is locked to an absorption line of molecular iodine.

Molecular iodine is often chosen as a frequency reference for wavelengths from the near-infrared (e.g. $830 \mathrm{~nm}[98]$ ) to the disassociation limit at $499.5 \mathrm{~nm}$ because of the density of narrow absorption lines in this region $[99,100]$. These lines can serve as excellent frequency references for laser stabilization to a few parts in $10^{-9}$ or

\footnotetext{
${ }^{5}$ The free spectral range of the doubling cavity prevents this modulation frequency from appearing on the 369.53 nm light.
} 

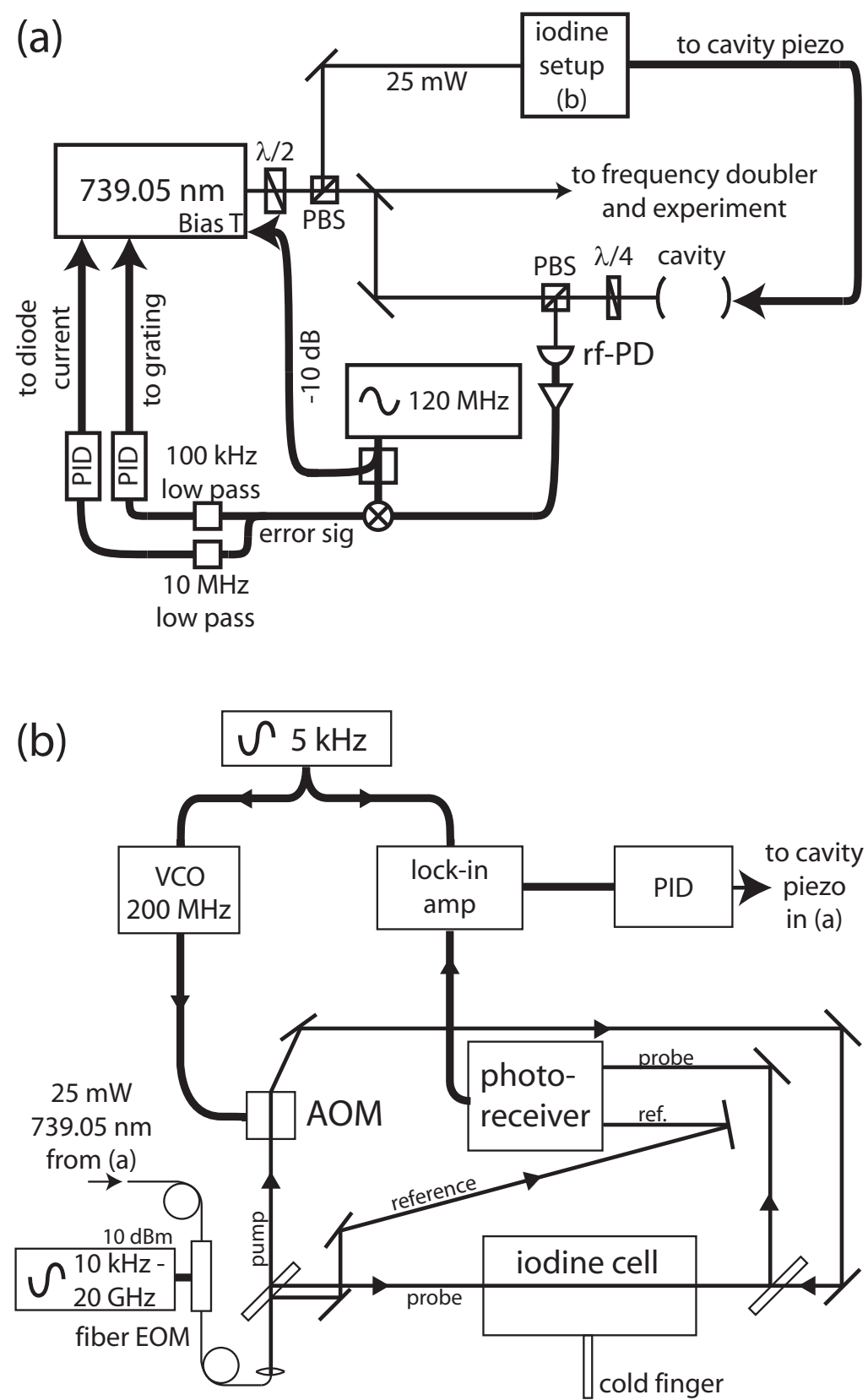

Figure 4.3: (a) Apparatus for locking the $739.05 \mathrm{~nm}$ laser to an iodine stabilized cavity, as explained in the text. The current of the diode laser is weakly modulated at $120 \mathrm{MHz}$ to lock the laser to a passively stable invar cavity. The cavity is stabilized to a reference line in molecule iodine. PID is proportional-integral-derivative servo controller; rf-PD is radio-frequency photodiode; $\mathrm{PBS}$ is a polarizing beamsplitter; $\lambda / 2$ is a half waveplate; and $\lambda / 4$ is a quarter waveplate. (b) Setup for locking to molecular iodine via saturated absorption spectroscopy, as explained in the text. The error signal generated from this setup is used to stabilize the reference cavity shown in (a). EOM is electro-optic modulator; AOM is acousto-optic modulator; VCO is voltage-controlled oscillator. 
better [101]. However, in the region applicable to $\mathrm{Yb}^{+}(739.05 \mathrm{~nm})$ most of the lines are weak at room temperature. In order to thermally achieve sufficient population of the rovibrational levels of the molecule that are the lower states of these $(B \rightarrow X)$ transitions, the iodine must be heated to over $600 \mathrm{~K}$ [102]. In our setup, we employ a homemade iodine vapor cell $20 \mathrm{~cm}$ in length with two quartz windows $25 \mathrm{~mm}$ in diameter and a $5 \mathrm{~cm}$ long cold finger. The cold finger is isolated from the heating elements such that it remains at room temperature, which is necessary to avoid pressure broadening of the transition [102].

The reference cavity is stabilized to molecular iodine using standard saturated absorption spectroscopy, as depicted in Figure 4.3(b). The $739.05 \mathrm{~nm}$ diode laser produces about $400 \mathrm{~mW}$ of output power, $25 \mathrm{~mW}$ of which is diverted to the iodine setup. This light is sent to a $10 \mathrm{kHz}-20 \mathrm{GHz}$ fiber electro-optic modulator (EOM), and one of the resulting first-order sidebands is used for iodine spectroscopy. ${ }^{6}$ At $739.05 \mathrm{~nm}, 25 \mathrm{~mW}$ of light can be safely sent through the EOM without photorefractive damage to the crystal. However, due to coupling and transmission losses, only $6 \mathrm{~mW}$ of this light is transmitted. The modulator receives approximately $10 \mathrm{dBm}$ of rf power, which transfers about $1 / 3$ of the power (effectively about $2 \mathrm{~mW}$ ) into the first-order sideband used for spectroscopy. Since the fraction of power transferred into the first-order sideband is given by $\left(J_{1}(\theta)\right)^{2}$, with $\theta$ the phase-shift induced by the modulating voltage, this is the maximum amount that can be expected. The remaining light at other frequencies is far detuned from any iodine feature, and therefore does not contribute to the absorption signal. A feature of this setup is that the large bandwidth of the fiber EOM allows the laser to be scanned over a wide range while remaining locked to a given iodine absorption line.

\footnotetext{
${ }^{6}$ EOSPACE Inc., Model PM-0K1-10-PFU-PFU-740-UL. Complete specifications are available at the EOSPACE website: http://www.eospace.com.
} 
We performed spectroscopy on three iodine reference lines to determine the best absorptive feature for use as an absolute frequency reference. Absorption lines of molecular iodine are observed at detunings of approximately $13 \mathrm{GHz}, 10 \mathrm{GHz}$, and $5 \mathrm{GHz}$ from the target wavelength of $739.05 \mathrm{~nm}$ (twice the wavelength of the ${ }^{2} S_{1 / 2} \leftrightarrow$ ${ }^{2} P_{1 / 2}$ transition of $\left.{ }^{171} \mathrm{Yb}^{+}\right) .{ }^{7}$ The continuous tuning of the fiber EOM over nearly $20 \mathrm{GHz}$ allowed us to perform spectroscopy to determine the hyperfine structure of these absorption lines. For this measurement, we stabilize the $739.05 \mathrm{~nm}$ laser to the fluorescence of a single ${ }^{174} \mathrm{Yb}^{+}$atom using a side-of-fringe technique. We estimate that this results in a stability of better than $5 \mathrm{MHz}$ over the course of the measurement. As the intensity of the $935.2 \mathrm{~nm}$ light on the ion was much greater than the saturation intensity $\left(\sim 20 \times I_{\text {sat }}\right)$ for this measurement, small fluctuations in the frequency of the $935.2 \mathrm{~nm}$ laser had a negligible effect on the ion fluorescence.

With the $739 \mathrm{~nm}$ laser locked to a trapped ion, the rf applied to the fiber EOM was varied in $0.5 \mathrm{MHz}$ steps over the areas of interest, and the output of the lock-in amplifier was recorded. Because of the weak nature of the lines being investigated, each point was integrated for about 1.5 seconds using a lock-in time constant of 300 ms to achieve a reasonable signal-to-noise ratio. We mapped the hyperfine structure of the three aforementioned absorption lines, with the hyperfine structure of the line near a detuning of $10 \mathrm{GHz}(739.03 \mathrm{~nm})$ presented in Fig. 4.4. Later, we further improved the signal by very careful alignment of the foci of the pump and probe beams in the iodine cell. The strong doublet feature at a detuning of approximately 10.3 GHz is used to stabilize the reference cavity of Fig. 4.3(a).

After the cavity has been stabilized to iodine, a weak (approximately $500 \mu \mathrm{W}$ )

\footnotetext{
${ }^{7}$ The absorption lines at detunings of approximately $13 \mathrm{GHz}, 10 \mathrm{GHz}$, and $-5 \mathrm{GHz}$ from the target wavelength of $739.05 \mathrm{~nm}$ are the features listed in the atlas by Gerstenkorn, et al. [99], at $13531.2773 \mathrm{~cm}^{-1}, 13531.1823 \mathrm{~cm}^{-1}$, and $13530.6745 \mathrm{~cm}^{-1}$, respectively.
} 


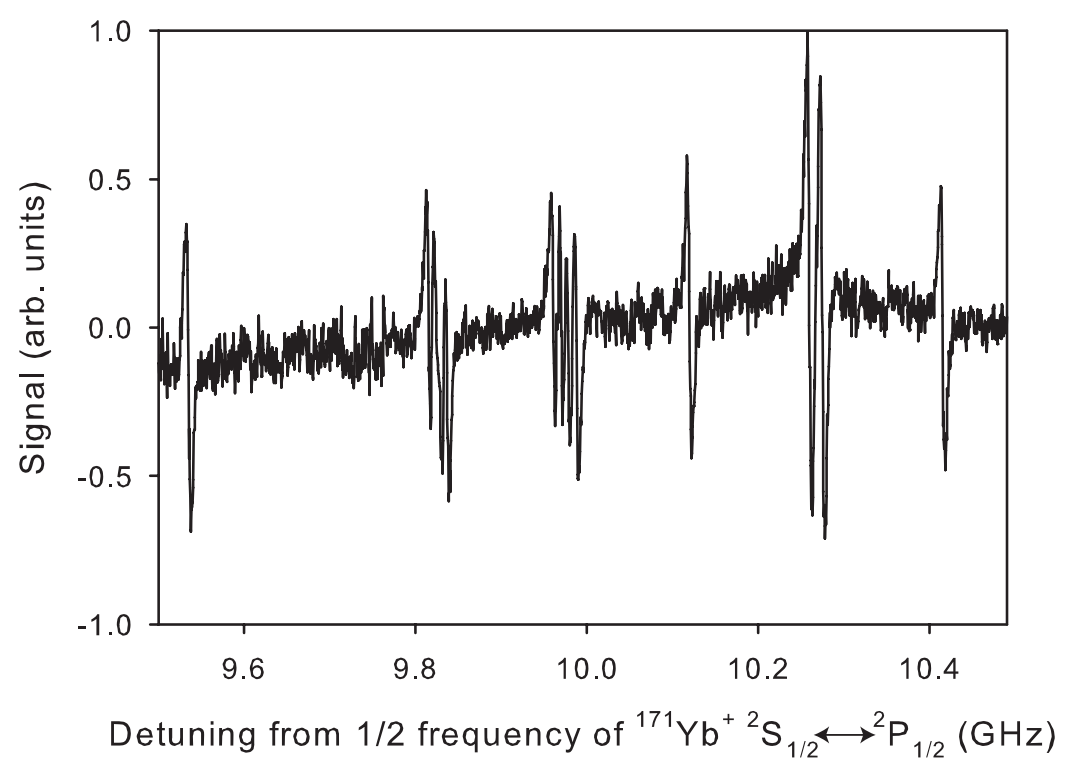

Figure 4.4: Hyperfine structure of the iodine absorption line approximately $10 \mathrm{GHz}$ detuned from half the frequency of the ${ }^{2} S_{1 / 2} \leftrightarrow{ }^{2} P_{1 / 2}$ transition of ${ }^{171} \mathrm{Yb}^{+}(739.05 \mathrm{~nm})$. Each point is separated in frequency by $0.5 \mathrm{MHz}$, and was integrated for about 1.5 seconds. The frequency axis in the figure is estimated to have an offset accurate to $\pm 20 \mathrm{MHz}$, and scaling to $\pm 5 \mathrm{MHz}$.

beam at $935.2 \mathrm{~nm}$ is sent through a fiber EOM and then directed (about $50 \mu \mathrm{W}$ ) to the cavity. The rf used to drive this fiber EOM is tuned such that one of the resulting first-order sidebands is resonant with the cavity. The transmitted light is observed with a photodiode, and the consequent signal sent to a PID controller. In this way, we stabilize the laser at $935.2 \mathrm{~nm}$ to the cavity using a side-of-fringe technique.

\subsection{Branching ratio of the ${ }^{2} P_{1 / 2}$ level}

We measure the ${ }^{2} P_{1 / 2}$ branching ratio to the ${ }^{2} D_{3 / 2}$ level by observing the decay in fluorescence of a single trapped ${ }^{174} \mathrm{Yb}^{+}$atom. As in Sec. 4.3, the $739.05 \mathrm{~nm}$ laser is stabilized to an absorption feature in iodine via a reference cavity. The 935.2 $\mathrm{nm}$ laser is stabilized to the same cavity, but here is tuned far (about $3 \mathrm{GHz}$ ) from the ${ }^{2} D_{3 / 2} \leftrightarrow{ }^{3}[3 / 2]_{1 / 2}$ transition. On the way to the ion, the $935.2 \mathrm{~nm}$ light is passed through a fiber EOM that produces a first-order sideband resonant with the 
${ }^{2} D_{3 / 2} \leftrightarrow{ }^{3}[3 / 2]_{1 / 2}$ transition of ${ }^{174} \mathrm{Yb}^{+}$.

The measurement sequence begins with a $100 \mu$ s interval during which the fiber EOM modulating the $935.2 \mathrm{~nm}$ light is on, and both the $935.2 \mathrm{~nm}$ light and 369.53 $\mathrm{nm}$ light are incident on the ion. In a subsequent interval of $95 \mu \mathrm{s}$, the fiber EOM modulating the $935.2 \mathrm{~nm}$ light is switched off, resulting in population trapping in ${ }^{2} D_{3 / 2}$. During this time, photons scattered by the ion are collected and sent to the PMT, with the arrival times of the photons recorded by a time-to-digital converter. The fluorescence signal has an exponential decay arising from population trapping in the ${ }^{2} D_{3 / 2}$ state, with functional form $\exp \left(-\mathcal{P}_{P_{1 / 2}} \Gamma R_{b r} t\right)$. Here, $\Gamma$ is the spontaneous emission rate of the ${ }^{2} P_{1 / 2}$ state; $R_{b r}$ is the branching ratio (probability of decay) into ${ }^{2} D_{3 / 2}$; and $\mathcal{P}_{P_{1 / 2}}$ is the population of the ${ }^{2} P_{1 / 2}$ state, which is a function of the power $(p)$ of the $369.53 \mathrm{~nm}$ light at the ion (for a constant beam waist), given by $\mathcal{P}_{P_{1 / 2}}=\left(p / p_{\text {sat }}\right) /\left(2\left(1+p / p_{\text {sat }}\right)\right)$ for resonant light. Decay out of the metastable ${ }^{2} D_{3 / 2}$ state is neglected because the lifetime of this state (52.7 ms [85]) is much longer than the measurement time.

Figure 4.5(a) shows this exponential decay for $29 \mu \mathrm{W}$ of incident $369.53 \mathrm{~nm}$ light, focused to a waist of approximately $30 \mu \mathrm{m}$ at the ion. We repeat the measurement for a variety of $369.53 \mathrm{~nm}$ light intensities, with the resulting decay parameters $b=\mathcal{P}_{P_{1 / 2}} \Gamma R_{b r}$ shown in Fig. 4.5(b). Given the expressions for $b$ and $\mathcal{P}_{P_{1 / 2}}$ above, the data is fit to $p=\left(2 b p_{s a t}\right) /\left(\Gamma R_{b r}-2 b\right)$ with two fit parameters: $p_{s a t}$ and $\Gamma R_{b r}$. Using $\Gamma=1 /\left(8.12 \pm 0.02 \mathrm{~ns}\right.$ ) (see Chap. VI), we determine the ${ }^{2} P_{1 / 2}$ branching ratio to ${ }^{2} D_{3 / 2}$ to be $R_{b r}=0.0050(2)$. This number is consistent with prior theoretical calculations [103] and experimental measurements [85]. Knowledge of this branching ratio can be important in determining the theoretical limit on state detection of an $\mathrm{Yb}^{+}$qubit, as discussed in Sec. 4.5. 

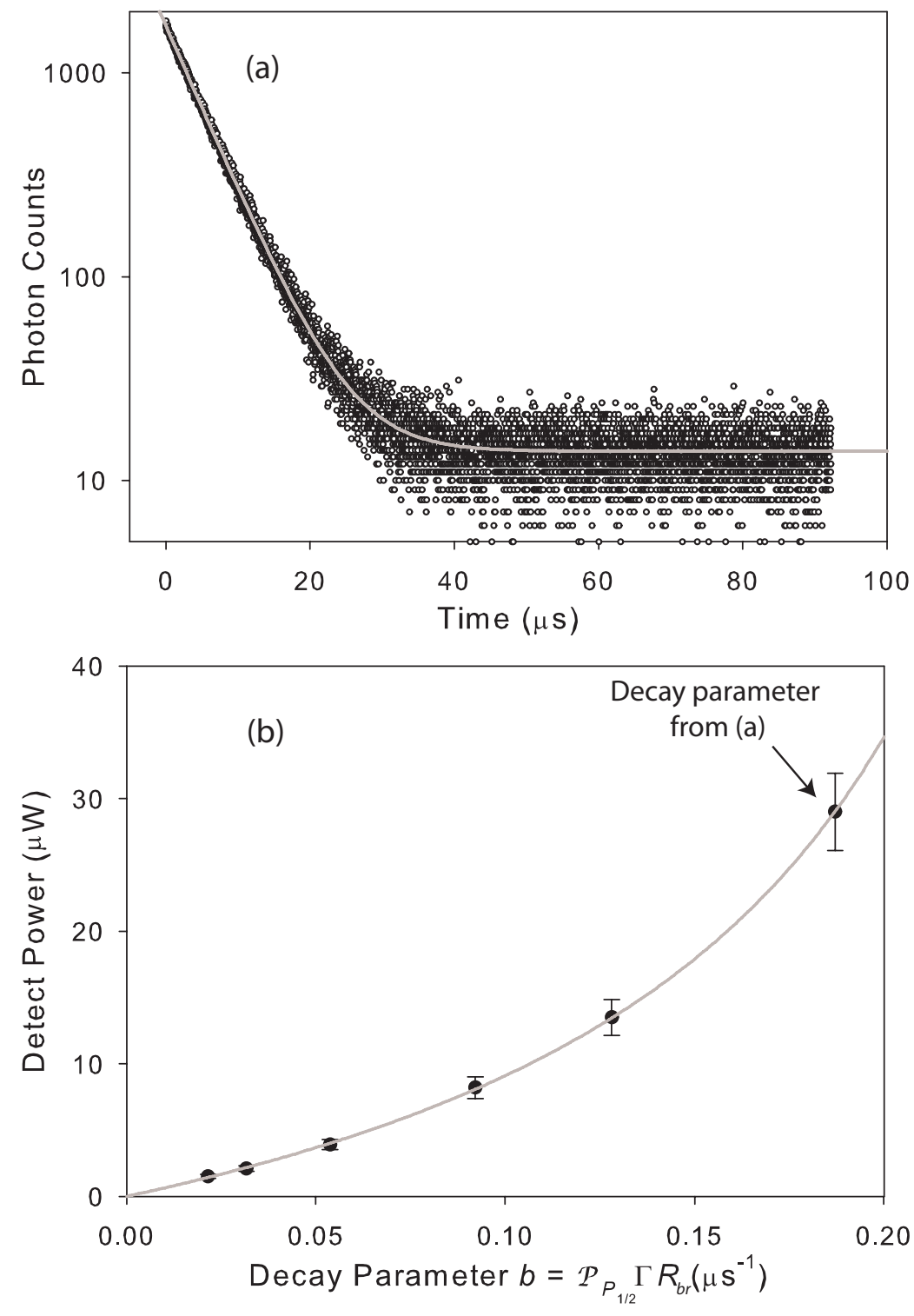

Figure 4.5: Data used to determine the branching ratio of the ${ }^{2} P_{1 / 2}$ level. (a) Exponential decay in the fluorescence of a trapped ${ }^{174} \mathrm{Yb}^{+}$atom, indicating population trapping in ${ }^{2} D_{3 / 2}$. During this time, appproximately $29 \mu \mathrm{W}$ of $369.53 \mathrm{~nm}$ light was incident on the ion, with the $935.2 \mathrm{~nm}$ laser detuned about $3 \mathrm{GHz}$ from the ${ }^{2} D_{3 / 2} \leftrightarrow{ }^{3}[3 / 2]_{1 / 2}$ transition. The data was integrated for 5 minutes, and is analyzed with $16 \mathrm{~ns}$ binning. The gray line is an exponential (plus background) fit to the data. (b) Exponential fit decay parameters $b=\mathcal{P}_{P_{1 / 2}} \Gamma R_{b r}$ for a range of $369.53 \mathrm{~nm}$ laser intensities. Error bars are taken to be $\pm 10 \%$ the measured $369.53 \mathrm{~nm}$ power, to account for possible intensity drifts of the laser during the measurement. The line is a fit to the function $p=\left(2 b p_{s a t}\right) /\left(\Gamma R_{b r}-2 b\right)$ with two fit parameters: $p_{s a t}$ and $\Gamma R_{b r}$. 


\subsection{Hyperfine qubit}

As discussed in Chap. II, the requirements for the physical implementation of quantum computation include state initialization of the qubits, qubit state detection, a set of universal gates, and long qubit coherence time [29]. The ${ }^{2} S_{1 / 2}$ hyperfine levels of the ${ }^{171} \mathrm{Yb}^{+}$atom satisfy all of these requirements, making it an excellent qubit for quantum information processing. Below, we show that with knowledge of the hyperfine splitting of the relevant energy levels and the application of additional frequency sources, we are able to achieve fast, efficient state initialization and state detection, reliable single-qubit operations, and a qubit coherence time that far exceeds the duration of these processes.

The relevant energy levels for the ${ }^{171} \mathrm{Yb}^{+}$atom are shown in Fig. 4.6. The qubit is defined to be the two first-order magnetic field-insensitive hyperfine levels of the ${ }^{2} S_{1 / 2}$ ground state. We define the ${ }^{2} S_{1 / 2}\left|F=1, m_{F}=0\right\rangle$ state as the logical qubit state $|1\rangle$, and the ${ }^{2} S_{1 / 2}\left|F=0, m_{F}=0\right\rangle$ state as $|0\rangle$. Here $F$ is the total angular momentum of the atom and $m_{F}$ is its projection along the quantization axis. The qubit states are separated by a frequency of $12642812118.5+\delta \mathrm{Hz}$, where $\delta=(310.8) B^{2}$ is the second-order Zeeman shift, and $B$ is the magnetic field in gauss [35].

4.5.1 Doppler cooling ${ }^{171} \mathbf{Y b}^{+}$The ion is Doppler-cooled in a way very similar to that presented in Sec. 4.2, with the difference arising from the addition of the hyperfine structure of the 171 isotope. In this case, light at $369.53 \mathrm{~nm}$ is slightly red-detuned (about $10 \mathrm{MHz}$ ) of the ${ }^{2} S_{1 / 2}|F=1\rangle \leftrightarrow{ }^{2} P_{1 / 2}|F=0\rangle$ transition in ${ }^{171} \mathrm{Yb}^{+}$. During cooling, there is approximately $500 \mathrm{nW}$ of $369.53 \mathrm{~nm}$ light focused to a waist of about $30 \mu \mathrm{m}$ at the ion. Off-resonant coupling to the ${ }^{2} P_{1 / 2}|F=1\rangle$ manifold results in population trapping in $|0\rangle$. To prevent this during cooling intervals, the $369.53 \mathrm{~nm}$ 


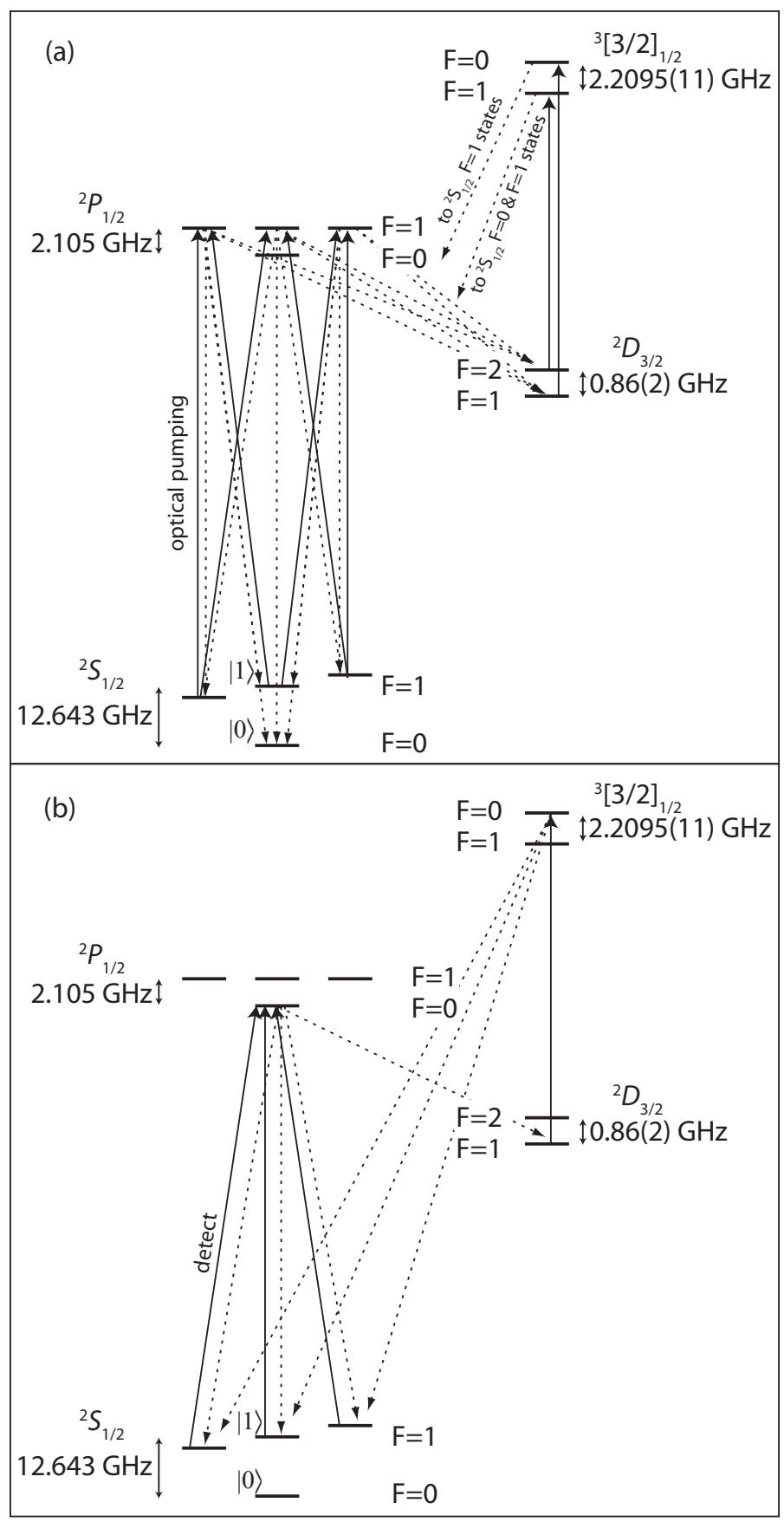

Figure 4.6: The ${ }^{171} \mathrm{Yb}^{+}$qubit (not to scale). The ${ }^{2} S_{1 / 2}\left|F=1, m_{F}=0\right\rangle$ state is defined to be $|1\rangle$, and the ${ }^{2} S_{1 / 2}\left|F=0, m_{F}=0\right\rangle$ state is defined to be $|0\rangle$. (a) State initialization to $|0\rangle$ by application of light resonant with the ${ }^{2} S_{1 / 2}|F=1\rangle \leftrightarrow{ }^{2} P_{1 / 2}|F=1\rangle$ transition. (b) Detection of the qubit state. If the qubit state is $|1\rangle$, the $369.53 \mathrm{~nm}$ light applied for detection is nearly on resonance, and the ion scatters many photons. If the state is $|0\rangle$, very few photons are scattered. Measurements of the hyperfine splitting of the ${ }^{2} S_{1 / 2}$ and ${ }^{2} P_{1 / 2}$ levels are found in Ref. [35] and [104], respectively. Details regarding our measurement of the hyperfine splitting of ${ }^{2} D_{3 / 2}$ and ${ }^{3}[3 / 2]_{1 / 2}$ are found in Sec. 4.6. 
cooling beam is passed through a bulk resonant EOM driven at $7.37 \mathrm{GHz}$, as shown in

Fig. 4.7. The resulting positive second-order sideband is resonant with the ${ }^{2} S_{1 / 2} \mid F=$ $0\rangle \leftrightarrow{ }^{2} P_{1 / 2}|F=1\rangle$ transition, which returns the ion to the cooling cycle. ${ }^{8}$ Population trapping in the ${ }^{2} D_{3 / 2}|F=1\rangle$ manifold is avoided by application of a laser at $935.2 \mathrm{~nm}$, which rapidly returns the atom to the cooling cycle via the ${ }^{3}[3 / 2]_{1 / 2}|F=0\rangle$ level [90]. As seen in Fig. 4.2 , the ${ }^{3}[3 / 2]_{1 / 2}$ decays preferentially to ${ }^{2} S_{1 / 2}$ [89]. About $600 \mu \mathrm{W}$ of $935.2 \mathrm{~nm}$ light is sent to the trap, focused to a spot with waist approximately $200 \mu \mathrm{m}$. When the ion is excited to the ${ }^{2} P_{1 / 2}|F=1\rangle$ manifold, which occurs off-resonantly during cooling, the ion may decay to ${ }^{2} D_{3 / 2}|F=2\rangle$. We depopulate this level by using a fiber EOM driven at $3.07 \mathrm{GHz}$ to add a frequency component to the $935.2 \mathrm{~nm}$ light that is resonant with the ${ }^{2} D_{3 / 2}|F=2\rangle \leftrightarrow^{3}[3 / 2]_{1 / 2}|F=1\rangle$ transition (see Sec. 4.6). Finally, the ${ }^{2} F_{7 / 2}$ hyperfine levels are depopulated by laser light that is switched between the ${ }^{2} F_{7 / 2}|F=3\rangle \leftrightarrow{ }^{1}[5 / 2]_{5 / 2}|F=2\rangle$ and ${ }^{2} F_{7 / 2}|F=4\rangle \leftrightarrow{ }^{1}[5 / 2]_{5 / 2}|F=3\rangle$ transitions, both near $638.6 \mathrm{~nm}$ [81]. Nearly $1 \mathrm{~mW}$ of $638.6 \mathrm{~nm}$ light is present at the trap, focused to about $200 \mu \mathrm{m}$. The ion resides in a magnetic field of about 3.4 gauss for definition of the quantization axis and to avoid coherent population trapping [84].

4.5.2 State initialization Optical pumping initializes the state of the ${ }^{171} \mathrm{Yb}^{+}$atom into $|0\rangle$, as shown in Fig. 4.6(a). A beam at $369.53 \mathrm{~nm}$ is passed through a bulk resonant $2.1 \mathrm{GHz}$ EOM before reaching the ion. During state initialization, this 2.1 GHz EOM is switched on, generating a positive first-order sideband resonant with the ${ }^{2} S_{1 / 2}|F=1\rangle \leftrightarrow{ }^{2} P_{1 / 2}|F=1\rangle$ transition. ${ }^{9}$ From the ${ }^{2} P_{1 / 2}|F=1\rangle$ manifold, the ion

\footnotetext{
${ }^{8}$ The EOM is New Focus model 4851; the frequency is generated by an HP $8684 \mathrm{D}$, and then amplified to send approximately $1 \mathrm{~W}$ to the EOM (max spec. power is $3 \mathrm{~W}$ ). This transfers only about $1 \%$ of the power into each of the second-order sidebands, limited by technical difficulties with the EOM. Nevertheless, this appears to be sufficient.

${ }^{9}$ The $2.1 \mathrm{GHz}$ EOM is from New Focus, model 4431. The driving frequency is provided by a PTS 3200 and is amplified before reaching the EOM. Approximately $0.5 \mathrm{~W}$ of $\mathrm{rf}$ power is applied to the EOM, which almost completely suppresses the carrier (max spec. power is $4 \mathrm{~W}$ ).
} 


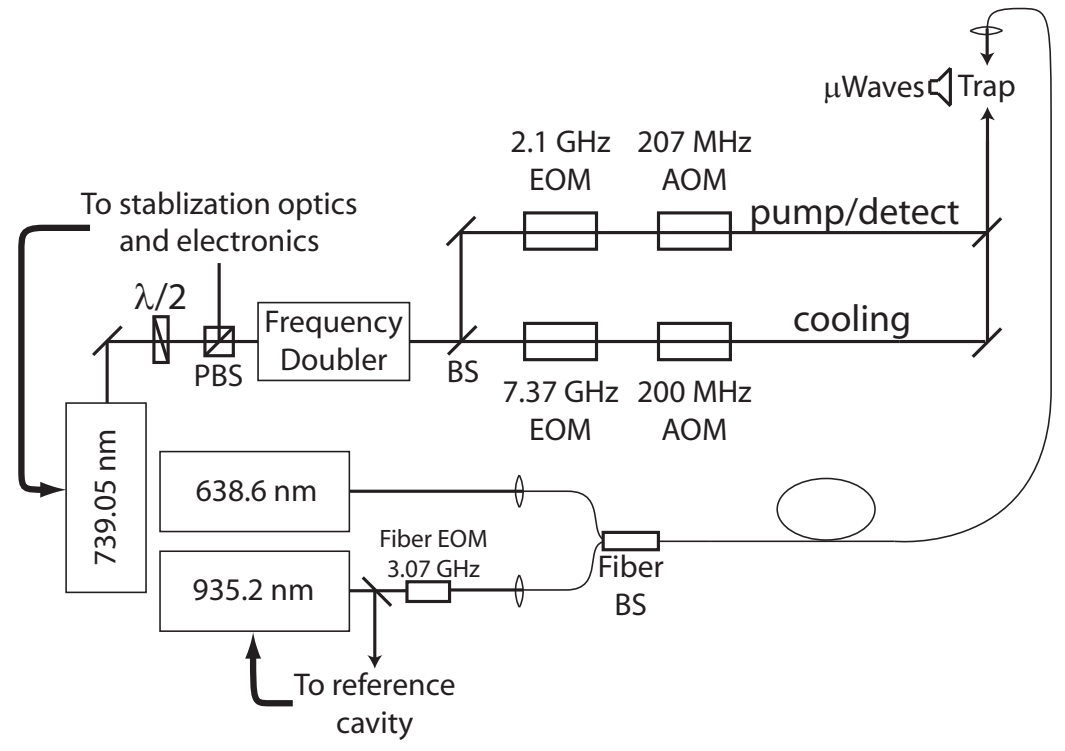

Figure 4.7: Experimental setup for initialization, manipulation, and detection of the ${ }^{171} \mathrm{Yb}^{+}$qubit. The 7.37 GHz EOM in the cooling beam is tuned so that the positive second-order sideband is on resonance with the ${ }^{2} S_{1 / 2}|F=0\rangle \leftrightarrow{ }^{2} P_{1 / 2}|F=1\rangle$ transition, preventing population trapping in $|0\rangle$ during cooling. The EOM in the pump/detect beam at $2.1 \mathrm{GHz}$ is used to optically pump the ion into $|0\rangle$ during state initialization. The $935.2 \mathrm{~nm}$ beam passes through a widely tunable fiber EOM, which is used to generate a frequency component to depopulate the ${ }^{2} D_{3 / 2}|F=2\rangle$ manifold during cooling and optical pumping. The AOMs are used to quickly turn the beams on and off. The AOM in the cooling beam is tuned such that the diffracted beam is about half a linewidth (about $10 \mathrm{MHz}$ ) away from resonance to achieve efficient cooling. The pump/detect beam is tuned only about $3 \mathrm{MHz}$ from resonance to generate more scattering events, reducing the time needed to distinguish between qubit states with high fidelity. Microwaves ( $\mu$ Waves) at about $12.643 \mathrm{GHz}$ are sent to the trap for single-qubit manipulation. 
has a $1 / 3$ chance of decaying to $|0\rangle$. Since ${ }^{2} P_{1 / 2}|F=1\rangle$ also decays to ${ }^{2} D_{3 / 2}|F=2\rangle$, the aforementioned $3.07 \mathrm{GHz}$ sideband in the $935.2 \mathrm{~nm}$ light is essential for efficient state initialization. With approximately $8 \mu \mathrm{W}$ of $369.53 \mathrm{~nm}$ light focused to about $30 \mu \mathrm{m}$ at the trap, the ion is optically pumped to the $|0\rangle$ state in less than $1 \mu$ s with near perfect efficiency.

4.5.3 State detection A critical step in quantum computation and communication protocols is detection of the qubit state. In ${ }^{171} \mathrm{Yb}^{+}$, state detection is accomplished using standard ion fluorescence techniques, as illustrated in Fig. 4.6(b) [41, 105, 106]. The $369.53 \mathrm{~nm}$ light of the detect beam is tuned to be nearly on resonance with the ${ }^{2} S_{1 / 2}|F=1\rangle \leftrightarrow{ }^{2} P_{1 / 2}|F=0\rangle$ transition. If the ion is prepared in the state $|0\rangle$, this incident light is detuned from ${ }^{2} P_{1 / 2}|F=1\rangle$ by $14.7 \mathrm{GHz}$, and thus the ion scatters very few photons. Conversely, if the $|1\rangle$ state is prepared, then the impinging light is nearly on resonance, and many scattered photons are observed. The state of the ion is determined by the number of photons observed by the PMT during the detection interval. In the experiments presented here, if more than one photon is observed during detection, the ion is defined to be in the state $|1\rangle$; if one or zero photons are detected, the ion is defined to be in $|0\rangle$.

The limiting source of error in state detection is off-resonant coupling to the ${ }^{2} P_{1 / 2}|F=1\rangle$ manifold. A detailed study of the theoretical detection fidelity limit for different ion species is presented in Ref. [106]. Given a photon collection efficiency of 0.001 (one out of every 1000 scattered photons is collected and detected) in the limit of low intensity of the detect beam, the maximum detection fidelity is calculated to be $99.52 \%$ for ${ }^{171} \mathrm{Yb}^{+} .{ }^{10}$ The majority of the error is a consequence of off-resonant excitation to the ${ }^{2} P_{1 / 2}|F=1\rangle$ manifold when the ion has been prepared in $|1\rangle$, as

\footnotetext{
${ }^{10}$ In Ref. [106], a theoretical detection efficiency of $99.33 \%$ is given. The discrepancy here arises from using different values for the lifetime of the ${ }^{2} P_{1 / 2}$ excited state. In present calculation, we use $19.6 \mathrm{MHz}$, as measured in Chap. VI.
} 


\begin{tabular}{ll}
\hline \hline & Theoretical \\
Photon collection efficiency & state detection fidelity \\
\hline 0.001 & $98.58 \%$ \\
0.002 & $99.28 \%$ \\
0.003 & $99.52 \%$ \\
0.01 & $99.86 \%$ \\
0.02 & $99.93 \%$ \\
0.03 & $99.95 \%$ \\
0.1 & $99.985 \%$ \\
\hline \hline
\end{tabular}

Table 4.3: Theoretical state detection fidelity in ${ }^{171} \mathrm{Yb}^{+}$for various photon collection efficiencies when the reduced scattering rate due to coherent dark states is included.

the hyperfine splitting of the ${ }^{2} P_{1 / 2}$ level is only $2.1 \mathrm{GHz}$. On the other hand, if the initial state is $|0\rangle$, the incident light is detuned from the ${ }^{2} P_{1 / 2}|F=1\rangle$ manifold by $14.7 \mathrm{GHz}$ and transitions to the ${ }^{2} P_{1 / 2}|F=0\rangle$ level are forbidden by selection rules. Taking into account decay into the ${ }^{2} D_{3 / 2}$ level in this calculation reduces the total number of $369.53 \mathrm{~nm}$ photons scattered by the ion. In addition, off-resonant coupling to the ${ }^{3}[3 / 2]_{1 / 2}|F=1\rangle$ level may occur while depopulating the ${ }^{2} D_{3 / 2}|F=1\rangle$ manifold. However, given the small branching ratio into ${ }^{2} D_{3 / 2}$, we estimate these additions to the calculation constitute a negligible change in the theoretical detection fidelity $(<0.01 \%)$. A far more important issue is the reduced scattering rate of the ${ }^{171} \mathrm{Yb}^{+}$atom due to coherent population trapping in the ${ }^{2} S_{1 / 2}|F=1\rangle$ manifold [84]. In optimal conditions, the scattering rate of the ${ }^{2} S_{1 / 2}|F=1\rangle \leftrightarrow{ }^{2} P_{1 / 2}|F=0\rangle$ transition used for detection is reduced to about $1 / 3$ the natural rate, while the error rate remains virtually unchanged. Hence, for a photon collection efficiency of 0.001 , the theoretical detection fidelity is reduced to about $98.58 \%$ (Table 4.3 ).

We experimentally optimize state detection with respect to the intensity and duration of the light applied during the detect interval. As a result of the small branching ratio into ${ }^{2} D_{3 / 2}$, we observe that the fidelity of state detection has negligible dependence on the $935.2 \mathrm{~nm}$ intensity (above $500 \mu \mathrm{W}$, focused to about $200 \mu \mathrm{m}$ ). The $369.53 \mathrm{~nm}$ light was varied over a wide range of intensities and durations. Optimum 


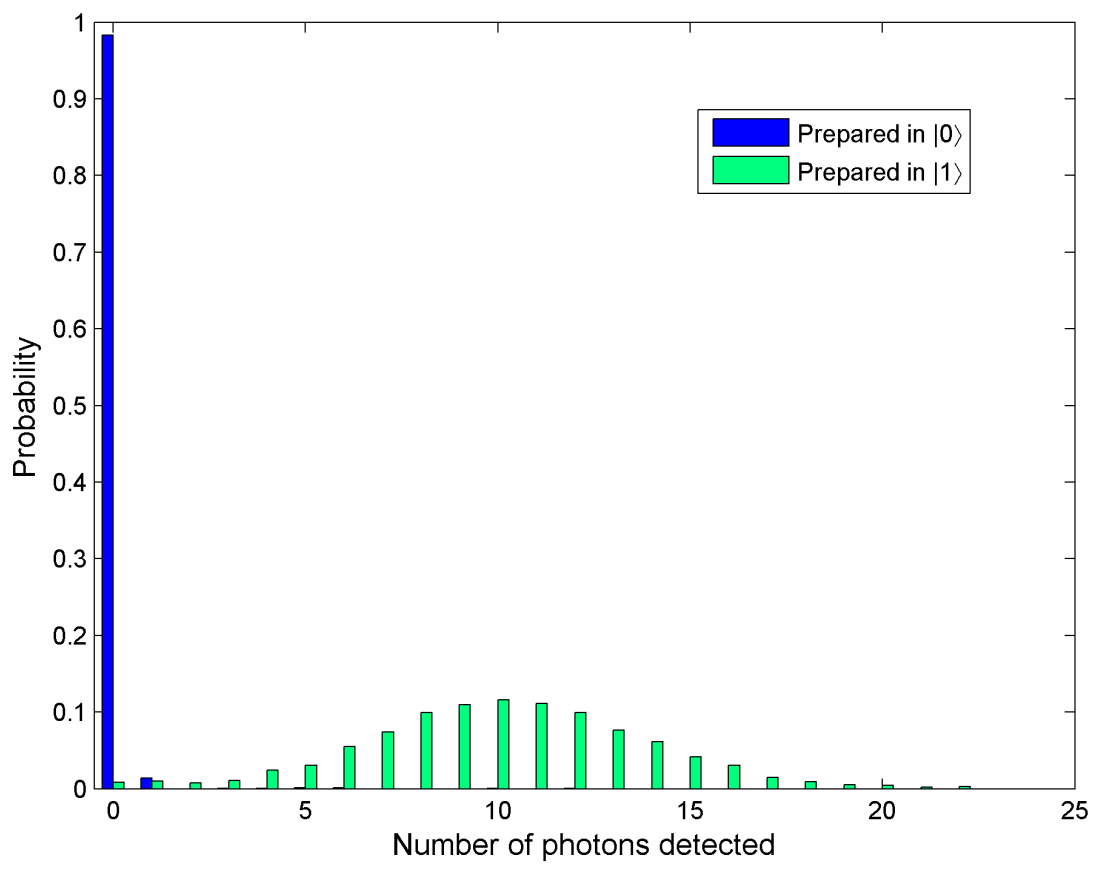

Figure 4.8: Histograms of detected photons after the ion is prepared in each of the two qubit states. The detection interval is $800 \mu \mathrm{s}$, with $150 \mathrm{nW}$ of $369.53 \mathrm{~nm}$ light focused to about 30 $\mu \mathrm{m}$. If zero or one photon is detected, the state is determined to be $|0\rangle$; if two or more photons, $|1\rangle$. Discriminating between the qubit states by this method yields a state detection fidelity of $98.2(2) \%$. Blue histogram: the ion is initialized to the $|0\rangle$ state, and the atom scatters very few photons. The histogram is the result of 5000 measurements. Green histogram: the ion is initialized to $|0\rangle$, and then rotated to $|1\rangle$ by application of microwaves. During the detection interval the atom scatters many photons. The histogram is the result of 4000 measurements.

state detection was realized with $150 \mathrm{nW}$ of $369.53 \mathrm{~nm}$ light, focused to a spot of radius about $30 \mu \mathrm{m}$ at the trap, and incident on the ion for $800 \mu \mathrm{s}$. The resulting histograms for state preparation in $|0\rangle$ versus $|1\rangle$ are presented in Fig. 4.8. Given these parameters, the state detection fidelity is measured to be $98.2(2) \%$.

4.5.4 Microwave rotations We can rotate the state of the ion between $|0\rangle$ and $|1\rangle$ by applying microwaves resonant with the $12.643 \mathrm{GHz}^{2} S_{1 / 2}$ hyperfine splitting. The microwave radiation effectively adds the perturbative Hamiltonian

$$
\hat{H}_{I}=-\hat{\mu}_{z} B_{o} \cos \left(\omega t+\delta_{o}\right)
$$


where we've assumed the polarization of the applied oscillating magnetic field is along the $z$-axis. Here, $\hat{\mu}_{z}$ is the $z$-component of the magnetic moment of the atom, $\omega$ is the angular frequency of the applied radiation, $B_{o}$ is the amplitude of the radiation, and $\delta_{o}$ is a phase. We assume this is only a small perturbation to the system, and expand the wavefunction of the system in terms of the eigenstates of the unperturbed Hamiltonian.

$$
|\psi(t)\rangle=\sum_{m} c_{m}(t) e^{-i E_{m} t / \hbar}|m\rangle
$$

Recall the time-dependent Schrödinger equation is

$$
i \hbar \frac{d}{d t}|\psi(t)\rangle=\hat{H}|\psi(t)\rangle
$$

where we've defined the Hamiltonian as the sum of the unperturbed and perturbation Hamiltonians, $\hat{H}=\hat{H}_{o}+\hat{H}_{I}$, with $\hat{H}_{o}|m\rangle=E_{m}|m\rangle$. Plugging Eq. 4.6 into Eq. 4.7 and taking the inner product with $\langle n|$ yields a set of coupled differential equations for the time-dependent coefficients.

$$
i \hbar \dot{c}_{n} e^{-i E_{n} t / \hbar}=\sum_{m} c_{m} e^{-i E_{m} t / \hbar}\left\langle n\left|\hat{H}_{I}\right| m\right\rangle
$$

Now we make the magnetic dipole approximation, which amounts to assuming that the wavelength of the magnetic radiation is much larger than the spatial extent of the atomic wavefunction; given our earlier estimate for the spatial extent of a trapped ion (Sec. 4.2), and a wavelength $c /(12.643 \mathrm{GHz})=2.37 \mathrm{~cm}$, this is an excellent approximation. The magnetic dipole approximation lets us write $\left\langle n\left|\hat{H}_{I}\right| m\right\rangle=$ $-B_{o} \cos \left(\omega t+\delta_{o}\right)\left\langle n\left|\hat{\mu}_{z}\right| m\right\rangle$. Since $\hat{\mu}_{z}$ is a vector quantity, it has odd parity, which means $\left\langle n\left|\hat{\mu}_{z}\right| m\right\rangle$ will be non-zero only for $n \neq m$. For simplicity, we will define the number $\wp_{n m}=\left\langle n\left|\hat{\mu}_{z}\right| m\right\rangle$; note that $\wp_{n m}=\wp_{m n}^{*}$.

Our interest is transitions in the two-level system $|0\rangle$ and $|1\rangle$. In this case, Eq. 4.8 
yields two coupled differential equations:

$$
\begin{aligned}
i \hbar \dot{c}_{0} & =-c_{1} \wp_{10} B_{o} e^{-i \omega_{10} t} \cos \left(\omega t+\delta_{o}\right) \\
i \hbar \dot{c}_{1} & =-c_{0} \wp_{10}^{*} B_{o} e^{i \omega_{10} t} \cos \left(\omega t+\delta_{o}\right)
\end{aligned}
$$

where we have defined $\omega_{10}=\left(E_{1}-E_{0}\right) / \hbar$. Rewriting the cosine as a sum of exponentials yields:

$$
\begin{aligned}
i \hbar \dot{c}_{0} & =-\frac{1}{2} c_{1} \wp_{10} B_{o}\left(e^{i\left(\omega-\omega_{10}\right) t} e^{i \delta_{o}}+e^{-i\left(\omega+\omega_{10}\right) t} e^{-i \delta_{o}}\right) \\
i \hbar \dot{c}_{1} & =-\frac{1}{2} c_{0} \wp_{10}^{*} B_{o}\left(e^{i\left(\omega+\omega_{10}\right) t} e^{i \delta_{o}}+e^{-i\left(\omega-\omega_{10}\right) t} e^{-i \delta_{o}}\right)
\end{aligned}
$$

We will now make the rotating wave approximation (RWA), the essence of which is that the dynamics of the amplitudes change slowly with respect to the incident frequency $\omega$. Given the applied radiation is near resonance, this assumption results in $e^{ \pm i\left(\omega+\omega_{10}\right) t} \approx 0$. If we also define the resonant Rabi frequency $\Omega=\wp_{10} B_{o} / \hbar$, and the detuning of the field from resonance as $\Delta=\omega-\omega_{10}$, the coupled differential equations are simplified to:

$$
\begin{aligned}
& \dot{c}_{0}=\frac{i \Omega}{2} c_{1} e^{i \Delta t} e^{i \delta_{o}} \\
& \dot{c}_{1}=\frac{i \Omega^{*}}{2} c_{0} e^{-i \Delta t} e^{-i \delta_{o}}
\end{aligned}
$$

Taking a second derivative of Eq. 4.12:

$$
\ddot{c}_{1}=\frac{i \Omega^{*}}{2} e^{-i \Delta t} e^{-i \delta_{o}}{\dot{c_{0}}}+(-i \Delta) \frac{i \Omega^{*}}{2} c_{0} e^{-i \Delta t} e^{-i \delta_{o}}
$$

and plugging-in Eq. 4.11 and Eq. 4.12 for the first and second terms on the right-hand side of the equation, respectively, results in a second-order, homogenous differential equation.

$$
\ddot{c}_{1}+i \Delta \dot{c}_{1}+\left|\frac{\Omega}{2}\right|^{2} c_{1}=0
$$


The general solution for this equation can be obtained by defining the operator $D=\frac{d}{d t}$, so that the equation becomes

$$
\left(D^{2}+i \Delta D+\left|\frac{\Omega}{2}\right|^{2}\right) c_{1}=0
$$

The "characteristic equation", $D^{2}+i \Delta D+\left|\frac{\Omega}{2}\right|^{2}=0$, is then solved for the quadratic roots $\alpha_{r}$ and $\beta_{r}$. Solving in this manner results in $\alpha_{r}=\frac{-i}{2}\left(\Delta+\sqrt{\Omega^{2}+\Delta^{2}}\right)$ and $\beta_{r}=\frac{-i}{2}\left(\Delta-\sqrt{\Omega^{2}+\Delta^{2}}\right)$. For reasons that will become apparent in the final solution, we will define the generalized Rabi frequency $\Omega_{R}=\sqrt{\Omega^{2}+\Delta^{2}}$. The general solution to Eq. 4.15 is then given by [107]:

$$
\begin{aligned}
c_{1}(t) & =a e^{\alpha_{r} t}+b e^{\beta_{r} t} \\
& =a e^{-i\left(\Delta+\Omega_{R}\right) t / 2}+b e^{-i\left(\Delta-\Omega_{R}\right) t / 2} \\
& =e^{-i \Delta t / 2}\left(a e^{-i \Omega_{R} t / 2}+b e^{i \Omega_{R} t / 2}\right)
\end{aligned}
$$

The constant coeffients $a$ and $b$ are determined by the initial conditions.

First, let's assume the atom has been initialized to $|0\rangle$ by optical pumping at time $t=0$, giving the initial conditions $c_{0}(0)=1$ and $c_{1}(0)=0$. The latter condition on Eq. 4.16 determines $b=-a$, so we have:

$$
\begin{aligned}
c_{1}(t) & =a e^{-i \Delta t / 2}\left(e^{-i \Omega_{R} t / 2}-e^{i \Omega_{R} t / 2}\right) \\
& =-2 a i e^{-i \Delta t / 2} \sin \left(\Omega_{R} t / 2\right)
\end{aligned}
$$

In order to utilize the former initial condition $\left(c_{0}(0)=1\right)$, we need to take the derivative of Eq. 4.17, and plug this into Eq. 4.12 for $t=0$. Doing so yields $a=$ $-\Omega^{*} /\left(2 \Omega_{R}\right) e^{-i \delta_{o}}$. Therefore, the final solution for $c_{1}(t)$ is:

$$
c_{1}(t)=\frac{i \Omega^{*}}{\Omega_{R}} e^{-i \Delta t / 2} e^{-i \delta_{o}} \sin \left(\frac{1}{2} \Omega_{R} t\right)
$$




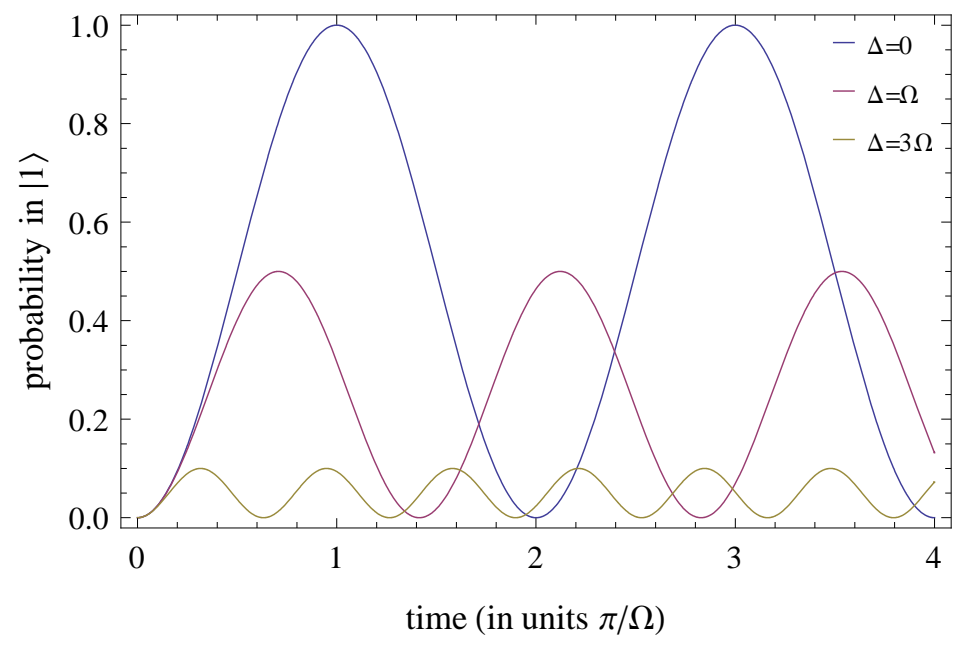

Figure 4.9: Theoretical Rabi oscillations given by Eq. 4.18 with detunings $\Delta=0, \Delta=\Omega$, and $\Delta=3 \Omega$.

The solution for $c_{0}(t)$ is obtained by simply plugging Eq. 4.18 into Eq. 4.12.

$$
c_{0}(t)=e^{i \Delta t / 2}\left[\cos \left(\frac{1}{2} \Omega_{R} t\right)-\frac{i \Delta}{\Omega_{R}} \sin \left(\frac{1}{2} \Omega_{R} t\right)\right]
$$

Figure 4.9 shows the theoretical $\left|c_{1}(t)\right|^{2}$ of Eq. 4.18 for a variety of detunings, $\Delta$.

If we had instead taken the initial conditions to be $c_{0}(0)=0$ and $c_{1}(0)=1$, the procedure for obtaining the final solution would have been the same, but the results would have been:

$$
\begin{aligned}
& c_{0}(t)=\frac{i \Omega}{\Omega_{R}} e^{i \Delta t / 2} e^{i \delta_{o}} \sin \left(\frac{1}{2} \Omega_{R} t\right) \\
& c_{1}(t)=e^{-i \Delta t / 2}\left[\cos \left(\frac{1}{2} \Omega_{R} t\right)+\frac{i \Delta}{\Omega_{R}} \sin \left(\frac{1}{2} \Omega_{R} t\right)\right]
\end{aligned}
$$

These two sets of solutions are all that we need to describe arbitrary microwave operations on the qubit. Any arbitrary initial state is simply a superposition of $|0\rangle$ and $|1\rangle$; e.g. $|\psi\rangle=\alpha|0\rangle+\beta|1\rangle$. The operation $R_{\mu W}$ acting on $|\psi\rangle$ is then $R_{\mu W}|\psi\rangle=\alpha R_{\mu W}|0\rangle+\beta R_{\mu W}|1\rangle$. Thus, if we know the action of $R_{\mu W}$ on $|0\rangle$ and $|1\rangle$, 
we know the action of $R_{\mu W}$ on any state. If we write the states as matrices

$$
|0\rangle=\left(\begin{array}{c}
1 \\
0
\end{array}\right) \text { and }|1\rangle=\left(\begin{array}{l}
0 \\
1
\end{array}\right)
$$

then the elements of the microwave operation matrix

$$
R_{\mu W}=\left(\begin{array}{cc}
a_{R} & b_{R} \\
c_{R} & d_{R}
\end{array}\right)
$$

have already been determined by our results above. Using Eq. 4.18 and 4.19, we find

$$
\begin{aligned}
R_{\mu W}|0\rangle & =\left(\begin{array}{cc}
a_{R} & b_{R} \\
c_{R} & d_{R}
\end{array}\right)\left(\begin{array}{c}
1 \\
0
\end{array}\right) \\
& =\left(\begin{array}{c}
a_{R} \\
c_{R}
\end{array}\right)=\left(\begin{array}{c}
e^{i \Delta t / 2}\left[\cos \left(\frac{1}{2} \Omega_{R} t\right)-\frac{i \Delta}{\Omega_{R}} \sin \left(\frac{1}{2} \Omega_{R} t\right)\right] \\
\frac{i \Omega^{*}}{\Omega_{R}} e^{-i \Delta t / 2} e^{-i \delta_{o}} \sin \left(\frac{1}{2} \Omega_{R} t\right)
\end{array}\right)
\end{aligned}
$$

Similarly, the other elements of the microwave operation matrix are determined by Eq. 4.20.

$$
\begin{aligned}
R_{\mu W}|1\rangle & =\left(\begin{array}{cc}
a_{R} & b_{R} \\
c_{R} & d_{R}
\end{array}\right)\left(\begin{array}{c}
0 \\
1
\end{array}\right) \\
& =\left(\begin{array}{c}
b_{R} \\
d_{R}
\end{array}\right)=\left(\begin{array}{c}
\frac{i \Omega}{\Omega_{R}} e^{i \Delta t / 2} e^{i \delta_{o}} \sin \left(\frac{1}{2} \Omega_{R} t\right) \\
e^{-i \Delta t / 2}\left[\cos \left(\frac{1}{2} \Omega_{R} t\right)+\frac{i \Delta}{\Omega_{R}} \sin \left(\frac{1}{2} \Omega_{R} t\right)\right]
\end{array}\right)
\end{aligned}
$$

Thus, we have defined operation of an arbitrary microwave pulse on an arbitrary qubit state by the matrix

$$
R_{\mu W}=\left(\begin{array}{cc}
e^{i \Delta t / 2}\left[\cos \left(\frac{1}{2} \Omega_{R} t\right)-\frac{i \Delta}{\Omega_{R}} \sin \left(\frac{1}{2} \Omega_{R} t\right)\right] & \frac{i \Omega^{*}}{\Omega_{R}} e^{-i \Delta t / 2} e^{-i \delta_{o}} \sin \left(\frac{1}{2} \Omega_{R} t\right) \\
\frac{i \Omega}{\Omega_{R}} e^{i \Delta t / 2} e^{i \delta_{o}} \sin \left(\frac{1}{2} \Omega_{R} t\right) & e^{-i \Delta t / 2}\left[\cos \left(\frac{1}{2} \Omega_{R} t\right)+\frac{i \Delta}{\Omega_{R}} \sin \left(\frac{1}{2} \Omega_{R} t\right)\right]
\end{array}\right)
$$

Generally, this kind of operation is termed a "rotation" of the qubit states. The terminology is a result of a geometric interpretation of the two-level system, where 
the two energy eigenstates $|0\rangle$ and $|1\rangle$ are positioned at either pole of the unit sphere (the Bloch sphere), and equal superposition states lie on the equator with the phase determining the azimuthal position.

With this geometric interpretation in mind, we define a couple of specific rotations that will be useful throughout the rest of this thesis. We define rotations with $\delta_{o}=3 \pi / 2$ as rotations about the $y$-axis of the Bloch sphere, and denote these by $R_{y}(\theta)$, where $\theta=\Omega_{R} t$ is the rotation angle. Rotations about the $x$-axis then correspond to $\delta_{o}=\pi$ and are written as $R_{x}(\theta)$. Of particular use will be the onresonance $(\Delta=0)$ " $\pi$-" and " $\pi / 2$ "-rotations, since this amounts to:

$$
\begin{aligned}
R_{y}(\pi)|0\rangle & =|1\rangle \\
R_{y}(\pi)|1\rangle & =-|0\rangle \\
R_{y}\left(\frac{\pi}{2}\right)|0\rangle & =\frac{1}{\sqrt{2}}(|0\rangle+|1\rangle) \\
R_{y}\left(\frac{\pi}{2}\right)|1\rangle & =\frac{1}{\sqrt{2}}(-|0\rangle+|1\rangle)
\end{aligned}
$$

and

$$
\begin{aligned}
R_{x}(\pi)|0\rangle & =-i|1\rangle \\
R_{x}(\pi)|1\rangle & =-i|0\rangle \\
R_{x}\left(\frac{\pi}{2}\right)|0\rangle & =\frac{1}{\sqrt{2}}(|0\rangle-i|1\rangle) \\
R_{x}\left(\frac{\pi}{2}\right)|1\rangle & =\frac{1}{\sqrt{2}}(-i|0\rangle+|1\rangle)
\end{aligned}
$$

In all of the forthcoming discussion of the experiments using these rotations, the applied microwave radiation will be near resonance. As such, in defining specific forms of rotations, we retain the approximation that $\Delta \ll \Omega$, so that $\Delta / \Omega_{R} \approx 0$ and $\Omega / \Omega_{R} \approx 1$. In addition, this means that during the typical time of a microwave operation $\left(\operatorname{order} \pi / \Omega\right.$ ) that the phase factor $e^{i \Delta t / 2} \approx 1$. However, if there is a long 
delay between two operations - such as in the Ramsey-type experiment explained below - then the detuning must be taken into account. In this case, the net effect of the detuning will be a rotation about the z-axis, as can be seen from Eq. 4.25 by setting $\Omega=0$.

The microwave rotations are implemented experimentally in one of two ways. Several watts of microwave power is either sent to a truncated waveguide that is $3 \mathrm{~cm}$ in length, located $7 \mathrm{~cm}$ from the center of the trap, or is applied directly to one of the trap rod electrodes. The choice of implementation is simply a matter of convenience; we have attempted both, with comparable results. The advantage of applying the microwave radiation directly to one of the electrodes of the trap is that, in principle, with the source closer to the atom the resulting Rabi frequencies could be much greater. In practice, though, the trap was not designed with this in mind, and so a lot of power is dissipated through parasitic capacitive couplings in the vacuum feedthrough. Also, when applying the microwave radiation directly on the trap the polarization is completely governed by the geometry of the structure, and is fixed. On the other hand, the truncated waveguide can be repositioned to optimize the coupling. Ultimately, these two methods resulted in approximately the same Rabi frequencies. A typical experimental Rabi oscillation between $|0\rangle$ and $|1\rangle$ in the ${ }^{171} \mathrm{Yb}^{+}$qubit is displayed in Fig. 4.10.

4.5.5 Coherence measurement We perform a Ramsey-type experiment to measure the coherence time of the qubit states, $|0\rangle$ and $|1\rangle$. In previous atomic clock work [35], a coherence time exceeding 15 minutes has been achieved with these same qubit levels. Since the coherence time of the qubit exceeds the stability of our microwave oscillator, we are unable to measure the coherence time of a single ion directly. Instead, we measure the coherence of one ion with respect to a second trapped 


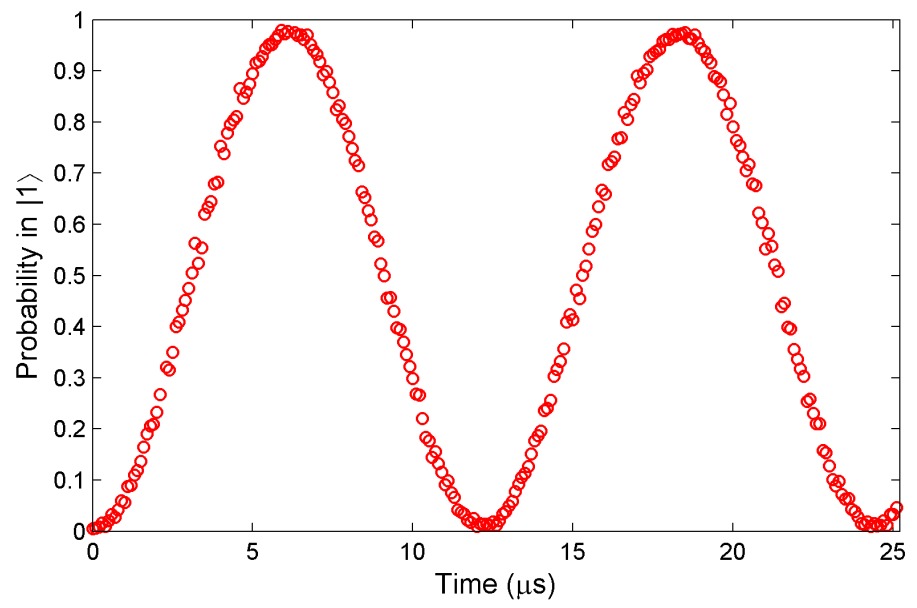

Figure 4.10: Rabi oscillations between the $|0\rangle$ and $|1\rangle$ states by application of microwaves at $12.642821 \mathrm{GHz}$. As seen in the figure, the time required to drive a $\pi$-pulse is about $6.0 \mu \mathrm{s}$. Each point is the result of 1000 measurements.

ion. The two ${ }^{171} \mathrm{Yb}^{+}$atoms are stored in two independent (nearly identical) traps separated by about one meter. A deliberate disparity in the magnetic field between the positions of the two ions alters the microwave transition frequency, which is $12.642821 \mathrm{GHz}$ at one ion position, by about $2.43 \mathrm{kHz}$ between the two ions. To measure the qubit coherence time, both ions are first initialized to $|0\rangle$. We then apply a microwave $\pi / 2$ pulse to the two ions, wait for a time $T / 2$, apply a microwave $\pi$ echo pulse to both ions, wait for another time $T / 2+\Delta t$, apply a microwave $\pi / 2$ analyzing pulse to both ions, and finally detect the state of each ion (see Fig. 4.11(a)). The phase of the second microwave $\pi / 2$ pulse is scrambled in order to eliminate the effect of the stability of the microwave oscillator. As a consequence, no Ramsey fringe is present in the signal from a single ion. However, a plot of the parity (the correlation between the measured states of the two ions) as a function of the additional delay $\Delta t$ displays a Ramsey fringe, as shown in Fig. 4.11(b). Lacking entanglement between the two ions, the amplitude of this fringe cannot exceed 0.5. As the delay time $T$ is increased, the amplitude of the Ramsey fringe decreases 
(Fig. 4.11(c)). Assuming gaussian fluctuations of the transition frequencies of the two ions, we fit the amplitude to a gaussian decay and obtain a 1/e coherence time of 2.5(3) seconds. The measured coherence time is likely limited by fluctuations of the differential magnetic field between the two ion positions through the second-order Zeeman shift. The coherence time should be significantly longer when lower static magnetic fields are used.

Thus, in this section we have demonstrated fast, efficient state initialization of the qubit through optical pumping; a state detection fidelity of $98.2(2) \%$ through statedependent fluorescence; reliable single-qubit operations using microwave radiation; and a qubit coherence time of $2.5(3)$ seconds. Overall, the ${ }^{171} \mathrm{Yb}^{+}$atom has been shown to be an excellent qubit candidate for applications in quantum information and quantum communication.

\section{6 $\quad{ }^{2} D_{3 / 2}$ and ${ }^{3}[3 / 2]_{1 / 2}$ hyperfine splittings}

We measured the hyperfine splitting of the ${ }^{2} D_{3 / 2}$ and ${ }^{3}[3 / 2]_{1 / 2}$ levels of ${ }^{171} \mathrm{Yb}^{+}$to determine the frequency needed to drive the ${ }^{2} D_{3 / 2}|F=2\rangle \leftrightarrow{ }^{3}[3 / 2]_{1 / 2}|F=1\rangle$ transition. For this measurement, the $935.2 \mathrm{~nm}$ laser was tuned far (about $3 \mathrm{GHz}$ ) from the ${ }^{2} D_{3 / 2}|F=1\rangle \leftrightarrow^{3}[3 / 2]_{1 / 2}|F=0\rangle$ transition, stabilized to the reference cavity, and the power is reduced to approximately $20 \mu \mathrm{W}$ at the trap. We then scanned the rf frequency applied to the fiber EOM in the $935.2 \mathrm{~nm}$ beam over a wide range (6 GHz). During this frequency scan, the fluorescence of the trapped ${ }^{171} \mathrm{Yb}^{+}$atom was monitored using the PMT. As one of the first-order sidebands generated by the fiber EOM scanned across either the ${ }^{2} D_{3 / 2}|F=1\rangle \leftrightarrow{ }^{3}[3 / 2]_{1 / 2}|F=0\rangle$ or the ${ }^{2} D_{3 / 2}|F=1\rangle \leftrightarrow{ }^{3}[3 / 2]_{1 / 2}|F=1\rangle$ resonance, many scattered $369.53 \mathrm{~nm}$ photons were observed. The hyperfine splitting of the ${ }^{3}[3 / 2]_{1 / 2}$ level is the frequency difference 
(a)
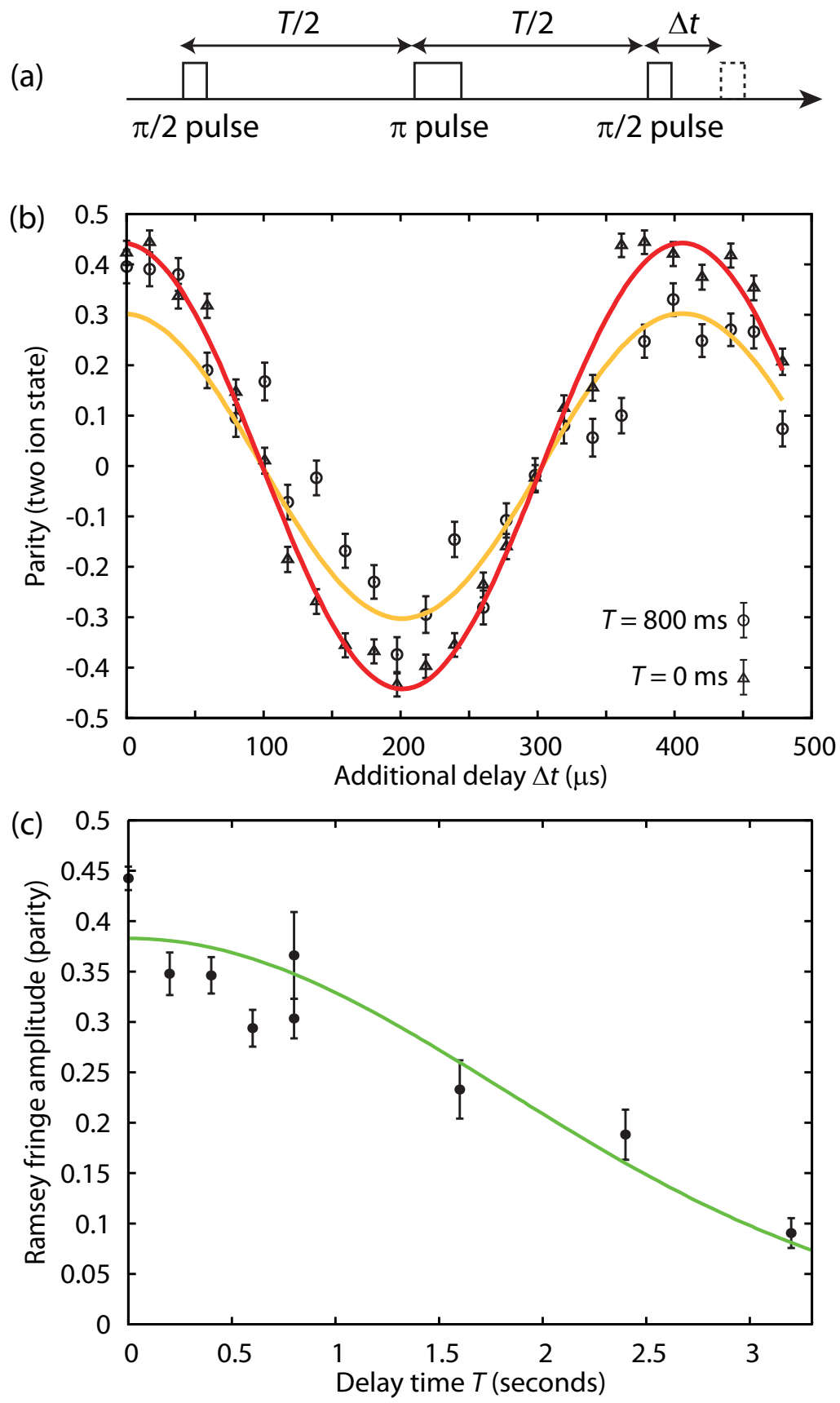

Figure 4.11: Measurement of the coherence time of the ${ }^{171} \mathrm{Yb}^{+}$qubit. (a) The series of microwave pulses used for this measurement. The two $\pi / 2$ pulses are separated by a variable time $T$, to measure the decay in the amplitude of the Ramsey fringe as a function of time. (b) Observed Ramsey fringes in the parity of the states of the two ions for delay times $T=0 \mathrm{~ms}$ and $T=800 \mathrm{~ms}$. (c) Decay in the amplitude of the observed Ramsey fringe as function of delay time $T$. The line is a gaussian fit to the data, with a $1 / \mathrm{e}$ decay time of $2.5(3)$ seconds. 
between these two resonances, and was measured to be 2.2095(11) GHz.

Next, we stabilized the laser at the ${ }^{2} D_{3 / 2}|F=1\rangle \leftrightarrow{ }^{3}[3 / 2]_{1 / 2}|F=0\rangle$ transition. While the ion was cooled by the $369.53 \mathrm{~nm}$ light, we also applied a train of $1 \mathrm{ps}$ pulses near $369.53 \mathrm{~nm}$, generated by frequency doubling the pulses from a modelocked Ti-sapphire laser. These broadband $(300 \mathrm{GHz})$ pulses couple to both of the hyperfine levels in ${ }^{2} S_{1 / 2}$. Thus, the ion is continuously excited to the ${ }^{2} P_{1 / 2}|F=1\rangle$ manifold, which eventually decays to ${ }^{2} D_{3 / 2}|F=2\rangle$, resulting in population trapping in this level. The first-order sidebands produced by the fiber EOM in the 935.2 nm beam are now scanned until one of the generated frequency components passes through resonance with the ${ }^{2} D_{3 / 2}|F=2\rangle \leftrightarrow^{3}[3 / 2]_{1 / 2}|F=1\rangle$ transition, signaled by an increased fluorescence of the ion. Given the hyperfine splitting of the ${ }^{3}[3 / 2]_{1 / 2}$ level stated above, we determined the hyperfine splitting of the ${ }^{2} D_{3 / 2}$ level to be $0.86(2) \mathrm{GHz}$. 


\section{CHAPTER V}

\section{Photon Interference}

(Alice asked,) "Would you tell me, please which way I ought to walk from here?"

"That depends a good deal on where you want to get to," said the Cat.

-Lewis Carroll, Alice's Adventures in Wonderland

The interference of identical single photons was first observed by Hong, Ou, and Mandel [108], and Shih and Alley [109]. In these experiments, pairs of photons created by parametric down-conversion were directed to interfere at a beamsplitter. It was observed that the interference of the photons at the beamsplitter resulted in two simultaneously impinging photons always exiting the beamsplitter by the same port. Thus, coincident detections behind the beamsplitter were highly suppressed. This antibunching of photons is now commonly used to charaterize experiments using single photons.

The quantum interference of two photons is an essential component of the quantum gate presented in this thesis. In this chapter, we present the theory for the interference of photons at a beamsplitter, and demonstrate the effect of this interference using single photons emitted by single trapped ions.

\subsection{Theoretical interference of photons at a beamsplitter}

We consider photons impinging on a 50:50, nonpolarizing beamsplitter, such as the one shown schematically in Fig. 5.1. We can describe photons and the effect of 


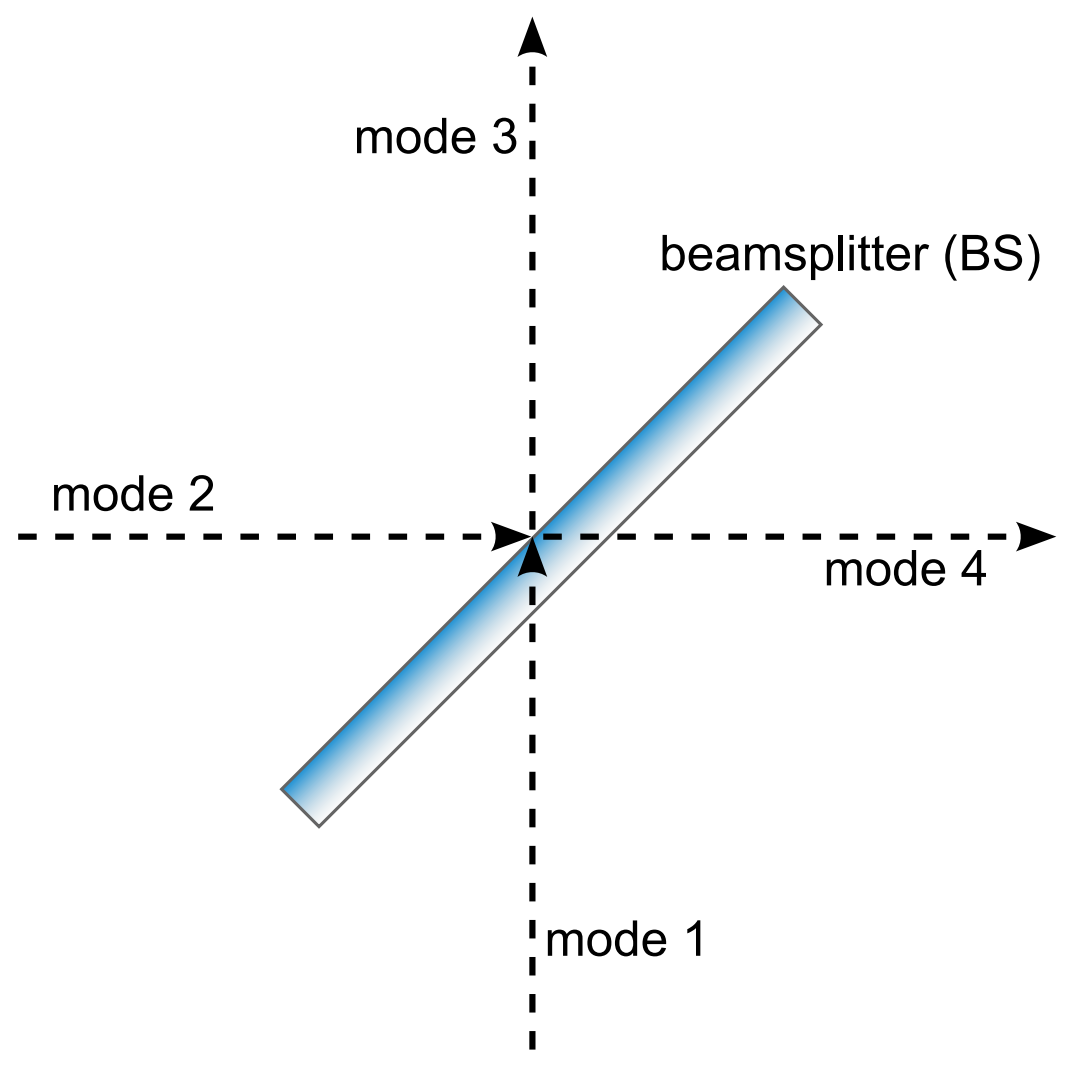

Figure 5.1: Schematic of a 50:50, nonpolarizing beamsplitter (BS). Modes 1 and 2 are the two input paths to the beamsplitter, while modes 3 and 4 are the exit paths from the beamsplitter. 
the beamsplitter using the usual photon creation operator $\hat{a}^{\dagger}$ and the vacuum state $|0\rangle$. A single photon in mode $n$ (as in Fig. 5.1) can than be written as $\left|1_{n}\right\rangle=\hat{a}_{n}^{\dagger}|0\rangle$. The operation of the beamsplitter is then encompassed by [110]:

$$
\begin{aligned}
& \hat{a}_{3}^{\dagger}=\frac{1}{\sqrt{2}}\left(\hat{a}_{1}^{\dagger}-\hat{a}_{2}^{\dagger}\right) \\
& \hat{a}_{4}^{\dagger}=\frac{1}{\sqrt{2}}\left(\hat{a}_{1}^{\dagger}+\hat{a}_{2}^{\dagger}\right)
\end{aligned}
$$

Of note is the sign difference between the decomposition of modes 3 and 4 in terms of modes 1 and 2 . The minus sign is the result of the $\pi$-phase shift experienced by light reflected at an interface where the index of refraction changes from low to high (e.g. [111], pg. 306). We can also rewrite Eq. 5.1 as

$$
\begin{aligned}
& \hat{a}_{1}^{\dagger}=\frac{1}{\sqrt{2}}\left(\hat{a}_{3}^{\dagger}+\hat{a}_{4}^{\dagger}\right) \\
& \hat{a}_{2}^{\dagger}=\frac{1}{\sqrt{2}}\left(-\hat{a}_{3}^{\dagger}+\hat{a}_{4}^{\dagger}\right)
\end{aligned}
$$

which is then effectively a decomposition of incoming photons in terms of the exiting photons.

The above formalism makes it easy to see the effect of the beamsplitter on impinging photons. We first consider the trivial case of a single photon in mode 1:

$$
\begin{aligned}
\left|1_{1}\right\rangle & =\hat{a}_{1}^{\dagger}|0\rangle \\
& =\frac{1}{\sqrt{2}}\left(\hat{a}_{3}^{\dagger}+\hat{a}_{4}^{\dagger}\right)|0\rangle \\
& =\frac{1}{\sqrt{2}}\left(\left|1_{3} 0_{4}\right\rangle+\left|0_{3} 1_{4}\right\rangle\right)
\end{aligned}
$$

As expected, we find that there is an equal (50\%) probability of detecting the photon in mode 3 as in mode $4 .{ }^{1}$ Of course, there is zero probability of getting a detection in both mode 3 and mode 4 simultaneously.

\footnotetext{
${ }^{1}$ On a sidenote, notice that Eq. 5.3 appears to indicate that the effect of the beamsplitter is to produce an entangled state, yet only one photon is involved. The remedy to this apparent paradox is to realize that the entanglement here is between two modes (mode 3 and mode 4) [112], although there is some controversy over this interpretation [113, 114].
} 
We next consider two photons impinging on the beamsplitter: one in mode 1 and one in mode 2. Following the same procedure as above, we then find:

$$
\begin{aligned}
\left|1_{1} 1_{2}\right\rangle & =\hat{a}_{1}^{\dagger} \hat{a}_{2}^{\dagger}|0\rangle \\
& =\frac{1}{2}\left(\hat{a}_{3}^{\dagger}+\hat{a}_{4}^{\dagger}\right)\left(-\hat{a}_{3}^{\dagger}+\hat{a}_{4}^{\dagger}\right)|0\rangle \\
& =\frac{1}{2}\left(\left(\hat{a}_{4}^{\dagger}\right)^{2}-\left(\hat{a}_{3}^{\dagger}\right)^{2}\right)|0\rangle \\
& =\frac{1}{\sqrt{2}}\left(\left|0_{3} 2_{4}\right\rangle-\left|2_{3} 0_{4}\right\rangle\right)
\end{aligned}
$$

and so we again find that there are no simultaneous detection of photons in modes 3 and 4. Instead, both photons either go into mode 3, or both photons go into mode 4, with equal probability. This simple analysis qualitatively explains the two-photon interference effect observed by Hong, Ou, and Mandel [108], and Shih and Alley [109].

Now let's generalize this formalism in a way that enables us to describe the ideal expected signal of our experiment; we shall follow the treatment presented in Ref. [110], but expand it to explicitly derive the signal expected from our experimental implementation. As described in the following section, the experiment to demonstrate this two-photon interference effect will consist of repetitive excitation of two trapped atomic ions, and detection of the spontaneously emitted photons behind a beamsplitter. If we assume that the repetitions of the experiment are wellseparated (as, indeed, is the design), then we can write the electric field operator of the mode $j$ for the $n^{\text {th }}$ repetition as

$$
E_{j}^{(n)+}(t)=\frac{A}{\sqrt{\tau}} \xi(r, t) e^{-\frac{1}{2}\left(t-n t_{p}\right) / \tau} \Theta\left(t-n t_{p}\right) \hat{a}_{j}^{(n)}
$$

where $t_{p}$ is the time between repetitions, $\tau$ is the natural lifetime of the excited state, and $\Theta$ is the Heaviside step function. The exponential decay factor accounts for the probability to detect a photon a given time interval following excitation. 
The $1 / \sqrt{\tau}$ factor ensures that the total probability of detecting a photon is unitless (integral over time of Eq. 5.8 below). The factor $A$ is an amplitude that depends on the collection efficiency of the optics, quantum efficiency of the detectors, etc. The function $\xi(r, t)$ describes the spatial mode of the photons, and can also be used to account for temporal offsets. For the remainder of this chapter, we shall ignore both $A$ and $\xi(r, t){ }^{2}$ The full electric field operator for the mode $j$ is then simply

$$
E_{j}^{+}(t)=\sum_{n} E_{j}^{(n)+}(t)
$$

The electric field operator describes the probability of detecting a photon at any given time.

The $N+1$ photons produced in the mode $j$ by $N+1$ repetitions of the experiment can be represented by the wavefunction

$$
\left|\psi_{N}\right\rangle_{j}=\left(\prod_{n=0}^{N} \hat{a}_{j}^{(n) \dagger}\right)|0\rangle
$$

where we have assumed that repetitions are sufficiently separated in time so that overlap of photons produced by different repetitions of the experiment is negligible; this allows us to write photons produced by different repetitions as separate "repetition modes" denoted by $n$ with the same spatial mode $j$.

If there are a train of photons being produced in mode $j$, then the probability of detecting a photon in mode $j$ at time $t$ is simply given by the first-order correlation

\footnotetext{
${ }^{2}$ We will find these factors to be useful later, when we calculate the error in the fidelity of the quantum gate and teleportation protocol due to mismatch of the photon spatial modes at the beamsplitter.
} 
function:

$$
\begin{aligned}
{ }_{j}\left\langle\psi_{N}\left|E_{j}^{-}(t) E_{j}^{+}(t)\right| \psi_{N}\right\rangle_{j}= & \langle 0|\left(\prod_{n=0}^{N} \hat{a}_{j}^{(n)}\right)\left(\sum_{k=0}^{N} \frac{1}{\sqrt{\tau}} e^{-\frac{1}{2}\left(t-k t_{p}\right) / \tau} \Theta\left(t-k t_{p}\right) \hat{a}_{j}^{(k) \dagger}\right) \\
& \times\left(\sum_{m=0}^{N} \frac{1}{\sqrt{\tau}} e^{-\frac{1}{2}\left(t-m t_{p}\right) / \tau} \Theta\left(t-m t_{p}\right) \hat{a}_{j}^{(m)}\right)\left(\prod_{n=0}^{N} \hat{a}_{j}^{(n) \dagger}\right)|0\rangle \\
= & \frac{1}{\tau} \sum_{k, m=0}^{N} e^{-\frac{1}{2}\left(t-k t_{p}\right) / \tau} e^{-\frac{1}{2}\left(t-m t_{p}\right) / \tau} \Theta\left(t-k t_{p}\right) \Theta\left(t-m t_{p}\right) \\
& \times\left\langle 0\left|\left(\prod_{n=0}^{N} \hat{a}_{j}^{(n)}\right) \hat{a}_{j}^{(k) \dagger} \hat{a}_{j}^{(m)}\left(\prod_{n=0}^{N} \hat{a}_{j}^{(n) \dagger}\right)\right| 0\right\rangle \\
= & \frac{1}{\tau} \sum_{k, m=0}^{N} e^{-\frac{1}{2}\left(t-k t_{p}\right) / \tau} e^{-\frac{1}{2}\left(t-m t_{p}\right) / \tau} \Theta\left(t-k t_{p}\right) \Theta\left(t-m t_{p}\right) \delta_{k m} \\
= & \frac{1}{\tau} \sum_{k=0}^{N} e^{-\left(t-k t_{p}\right) / \tau} \Theta\left(t-k t_{p}\right)
\end{aligned}
$$

where, as mentioned above, we have ignored both $A$ and $\xi(r, t)$ from Eq. 5.5. Figure 5.2 illustrates this result for the case $N=10$. As shown in the figure, the probability of detecting a photon from the $n^{\text {th }}$ repetition of the experiment decays exponentially with decay constant given by the natural lifetime of the excited state $(\tau)$. The probability for a joint detection in both mode 3 and mode 4 , given a train of photons being produced in mode 1 , is given by the second-order correlation function:

$$
\begin{aligned}
P_{J}\left(t, t_{d}\right)= & { }_{1}\left\langle\psi_{N}\left|E_{3}^{-}(t) E_{4}^{-}\left(t+t_{d}\right) E_{4}^{+}\left(t+t_{d}\right) E_{3}^{+}(t)\right| \psi_{N}\right\rangle_{1} \\
= & \langle 0|\left(\prod_{m=0}^{N} \hat{a}_{1}^{(m)}\right)\left(\sum_{k=0}^{N} \frac{1}{\sqrt{\tau}} e^{-\frac{1}{2}\left(t-k t_{p}\right) / \tau} \Theta\left(t-k t_{p}\right) \hat{a}_{3}^{(k) \dagger}\right) \\
& \times\left(\sum_{n=0}^{N} \frac{1}{\sqrt{\tau}} e^{-\frac{1}{2}\left(t+t_{d}-n t_{p}\right) / \tau} \Theta\left(t+t_{d}-n t_{p}\right) \hat{a}_{4}^{(n) \dagger}\right) \\
& \times\left(\sum_{n=0}^{N} \frac{1}{\sqrt{\tau}} e^{-\frac{1}{2}\left(t+t_{d}-n t_{p}\right) / \tau} \Theta\left(t+t_{d}-n t_{p}\right) \hat{a}_{4}^{(n)}\right) \\
& \times\left(\sum_{k=0}^{N} \frac{1}{\sqrt{\tau}} e^{-\frac{1}{2}\left(t-k t_{p}\right) / \tau} \Theta\left(t-k t_{p}\right) \hat{a}_{3}^{(k)}\right)\left(\prod_{m=0}^{N} \hat{a}_{1}^{(m) \dagger}\right)|0\rangle
\end{aligned}
$$

Notice that since in order to be non-zero the two halves of the above equation need 


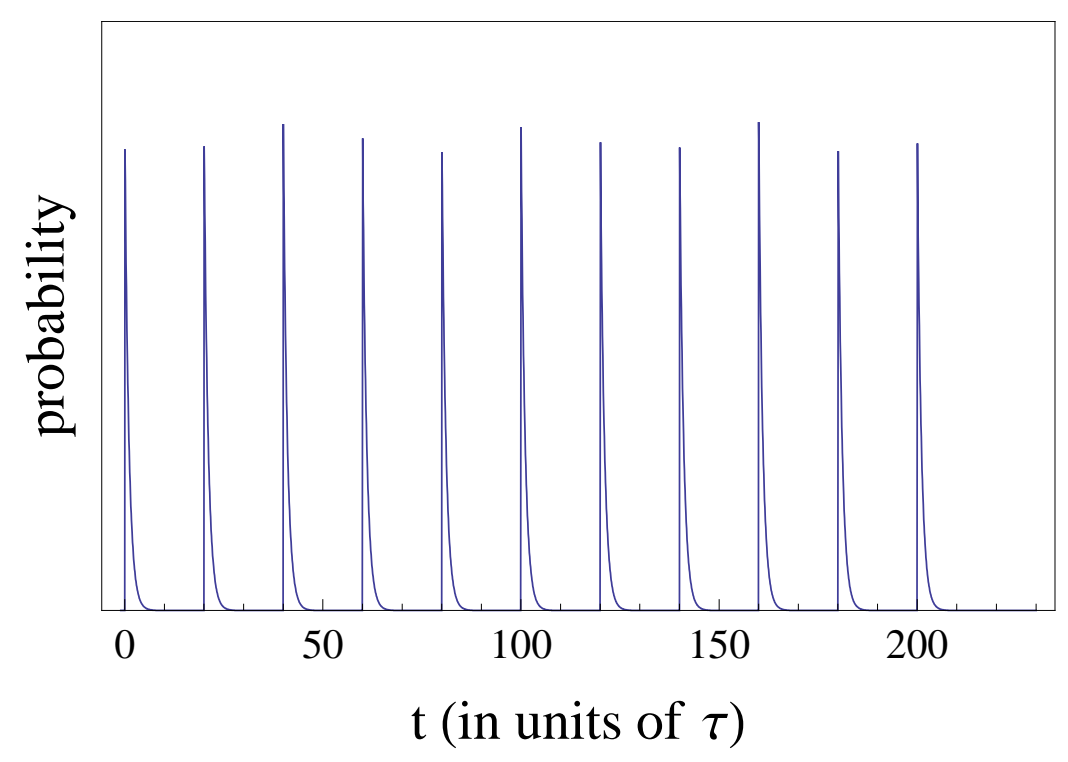

Figure 5.2: Theoretical single photon first-order correlation function, given by Eq. 5.8. This shows the relative probability of detecting a photon in mode $j$ at time $t$ following a series of 10 excitations. The charateristic exponential decay after each repetition/excitation in the experiment is determined by the natural lifetime of the excited state of the atom producing the spontaneously emitted photons. The repetition rate shown is about 20 times the natural lifetime $(\tau)$.

to be hermitian conjugates of each other, we can use the same summation index for both the positive and negative components of the electric field operator of a given mode; this is just to say that we need the same number of creation/annihilation operators acting on the 'bra'/'ket' for the term not to vanish. We can decompose the creation and annhilation operators for modes 3 and 4 in terms of modes 1 and 2, using Eq. 5.1. Of course, since in the above equation there are no photons in mode 2 , it is clear that any term with an annihilation operator of mode 2 will immediately 
vanish. This allows us to simpifly Eq. 5.9 to obtain

$$
\begin{aligned}
P_{J}\left(t, t_{d}\right)= & \frac{1}{4 \tau^{2}}\langle 0|\left(\prod_{m=0}^{N} \hat{a}_{1}^{(m)}\right)\left(\sum_{k=0}^{N} e^{-\frac{1}{2}\left(t-k t_{p}\right) / \tau} \Theta\left(t-k t_{p}\right) \hat{a}_{1}^{(k) \dagger}\right) \\
& \times\left(\sum_{n=0}^{N} e^{-\frac{1}{2}\left(t+t_{d}-n t_{p}\right) / \tau} \Theta\left(t+t_{d}-n t_{p}\right) \hat{a}_{1}^{(n) \dagger}\right) \\
& \times\left(\sum_{n=0}^{N} e^{-\frac{1}{2}\left(t+t_{d}-n t_{p}\right) / \tau} \Theta\left(t+t_{d}-n t_{p}\right) \hat{a}_{1}^{(n)}\right) \\
& \times\left(\sum_{k=0}^{N} e^{-\frac{1}{2}\left(t-k t_{p}\right) / \tau} \Theta\left(t-k t_{p}\right) \hat{a}_{1}^{(k)}\right)\left(\prod_{m=0}^{N} \hat{a}_{1}^{(m) \dagger}\right)|0\rangle \\
= & \frac{1}{4 \tau^{2}} \sum_{k=0}^{N} \sum_{n=0}^{N} e^{-\left(t-k t_{p}\right) / \tau} e^{-\left(t+t_{d}-n t_{p}\right) / \tau} \Theta\left(t-k t_{p}\right) \Theta\left(t+t_{d}-n t_{p}\right) \\
& \times\left\langle 0\left|\left(\prod_{m=0}^{N} \hat{a}_{1}^{(m)}\right) \hat{a}_{1}^{(k) \dagger} \hat{a}_{1}^{(n) \dagger} \hat{a}_{1}^{(n)} \hat{a}_{1}^{(k)}\left(\prod_{m=0}^{N} \hat{a}_{1}^{(m) \dagger}\right)\right| 0\right\rangle \\
= & \frac{1}{4 \tau^{2}} \sum_{k=0}^{N} \sum_{n=0}^{N} e^{-\left(t-k t_{p}\right) / \tau} e^{-\left(t+t_{d}-n t_{p}\right) / \tau} \Theta\left(t-k t_{p}\right) \Theta\left(t+t_{d}-n t_{p}\right) \\
& \times\left(1-\delta_{k n}\right)
\end{aligned}
$$

where the last step is simply a consequence of having an annihilation operator for both $k$ and $n$, but only one creation operator for $m$; thus, if $k=n$ the term goes to zero. Of course, we are interested in the total probability of a joint detection as a function of the time delay $t_{d}$ between the two photons. In order to obtain this 
function, we need to integrate Eq. 5.10 over $t$.

$$
\begin{aligned}
P_{\text {Jtot }}\left(t_{d}\right)= & \int_{-\infty}^{\infty} P_{\text {joint }}\left(t, t_{d}\right) \mathrm{d} t \\
= & \frac{1}{4 \tau^{2}} \sum_{k=0}^{N} \sum_{n=0}^{N}\left(1-\delta_{k n}\right) \\
& \times \int_{-\infty}^{\infty} e^{-\left(t-k t_{p}\right) / \tau} e^{-\left(t+t_{d}-n t_{p}\right) / \tau} \Theta\left(t-k t_{p}\right) \Theta\left(t+t_{d}-n t_{p}\right) \mathrm{d} t \\
= & \frac{1}{4 \tau^{2}} \sum_{k=0}^{N} \sum_{n=0}^{N}\left(1-\delta_{k n}\right) e^{-\left(t_{d}-t_{p}(k+n)\right) / \tau} \\
& \times \int_{-\infty}^{\infty} e^{-2 t / \tau} \Theta\left(t-k t_{p}\right) \Theta\left(t+t_{d}-n t_{p}\right) \mathrm{d} t \\
= & \frac{1}{4 \tau^{2}} \sum_{k=0}^{N} \sum_{n=0}^{N}\left(1-\delta_{k n}\right) e^{-\left(t_{d}-t_{p}(k+n)\right) / \tau} \\
& \times \int_{k t_{p}}^{\infty} e^{-2 t / \tau} \Theta\left(t+t_{d}-n t_{p}\right) \mathrm{d} t \\
= & \frac{1}{4 \tau^{2}} \sum_{k=0}^{N} \sum_{n=0}^{N}\left(1-\delta_{k n}\right) e^{\left(t_{d}+t_{p}(k-n)\right) / \tau} \\
& \times \int_{t_{d}+t_{p}(k-n)}^{\infty} e^{-2 u / \tau} \Theta(u) \mathrm{d} u
\end{aligned}
$$

where in the last line we've set $u=t+t_{d}-n t_{p}$. The integration is completed by looking at two cases:

$$
\begin{aligned}
P_{\text {Jtot }}\left(t_{d} \leq-t_{p}(k-n)\right)= & \frac{1}{4 \tau^{2}} \sum_{k=0}^{N} \sum_{n=0}^{N}\left(1-\delta_{k n}\right) e^{\left(t_{d}+t_{p}(k-n)\right) / \tau} \\
& \times \int_{0}^{\infty} e^{-2 u / \tau} \Theta(u) \mathrm{d} u \\
= & \frac{1}{4 \tau^{2}} \sum_{k=0}^{N} \sum_{n=0}^{N}\left(1-\delta_{k n}\right) e^{\left(t_{d}+t_{p}(k-n)\right) / \tau} \\
& \times\left[-\frac{\tau}{2} e^{-2 u / \tau}\right]_{0}^{\infty} \\
= & \frac{1}{8 \tau} \sum_{k=0}^{N} \sum_{n=0}^{N}\left(1-\delta_{k n}\right) e^{\left(t_{d}+t_{p}(k-n)\right) / \tau}
\end{aligned}
$$




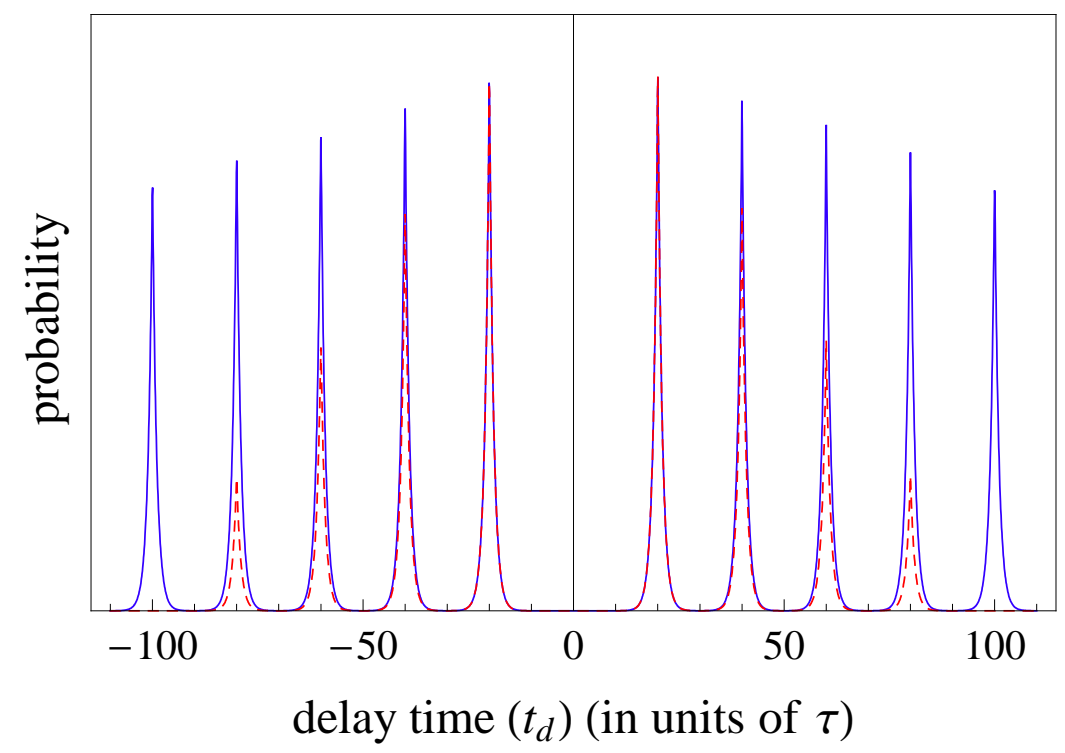

Figure 5.3: Theoretical single photon second-order correlation function, given by Eq. 5.14. This shows the relative probability of detecting a photon in both mode 3 and mode 4 as a function of the time delay $\left(t_{d}\right)$ between the two photons for $N=4$ (red, dashed) and $N=20$ (blue, solid). The repetition rate shown is about 20 times the natural lifetime of the atomic excited state $(\tau)$. The lack of a peak at delay time $t_{d}=0$ is characteristic of a single-photon source.

and

$$
\begin{aligned}
P_{J t o t}\left(t_{d} \geq-t_{p}(k-n)\right)= & \frac{1}{4 \tau^{2}} \sum_{k=0}^{N} \sum_{n=0}^{N}\left(1-\delta_{k n}\right) e^{\left(t_{d}+t_{p}(k-n)\right) / \tau} \\
& \times \int_{t_{d}+t_{p}(k-n)}^{\infty} e^{-2 u / \tau} \Theta(u) \mathrm{d} u \\
= & \frac{1}{4 \tau^{2}} \sum_{k=0}^{N} \sum_{n=0}^{N}\left(1-\delta_{k n}\right) e^{\left(t_{d}+t_{p}(k-n)\right) / \tau} \\
& \times\left[-\frac{\tau}{2} e^{-2 u / \tau}\right]_{t_{d}+t_{p}(k-n)}^{\infty} \\
= & \frac{1}{8 \tau} \sum_{k=0}^{N} \sum_{n=0}^{N}\left(1-\delta_{k n}\right) e^{-\left(t_{d}+t_{p}(k-n)\right) / \tau}
\end{aligned}
$$

By inspection it is then clear that Eq. 5.12 and 5.13 can be written succinctly as:

$$
P_{J t o t}\left(t_{d}\right)=\frac{1}{8 \tau} \sum_{k=0}^{N} \sum_{n=0}^{N}\left(1-\delta_{k n}\right) e^{-\left|t_{d}+t_{p}(k-n)\right| / \tau}
$$

This result is plotted in Fig. 5.3. As expected from Eq. 5.3, the probability of simultaneously detecting a photon in both mode 3 and mode 4 is zero, as indicated 
by the lack of a peak at delay time $t_{d}=0$. Experimentally, the number of detection events at $t_{d}=0$ relative to the height of the adjacent peaks can be used to evaluate the fidelity of single-photon generation $[115,69]$.

We now look at the signal we would expect from photons impinging on both input ports of the beamsplitter. We use Eq. 5.7 to express the photonic wavefunction for a train of pairs of photons in modes 1 and 2 as:

$$
\begin{aligned}
\left|\psi_{N}\right\rangle_{1,2} & =\left(\prod_{n=0}^{N} \hat{a}_{1}^{(n) \dagger}\right)\left(\prod_{m=0}^{N} \hat{a}_{2}^{(m) \dagger}\right)|0\rangle \\
& =\left(\prod_{n=0}^{N} \hat{a}_{1}^{(n) \dagger} \hat{a}_{2}^{(n) \dagger}\right)|0\rangle
\end{aligned}
$$

where the second line follows from the fact that $\left[\hat{a}_{1}^{(n) \dagger}, \hat{a}_{2}^{(m) \dagger}\right]=0 \forall m, n$. Here we assume that the "repetition modes" are common to the two pulse trains; in other words, we assume to have pairs of identical photons impinging on the beamsplitter. ${ }^{3}$

The second-order correlation function for pairs of photons can then be calculated in a way similar to Eq. 5.9.

$$
\begin{aligned}
P_{2 p h, J}\left(t, t_{d}\right)= & { }_{1,2}\left\langle\psi_{N}\left|E_{3}^{-}(t) E_{4}^{-}\left(t+t_{d}\right) E_{4}^{+}\left(t+t_{d}\right) E_{3}^{+}(t)\right| \psi_{N}\right\rangle_{1,2} \\
= & \langle 0|\left(\prod_{m=0}^{N} \hat{a}_{1}^{(m)} \hat{a}_{2}^{(m)}\right)\left(\sum_{k=0}^{N} \frac{1}{\sqrt{\tau}} e^{-\frac{1}{2}\left(t-k t_{p}\right) / \tau} \Theta\left(t-k t_{p}\right) \hat{a}_{3}^{(k) \dagger}\right) \\
& \times\left(\sum_{n=0}^{N} \frac{1}{\sqrt{\tau}} e^{-\frac{1}{2}\left(t+t_{d}-n t_{p}\right) / \tau} \Theta\left(t+t_{d}-n t_{p}\right) \hat{a}_{4}^{(n) \dagger}\right) \\
& \times\left(\sum_{n=0}^{N} \frac{1}{\sqrt{\tau}} e^{-\frac{1}{2}\left(t+t_{d}-n t_{p}\right) / \tau} \Theta\left(t+t_{d}-n t_{p}\right) \hat{a}_{4}^{(n)}\right) \\
& \times\left(\sum_{k=0}^{N} \frac{1}{\sqrt{\tau}} e^{-\frac{1}{2}\left(t-k t_{p}\right) / \tau} \Theta\left(t-k t_{p}\right) \hat{a}_{3}^{(k)}\right) \\
& \times\left(\prod_{m=0}^{N} \hat{a}_{1}^{(m) \dagger} \hat{a}_{2}^{(m) \dagger}\right)|0\rangle
\end{aligned}
$$

\footnotetext{
${ }^{3}$ The case of non-overlapping temporal modes 1 and 2 is a trivial extension of the single-photon result above. Slight temporal mismatch between photons in modes 1 and 2 would need to be accounted for in the definition of the electric field operator, Eq. 5.5. Since in the present section we are interested in deriving the ideal signal that would be measured by this kind of photon interference experiment, we assume the ideal case of photons from the same repetition having perfect temporal overlap.
} 
Of course, the time dependence of Eq. 5.16 appears identical to Eq. 5.10. Thus, the integration over time will be identical to the single-photon case, and so for the present calculation we only need to focus on the effect of the additional creation and annihilation operators. Writing the operators for modes 3 and 4 in terms of modes 1 and 2 yields:

$$
\begin{aligned}
P_{2 p h, J}\left(t, t_{d}\right) \propto & \left\langle 0\left|\left(\prod_{m=0}^{N} \hat{a}_{1}^{(m)} \hat{a}_{2}^{(m)}\right) \hat{a}_{3}^{(k) \dagger} \hat{a}_{4}^{(n) \dagger} \hat{a}_{4}^{(n)} \hat{a}_{3}^{(k)}\left(\prod_{m=0}^{N} \hat{a}_{1}^{(m) \dagger} \hat{a}_{2}^{(m) \dagger}\right)\right| 0\right\rangle \\
= & \frac{1}{4}\langle 0|\left(\prod_{m=0}^{N} \hat{a}_{1}^{(m)} \hat{a}_{2}^{(m)}\right)\left(\hat{a}_{1}^{(k) \dagger}-\hat{a}_{2}^{(k) \dagger}\right)\left(\hat{a}_{1}^{(n) \dagger}+\hat{a}_{2}^{(n) \dagger}\right) \\
& \times\left(\hat{a}_{1}^{(n)}+\hat{a}_{2}^{(n)}\right)\left(\hat{a}_{1}^{(k)}-\hat{a}_{2}^{(k)}\right)\left(\prod_{m=0}^{N} \hat{a}_{1}^{(m) \dagger} \hat{a}_{2}^{(m) \dagger}\right)|0\rangle \\
= & \frac{1}{4}\langle 0|\left(\prod_{m=0}^{N} \hat{a}_{1}^{(m)} \hat{a}_{2}^{(m)}\right)\left(\hat{a}_{1}^{(k) \dagger} \hat{a}_{1}^{(n) \dagger} \hat{a}_{1}^{(n)} \hat{a}_{1}^{(k)}+\hat{a}_{1}^{(k) \dagger} \hat{a}_{1}^{(n) \dagger} \hat{a}_{2}^{(n)} \hat{a}_{1}^{(k)}\right. \\
& -\hat{a}_{1}^{(k) \dagger} \hat{a}_{1}^{(n) \dagger} \hat{a}_{1}^{(n)} \hat{a}_{2}^{(k)}-\hat{a}_{1}^{(k) \dagger} \hat{a}_{1}^{(n) \dagger} \hat{a}_{2}^{(n)} \hat{a}_{2}^{(k)}-\hat{a}_{2}^{(k) \dagger} \hat{a}_{1}^{(n) \dagger} \hat{a}_{1}^{(n)} \hat{a}_{1}^{(k)} \\
& -\hat{a}_{2}^{(k) \dagger} \hat{a}_{1}^{(n) \dagger} \hat{a}_{2}^{(n)} \hat{a}_{1}^{(k)}+\hat{a}_{2}^{(k) \dagger} \hat{a}_{1}^{(n) \dagger} \hat{a}_{1}^{(n)} \hat{a}_{2}^{(k)}+\hat{a}_{2}^{(k) \dagger} \hat{a}_{1}^{(n) \dagger} \hat{a}_{2}^{(n)} \hat{a}_{2}^{(k)} \\
& +\hat{a}_{1}^{(k) \dagger} \hat{a}_{2}^{(n) \dagger} \hat{a}_{1}^{(n)} \hat{a}_{1}^{(k)}+\hat{a}_{1}^{(k) \dagger} \hat{a}_{2}^{(n) \dagger} \hat{a}_{2}^{(n)} \hat{a}_{1}^{(k)}-\hat{a}_{1}^{(k) \dagger} \hat{a}_{2}^{(n) \dagger} \hat{a}_{1}^{(n)} \hat{a}_{2}^{(k)} \\
& -\hat{a}_{1}^{(k) \dagger} \hat{a}_{2}^{(n) \dagger} \hat{a}_{2}^{(n)} \hat{a}_{2}^{(k)}-\hat{a}_{2}^{(k) \dagger} \hat{a}_{2}^{(n) \dagger} \hat{a}_{1}^{(n)} \hat{a}_{1}^{(k)}-\hat{a}_{2}^{(k) \dagger} \hat{a}_{2}^{(n) \dagger} \hat{a}_{2}^{(n)} \hat{a}_{1}^{(k)} \\
& \left.+\hat{a}_{2}^{(k) \dagger} \hat{a}_{2}^{(n) \dagger} \hat{a}_{1}^{(n)} \hat{a}_{2}^{(k)}+\hat{a}_{2}^{(k) \dagger} \hat{a}_{2}^{(n) \dagger} \hat{a}_{2}^{(n)} \hat{a}_{2}^{(k)}\right)\left(\prod_{m}^{N} \hat{a}_{1}^{(m) \dagger} \hat{a}_{2}^{(m) \dagger}\right)|0\rangle
\end{aligned}
$$

Only the terms with an equal number of creation/annihilation operators for each mode with be nonzero. Eliminating the unbalanced terms yields:

$$
\begin{aligned}
P_{2 p h, J}\left(t, t_{d}\right) \propto & \frac{1}{4}\langle 0|\left(\prod_{m=0}^{N} \hat{a}_{1}^{(m)} \hat{a}_{2}^{(m)}\right)\left(\hat{a}_{1}^{(k) \dagger} \hat{a}_{1}^{(n) \dagger} \hat{a}_{1}^{(n)} \hat{a}_{1}^{(k)}-\hat{a}_{2}^{(k) \dagger} \hat{a}_{1}^{(n) \dagger} \hat{a}_{2}^{(n)} \hat{a}_{1}^{(k)}\right. \\
& +\hat{a}_{2}^{(k) \dagger} \hat{a}_{1}^{(n) \dagger} \hat{a}_{1}^{(n)} \hat{a}_{2}^{(k)}+\hat{a}_{1}^{(k) \dagger} \hat{a}_{2}^{(n) \dagger} \hat{a}_{2}^{(n)} \hat{a}_{1}^{(k)}-\hat{a}_{1}^{(k) \dagger} \hat{a}_{2}^{(n) \dagger} \hat{a}_{1}^{(n)} \hat{a}_{2}^{(k)} \\
& \left.+\hat{a}_{2}^{(k) \dagger} \hat{a}_{2}^{(n) \dagger} \hat{a}_{2}^{(n)} \hat{a}_{2}^{(k)}\right)\left(\prod_{m=0}^{N} \hat{a}_{1}^{(m) \dagger} \hat{a}_{2}^{(m) \dagger}\right)|0\rangle \\
= & \left(1-\delta_{k n}\right)-\delta_{k n}+1+1-\delta_{k n}+\left(1-\delta_{k n}\right) \\
= & 4\left(1-\delta_{k n}\right)
\end{aligned}
$$


The final solution, after integrating over $t$ (with integrals identical to Eq. 5.11), is

$$
P_{2 p h, J t o t}\left(t_{d}\right)=\frac{1}{2 \tau} \sum_{k=0}^{N} \sum_{n=0}^{N}\left(1-\delta_{k n}\right) e^{-\left|t_{d}+t_{p}(k-n)\right| / \tau}
$$

which is just Eq. 5.14 multiplied by a factor of 4 . This indicates that there is zero probability of obtaining simultaneous dectections in both modes 3 and 4 if the incoming photons in modes 1 and 2 are identical, as was determined qualitatively in Eq. 5.4. The additional factor of 4 results because in this case one photon in mode 3 and one photon in mode 4 separated by non-zero delay time $t_{d}$ can be the result of: 2 sequential photons in mode $1 ; 2$ sequential photons in mode $2 ; 1$ photon in mode 1 followed by 1 photon in mode 2; 1 photon in mode 2 followed by 1 photon in mode 1.

It might be assumed that the fidelity of a two-photon interference experiment can then be evaluated by taking the ratio of the number of detection events at $t_{d}=0$ to the number detected at a multiple of the experiment repetition rate $t_{d}=n t_{p}$. As it turns out, this is the right approach, but would overestimate the fidelity by a factor of 2. In order to understand why, we need to calculate the second-order correlation function for an incoming train of distinguishable photons. If we assume the incoming photons in mode 1 can be distinguished from those in mode 2 (for instance, by polarization), then we can rewrite the two-photon wavefunction from Eq. 5.15 as:

$$
\left|\psi_{N}\right\rangle_{1 a, 2 b}=\left(\prod_{n=0}^{N} \hat{a}_{1}^{(n) \dagger} \hat{b}_{2}^{(n) \dagger}\right)|0\rangle
$$

with $\left[\hat{a}_{i}^{\dagger}, \hat{b}_{j}\right]=0 \forall i, j$. We can then calculate the second-order correlation function. However, we need to be careful to allow the four possible (distinguishable) cases: detecting an $\hat{a}^{\dagger}$ photon in mode 3 and a $\hat{b}^{\dagger}$ photon in mode 4 ; detecting a $\hat{b}^{\dagger}$ photon in mode 3 and an $\hat{a}^{\dagger}$ photon in mode 4; detecting an $\hat{a}^{\dagger}$ photon in both mode 3 and 
mode 4 ; and detecting a $\hat{b}^{\dagger}$ photon in both mode 3 and mode 4 . Thus,

$$
\begin{aligned}
P_{a, b, J}\left(t, t_{d}\right)= & { }_{1 a, 2 b}\left\langle\psi_{N}\left|E_{3 a}^{-}(t) E_{4 b}^{-}\left(t+t_{d}\right) E_{4 b}^{+}\left(t+t_{d}\right) E_{3 a}^{+}(t)\right| \psi_{N}\right\rangle_{1 a, 2 b} \\
& +{ }_{1 a, 2 b}\left\langle\psi_{N}\left|E_{3 b}^{-}(t) E_{4 a}^{-}\left(t+t_{d}\right) E_{4 a}^{+}\left(t+t_{d}\right) E_{3 b}^{+}(t)\right| \psi_{N}\right\rangle_{1 a, 2 b} \\
& +{ }_{1 a, 2 b}\left\langle\psi_{N}\left|E_{3 a}^{-}(t) E_{4 a}^{-}\left(t+t_{d}\right) E_{4 a}^{+}\left(t+t_{d}\right) E_{3 a}^{+}(t)\right| \psi_{N}\right\rangle_{1 a, 2 b} \\
& +{ }_{1 a, 2 b}\left\langle\psi_{N}\left|E_{3 b}^{-}(t) E_{4 b}^{-}\left(t+t_{d}\right) E_{4 b}^{+}\left(t+t_{d}\right) E_{3 b}^{+}(t)\right| \psi_{N}\right\rangle_{1 a, 2 b} \\
= & 2_{1 a, 2 b}\left\langle\psi_{N}\left|E_{3 a}^{-}(t) E_{4 b}^{-}\left(t+t_{d}\right) E_{4 b}^{+}\left(t+t_{d}\right) E_{3 a}^{+}(t)\right| \psi_{N}\right\rangle_{1 a, 2 b} \\
& +2_{1 a, 2 b}\left\langle\psi_{N}\left|E_{3 a}^{-}(t) E_{4 a}^{-}\left(t+t_{d}\right) E_{4 a}^{+}\left(t+t_{d}\right) E_{3 a}^{+}(t)\right| \psi_{N}\right\rangle_{1 a, 2 b}
\end{aligned}
$$

where the last line follows from symmetry. In addition, since $\left[\hat{a}_{i}^{\dagger}, \hat{b}_{j}\right]=0 \forall i, j$, the second term in Eq. 5.20 is equal to (two times) the second-order correlation function for a train of single-photons (Eq. 5.9). As we can just quote the value found above for this term, now we just need to calculate the first term.

$$
\begin{aligned}
& 2_{1 a, 2 b}\left\langle\psi_{N}\left|E_{3 a}^{-}(t) E_{4 b}^{-}\left(t+t_{d}\right) E_{4 b}^{+}\left(t+t_{d}\right) E_{3 a}^{+}(t)\right| \psi_{N}\right\rangle_{1 a, 2 b} \\
= & 2\langle 0|\left(\prod_{m=0}^{N} \hat{a}_{1}^{(m)} \hat{b}_{2}^{(m)}\right)\left(\sum_{k=0}^{N} \frac{1}{\sqrt{\tau}} e^{-\frac{1}{2}\left(t-k t_{p}\right) / \tau} \Theta\left(t-k t_{p}\right) \hat{a}_{3}^{(k) \dagger}\right) \\
& \times\left(\sum_{n=0}^{N} \frac{1}{\sqrt{\tau}} e^{-\frac{1}{2}\left(t+t_{d}-n t_{p}\right) / \tau} \Theta\left(t+t_{d}-n t_{p}\right) \hat{b}_{4}^{(n) \dagger}\right) \\
& \times\left(\sum_{n=0}^{N} \frac{1}{\sqrt{\tau}} e^{-\frac{1}{2}\left(t+t_{d}-n t_{p}\right) / \tau} \Theta\left(t+t_{d}-n t_{p}\right) \hat{b}_{4}^{(n)}\right) \\
& \times\left(\sum_{k=0}^{N} \frac{1}{\sqrt{\tau}} e^{-\frac{1}{2}\left(t-k t_{p}\right) / \tau} \Theta\left(t-k t_{p}\right) \hat{a}_{3}^{(k)}\right)\left(\prod_{m=0}^{N} \hat{a}_{1}^{(m) \dagger} \hat{b}_{2}^{(m) \dagger}\right)|0\rangle \\
= & \frac{2}{\tau^{2}} \sum_{k=0}^{N} \sum_{n=0}^{N} e^{-\left(t-k t_{p}\right) / \tau} e^{-\left(t+t_{d}-n t_{p}\right) / \tau} \Theta\left(t-k t_{p}\right) \Theta\left(t+t_{d}-n t_{p}\right) \\
& \times\left\langle 0\left|\left(\prod_{m=0}^{N} \hat{a}_{1}^{(m)} \hat{b}_{2}^{(m)}\right) \hat{a}_{3}^{(k) \dagger} \hat{b}_{4}^{(n) \dagger} \hat{b}_{4}^{(n)} \hat{a}_{3}^{(k)}\left(\prod_{m=0}^{N} \hat{a}_{1}^{(m) \dagger} \hat{b}_{2}^{(m) \dagger}\right)\right| 0\right\rangle
\end{aligned}
$$


Writing the operators for modes 3 and 4 in terms of 1 and 2 yields:

$$
\begin{aligned}
& 2_{1 a, 2 b}\left\langle\psi_{N}\left|E_{3 a}^{-}(t) E_{4 b}^{-}\left(t+t_{d}\right) E_{4 b}^{+}\left(t+t_{d}\right) E_{3 a}^{+}(t)\right| \psi_{N}\right\rangle_{1 a, 2 b} \\
= & \frac{1}{2 \tau^{2}} \sum_{k=0}^{N} \sum_{n=0}^{N} e^{-\left(t-k t_{p}\right) / \tau} e^{-\left(t+t_{d}-n t_{p}\right) / \tau} \Theta\left(t-k t_{p}\right) \Theta\left(t+t_{d}-n t_{p}\right) \\
& \times\langle 0|\left(\prod_{m=0}^{N} \hat{a}_{1}^{(m)} \hat{b}_{2}^{(m)}\right)\left(\hat{a}_{1}^{(k) \dagger}-\hat{a}_{2}^{(k) \dagger}\right)\left(\hat{b}_{1}^{(n) \dagger}+\hat{b}_{2}^{(n) \dagger}\right) \\
& \times\left(\hat{b}_{1}^{(n)}+\hat{b}_{2}^{(n)}\right)\left(\hat{a}_{1}^{(k)}-\hat{a}_{2}^{(k)}\right)\left(\prod_{m=0}^{N} \hat{a}_{1}^{(m) \dagger} \hat{b}_{2}^{(m) \dagger}\right)|0\rangle \\
= & \frac{1}{2 \tau^{2}} \sum_{k=0}^{N} \sum_{n=0}^{N} e^{-\left(t-k t_{p}\right) / \tau} e^{-\left(t+t_{d}-n t_{p}\right) / \tau} \Theta\left(t-k t_{p}\right) \Theta\left(t+t_{d}-n t_{p}\right) \\
& \times\left\langle 0\left|\left(\prod_{m=0}^{N} \hat{a}_{1}^{(m)} \hat{b}_{2}^{(m)}\right) \hat{a}_{1}^{(k) \dagger} \hat{b}_{2}^{(n) \dagger} \hat{b}_{2}^{(n)} \hat{a}_{1}^{(k)}\left(\prod_{m=0}^{N} \hat{a}_{1}^{(m) \dagger} \hat{b}_{2}^{(m) \dagger}\right)\right| 0\right\rangle \\
= & \frac{1}{2 \tau^{2}} \sum_{k=0}^{N} \sum_{n=0}^{N} e^{-\left(t-k t_{p}\right) / \tau} e^{-\left(t+t_{d}-n t_{p}\right) / \tau} \Theta\left(t-k t_{p}\right) \Theta\left(t+t_{d}-n t_{p}\right)
\end{aligned}
$$

Plugging this into Eq. 5.20 and integrating over $t$ (again, integrals identical to

Eq. 5.10), we obtain the final form for the second-order correlation function for distinguishable photons.

$$
P_{2 p h, J t o t}\left(t_{d}\right)=\frac{1}{4 \tau} \sum_{k=0}^{N} \sum_{n=0}^{N}\left(2-\delta_{k n}\right) e^{-\left|t_{d}+t_{p}(k-n)\right| / \tau}
$$

This result for distinguishable photons impinging on the beamsplitter is plotted against the case of identical photons (Eq. 5.18) in Fig. 5.4.

With a clear understanding of the expected signal, we can now delve into the experimental results.

\subsection{Experimental interference of photons at a beamsplitter}

The interference of single photons at a beamsplitter was accomplished by using two trapped ${ }^{174} \mathrm{Yb}^{+}$atoms as single photon emitters. A single ytterbium ion is confined in each of two nearly identical four-rod rf traps that are contained in independent 


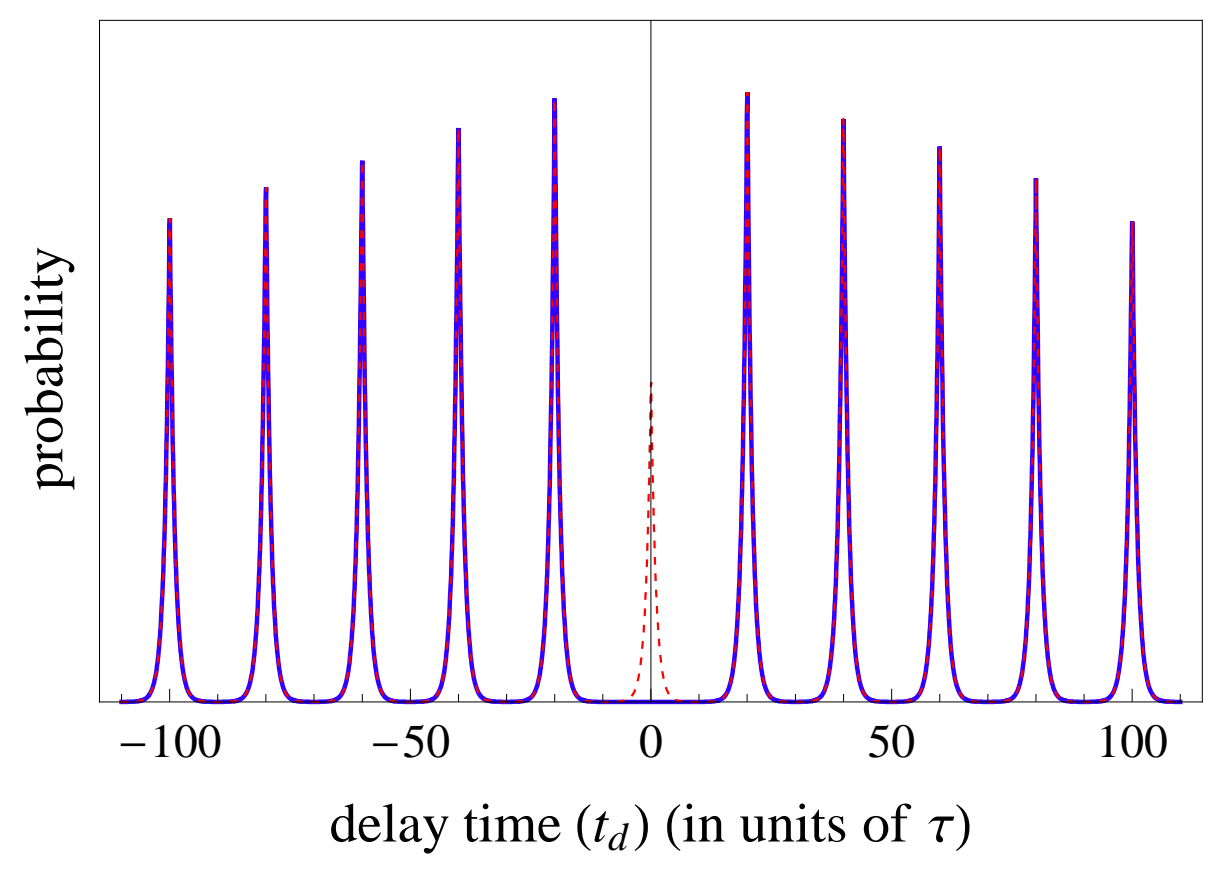

Figure 5.4: Theoretical two-photon second-order correlation function, for identical (blue, solid line; Eq. 5.18) and distinguishable (red, dotted line; Eq. 5.23) photons. Plotted is the relative probability of detecting a photon in both mode 3 and mode 4 as a function of the time delay $\left(t_{d}\right)$ between the two photons for $N=20$. The repetition rate shown is about 20 times the natural lifetime of the atomic excited state $(\tau)$. Interference between identical photons impinging on the beamsplitter result in no detections at zero time delay $t_{d}=0$ (blue, solid line). In contrast, distinguishable photons do not interfere, and thus coincident detections are allowed, as evidenced by the peak at $t_{d}=0$ (red, dotted line). 
vacuum chambers. As described in the previous chapter, the ions are Dopplercooled by cw laser light at $369.53 \mathrm{~nm}$, and the presence of a single atom in each trap is confirmed by imaging this fluorescence on a camera. In addition to the objective lens used to observe the ion fluorescence on the camera, we position a second objective of numerical aperture 0.23 on the other side of the vacuum chamber, as illustrated in Fig. 5.5. Photons collected by this objective are coupled into a singlemode optical fiber and directed to a 50:50 non-polarizing beamsplitter. ${ }^{4}$ The output of the fiber is incident on the beamsplitter at a small angle (about $10^{\circ}$ ) to ensure the optic is polarization independent. The photons are detected by one of two PMTs, positioned at each exit port of the beamsplitter. Polarizers on electronic flip-mounts can be added to the beam path between the beamsplitter and each PMT to enable detection of only identically (parallel) polarized photons. Conversely, distinguisable (perpendicularly polarized) photons are detected by the addition of a $\lambda / 2$-waveplate to one of these beam paths. The arrival times of the photons are recorded by a time-to-digital converter (TDC) with 4 ps resolution. ${ }^{5}$

Ultrafast excitation of an $\mathrm{Yb}^{+}$atom from ${ }^{2} S_{1 / 2}$ to ${ }^{2} P_{1 / 2}$ should result in the generation of a single spontaneously emitted photon. We use pulses of about 1 ps duration with central wavelength at $369.5 \mathrm{~nm}$ to drive the ${ }^{2} S_{1 / 2} \leftrightarrow{ }^{2} P_{1 / 2}$ transition in the atom. Since the duration of the pulse is much shorter than the excited state lifetime (8.12 ns), the probability that the atom scatters two photons during a single pulse is approximately given by the probability of one spontaneous decay over the duration of the pulse: $1-e^{-(1 \mathrm{ps}) /(8.12 \mathrm{~ns})} \approx 10^{-4}$.

The 1 ps ultrafast pulses are produced by an actively mode-locked Ti:sapphire laser (Spectra-Physics Tsunami) operating at a central wavelength of $739 \mathrm{~nm}$ and a

\footnotetext{
${ }^{4}$ The optical fiber is StockerYale NUV-320-K1. The fiber is single-mode at $370 \mathrm{~nm}$, with a specified attenuation of approximately $0.1 \mathrm{~dB} /$ meter.

${ }^{5}$ The TDC is a PicoHarp, made by PicoQuant.
} 


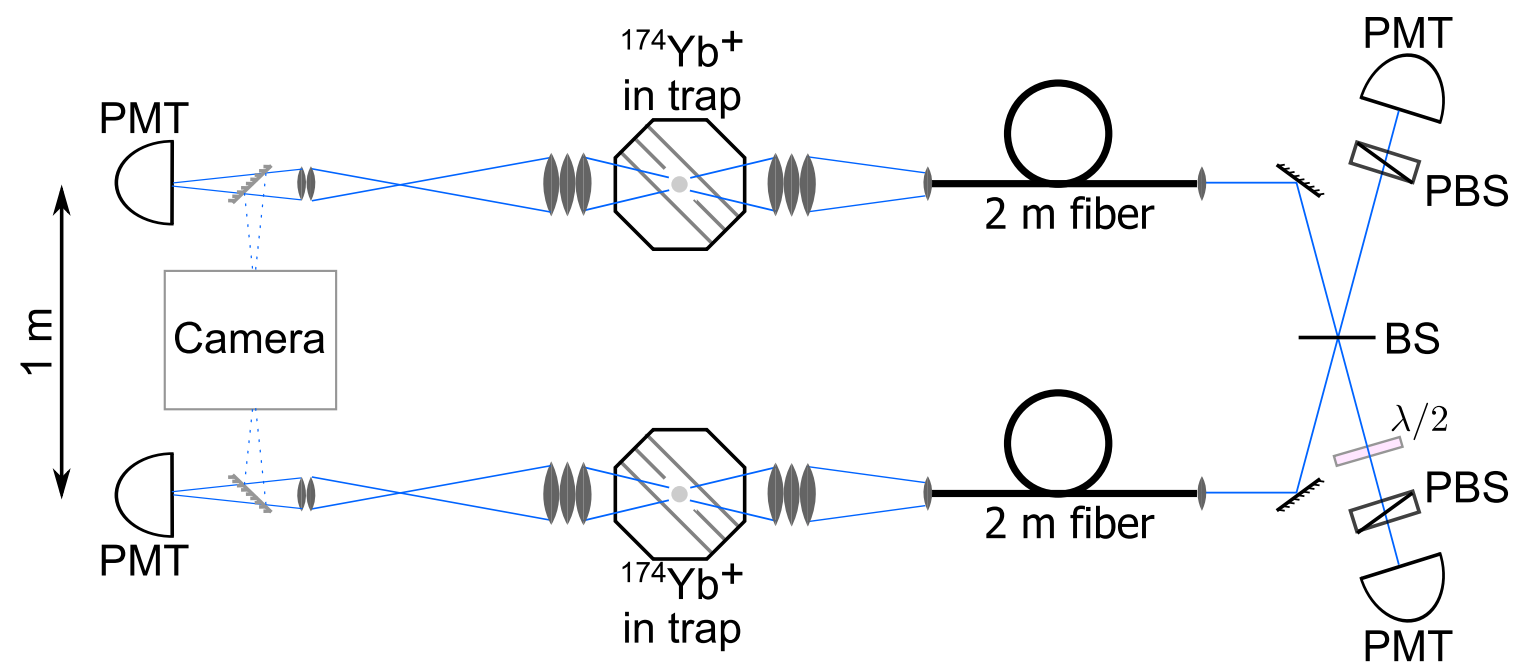

Figure 5.5: Experimental setup for the two-photon interference experiment. Fluorescence of an $\mathrm{Yb}^{+}$atom during Doppler cooling can be monitored by either a photon-counting photomultiplier (PMT) or a camera (the grayed mirror is removable by an electronic flipmount). The second-order correlation function (joint detection probability) is measured by coupling spontaneously emitted photons from the ion into a single-mode optical fiber, the output of which is directed to a 50:50 nonpolarizing beamsplitter (BS). Polarizers (PBS) and a $\lambda / 2$-waveplate $(\lambda / 2)$ are removable for the measurement of unpolarized, parallel polarized, or perpendicularly polarized photons.

repetition rate of about $81 \mathrm{MHz}$. This pulse laser is pumped by approximately 5.75 $\mathrm{W}$ of $532 \mathrm{~nm}$ light (Spectra-Physics Millennia), producing an average output power of $1 \mathrm{~W}$ at $739 \mathrm{~nm}$. We measured the width of the pulses to be 1 ps by using a simple autocorrelator, consisting of a Michelson interferometer and a lithium triborate (LBO) crystal with a photodetector. The pulses are passed through an electro-optic pulse picker that has an average extinction ratio better than 100:1 in the infrared. With the pulse picker synced to the intracavity acousto-optic modulator (AOM) of the pulse laser, we reduce the effective pulse repetition rate to about $8.1 \mathrm{MHz}$ by allowing only one in every ten pulses to pass. A critically phase-matched LBO crystal is used to frequency double each pulse, resulting in approximately $80 \mathrm{~mW}$ average power at $369.5 \mathrm{~nm}$. This second-harmonic light is separated from the fundamental infrared by a prism. In addition to creating the wavelength needed to resonantly ex- 


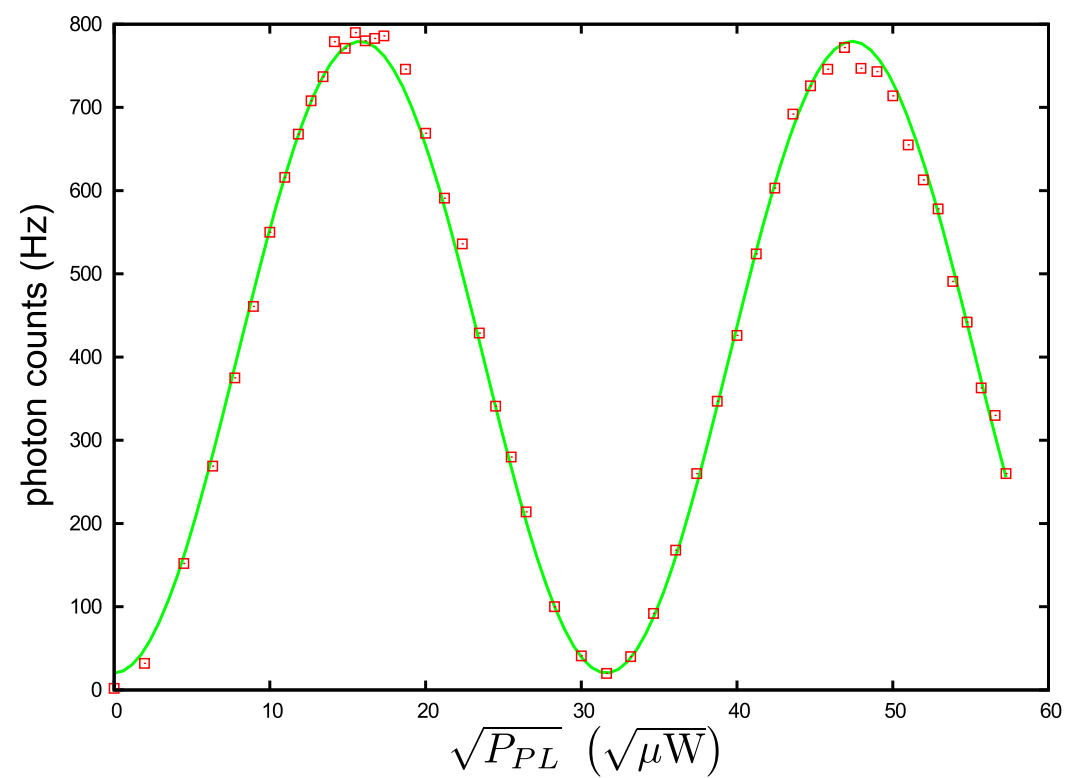

Figure 5.6: Picosecond Rabi flopping. The scattering rate of a single ${ }^{174} \mathrm{Yb}^{+}$atom is monitored as a train of 1 ps pulses of variable power excite the atom. The Rabi frequency for driving the atom from ${ }^{2} S_{1 / 2}$ to ${ }^{2} P_{1 / 2}$ is proportional to the square-root of the incident pulse power. At maximum pulse energy, we achieve about a $3.5 \pi$ rotation. The green curve is a fit to the function $A \sin ^{2}\left(B \sqrt{P_{P L}} / 2\right)$, where $P_{P L}$ is the average power of the pulsed laser incident on the ion, and $A$ and $B$ are the fit parameters.

cite the ion, frequency doubling increases the effective average extinction ratio of the pulse picker to about $10^{4}: 1{ }^{6}$ Even though the second-harmonic generation efficiency is only about $8 \%$, the resulting pulses at $369.5 \mathrm{~nm}$ have more than enough power to excite an atom with unit probability. This is demonstrated in Fig. 5.6, where the scattering rate of a single atom is measured as a function of the incident pulse power, indicating each pulse has enough power to drive about a $3.5 \pi$ rotation between the ${ }^{2} S_{1 / 2}$ and ${ }^{2} P_{1 / 2}$ levels. As the Rabi frequency is proportional to the square root of the incident pulse power, this means that a single pulse could be split into about 12 pulses, each with enough power to drive a $\pi$ rotation [76]. In the experiments presented here and in the following chapters, each pulse is attenuated to achieve approximately unit excitation probability ( $1 \pi$ pulse).

\footnotetext{
${ }^{6}$ The immediately trailing pulse is not extinquished as well as the other pulses (only about $10^{3}: 1$ ), due to the finite switching of the bias voltage applied to the pulse picker. Averaging over all pulses though yields an extinction ratio of approximately $10^{4}: 1$.
} 
Before looking at the interference of two photons, we first demonstrate that the ultrafast excitation of a single $\mathrm{Yb}^{+}$atom is an excellent source of single photons. For this measurement, only the photons scattered by one of the two ions is incident on the beamspitter (the other is blocked). The experiment consists of a repetitive sequence of $10 \mu \mathrm{s}$ of cooling followed by $40 \mu \mathrm{s}$ of excitation/measurement. During the cooling interval, the pulse picker is switched off so that the ion is illuminated solely by the cw lasers used for Doppler cooling. For the excitation/measurement interval the $369.53 \mathrm{~nm} \mathrm{cw}$ light is switched off by an AOM, and the pulse picker is gated open to allow a train of 1 ps pulses, separated in time by about 124 ns, to sequentially excite the atom from ${ }^{2} S_{1 / 2}$ to ${ }^{2} P_{1 / 2}$. After each excitation, the atom will spontaneously decay while emitting a single photon. Emitted photons at 369.53 $\mathrm{nm}$ are collected by the objective lens, coupled into the single-mode optical fiber, directed onto the beamsplitter, and detected by the PMTs. The arrivals times of the detected photons allow us to determine the joint detection probability, shown in Fig. 5.7. The functional form of the experimental joint detection probability matches the theorectical single-photon source illustrated previously (Fig. 5.3), with the lack of a peak at delay time $t_{d}=0$ indicating that at most a single photon is emitted after each ultrafast excitation pulse [69]. Dark counts on the PMTs result in a small background contribution at all delay times; coupling the light from the atom into a single-mode fiber highly suppresses the contribution of background scattered light.

We observe the quantum two-photon interference effect by simultaneously exciting both trapped $\mathrm{Yb}^{+}$atoms, and combining the two emitted photons on the beamsplitter. $^{7}$ The joint probability of detection for identical photons is measured by

\footnotetext{
${ }^{7}$ Of course, simultaneously excitation is not essential; we just need the emitted photons to arrive at the beamsplitter simultaneously. As such, the path length of the excitation pulses are adjusted so that emitted photons from each ion arrive at the beamsplitter within 100 ps of each other. Since the path lengths between each ion and the beamsplitter are approximately equal, this means the ions are also excited at about the same time.
} 


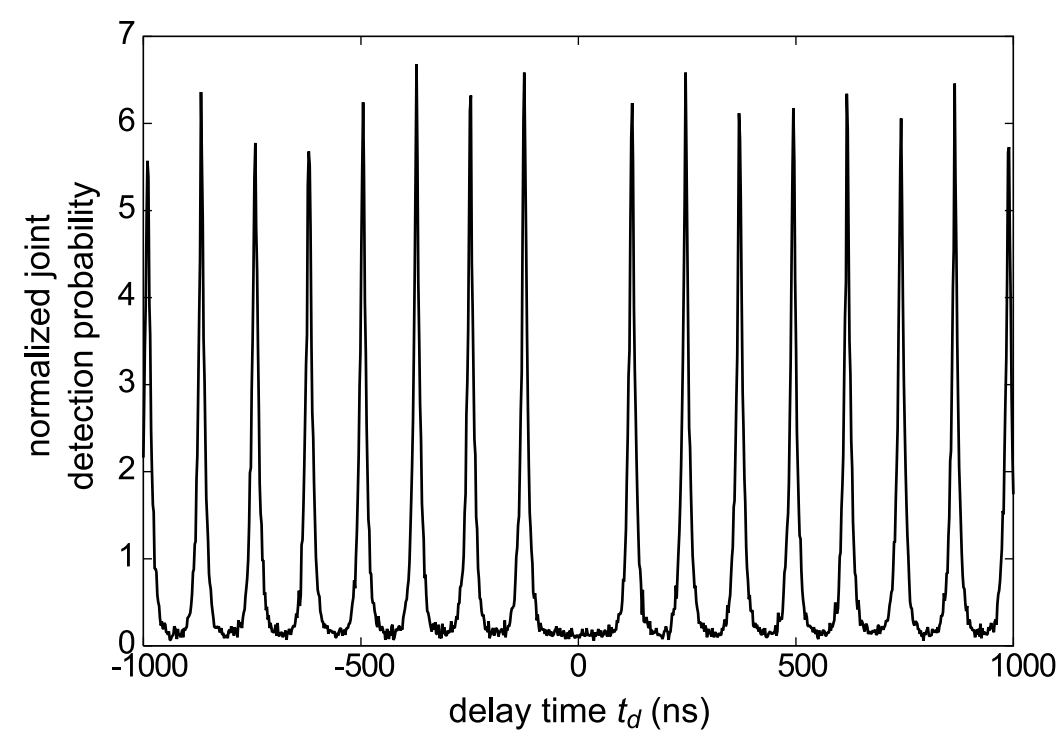

Figure 5.7: Normalized experimental single-photon second-order correlation function. The lack of a peak at time delay $t_{d}=0$ is indicative of a source of single photons, confirming that at most one photon is emitted by the atom following ultrafast excitation [69].

placing both polarizers in the beam path, while detection of distinguishable photons is evaluated by including the $\lambda / 2$-waveplate in one of the two paths. As was seen theoretically in Fig. 5.4, for identical photons we expect no detections at time delay $t_{d}=0$, while for distinguishable photons we expect a probability of joint detection half as large as that from adjacent excitations. The data shown in Fig. 5.8 demonstrates this quantum two-photon interference effect. In the measurement of parallel polarized photons, the residual counts at time delay $t_{d}=0$ result from both dark counts on the PMTs, and imperfect spatial mode overlap of the photons on the beamsplitter. The data shown in Fig. 5.8 corresponds to an interferometer contrast of approximately 95\% [69]. The spatial filtering afforded by coupling the spontaneously emitted photons into single-mode fibers was essential to achieving this excellent two-photon interference.

The single-photon source and quantum interference effect demonstrated here are an essential components of the probabilistic, heralded quantum gate that is used to 


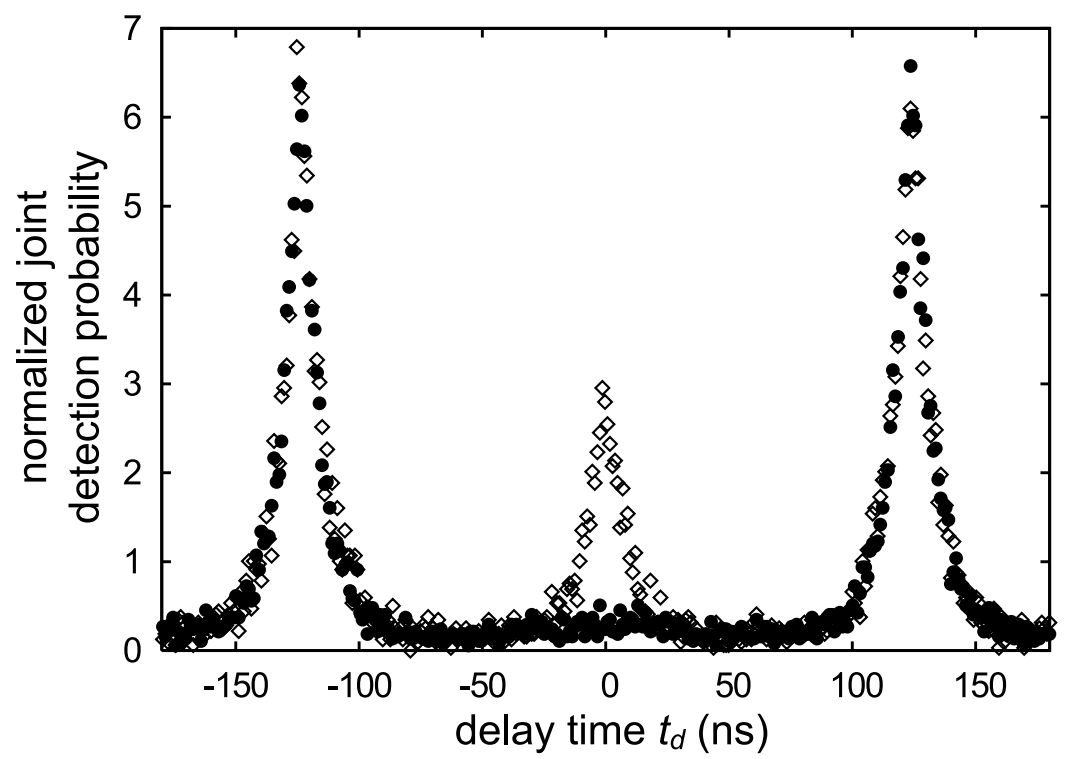

Figure 5.8: Normalized experimental two-photon second-order correlation function for identical (parallel polarized) photons (diamonds) and distinguishable (perpendicularly polarized) photons (circles) [69]. As expected from the theorectical calculations of Sec. 5.1, two identical photons incident on the beamsplitter always exit by the same port, resulting suppression of joint detections at time delay $t_{d}=0$.

implement the teleportation protocol. 


\section{CHAPTER VI}

\section{Lifetime of the ${ }^{2} P_{1 / 2}$ level in $\mathbf{Y b}^{+}$}

\footnotetext{
"I want to share something with you: the three little sentences that will get you through life. Number 1: Cover for me. Number 2: Oh, good idea, Boss! Number 3: It was like that when I got here."

-Homer Simpson, The Simpsons
}

Measurements of the properties of individual atoms are constantly being pursued. Precise atomic measurements are used for a multitude of applications, including tests of fundamental physical theories. The ytterbium ion, in particular, is a remarkable, versatile atomic system. In addition to its use in quantum information and computation, $\mathrm{Yb}^{+}$has been noted for the implementation of atomic frequency standards $[35,81,116,117,118]$ and tests of ab initio atomic structure calculations [119]. The spectrum of $\mathrm{Yb}^{+}$is also important in astrophysics for the of study main sequence stars [120] and the solar photosphere [121, 122].

In this chapter, we present a precise measurement of the lifetime of the $6 p^{2} P_{1 / 2}$ excited state of $\mathrm{Yb}^{+}$. While not strictly necessary for the teleportation protocol, the experiment is naturally related to the two-photon interference experiment of the previous chapter, as the charateristic shape of the peaks in the second-order correlation function are determined by the natural lifetime of the excited state. In addition, measuring this excited state lifetime yields information concerning the temporal aspects of the spontaneously emitted photons. 
Previous methods used to measure the excited states of $\mathrm{Yb}^{+}$include delayed coincidence [123, 124], beam-foil [125], Hanle effect [125, 126], laser-induced fluorescence of sputtered metal vapor [127] and laser-induced plasma [128], beam-laser [87, 129], and quantum jumps in single ions $[86,85]$. Here, we measure the $6 p^{2} P_{1 / 2}$ excited state of the $\mathrm{Yb}^{+}$atom using a time-correlated single photon-counting technique [130] adapted to utilize the features of our single atom system [131]. By using ultrafast pulses to excite a single trapped $\mathrm{Yb}^{+}$atom and coupling the emitted photons into a fiber, we were able to eliminate many of the systematics present in earlier measurements. The lifetime of the $6 p^{2} P_{1 / 2}$ state was measured to be $8.12 \pm 0.02 \mathrm{~ns}$.

\subsection{Lifetime measurement}

A single ${ }^{174} \mathrm{Yb}^{+}$atom is trapped and Doppler cooled, as described previously IV. A diagram of the experimental setup for the lifetime measurement is shown in Fig. 6.1. As in the previous chapter, the 1 ps pulses produced by the actively mode-locked Ti:sapphire laser are passed through an electro-optic pulse picker to control the pulse repetition rate. In this experiment, the repetition rate is reduced to about 5.5 $\mathrm{MHz}$ by allowing only one in every fifteen pulses to pass. Essential to the lifetime measurement is the extinction ratio of the pulse picker; as before, the effective average extinction ratio of the pulse picker is about $10^{4}: 1$.

In this experiment, the ion is first Doppler cooled for $200 \mu \mathrm{s}$, and then the cw $369.5 \mathrm{~nm}$ light is blocked. The electro-optic pulse picker is then gated open for 390 $\mu \mathrm{s}$, allowing a train of pulses to pass, where each subsequent pulse is separated from the preceding by about 180 ns. Leakage of each pulse (in the infrared) through a mirror strikes a trigger diode, which sends an electric pulse to one channel of the TDC with a resolution of 4 ps. Each frequency-doubled laser pulse excites the ion 


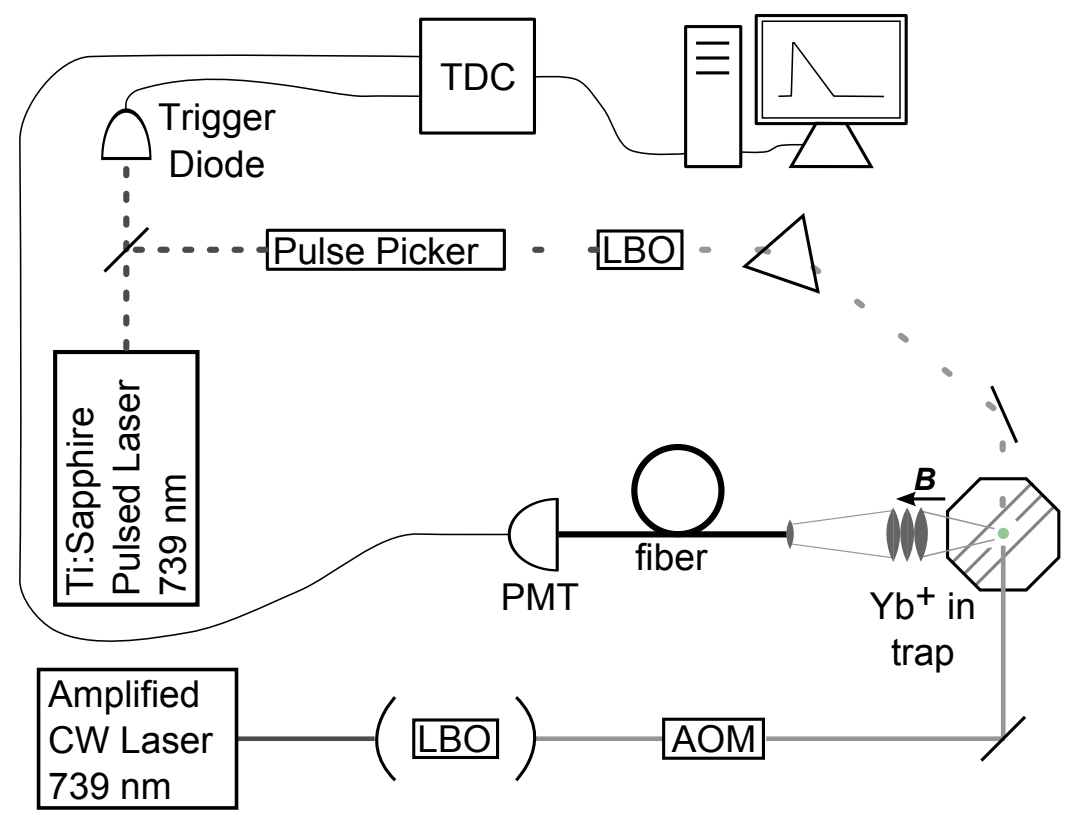

Figure 6.1: The experimental setup used to determine the lifetime of the $\mathrm{Yb}^{+} 6 p^{2} P_{1 / 2}$ excited state. The $935.2 \mathrm{~nm}$ and $638.6 \mathrm{~nm}$ lasers used for depopulating the metastable ${ }^{2} D_{3 / 2}$ and ${ }^{2} F_{7 / 2}$ states, respectively, are not shown. LBO is a lithium triborate nonlinear crystal; AOM is an acousto-optic modulator; TDC is a time-to-digital converter; PMT is a photomultiplier tube; and $\boldsymbol{B}$ is the applied magnetic field.

from ${ }^{2} S_{1 / 2}$ to ${ }^{2} P_{1 / 2}$ with near unit probability. As seen in Chap. V the subsequent spontaneous decay of the excited state produces a single photon [69]. The photons emitted by the ion are collected with an objective lens with numerical aperature 0.23 , coupled into a single-mode fiber, and detected by a PMT. The arrival time of the consequent electric pulses from the PMT are recorded by the second channel of the TDC.

A histogram of the time difference between the arrivals of the two electric pulses at the TDC displays the charateristic exponential decay of the excited atomic state (Fig. 6.2(a)). However, several other factors contribute to the overall shape of the histogram. The pulse propagation time (of both the light and electric pulses) results in an overall offset along the time axis. Background counts result from PMT triggers due to either a background scattered photon or a dark count. In fact, it might be expected that background photons are most likely to be detected as a result of the 
laser pulse traversing the trap, producing a "prompt peak" in the data at the time of excitation. Moreover, the finite response time of the PMT dictates that the observed data is a convolution of all of these effects with the instrument response function. Thus, the data shown in Fig. 6.2(a) is described by the function:

$$
\begin{aligned}
F(t) & =\sum_{t_{n}}\left[A e^{-\left(t_{n}-t_{0}\right) / \tau} \Theta\left(t_{n}-t_{0}\right)+B+C \delta_{n 0}\right] h\left(t-t_{n}\right) \\
& =B+C h\left(t-t_{0}\right)+\sum_{t_{n}} A e^{-\left(t_{n}-t_{0}\right) / \tau} \Theta\left(t_{n}-t_{0}\right) h\left(t-t_{n}\right)
\end{aligned}
$$

where $A$ is the amplitude of the exponential decay of the atomic state, $B$ is the background counts, $C$ is the amplitude of the "prompt peak," $h$ is the normalized instrument response function, and $t_{0}$ is the time of excitation (used as a fit parameter, but shown in Fig. 6.2 as $\left.t_{0}=0\right)$. The sum is over all possible time bins $t_{n}$ used in the histogram of the data.

We measure the instrument response function by coupling a small fraction of the light from the $369.5 \mathrm{~nm}$ pulse into an optical fiber, and directing the output onto the PMT. Since the duration of the pulse (1 ps) is much smaller than the response time of the PMT (order nanosecond), the pulse is effectively a delta function input, allowing us to directly measure the instrument response function. The measured instrument response function, $h$, is shown in Fig. 6.2(b). Also visible on the log-scale plot are the subsequent, highly suppressed pulses from the mode-locked laser (average extinction ratio of about $10^{4}: 1$ ), separated by the approximately $12 \mathrm{~ns}$ repetition rate. ${ }^{1}$

The differential nonlinearity of the TDC is characterized by directing light from a flashlight to the fiber-coupler, and integrating this "white noise" input for several hours. The differential nonlinearity of the TDC is found to result in intermittent ripples with amplitude $<6 \%$ peak-to-peak. We use this measured signal (after

\footnotetext{
${ }^{1}$ While the suppressed, trailing pulses are not strictly part of the instrument response function, the effect of these pulses is essentially the same as if they had been generated electronically, so we will continue to call this measurement the instrument response function of the system, and treat it as such.
} 


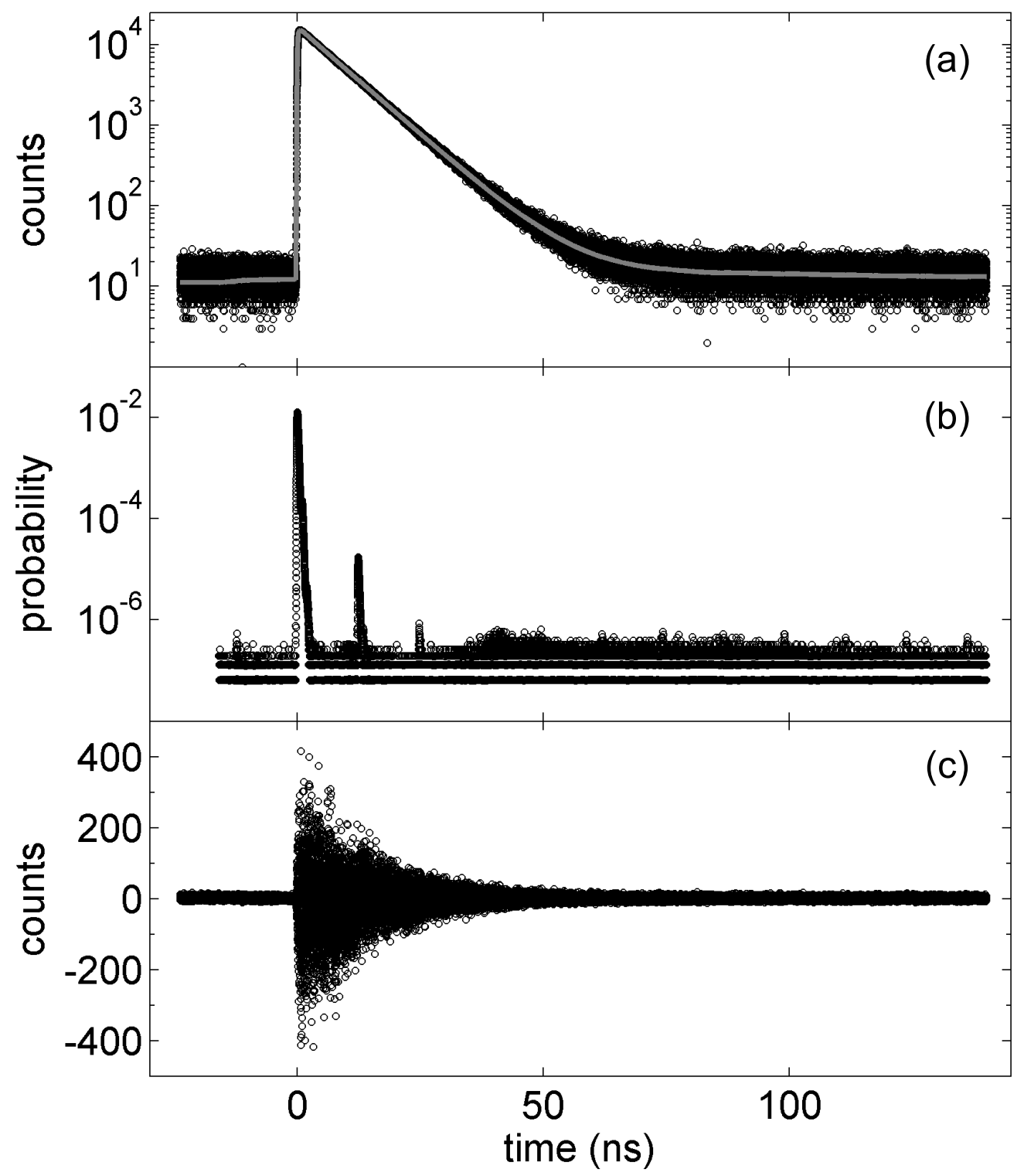

Figure 6.2: Data used to determine the lifetime of the $6{ }^{2} P_{1 / 2}$ excited state. (a) The data after correction for the differential nonlinearity of the TDC, with 4 ps binning, showing the number of photons (stop pulses) detected a given time following pulsed laser excitation (start pulses); the gray line is the fit, with functional form given by Eq. 6.1. (b) The normalized instrument response function. Visible on the log-scale plot are the highly suppressed pulses from the mode-locked laser (average extinction ratio of about $10^{4}: 1$ ), separated by the repetition rate of about 12 ns. (c) Deviations of the data from the fitting function (residuals). 
normalization) to correct for the differential nonlinearity of the TDC by dividing the data (lifetime and instrument response measurements) by this signal.

The data analysis consists of computing Eq. 6.1 for a range of values for $A$, $B, C$, and $t_{0}$, and comparing this function to the data to determine $\chi^{2}$ for each combination of parameters. The final fit is shown as the gray line in Fig. 6.2(a), and the deviations of the data from this fit (residuals) are presented in Fig. 6.2(c). The statistical uncertainty in the fit is $0.002 \mathrm{~ns}$. The quoted uncertainty in the lifetime is dominated by systematic errors.

Our experimental setup allows us to eliminate many possible systematics. By using a single trapped atom, systematic errors such as pulse pileup, radiation trapping, subradiance and superradiance are eliminated $[132,133]$. Exciting this atom with an ultrafast pulse eliminates effects due to the application of light during the measurement interval, including background scattered light, multiple excitations, and ac Stark shifts. Using the pulse picker to reduce the effective repetition rate of the ultrafast laser enabled observation intervals much longer than the natural decay time of the excited state, allowing a fit of the data all the way to the tale end of the exponential where residual background events dominate. Finally, by coupling the spontaneously emitted photons into a single mode fiber, we nearly eliminate detection of background scattered light, including "prompt peak" photons scattered while the ultrafast pulse traverses the trapping region.

Two possible systematics that demand further investigation, though, are hyperfine and Zeeman quantum beats. 


\subsection{Quantum beats}

Quantum beats arise from a coherent superposition of excited states decaying to a common final state. The quantum decay channels can then interfere and produce a modulation in the fluorescence signal at the frequency difference of the two decay channels. Quantum beats could thereby alter the observed fluorescence decay, shifting the measured value of the excited state lifetime.

The fluorescence signal from the atom, including the contribution of quantum beats, is given by $[134]$

$$
I(t) \propto\left(\alpha^{2} a^{2}+\beta^{2} b^{2}+2 \alpha \beta a b \cos \left(\omega_{q b} t\right)\right) e^{-t / \tau}
$$

and thus the modulation depth of the quantum beat signal is

$$
\eta_{q b}=\frac{2 \alpha \beta a b}{\left(\alpha^{2} a^{2}+\beta^{2} b^{2}\right)}
$$

where $\alpha$ and $\beta$ are the matrix elements of the two excitation paths for a given polarization of the excitation light; $a$ and $b$ are the matrix elements for decay to a common final state while producing a photon of a particular polarization; $\omega_{q b}$ is the frequency difference of the two excited states; and $\tau$ is the natural lifetime of the excited states (here assumed to be the same for both excited states). The effect of quantum beats on the fluoresence decay signal from the atom for a variety of parameters is shown in Fig. 6.3.

By using an even isotope of ytterbium (nuclear spin 0), we avoid the possibility of hyperfine beats. On the other hand, at a magnetic field of 3.4 gauss the excited state Zeeman splitting is $3.1 \mathrm{MHz}$, so Zeeman beats could be significant. In order to

minimize the effect of quantum beats in the measurement of the lifetime of the ${ }^{2} P_{1 / 2}$ level in $\mathrm{Yb}^{+}$, we carefully adjusted two experimental parameters: (1) we attempted 

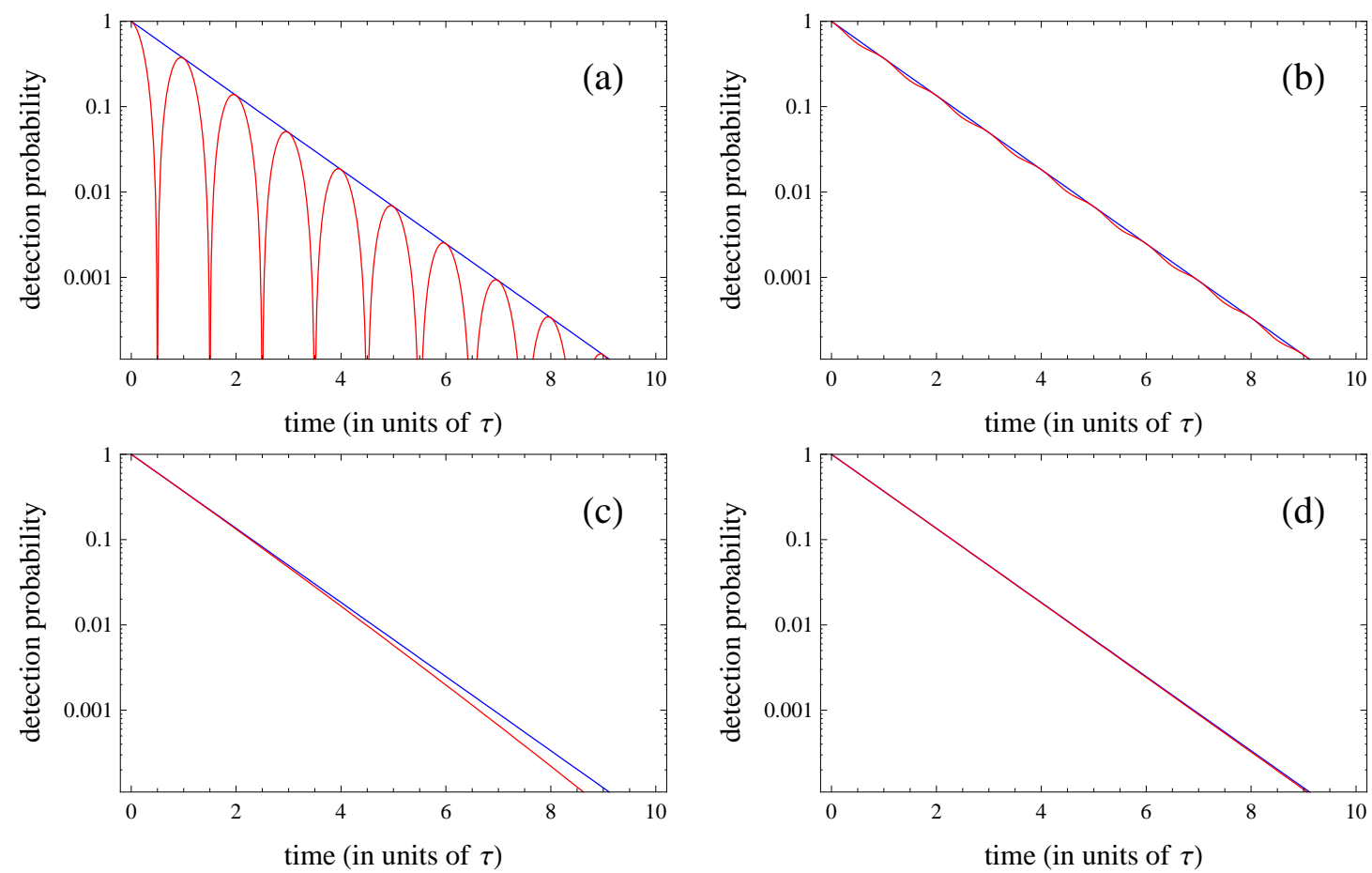

Figure 6.3: Theoretical quantum beats, given by Eq. 6.2. The figure shows the relative probability of detecting a photon as a function of time (in units of the lifetime $\tau$ of the excited state) with (red) and without (blue) quantum beats: (a) with full amplitude $(\beta=\alpha=a=$ $b=1 / \sqrt{2})$ and period equal to the natural lifetime $(\omega=2 \pi / \tau)$; (b) with experimentally measured amplitude upperbound $(\beta=0.026)$ and period equal to the natural lifetime; (c) with full amplitude and experimentally determined period $(\omega=2 \pi /(40.2 \tau))$; and (d) with experimentally measured amplitude $(\beta=0.026)$ and period $(\omega=2 \pi /(40.2 \tau))$. 
to excite the ion using purely $\pi$-polarized light to prevent producing coherences in the excited ${ }^{2} P_{1 / 2}$ manifold; and (2) we collected photons emitted along the quantization axis, so that the collected photons were (ideally) $\sigma$-polarized only, and thereby only observe decay channels which result in separate final states. In the ideal case, either of these considerations would eliminate a quantum beat signal. Of course, in the actual experiment these measures are not perfect, so we need to characterize the expected quantum beat signal.

Although the polarization of the pulsed laser is carefully aligned with respect to the magnetic field to induce only $\pi$-transitions in the atom, we estimate that the polarization of the laser could be misaligned by as much as 1 degree. Given the strength of the $\sigma$-transition is twice that of the $\pi$-transition, this then leads to as much as $2\left|\sin \left(\pi \frac{1}{45}\right)\right|^{2}=0.01$ of the total population being excited to the incorrect Zeeman level in ${ }^{2} P_{1 / 2}$. This residual coherence could then result in the formation of a quantum beat.

Due to the known numerical aperture of the observation optics $(\mathrm{NA}=0.23)$, we know that about $1.3 \%$ of all collected photons result from a $\pi$-decay (App. D). Although coupling the collected photons into a single-mode fiber should eliminate the contribution of $\pi$-polarized photons, due to possible misalignment of the quantization axis with the observation axis, some contribution is expected.

An upperbound on the modulation depth of the quantum beat signal can be obtained experimentally by measuring a novel single-photon second-order correlation function. The setup for this correlation measurement is similar to that shown in Chap. V, with two PMTs measuring the exit ports of a 50:50 beamsplitter, but with laser polarization and magnetic field alignment as presented here. For this measurement, a quarter-wave plate is inserted between the ion and the fiber-coupler, and 
polarizers are placed before the PMTs for detection of only $\sigma^{+}$-polarized light after the beamsplitter (the $\lambda / 2$-waveplate shown in Fig. 5.5 is removed). Given detection of a $\sigma^{+}$-polarized photon, since the excitation light is $\pi$-polarized, the immediately previous/subsequent spontaneously emitted photon should not be detected; this results in suppression of the first adjacent peaks of the correlation measurement (at delay times of $\pm 180 \mathrm{~ns}$ ), as seen in Fig. 6.4. The same errors that contribute to the generation of a quantum beat signal cause the first adjacent peaks of this second-order correlation function to be nonzero. However, while in the second-order correlation signal the probabilities for excitation to the wrong Zeeman level and detection of $\pi$-polarized photons add, in the quantum beat signal the amplitudes of these effects are multiplied [134]. The quantum beat signal would therefore be maximal if these errors contribute equally to the height of the first adjacent peaks in the second-order correlation signal, allowing us to place an upperbound on the modulation depth of the quantum beat signal of $<0.03$. Given this modulation depth, and a period of oscillation determined by the Zeeman splitting, we determine quantum beats shift the measured lifetime of the excited state by less than $\pm 0.002 \mathrm{~ns}$.

\subsection{Conclusion}

Ultimately, the dominant systematic error was determined to be the residual ripple in the data due to the differential nonlinearity of the TDC. We estimate the contribution to the error by truncating the data at various intervals in the decay/ripple and performing a fit (Eq. 6.1) on this truncated data set. The fitted value of lifetime shifts as the truncation point is varied. We therefore assign a systematic error large enough to encompass all of these shifted values (although this is almost certainly an overestimate of the error). The estimated systematic error due to the residual 


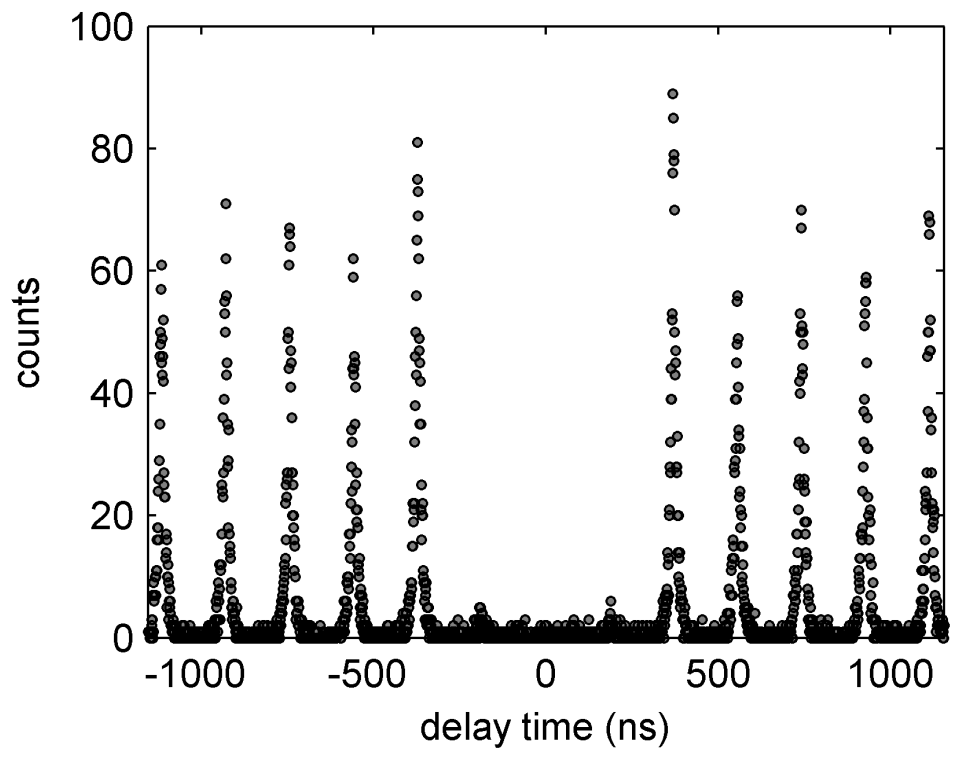

Figure 6.4: Second-order correlation function used to determine the amplitude of quantum beats. Through a combination of a quarter-wave plate and polarizers, we arrange the setup to observe $\sigma^{+}$-polarized light after a beamsplitter. In using $\pi$-polarized pulses for excitation, then two subsequent excitations should not both result in detection of a $\sigma^{+}$-polarized photon. This is evidenced by the suppression of the first adjacent peaks in the joint detection probability (at delay times of $\pm 180 \mathrm{~ns}$ ).

differential nonlinearity of the TDC is then $\pm 0.015 \mathrm{~ns}$.

The final value of the lifetime of the $6 p^{2} P_{1 / 2}$ state of $\mathrm{Yb}^{+}$is measured to be $8.12 \pm 0.02$ ns. In Fig. 6.5, our measurement is plotted alongside other experimental and theoretical values. As is apparent in the figure, our result is consistent with prior measurements, with an improvement in the uncertainty by almost a factor of five. 


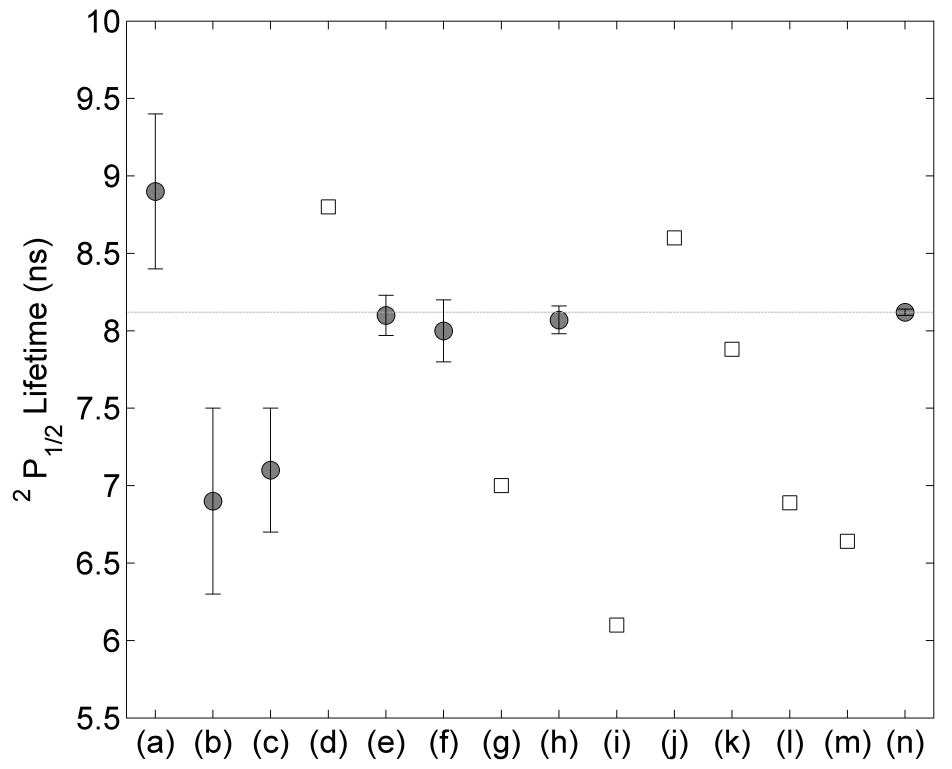

Figure 6.5: Comparison of values obtained for $6 p^{2} P_{1 / 2}$ state lifetime. (a) M. L. Burshtein et al. (1974), expt: delayed-coincidence [124]; (b) T. Andersen et al. (1975), expt: beamfoil [125]; (c) K. B. Blagoev et al. (1978), expt: delayed-coincidence [123]; (d) B. C. Fawcett et al. (1991), theory: Hartree-Fock [94]; (e) R. W. Berends et al. (1993), expt: beam-laser [87]; (f) R. M. Lowe et al. (1993), expt: laser-induced fluorescence [127]; (g) R. M. Lowe et al. (1993), theory: many-body perturbation theory [127]; (h) E. H. Pinnington et al. (1997), expt: beam-laser [129]; (i) E. H. Pinnington et al. (1997), theory: Coulomb approximation calculation [129]; (j) E. Biémont et al. (1998), theory: Hartree-Fock [89]; (k) E. Biémont et al. (2002), theory: Hartree-Fock [135]; (l) U. I. Safronova et al. (2009), theory: relativistic many-body perturbation theory [136]; (m) U. I. Safronova et al. (2009), theory: relativistic many-body perturbation theory [136]; (n) this work (also gray dashed line). 


\section{CHAPTER VII}

\section{Quantum Gate}

Arthur looked up. "Ford!" he said, "there's an infinite number of monkeys outside who want to talk to us about this script for Hamlet they've worked out."

-Douglas Adams, The Hitchhiker's Guide to the Galaxy

One of the vital components to quantum computation left to demonstrate in this work is a scalable two-qubit quantum gate. The standard model of quantum computation is the quantum circuit model, where a series of gate operations are performed and at the output the qubits are measured as a final step. The quantum circuit model is being pursued by several research groups using trapped atomic ions. The deterministic, two-qubit gates that utilize the common modes of motion of the trapped ions have already achieved an impressive $99.3 \%$ fidelity [56]. Effort in this area is focused on scaling the system to larger numbers of qubits, as can be done, for instance, by constructing complex trap arrays where ions can be stored and interacted as various intervals and locations [18]. Crucial to the success of this methodology will be an understanding of the motional decoherence that plagues all ion traps $[41,23,24,25]$.

Another approach to scalable quantum computation is the cluster-state (or oneway) quantum computing model, where the measurement process itself is an integral part of gate operation [137]. In this scheme, a large entangled state is first prepared. The computation is then executed by successive measurements of the qubits, with 
classical feed-forward of the measurements determining the single-qubit gates to be performed on subsequent sets of qubits before measurement. This is an entirely new approach that has been proven to be equivalent to the circuit model, and allows for a new class of quantum gates to be considered for scalable quantum computation: non-unitary, measurement-based gates.

Basic quantum operations applicable to the cluster-state model of quantum computation have been carried out using entangled photons [138, 139]. However, the photonic cluster states in these experiments were generated by spontaneous parametric down-conversion, relied on post-selection, and did not utilize a quantum memory, making these implementations difficult to scale to larger systems [140].

We present the implementation of a probabilistic, heralded quantum gate between two remote quantum memories. The gate is based on the interference and detection of two photons [66]. Even though the gate is probabilistic, the incorporation of a quantum memory allows this system to be efficiently scaled, potentially allowing for the generation of the large cluster-states necessary for one-way quantum computation $[141,66,142]$. Moreover, since the operation is mediated by photons, this gate used in any systems of optically active qubits, such as neutral atoms, ions, nitrogenvacancy centers, or quantum dots. Indeed, hybrid systems are also envisioned, where disparate quantum systems are connected via this photon-mediated process to exploit the advantages of each individual quantum system.

In this implementation of the heralded quantum gate, two $\mathrm{Yb}^{+}$atoms at a distance of about one meter are used as quantum memories [143]. The photon-mediated approach to scalable quantum computing with trapped atomic ions has the advantage that complex trap arrays may not be required, and that the operation is insensitive to motional decoherence. In addition, the gate can be operated on qubits separated 


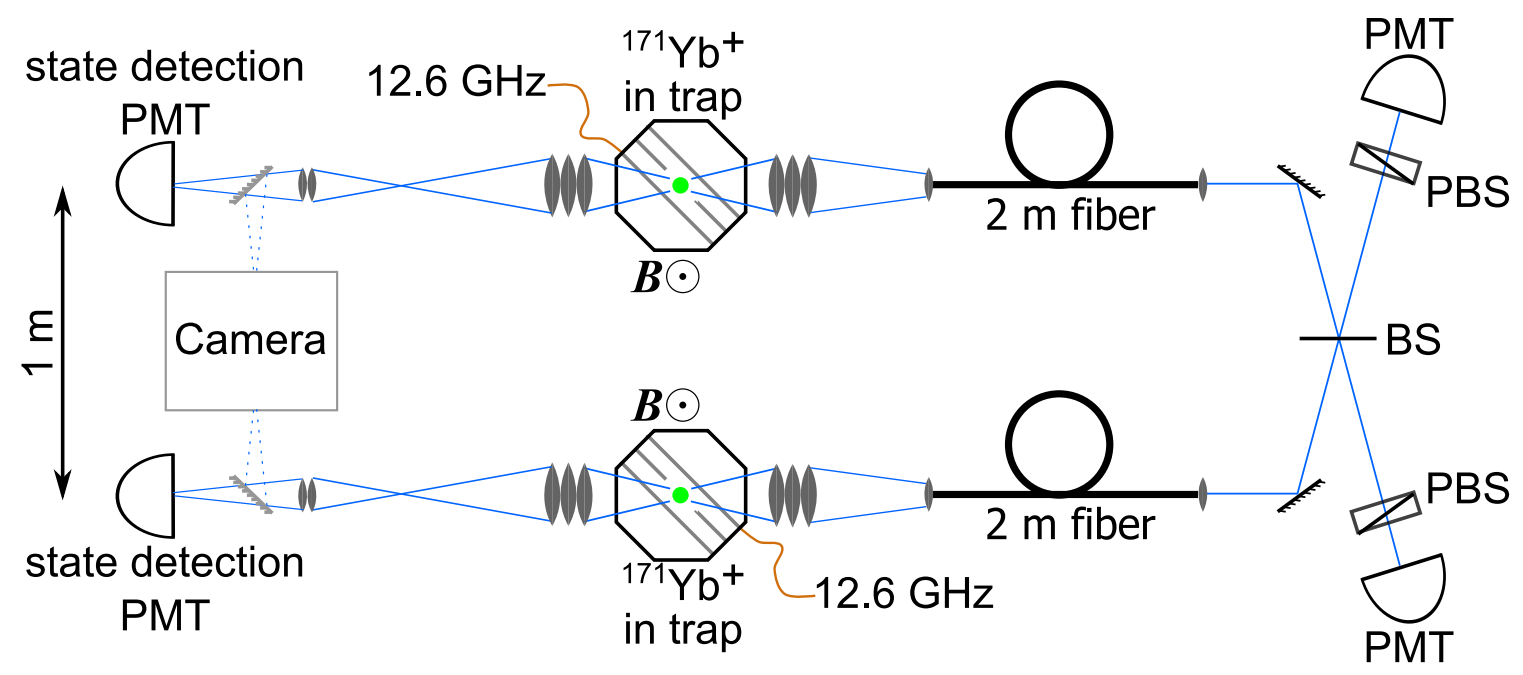

Figure 7.1: Experimental setup for the heralded quantum gate. Spontaneously emitted $\pi$-polarized photons are coupled in a single-mode fiber and directed to interfere on a 50:50 nonpolarizing beamsplitter (BS). Coincident detection of two photons by photon-counting photomultipliers (PMTs) announce the success of the gate between the two ions. PBS are polarizers used to filter the photons so that only $\pi$-polarized photons are detected. The state of each ion is measured by state-dependent fluorescence, detected by a PMT on the opposite side of the vacuum chamber.

by an arbitrary distance.

\subsection{Ion-photon entanglement}

The implementation of the gate begins by confining and cooling two ${ }^{171} \mathrm{Yb}^{+}$atoms in two vacuum chambers, separated by a distance of about one meter. The experimental setup, shown in Fig. 7.1, is similar to that employed for the two-photon interference experiments of the previous chapter. In this case, though, the ions are subjected to an external magnetic field of about 5.2 gauss that is aligned perpendicular to both the observation axis and the impinging light from the picosecond pulsed laser. Also, the polarizers are present in the interferometer setup, whereas the optional $\lambda / 2$-waveplate used previously is removed from the beam path.

After Doppler cooling, each ion is initialized to the state $|0\rangle$ by optical pumping. Each of the two ions, ion $a$ and ion $b$, are then prepared in a particular quantum 
state by resonant microwave radiation at $12.6 \mathrm{GHz}$ applied directly to one of the trap electrodes. As was derived in Chap. IV, this allows us to prepare the ions in any state. Thus, after application of the microwave radiation we write the state of the atoms as

$$
\begin{aligned}
|\psi\rangle_{a} & =\alpha|0\rangle_{a}+\beta|1\rangle_{a} \\
|\psi\rangle_{b} & =\gamma|0\rangle_{b}+\delta|1\rangle_{b}
\end{aligned}
$$

where the only restriction on $\alpha, \beta, \gamma$, and $\delta$ are that $|\alpha|^{2}+|\beta|^{2}=1$ and $|\gamma|^{2}+|\delta|^{2}=1$. For clarity, we will assume ideal state evolution throughout this discussion; for the full phase evolution during the gate, see App. E.

After this state preparation, each ion is excited with near-unit probability to the ${ }^{2} P_{1 / 2}$ level by an ultrafast (1 ps) laser pulse having linear polarization aligned parallel to the magnetic field ( $\pi$-polarized) and central wavelength at $369.5 \mathrm{~nm}$. Due to the polarization of the pulse and atomic selection rules, the broadband pulse coherently drives $|0\rangle$ to $\left|0^{\prime}\right\rangle:={ }^{2} P_{1 / 2}\left|F=1, m_{F}=0\right\rangle$ and $|1\rangle$ to $\left|1^{\prime}\right\rangle:={ }^{2} P_{1 / 2}\left|F=0, m_{F}=0\right\rangle$, as illustrated in Fig. 7.2(a). Since the bandwidth of the 1 ps pulse is approximately $300 \mathrm{GHz}$, we are able to drive both of these transitions simultaneously [76]. The $100 \mathrm{THz}$ fine structure splitting of the ${ }^{2} P$ levels ensures that coupling to the ${ }^{2} P_{3 / 2}$ is negligible.

As seen in Chap. V, each ion spontaneously emits only a single photon at 369.5 nm while returning to the ${ }^{2} S_{1 / 2}$ ground state. The emitted photons at $369.5 \mathrm{~nm}$ can each be collected along a direction perpendicular to the quantization axis by objective lenses of numerical aperture $\mathrm{NA}=0.23$ and coupled into single-mode fibers. Observation along this direction allows for polarization filtering of the emitted photons because those produced by $\pi$ and $\sigma$ transitions appear as orthogonally polarized [13]. 


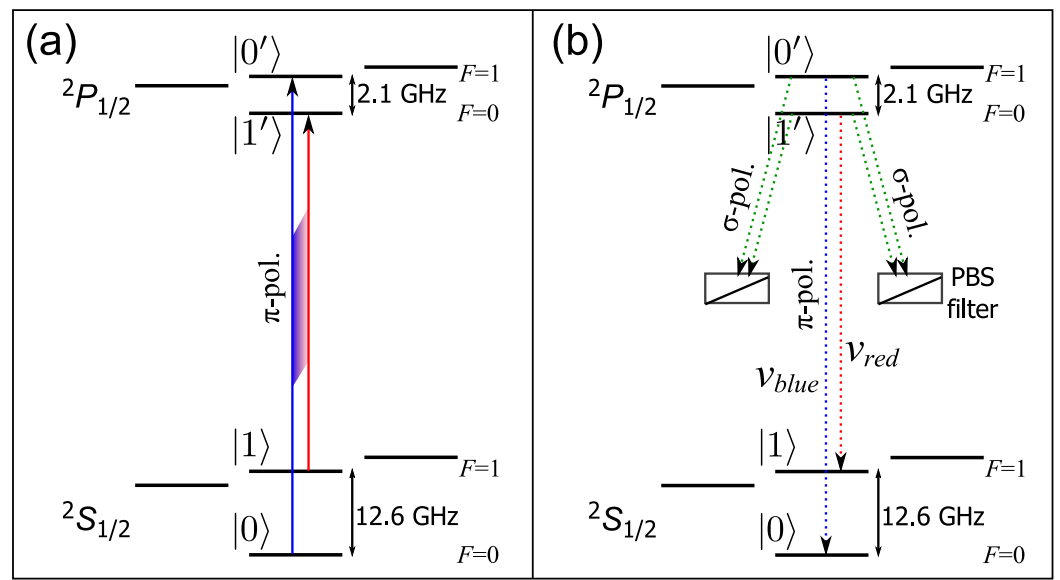

Figure 7.2: Pulsed excitation with $\pi$-polarized light to generate ion-photon entanglement. (a) Due to the atomic selection rules, $\pi$-polarized light drives $|0\rangle$ to $\left|0^{\prime}\right\rangle$ and $|1\rangle$ to $\left|1^{\prime}\right\rangle$. The pulse bandwidth of about $300 \mathrm{GHz}$ allows both of these transitions to be driven simultaneously with near-unit excitation probability for each. (b) After excitation, the ion spontaneously decays back to ${ }^{2} S_{1 / 2}$ and emits a single photon at $369.5 \mathrm{~nm}$. If we consider only $\pi$-polarized photons, then the frequency of the emitted photon is entangled with the internal electronic state of the atom, with the separation of the different frequency modes equal to the sum of the ${ }^{2} S_{1 / 2}$ and ${ }^{2} P_{1 / 2}$ hyperfine splittings $\left(\nu_{\text {blue }}-\nu_{\text {red }}=14.7 \mathrm{GHz}\right)$. Polarizers (PBS) are used to filter out the $\sigma$-polarized light.

Considering only $\pi$ decays results in each ion being entangled with the frequency of its emitted photon such that

$$
\begin{aligned}
|\psi\rangle_{a} & =\alpha|0\rangle_{a}\left|\nu_{\text {blue }}\right\rangle_{a}+\beta|1\rangle_{a}\left|\nu_{\text {red }}\right\rangle_{a} \\
|\psi\rangle_{b} & =\gamma|0\rangle_{b}\left|\nu_{b l u e}\right\rangle_{b}+\delta|1\rangle_{b}\left|\nu_{\text {red }}\right\rangle_{b}
\end{aligned}
$$

where $\left|\nu_{\text {blue }}\right\rangle$ and $\left|\nu_{\text {red }}\right\rangle$ are the single photon states having well-resolved frequencies $\nu_{\text {blue }}$ and $\nu_{\text {red }}$, respectively. The bandwidth of each state is determined by the natural linewidth of the excited state to be $1 /(2 \pi \tau)=19.6 \mathrm{MHz}$ (Chap. VI). The two frequency states are separated by the sum of the ${ }^{2} S_{1 / 2}$ and ${ }^{2} P_{1 / 2}$ hyperfine splittings, so that $\nu_{b l u e}-\nu_{\text {red }}=14.7 \mathrm{GHz}$. The output of the single-mode fibers is then directed to interfere at a 50:50 nonpolarizing beamsplitter. 


\subsection{Ion-ion entanglement}

Although we looked extensively at the action of the beamsplitter on identical and distinguishable photons in Chap. V, the case of superposition states yields a somewhat different result. In order to evaluate the action of the beamsplitter in the present scenario, we first rewrite the state of the two ion-photon systems in the Bell state basis introduced in Chap. II. The total state of the two ion-photon systems is simply the product of the states in Eq. 7.2.

$$
\begin{aligned}
|\Psi\rangle= & |\psi\rangle_{a} \otimes|\psi\rangle_{b} \\
= & \left(\alpha|0\rangle_{a}\left|\nu_{b l u e}\right\rangle_{a}+\beta|1\rangle_{a}\left|\nu_{\text {red }}\right\rangle_{a}\right) \otimes\left(\gamma|0\rangle_{b}\left|\nu_{b l u e}\right\rangle_{b}+\delta|1\rangle_{b}\left|\nu_{\text {red }}\right\rangle_{b}\right) \\
= & \alpha \gamma|0\rangle_{a}|0\rangle_{b}\left|\nu_{b l u e}\right\rangle_{a}\left|\nu_{b l u e}\right\rangle_{b}+\alpha \delta|0\rangle_{a}|1\rangle_{b}\left|\nu_{b l u e}\right\rangle_{a}\left|\nu_{\text {red }}\right\rangle_{b} \\
& +\beta \gamma|1\rangle_{a}|0\rangle_{b}\left|\nu_{\text {red }}\right\rangle_{a}\left|\nu_{b l u e}\right\rangle_{b}+\beta \delta|1\rangle_{a}|1\rangle_{b}\left|\nu_{\text {red }}\right\rangle_{a}\left|\nu_{\text {red }}\right\rangle_{b} \\
= & \alpha \gamma|0\rangle_{a}|0\rangle_{b} \frac{1}{\sqrt{2}}\left(\left|\phi^{+}\right\rangle_{p h}+\left|\phi^{-}\right\rangle_{p h}\right)+\alpha \delta|0\rangle_{a}|1\rangle_{b} \frac{1}{\sqrt{2}}\left(\left|\psi^{+}\right\rangle_{p h}+\left|\psi^{-}\right\rangle_{p h}\right) \\
& +\beta \gamma|1\rangle_{a}|0\rangle_{b} \frac{1}{\sqrt{2}}\left(\left|\psi^{+}\right\rangle_{p h}-\left|\psi^{-}\right\rangle_{p h}\right)+\beta \delta|1\rangle_{a}|1\rangle_{b} \frac{1}{\sqrt{2}}\left(\left|\phi^{+}\right\rangle_{p h}-\left|\phi^{-}\right\rangle_{p h}\right) \\
= & \frac{1}{\sqrt{2}}\left[\left|\phi^{+}\right\rangle_{p h}\left(\alpha \gamma|0\rangle_{a}|0\rangle_{b}+\beta \delta|1\rangle_{a}|1\rangle_{b}\right)+\left|\phi^{-}\right\rangle_{p h}\left(\alpha \gamma|0\rangle_{a}|0\rangle_{b}-\beta \delta|1\rangle_{a}|1\rangle_{b}\right)\right. \\
& \left.+\left|\psi^{+}\right\rangle_{p h}\left(\alpha \delta|0\rangle_{a}|1\rangle_{b}+\beta \gamma|1\rangle_{a}|0\rangle_{b}\right)+\left|\psi^{-}\right\rangle_{p h}\left(\alpha \delta|0\rangle_{a}|1\rangle_{b}-\beta \gamma|1\rangle_{a}|0\rangle_{b}\right)\right](7.3
\end{aligned}
$$

Thus, we just need to determine the action of the beamsplitter on the four possible Bell states. The evaluation will use the same terminology of Chap. V, in the simple ideal case. ${ }^{1}$ Assuming photon $a$ enters the beamsplitter via port 1 and photon $b$ enters the beamsplitter by port 2 , and associating the creation operators $\hat{a}^{\dagger}$ and $\hat{b}^{\dagger}$

\footnotetext{
${ }^{1}$ The spatial effect of the electric field operators was considered in calculating the error do to mode mismatch at the beamsplitter.
} 
with frequency modes $\left|\nu_{\text {red }}\right\rangle$ and $\left|\nu_{\text {blue }}\right\rangle$, respectively, we then find:

$$
\begin{aligned}
\left|\phi^{ \pm}\right\rangle_{p h} & =\frac{1}{\sqrt{2}}\left[\left|\nu_{\text {blue }}\right\rangle_{a}\left|\nu_{\text {blue }}\right\rangle_{b} \pm\left|\nu_{\text {red }}\right\rangle_{a}\left|\nu_{\text {red }}\right\rangle_{b}\right] \\
& =\frac{1}{\sqrt{2}}\left[\hat{b}_{1}^{\dagger} \hat{b}_{2}^{\dagger} \pm \hat{a}_{1}^{\dagger} \hat{a}_{2}^{\dagger}\right]|0\rangle_{p h} \\
& =\frac{1}{2 \sqrt{2}}\left[\left(\hat{b}_{3}^{\dagger}+\hat{b}_{4}^{\dagger}\right)\left(-\hat{b}_{3}^{\dagger}+\hat{b}_{4}^{\dagger}\right) \pm\left(\hat{a}_{3}^{\dagger}+\hat{a}_{4}^{\dagger}\right)\left(-\hat{a}_{3}^{\dagger}+\hat{a}_{4}^{\dagger}\right)\right]|0\rangle_{p h} \\
& =\frac{1}{2 \sqrt{2}}\left[-\left(\hat{b}_{3}^{\dagger}\right)^{2}-\hat{b}_{4}^{\dagger} \hat{b}_{3}^{\dagger}+\hat{b}_{3}^{\dagger} \hat{b}_{4}^{\dagger}+\left(\hat{b}_{4}^{\dagger}\right)^{2} \mp\left(\hat{a}_{3}^{\dagger}\right)^{2} \mp \hat{a}_{4}^{\dagger} \hat{a}_{3}^{\dagger} \pm \hat{a}_{3}^{\dagger} \hat{a}_{4}^{\dagger} \pm\left(\hat{a}_{4}^{\dagger}\right)^{2}\right]|0\rangle_{p h} \\
& =\frac{1}{2}\left[-\left|2\left(\nu_{\text {blue }}\right)_{3} 0_{4}\right\rangle+\left|0_{3} 2\left(\nu_{\text {blue }}\right)_{4}\right\rangle \mp\left|2\left(\nu_{\text {red }}\right)_{3} 0_{4}\right\rangle \pm\left|0_{3} 2\left(\nu_{\text {red }}\right)_{4}\right\rangle\right]
\end{aligned}
$$

for the $\left|\phi^{ \pm}\right\rangle_{p h}$ state, where in the last line the notation shows the number of photons of a particular frequency in the spatial mode 3 or 4 . Similarly, for the $\left|\psi^{+}\right\rangle_{p h}$ and $\left|\psi^{-}\right\rangle_{p h}$ photon states, we find:

$$
\begin{aligned}
\left|\psi^{+}\right\rangle_{p h} & =\frac{1}{\sqrt{2}}\left[\left|\nu_{\text {blue }}\right\rangle_{a}\left|\nu_{\text {red }}\right\rangle_{b}+\left|\nu_{\text {red }}\right\rangle_{a}\left|\nu_{\text {blue }}\right\rangle_{b}\right] \\
& =\frac{1}{\sqrt{2}}\left[\hat{b}_{1}^{\dagger} \hat{a}_{2}^{\dagger}+\hat{a}_{1}^{\dagger} \hat{b}_{2}^{\dagger}\right]|0\rangle_{p h} \\
& =\frac{1}{2 \sqrt{2}}\left[\left(\hat{b}_{3}^{\dagger}+\hat{b}_{4}^{\dagger}\right)\left(-\hat{a}_{3}^{\dagger}+\hat{a}_{4}^{\dagger}\right)+\left(\hat{a}_{3}^{\dagger}+\hat{a}_{4}^{\dagger}\right)\left(-\hat{b}_{3}^{\dagger}+\hat{b}_{4}^{\dagger}\right)\right]|0\rangle_{p h} \\
& =\frac{1}{2 \sqrt{2}}\left[-\hat{b}_{3}^{\dagger} \hat{a}_{3}^{\dagger}-\hat{b}_{4}^{\dagger} \hat{a}_{3}^{\dagger}+\hat{b}_{3}^{\dagger} \hat{a}_{4}^{\dagger}+\hat{b}_{4}^{\dagger} \hat{a}_{4}^{\dagger}-\hat{b}_{3}^{\dagger} \hat{a}_{3}^{\dagger}-\hat{b}_{3}^{\dagger} \hat{a}_{4}^{\dagger}+\hat{b}_{4}^{\dagger} \hat{a}_{3}^{\dagger}+\hat{b}_{4}^{\dagger} \hat{a}_{4}^{\dagger}\right]|0\rangle_{p h} \\
& =\frac{1}{\sqrt{2}}\left[\left|0_{3}\left(\nu_{b l u e} \nu_{\text {red }}\right)_{4}\right\rangle-\left|\left(\nu_{\text {blue }} \nu_{\text {red }}\right)_{3} 0_{4}\right\rangle\right]
\end{aligned}
$$

and

$$
\begin{aligned}
\left|\psi^{-}\right\rangle_{p h} & =\frac{1}{\sqrt{2}}\left[\left|\nu_{\text {blue }}\right\rangle_{a}\left|\nu_{\text {red }}\right\rangle_{b}-\left|\nu_{\text {red }}\right\rangle_{a}\left|\nu_{\text {blue }}\right\rangle_{b}\right] \\
& =\frac{1}{\sqrt{2}}\left[\hat{b}_{1}^{\dagger} \hat{a}_{2}^{\dagger}-\hat{a}_{1}^{\dagger} \hat{b}_{2}^{\dagger}\right]|0\rangle_{p h} \\
& =\frac{1}{2 \sqrt{2}}\left[\left(\hat{b}_{3}^{\dagger}+\hat{b}_{4}^{\dagger}\right)\left(-\hat{a}_{3}^{\dagger}+\hat{a}_{4}^{\dagger}\right)-\left(\hat{a}_{3}^{\dagger}+\hat{a}_{4}^{\dagger}\right)\left(-\hat{b}_{3}^{\dagger}+\hat{b}_{4}^{\dagger}\right)\right]|0\rangle_{p h} \\
& =\frac{1}{2 \sqrt{2}}\left[-\hat{b}_{3}^{\dagger} \hat{a}_{3}^{\dagger}-\hat{b}_{4}^{\dagger} \hat{a}_{3}^{\dagger}+\hat{b}_{3}^{\dagger} \hat{a}_{4}^{\dagger}+\hat{b}_{4}^{\dagger} \hat{a}_{4}^{\dagger}+\hat{b}_{3}^{\dagger} \hat{a}_{3}^{\dagger}+\hat{b}_{3}^{\dagger} \hat{a}_{4}^{\dagger}-\hat{b}_{4}^{\dagger} \hat{a}_{3}^{\dagger}-\hat{b}_{4}^{\dagger} \hat{a}_{4}^{\dagger}\right]|0\rangle_{p h} \\
& =\frac{1}{\sqrt{2}}\left[\left|\left(\nu_{\text {blue }}\right)_{3}\left(\nu_{\text {red }}\right)_{4}\right\rangle-\left|\left(\nu_{\text {red }}\right)_{3}\left(\nu_{\text {blue }}\right)_{4}\right\rangle\right]
\end{aligned}
$$

The calculations above show the striking result that the only one of the four Bell states that results in a single photon in both exit ports of the beamsplitter is the 
antisymmetric, $\left|\psi^{-}\right\rangle_{p h}$ state [144]. This crucial point is the final step in the quantum gate.

We use the joint detection of two photons at the exit ports of the beamsplitter to herald the success of the quantum gate. By the above derivation, a coincident detection occurs signals that the photon were in the $\left|\psi^{-}\right\rangle_{p h}$ state. From Eq. 7.3, we see that the coincident detection thereby projects the ions into the state:

$$
|\psi\rangle_{i o n s}={ }_{p h}\left\langle\psi^{-} \mid \Psi\right\rangle=\frac{1}{\sqrt{2 \vartheta}}\left(\alpha \delta|0\rangle_{a}|1\rangle_{b}-\beta \gamma|1\rangle_{a}|0\rangle_{b}\right)
$$

where the factor $\vartheta=\left(|\alpha|^{2}|\delta|^{2}+|\beta|^{2}|\gamma|^{2}\right) / 2$ in front is the usual renormalization term present after a measurement [31]. In the case $|\alpha|=|\beta|=|\gamma|=|\delta|=1 / \sqrt{2}$, the ions are left in a maximally entangled state. In terms of operators, this gate is written as

$$
\frac{1}{2} \sigma_{3}^{a}\left(\sigma_{0}^{a} \sigma_{0}^{b}-\sigma_{3}^{a} \sigma_{3}^{b}\right)
$$

where $\sigma_{j}^{i}$ is the $j$ th Pauli operator acting on the $i$ th qubit [66].

In contrast to our earlier demonstrations of remote ion entanglement [70, 145], the initial state amplitudes are preserved by the heralded quantum gate and determine the form of the final ion-ion entangled state. This is a defining feature of a gate operation, and is essential to establishing entanglement between more than two qubits. However, unlike the CNOT gate introduced in Chap. II, the quantum gate presented here is not unitary. Indeed, for certain initial states (e.g. $\alpha=\gamma=1$ ), a coincident detection should never occur. While this behavior voids its application to the quantum circuit model, cluster states can still be generated by having all qubits initially in a superposition state. In this case, the gate succeeds with nonvanishing probability, and scales favorably [66]. 


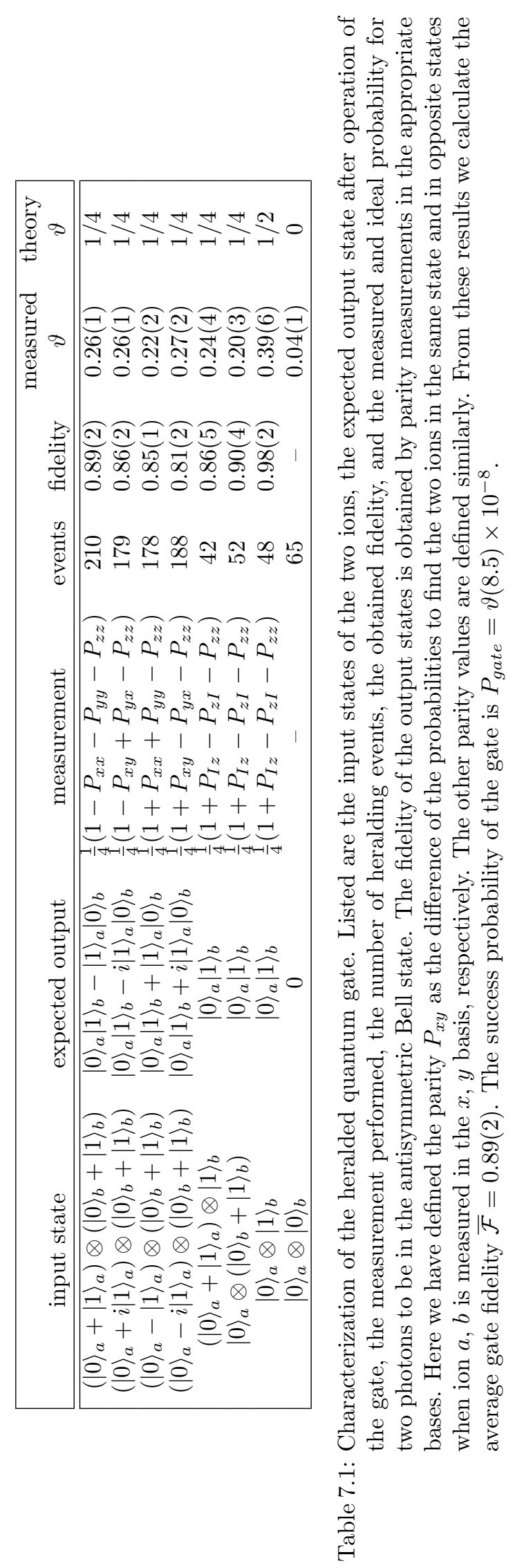




\subsection{Gate evaluation}

We verify the operation of the gate by implementing it on a variety of input states. The gate is characterized by determining the fidelity of the resulting output state with respect to the ideal case described by Eq. 7.7. The fidelity is defined as the overlap of the measured state with the ideal state:

$$
\mathcal{F}=\left.\left.\right|_{\text {ideal }}\langle\psi \mid \psi\rangle\right|^{2}={ }_{\text {ideal }}\langle\psi|\rho| \psi\rangle_{\text {ideal }}
$$

where we have written our measured state as $|\psi\rangle$ and defined the density matrix $\rho=|\psi\rangle\langle\psi|$. The various input states used to characterize the quantum gate, together with the measurements made and the resulting fidelities for each, are given in Table 7.1. As measurement of each ion is accomplished using the aforementioned state fluorescence technique (Chap. IV), measurement in the remaining two bases requires an additional microwave pulse before detection. As such, we define the rotation $\left\{R_{y}(\pi / 2), R_{x}(\pi / 2), R(0)\right\}$ before detection to correspond to measurement in the basis $\{x, y, z\}$. Overall, we attain an average fidelity of 89(2)\% [143].

To evaluate the gate, we do not make measurements of all combinations of possible input states around the equator of the Bloch sphere. The reason for this is that input states that differ only by a global phase rotation are indistinguishable in the lab. In other words, it is only the phase difference between the states that matters experimentally, where the microwave oscillator is used as a reference. As an example, consider the two possible combinations of input states $1 / \sqrt{2}\left(|0\rangle_{a}+|1\rangle_{a}\right)$ and $1 / \sqrt{2}\left(|0\rangle_{b}+i|1\rangle_{b}\right)$ versus $1 / \sqrt{2}\left(|0\rangle_{a}+i|1\rangle_{a}\right)$ and $1 / \sqrt{2}\left(-|0\rangle_{b}+|1\rangle_{b}\right)$. Experimentally, the way both of these states are implemented is to apply a microwave $\pi / 2$-pulse to ion $a$, and apply a microwave $\pi / 2$-pulse to ion $b$ that is $+\pi / 2$ out of phase with the pulse that was applied to ion $a$. 

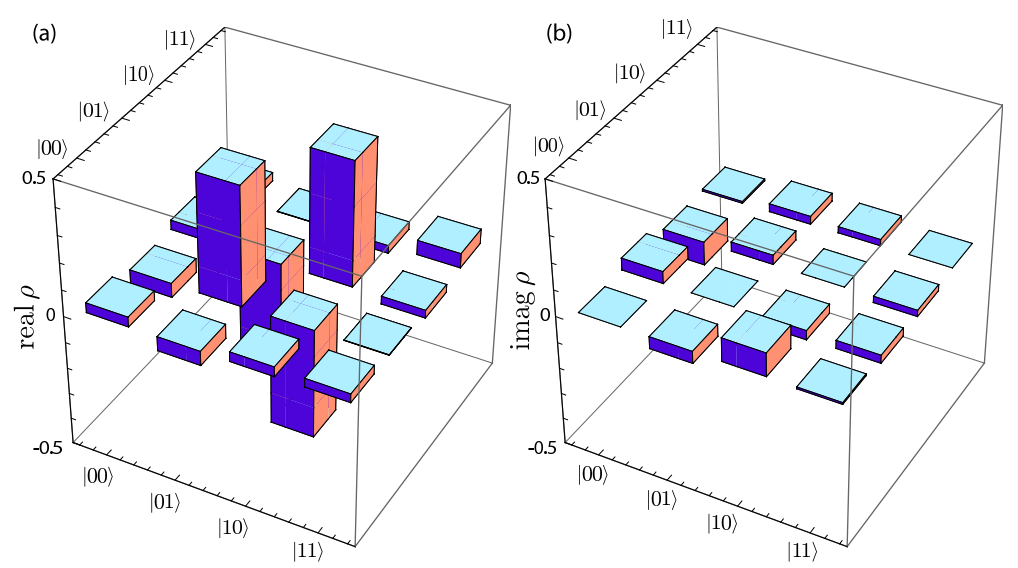

Figure 7.3: State tomography of $\left|\psi^{-}\right\rangle_{\text {ions }}$. The figure shows the real (a) and imaginary (b) elements of the reconstructed density matrix. The density matrix was obtained with a maximum likelihood method from 601 events measured in 9 different bases. From this density matrix we calculate an entangled state fidelity of $\mathcal{F}=0.87(2)$, a concurrence of $C=$ $0.77(4)$ and an entanglement of formation $E_{F}=0.69(6)$.

In addition to the fidelity measurements of Table 7.1, we also assess the generation of the maximally entangled antisymmetric Bell state $|\psi\rangle_{\text {ideal }}=1 / \sqrt{2}\left(|0\rangle_{a}|1\rangle_{b}-\right.$ $|1\rangle_{a}|0\rangle_{b}$ ) by full state tomography. The resulting density matrix shown in Fig. 7.3 is obtained using a maximum likelihood method [146]. From this density matrix we calculate an entangled state fidelity of $\mathcal{F}=0.87(2)$, a concurrence of $C=0.77(4)$ and an entanglement of formation $E_{F}=0.69(6)$ [143].

The observed entanglement and gate fidelity are consistent with known experimental errors. The primary contributions to the error are imperfect state detection (3\%), spatial mode mismatch on the beamsplitter (6\%), and detection of $\sigma$-polarized light due to the finite solid angle of collection and misalignment of the magnetic field $(<2 \%)$. Other sources, including imperfect state preparation, pulsed excitation to the wrong atomic state, dark counts of the PMT leading to false coincidence events, and multiple excitation due to pulsed laser light leakage, are each estimated to contribute to the overall error by much less than $1 \%$. Micromotion at the rf-drive frequency of the ion trap, which alters the spectrum of the emitted photons and can 
degrade the quantum interference, is expected to contribute to the overall error by less than $1 \%$.

\subsection{Success probability}

The quantum gate is a heralded, probabilistic process. The net probability for coincident detection of two emitted photons is given by:

$$
P_{\text {gate }}=\vartheta\left[p_{\pi} \eta T_{\text {fiber }} T_{\text {optics }} \xi(\Delta \Omega / 4 \pi)\right]^{2} \approx \vartheta\left(8.5 \times 10^{-8}\right)
$$

where $p_{\pi}=0.5$ is the fraction of photons with the correct polarization (half are filtered out as being produced by $\sigma$ decays); $\eta=0.15$ is the quantum efficiency of each PMT; $T_{\text {fiber }}=0.2$ is the coupling and transmission of each photon through the single-mode optical fiber; $T_{\text {optics }}=0.95$ is the transmission of each photon through the other optical components; $\xi=1-0.005=0.995$, where 0.005 is the branching ratio into the ${ }^{2} D_{3 / 2}$ level; and $\Delta \Omega / 4 \pi=0.02$ is the solid angle of light collection.

In the current setup, the attempt rate was limited to about $75 \mathrm{kHz}$, due the time required for the state preparation microwave pulse. This resulted in about one successful gate operation every $12 \mathrm{~min}$. However, the expression for $P_{\text {gate }}$ reveals multiple ways to substantially increase the success rate. The most dramatic increase would be achieved by increasing the effective solid angle of collection, which, for instance, could be accomplished by surrounding each ion with an optical cavity. Although improvements that increase the success probability of the gate operation can enhance scalability, even with a low success probability this gate can still be scaled to more complex systems [66]. 


\section{CHAPTER VIII}

\section{Teleportation}

"One of the problems has to do with the speed of light and the difficulties involved in trying to exceed it. You can't. Nothing travels faster than the speed of light with the possible exception of bad news, which obeys its own special laws."

-Douglas Adams, Mostly Harmless

Quantum teleportation is the faithful transfer of quantum states between systems that relies on the prior establishment of entanglement, but uses only classical communication during the transmission [7]. Teleportation, where quantum information is transferred between two disparate locations without traversing the space between the systems, is a stark realization of the counter-intuitive aspects of quantum physics. In addition, the ability to teleport quantum information is an essential ingredient for the long-distance quantum communication afforded by quantum repeaters [27] and may be a vital component to quantum computation [147].

Experimentally, teleportation has been accomplished in optical systems by using photons from spontaneous parametric down-conversion $[148,149]$ and squeezed light with continuous variable entanglement [150]. Teleportation has also been realized between photons and a single atomic ensemble $[151,152]$. Since photons are able to carry quantum information and establish entanglement over long distances, these experiments demonstrated the nonlocal behavior of teleportation. However, a quantum memory is required at both transmitting and receiving sites in order to scale 
this protocol to quantum networks and propagate quantum information over multiple nodes [28].

Deterministic teleportation between quantum memories has been implemented experimentally using trapped atomic ions in close proximity to one another, relying on the mutual Coulomb interaction to execute the necessary gate operations $[153$, $154,155]$. In contrast to the optical systems, these realizations feature long-lived coherences stored in good quantum memories but lack the ability to easily transmit quantum information over long distances.

In this chapter, we present the implementation of a heralded teleportation protocol where the advantages from both optical systems and quantum memories are combined to teleport quantum states between two trapped ytterbium ion qubits over a distance of about one meter [156]. Tomography is performed on the teleported states to fully characterize the system, and allow for complete process tomography of the protocol. After performing the protocol on a complete set of mutually unbiased basis states, we measure an average teleportation fidelity of $90(2) \%$. The fidelity of the operation is well above the $2 / 3$ threshold that could be achieved classically, unequivocally demonstrating the quantum nature of the process $[157,158]$.

\subsection{Teleportation protocol}

The experimental setup for the teleportation is identical to the one used to demonstrate the quantum gate (Fig. 7.1). A single ${ }^{171} \mathrm{Yb}^{+}$atom is confined and cooled in each of two nearly-identical traps separated by a distance of about one meter. The atoms are initialized to the state $|0\rangle$ by optical pumping via a $1 \mu$ s pulse of $369.5 \mathrm{~nm}$ 
light. We then prepare the ion $a$ and $b$ in the states:

$$
\begin{aligned}
|\psi\rangle_{a} & =\alpha|0\rangle_{a}+\beta|1\rangle_{a} \\
|\psi\rangle_{b} & =\frac{1}{\sqrt{2}}\left(|0\rangle_{b}+|1\rangle_{b}\right)
\end{aligned}
$$

by applying a resonant microwave pulse of controlled phase and duration $(0-16 \mu \mathrm{s})$ directly to one of the trap electrodes, where $|\alpha|^{2}+|\beta|^{2}=1$. The quantum state written into ion $a$ is the information we seek to teleport. While in the present case the amplitudes are determined by the applied microwave radiation, in principle $\alpha$ and $\beta$ could be unknown.

Following state preparation, each atom is excited by a 1 ps pulse of $\pi$-polarized light at $369.5 \mathrm{~nm}$, and this broadband excitation coherently drives the population in the hyperfine levels of the ${ }^{2} S_{1 / 2}$ state to complementary levels in the ${ }^{2} P_{1 / 2}$ state, as shown previously (Fig. 7.2). As each atom decays back to the ${ }^{2} S_{1 / 2}$ levels, it emits a single photon at $369.5 \mathrm{~nm}$. Considering only $\pi$ decays results in the frequency of the emitted photon becoming entangled with the electronic state of the ion, such that:

$$
\begin{aligned}
|\psi\rangle_{a} & =\alpha|0\rangle_{a}\left|\nu_{\text {blue }}\right\rangle_{a}+\beta|1\rangle_{a}\left|\nu_{\text {red }}\right\rangle_{a} \\
|\psi\rangle_{b} & =\frac{1}{\sqrt{2}}\left(|0\rangle_{b}\left|\nu_{\text {blue }}\right\rangle_{b}+|1\rangle_{b}\left|\nu_{\text {red }}\right\rangle_{b}\right)
\end{aligned}
$$

Spontaneously emitted photons are collected by an objective lens, coupled into a single-mode fiber, and directed to interfere at a 50:50 non-polarizing beamsplitter. As shown in Chap. VII, due to the quantum interference of the photons at the beamsplitter, a simultaneous detection at both output ports of the beamsplitter occurs only if the photons are in the $\left|\psi^{-}\right\rangle_{p h}$ state, so that the action of this heralded quantum gate is to project the ions into the entangled state:

$$
|\psi\rangle_{i o n s}={ }_{p h}\left\langle\psi^{-}\left|\left(|\psi\rangle_{a} \otimes|\psi\rangle_{b}\right)=\alpha\right| 0\right\rangle_{a}|1\rangle_{b}-\beta|1\rangle_{a}|0\rangle_{b}
$$


Even though the gate is a probabilistic process, we do not require postselection because the coicident detection of the two photons is a heralding event that announces the success of the quantum gate. In the teleportation protocol $\vartheta=1 / 4$ in Eq. 7.10 , so that the overall success probability for any $\alpha$ and $\beta$ is about $2.2 \times 10^{-8}$, limited by the efficiency of collecting and detecting both spontaneously emitted photons. Therefore, the previous steps (state preparation and pulsed excitation) are repeated at a rate of 40 to $75 \mathrm{kHz}$, including intermittent cooling, until the gate operation is successful (once every $12 \mathrm{~min}$, on average). As each attempt is independent of all others, this protocol allows for a sequence of unknown and unrelated input states.

After the success of the quantum gate has been confirmed by the heralding event, ion $a$ is subjected to another pulse of microwaves to execute the rotation $R_{y}(\pi / 2)$ (Sec. 4.5.4). The microwave rotation transforms the state given in Eq. 8.3 to:

$$
\begin{aligned}
|\psi\rangle_{\text {ions }} & =\frac{\alpha}{\sqrt{2}}\left(|0\rangle_{a}+|1\rangle_{a}\right)|1\rangle_{b}-\frac{\beta}{\sqrt{2}}\left(-|0\rangle_{a}+|1\rangle_{a}\right)|0\rangle_{b} \\
& =\frac{1}{\sqrt{2}}\left(\alpha|1\rangle_{b}+\beta|0\rangle_{b}\right)|0\rangle_{a}+\frac{1}{\sqrt{2}}\left(\alpha|1\rangle_{b}-\beta|0\rangle_{b}\right)|1\rangle_{a}
\end{aligned}
$$

We then measure ion $a$ using the state dependent fluorescence technique discussed in Chap. IV. As is apparent from Eq. 8.4, measuring ion $a$ projects ion $b$ into one of the two states:

$$
\begin{aligned}
& \text { If measured }|0\rangle_{a} \Rightarrow|\psi\rangle_{b}=\alpha|1\rangle_{b}+\beta|0\rangle_{b} \\
& \text { If measured }|1\rangle_{a} \Rightarrow|\psi\rangle_{b}=\alpha|1\rangle_{b}-\beta|0\rangle_{b}
\end{aligned}
$$

The result of the measurement on ion $a$ is relayed through a classical communication channel and used to determine the necessary phase of a conditional microwave $\pi$ pulse applied to ion $b$ to recover the state initially written to ion $a$. If $|0\rangle_{a}$ is measured, the rotation $R_{x}(\pi)$ is applied to ion $b$ so that:

$$
R_{x}(\pi)\left(\alpha|1\rangle_{b}+\beta|0\rangle_{b}\right)=\alpha|0\rangle_{b}+\beta|1\rangle_{b}
$$


On the other hand, if $|1\rangle_{a}$ is measured, the rotation $R_{y}(\pi)$ is applied to ion $b$ :

$$
R_{y}(\pi)\left(\alpha|1\rangle_{b}-\beta|0\rangle_{b}\right)=\alpha|0\rangle_{b}+\beta|1\rangle_{b}
$$

where we have ignored any global phase factors. Thus, the state amplitudes initially written to ion $a$ are now stored in ion $b$, which completes the teleportation of the quantum state between the two distant matter qubits.

\subsection{Experimental evaluation}

We execute on the teleportation protocol on a set of six mutually unbiased basis state $|\psi\rangle_{\text {ideal }} \in\{1 / \sqrt{2}(|0\rangle+|1\rangle), 1 / \sqrt{2}(|0\rangle-|1\rangle), 1 / \sqrt{2}(|0\rangle+i|1\rangle), 1 / \sqrt{2}(|0\rangle-$ $i|1\rangle),|0\rangle,|1\rangle\}$, and evaluate the process by performing state tomography on each teleported state. A single-qubit density matrix can be reconstructed by measuring the state in three mutually unbiased measurement bases. Of course, measurement of the ion occurs via the aforementioned state fluorescence technique, and therefore only distinguishes between $|0\rangle$ and $|1\rangle$ (z-basis); two states such as $1 / \sqrt{2}(|0\rangle+|1\rangle)$ and $1 / \sqrt{2}(|0\rangle-|1\rangle)(x$-basis) are not distinguishable by fluorescence alone. Measurement in the remaining two bases requires an additional microwave pulse before detection. We define the rotation $\left\{R_{y}(\pi / 2), R_{x}(\pi / 2), R(0)\right\}$ before detection to correspond to measurement in the basis $\{x, y, z\}$. These measurements allow reconstruction of the single-qubit density matrix, $\rho$, for each teleported state using a simple analytical expression [159], with the results shown in Fig. 8.1. The fidelity of the teleportation protocol, defined as the overlap of the ideal and measured density matrices $\mathcal{F}=\operatorname{tr}\left(\rho_{\text {ideal }} \rho\right)={ }_{\text {ideal }}\langle\psi|\rho| \psi\rangle_{\text {ideal }}$, for this set states is measured to be $\mathcal{F}=\{0.91(3), 0.88(4), 0.92(4), 0.91(4), 0.93(4), 0.88(4)\}$. This yields an average teleportation fidelity of $\overline{\mathcal{F}}=0.90(2)$ [156].

The reconstructed density matrices also facilitate full characterization of the tele- 


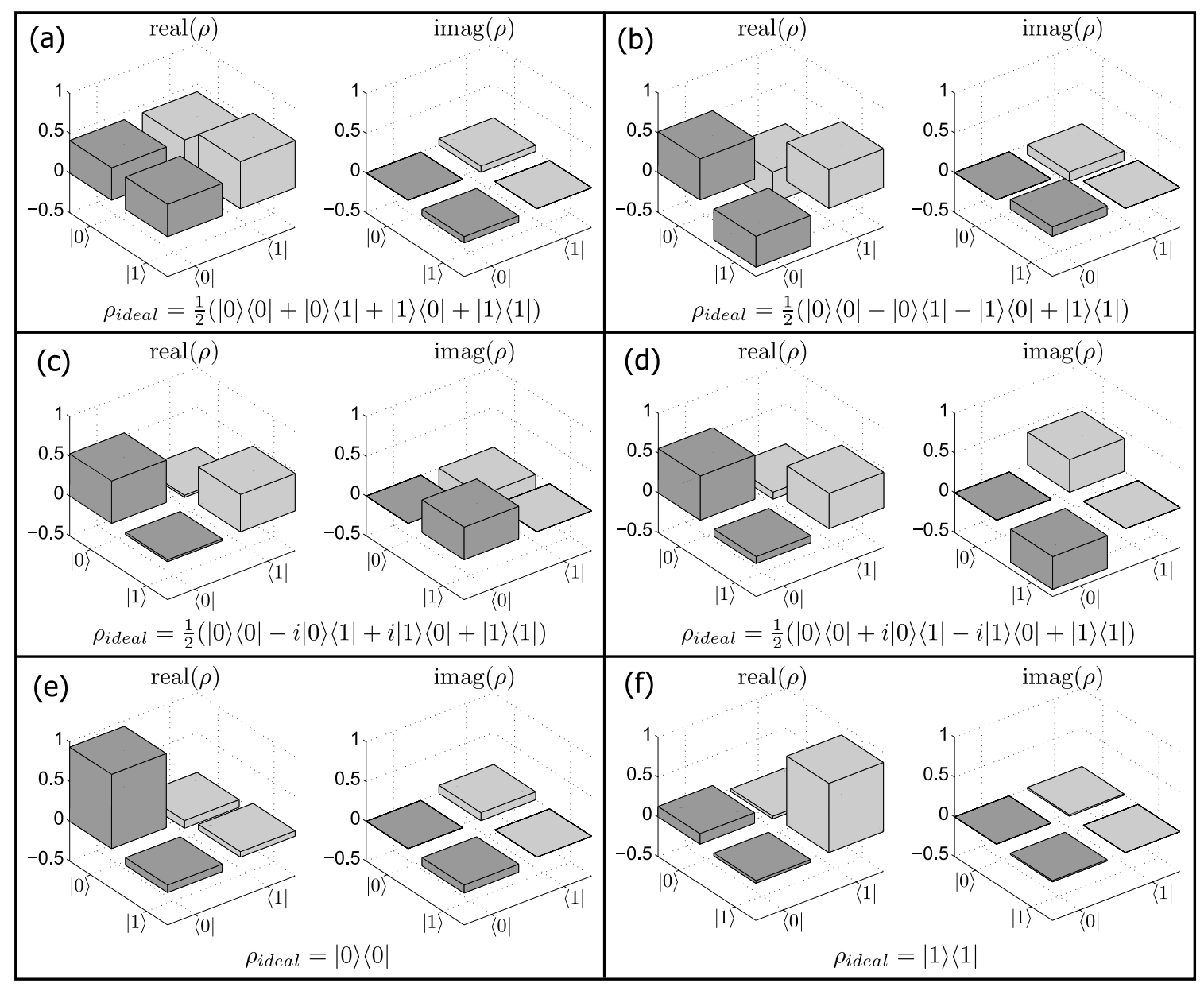

Figure 8.1: Tomography of the teleported quantum states. The reconstructed density matrices, $\rho$, for the six unbiased basis states teleported from ion $a$ to ion $b$ : (a) $|\psi\rangle_{\text {ideal }}=$ $1 / \sqrt{2}(|0\rangle+|1\rangle)$ teleported with fidelity $\mathcal{F}=0.91(3)$, (b) $|\psi\rangle_{\text {ideal }}=1 / \sqrt{2}(|0\rangle-|1\rangle)$ teleported with fidelity $\mathcal{F}=0.88(4)$, (c) $|\psi\rangle_{\text {ideal }}=1 / \sqrt{2}(|0\rangle+i|1\rangle)$ teleported with fidelity $\mathcal{F}=0.92(4),(\mathrm{d})|\psi\rangle_{\text {ideal }}=1 / \sqrt{2}(|0\rangle-i|1\rangle)$ teleported with fidelity $\mathcal{F}=0.91(4)$, (e) $|\psi\rangle_{\text {ideal }}=|0\rangle$ teleported with fidelity $\mathcal{F}=0.93(4)$, and (f) $|\psi\rangle_{\text {ideal }}=|1\rangle$ teleported with fidelity $\mathcal{F}=0.88(4)$. These measurements yield an average teleportation fidelity $\overline{\mathcal{F}}=0.90(2)$. The data shown comprise a total of 1285 events in 253 hours. 
portation protocol by quantum process tomography. We can completely describe the effect of the teleportation protocol on an input state $\rho_{i n}$ by determining the process matrix $\chi$, defined by [31]:

$$
\rho=\sum_{l, k=0}^{3} \chi_{l k} \hat{\sigma}_{l} \rho_{i n} \hat{\sigma}_{k}
$$

where to evaluate our process we assume $\rho_{i n}=|\psi\rangle_{\text {ideal }}\langle\psi|$. For the teleportation protocol, the ideal process matrix, $\chi_{\text {ideal }}$, has only one nonzero component, $\left(\chi_{\text {ideal }}\right)_{00}=1$, corresponding to the identity operation on the input state. The experimentally determined the process matrix $\chi$, presented in Fig. 8.2, was reconstructed using a maximum likelihood method [160]. Given this process matrix, we calculate the process fidelity to be $\mathcal{F}_{\text {process }}=\operatorname{tr}\left(\chi_{\text {ideal }} \chi\right)=0.84(2)$ [156]. Since the average fidelity and process fidelity are related by $\mathcal{F}_{\text {process }}=(3 \overline{\mathcal{F}}-1) / 2$, this is consistent with the average fidelity found above [161].

\subsection{Error analysis}

The experimental errors in the teleportation protocol differ slightly from just the implementation of the quantum gate, due to the additional single-qubit operations involved. The primary sources of error that reduce the average fidelity are photon mode mismatch at the 50:50 beamsplitter (4\%), imperfect state detection (3.5\%), and polarization-mixing resulting from the nonzero numerical aperture of the objective lens and from misalignment with respect to the magnetic field $(2 \%)$. Other sources, including incomplete state preparation, pulsed excitation to the wrong atomic state, dark counts of the PMT leading to false coincidence events, photon polarization rotation while traversing the optical fiber, and multiple excitation resulting from pulsed laser light leakage, are each expected to contribute to the error by much less than $1 \%$. Residual micromotion at the rf-drive frequency of the ion trap, which alters 


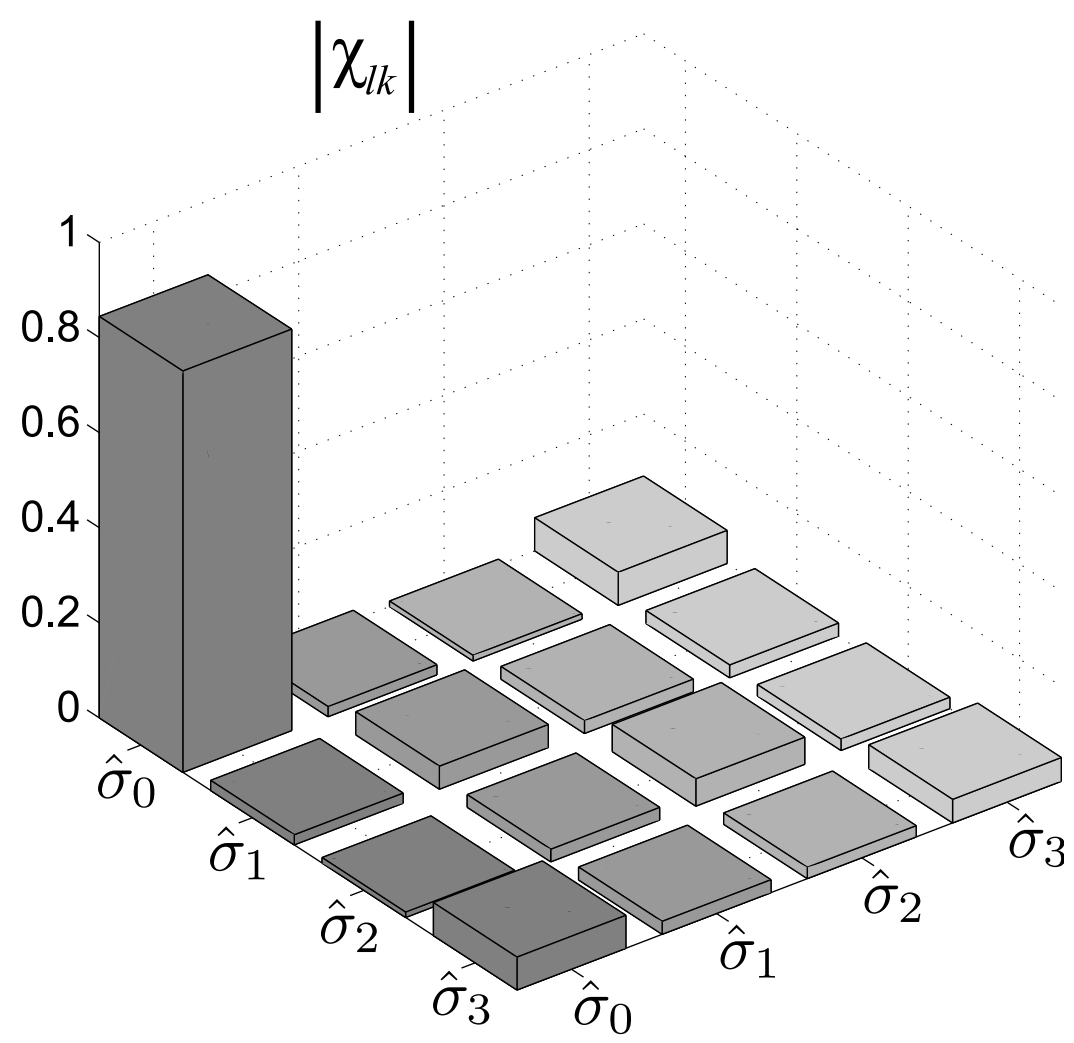

Figure 8.2: Process tomography of the teleportation protocol. Displayed in the figure is the absolute value of the components of the reconstructed process matrix, $\left|\chi_{l k}\right|$, with $l, k=0,1,2,3$. The process matrix is reconstructed from the state tomography data (Fig. 8.1) using a maximum likelihood method. The elements are given in terms of the Pauli operators (Chap. II). As intended, the dominant component of $\chi$ is the contribution of the identity operation, yielding an overall process fidelity $\mathcal{F}_{\text {process }}=\operatorname{tr}\left(\chi_{\text {ideal }} \chi\right)=0.84(2)$, consistent with the average fidelity cited above. 
the spectrum of the emitted photons and degrades the quantum interference, reduces the average fidelity by less than $1 \%$.

A detailed calculation of the two most prominent error contributions are presented below.

8.3.1 Photon spatial mode-mismatch at the beamsplitter We determine the effect of imperfect spatial mode-overlap of two photons incident on the beamsplitter by utilizing the formalism presented in Chap. V. In this case, though, we will only consider two photons impinging on the beamsplitter, rather than the train of photons considered previously; in the experiment, we know we are looking for coincident detections and that photon from different excitation pulses are well-separated in time, so we can make this simplification with loss of generality.

First, we calculate the effect of the electric field operators on the four possible input states $\left|\nu_{\text {red }}\right\rangle_{a}\left|\nu_{\text {red }}\right\rangle_{b},\left|\nu_{\text {red }}\right\rangle_{a}\left|\nu_{\text {blue }}\right\rangle_{b},\left|\nu_{b l u e}\right\rangle_{a}\left|\nu_{\text {red }}\right\rangle_{b}$, and $\left|\nu_{b l u e}\right\rangle_{a}\left|\nu_{b l u e}\right\rangle_{b}$. As an attempt to unclutter the expressions a litte, we will also shift our notation slightly. Hence forth we will not label the states with $a$ or $b$ unless there are possible ambiguities. Instead, we will write, e.g. $\left|\nu_{\text {red }}\right\rangle_{a}\left|\nu_{b l u e}\right\rangle_{b}=|R B\rangle$, where the ordering of the frequencies determine to which subsystem they apply (e.g. in this case "R" indicates a "red" photon as part of system $a$ ).

The four possible ways to get a photon in each exit port of the beamsplitter are:

$$
E_{3 b}^{+} E_{4 b}^{+}\left(t_{d}\right), E_{3 b}^{+} E_{4 a}^{+}\left(t_{d}\right), E_{3 a}^{+} E_{4 b}^{+}\left(t_{d}\right), E_{3 a}^{+} E_{4 a}^{+}\left(t_{d}\right)
$$

where the electric field operators are defined as in Eq. 5.5, with $\hat{b}^{\dagger}$ and $\hat{a}^{\dagger}$ as the creation operators for a $\left|\nu_{\text {blue }}\right\rangle=|B\rangle$ and $\left|\nu_{\text {red }}\right\rangle=|R\rangle$ photon, respectively (as in Chap. VII). In the subsequent calculation, though, we will suppress the exponential decay factor, the Heaviside step function, and the normalization terms of Eq. 5.5. Presently, we are concerned only with the resulting density matrix if a coincident 
detection of two photons occurs, and thus in this derivation these other factors can just be regarded as part of a normalization term.

There are then 16 terms to calcuate; fortunately, many of them have an identical form.

$$
\begin{aligned}
E_{3 b}^{+} E_{4 b}^{+}\left(t_{d}\right)|R R\rangle= & \frac{1}{2}\left[\xi_{1} \hat{a}_{1}+\xi_{2} \hat{a}_{2}\right]\left[-\xi_{1}\left(t_{d}\right) \hat{a}_{1}+\xi_{2}\left(t_{d}\right) \hat{a}_{2}\right] \hat{a}_{1}^{\dagger} \hat{a}_{2}^{\dagger}|0\rangle \\
= & \frac{1}{2}\left[-\xi_{1} \xi_{1}\left(t_{d}\right)\left(\hat{a}_{1}\right)^{2}-\xi_{2} \xi_{1}\left(t_{d}\right) \hat{a}_{2} \hat{a}_{1}\right. \\
& \left.+\xi_{1} \xi_{2}\left(t_{d}\right) \hat{a}_{1} \hat{a}_{2}+\xi_{2} \xi_{2}\left(t_{d}\right)\left(\hat{a}_{2}\right)^{2}\right] \hat{a}_{1}^{\dagger} \hat{a}_{2}^{\dagger}|0\rangle \\
= & \frac{1}{2}\left[\xi_{1} \xi_{2}\left(t_{d}\right)-\xi_{2} \xi_{1}\left(t_{d}\right)\right]|0\rangle
\end{aligned}
$$

This will also be the result for $E_{3 a}^{+} E_{4 a}^{+}\left(t_{d}\right)|B B\rangle$, as can be seen by switching $\hat{a}$ with $\hat{b}$, and $|R\rangle$ with $|B\rangle$.

Next, we calculate:

$$
E_{3 b}^{+} E_{4 b}^{+}\left(t_{d}\right)|R R\rangle=\frac{1}{2}\left[\xi_{1} \hat{b}_{1}+\xi_{2} \hat{b}_{2}\right]\left[-\xi_{1}\left(t_{d}\right) \hat{b}_{1}+\xi_{2}\left(t_{d}\right) \hat{b}_{2}\right] \hat{a}_{1}^{\dagger} \hat{a}_{2}^{\dagger}|0\rangle=0
$$

because $\hat{b} \hat{a}^{\dagger}|0\rangle=0$. Of course, the term will vanish whenever there are more annihilation operators of a specific mode than creation operators. As such, the following terms also vanish:

$$
\begin{aligned}
0 & =E_{3 a}^{+} E_{4 a}^{+}\left(t_{d}\right)|B B\rangle=E_{3 a}^{+} E_{4 b}^{+}\left(t_{d}\right)|B B\rangle=E_{3 a}^{+} E_{4 b}^{+}\left(t_{d}\right)|R R\rangle \\
& =E_{3 b}^{+} E_{4 a}^{+}\left(t_{d}\right)|B B\rangle=E_{3 b}^{+} E_{4 a}^{+}\left(t_{d}\right)|R R\rangle=E_{3 a}^{+} E_{4 a}^{+}\left(t_{d}\right)|R B\rangle \\
& =E_{3 a}^{+} E_{4 a}^{+}\left(t_{d}\right)|B R\rangle=E_{3 b}^{+} E_{4 b}^{+}\left(t_{d}\right)|B R\rangle=E_{3 b}^{+} E_{4 b}^{+}\left(t_{d}\right)|R B\rangle
\end{aligned}
$$

The last combinations we need to calculate yield:

$$
\begin{aligned}
E_{3 a}^{+} E_{4 b}^{+}\left(t_{d}\right)|R B\rangle= & \frac{1}{2}\left[\xi_{1} \hat{a}_{1}+\xi_{2} \hat{a}_{2}\right]\left[-\xi_{1}\left(t_{d}\right) \hat{b}_{1}+\xi_{2}\left(t_{d}\right) \hat{b}_{2}\right] \hat{a}_{1}^{\dagger} \hat{b}_{2}^{\dagger}|0\rangle \\
= & \frac{1}{2}\left[-\xi_{1} \xi_{1}\left(t_{d}\right) \hat{a}_{1} \hat{b}_{1}-\xi_{2} \xi_{1}\left(t_{d}\right) \hat{a}_{2} \hat{b}_{1}\right. \\
& \left.+\xi_{1} \xi_{2}\left(t_{d}\right) \hat{a}_{1} \hat{b}_{2}+\xi_{2} \xi_{2}\left(t_{d}\right) \hat{a}_{2} \hat{b}_{2}\right] \hat{a}_{1}^{\dagger} \hat{b}_{2}^{\dagger}|0\rangle \\
= & \frac{1}{2} \xi_{1} \xi_{2}\left(t_{d}\right)
\end{aligned}
$$


The same result will result from $E_{3 b}^{+} E_{4 a}^{+}\left(t_{d}\right)|B R\rangle$. We also calculate:

$$
\begin{aligned}
E_{3 a}^{+} E_{4 b}^{+}\left(t_{d}\right)|B R\rangle= & \frac{1}{2}\left[\xi_{1} \hat{a}_{1}+\xi_{2} \hat{a}_{2}\right]\left[-\xi_{1}\left(t_{d}\right) \hat{b}_{1}+\xi_{2}\left(t_{d}\right) \hat{b}_{2}\right] \hat{b}_{1}^{\dagger} \hat{a}_{2}^{\dagger}|0\rangle \\
= & \frac{1}{2}\left[-\xi_{1} \xi_{1}\left(t_{d}\right) \hat{a}_{1} \hat{b}_{1}-\xi_{2} \xi_{1}\left(t_{d}\right) \hat{a}_{2} \hat{b}_{1}\right. \\
& \left.+\xi_{1} \xi_{2}\left(t_{d}\right) \hat{a}_{1} \hat{b}_{2}+\xi_{2} \xi_{2}\left(t_{d}\right) \hat{a}_{2} \hat{b}_{2}\right] \hat{b}_{1}^{\dagger} \hat{a}_{2}^{\dagger}|0\rangle \\
= & -\frac{1}{2} \xi_{2} \xi_{1}\left(t_{d}\right)
\end{aligned}
$$

The result of Eq. 8.12 will also be the result for the term $E_{3 b}^{+} E_{4 a}^{+}\left(t_{d}\right)|R B\rangle$.

Having calculated the terms we will need, we move on to actually looking at the two ion-photon system. As seen above, the system after excitation and spontaneous emission of a $\pi$-polarized photon is:

$$
|\psi\rangle_{i o n, p h}=\frac{1}{\sqrt{2}}[\alpha|00\rangle|B B\rangle+\beta|10\rangle|R B\rangle+\alpha|01\rangle|B R\rangle+\beta|11\rangle|R R\rangle]
$$

We want to know the density matrix of the two ions after a coincident detection of two photons. In the above calculations we calculated the sixteen (six non-vanishing) ways to get a coincident detection. Using terminology similar to that in Ref. [31], we will define the electric field measurement operator as:

$$
M_{p h, j k}=E_{3 j}^{+} E_{4 k}^{+}\left(t_{d}\right)
$$

where $j, k=a, b$. In our previous discussion of the joint detection probability in Chap. V, we wrote the probability of a coincident detection as:

$$
\begin{aligned}
P_{J} & ={ }_{p h}\left\langle\psi\left|E_{4 k}^{-}\left(t_{d}\right) E_{3 j}^{-} E_{3 j}^{+} E_{4 k}^{+}\left(t_{d}\right)\right| \psi\right\rangle_{p h} \\
& ={ }_{p h}\left\langle\psi\left|M_{p h, j k}^{\dagger} M_{p h, j k}\right| \psi\right\rangle_{p h} \\
& =\operatorname{tr}\left(M_{p h, j k}^{\dagger} M_{p h, j k}|\psi\rangle_{p h}\langle\psi|\right)
\end{aligned}
$$

where in the last two lines we have used our definition of the electric field measurement operator and the usual properties of the trace [31]. 
In this formalism, the density matrix of the two ions will be given by a partial trace over the photon states of the electric field operators acting on the two ion-photon system:

$$
\begin{aligned}
\rho_{\text {ions }}= & t_{p h}\left[\sum_{j, k} M_{p h, j k}^{\dagger} M_{p h, j k}|\psi\rangle_{i o n, p h}\langle\psi|\right] \\
= & \frac{1}{2}\left[|\alpha|^{2}|00\rangle\langle 00|\left\langle B B\left|M_{p h, j k}^{\dagger} M_{p h, j k}\right| B B\right\rangle\right. \\
& +\alpha \beta^{*}|00\rangle\left\langle\left. 10\left|\left\langle B B\left|M_{p h, j k}^{\dagger} M_{p h, j k}\right| R B\right\rangle+\right| \alpha\right|^{2} \mid 00\right\rangle\langle 01|\left\langle B B\left|M_{p h, j k}^{\dagger} M_{p h, j k}\right| B R\right\rangle \\
& +\alpha \beta^{*}|00\rangle\left\langle 11\left|\left\langle B B\left|M_{p h, j k}^{\dagger} M_{p h, j k}\right| R R\right\rangle+\beta \alpha^{*}\right| 10\right\rangle\langle 00|\left\langle R B\left|M_{p h, j k}^{\dagger} M_{p h, j k}\right| B B\right\rangle \\
& +|\beta|^{2}|10\rangle\left\langle 10\left|\left\langle R B\left|M_{p h, j k}^{\dagger} M_{p h, j k}\right| R B\right\rangle+\beta \alpha^{*}\right| 10\right\rangle\langle 01|\left\langle R B\left|M_{p h, j k}^{\dagger} M_{p h, j k}\right| B R\right\rangle \\
& +|\beta|^{2}|10\rangle\left\langle\left. 11\left|\left\langle R B\left|M_{p h, j k}^{\dagger} M_{p h, j k}\right| R R\right\rangle+\right| \alpha\right|^{2} \mid 01\right\rangle\langle 00|\left\langle B R\left|M_{p h, j k}^{\dagger} M_{p h, j k}\right| B B\right\rangle \\
& +\alpha \beta^{*}|01\rangle\left\langle\left. 10\left|\left\langle B R\left|M_{p h, j k}^{\dagger} M_{p h, j k}\right| R B\right\rangle+\right| \alpha\right|^{2} \mid 01\right\rangle\langle 01|\left\langle B R\left|M_{p h, j k}^{\dagger} M_{p h, j k}\right| B R\right\rangle \\
& +\alpha \beta^{*}|01\rangle\left\langle 11\left|\left\langle B R\left|M_{p h, j k}^{\dagger} M_{p h, j k}\right| R R\right\rangle+\beta \alpha^{*}\right| 11\right\rangle\langle 00|\left\langle R R\left|M_{p h, j k}^{\dagger} M_{p h, j k}\right| B B\right\rangle \\
& +|\beta|^{2}|11\rangle\left\langle 10\left|\left\langle R R\left|M_{p h, j k}^{\dagger} M_{p h, j k}\right| R B\right\rangle+\beta \alpha^{*}\right| 11\right\rangle\langle 01|\left\langle R R\left|M_{p h, j k}^{\dagger} M_{p h, j k}\right| B R\right\rangle \\
& \left.+|\beta|^{2}|11\rangle\langle 11|\left\langle R R\left|M_{p h, j k}^{\dagger} M_{p h, j k}\right| R R\right\rangle\right]
\end{aligned}
$$

where in the last several lines, the sum $\sum_{j, k}$ is implicit. Using our results for the action of the different measurement operators on the states, we then get:

$$
\begin{aligned}
\rho_{\text {ions }}= & \frac{1}{8}\left[| \alpha | ^ { 2 } | 0 0 \rangle \left\langle00|| \xi_{1} \xi_{2}\left(t_{d}\right)-\left.\xi_{2} \xi_{1}\left(t_{d}\right)\right|^{2}+0+0+0+0\right.\right. \\
& +|\beta|^{2}|10\rangle\langle 10|\left(\left|\xi_{1} \xi_{2}\left(t_{d}\right)\right|^{2}+\left|\xi_{2} \xi_{1}\left(t_{d}\right)\right|^{2}\right) \\
& +\beta \alpha^{*}|10\rangle\langle 01|\left(-\xi_{1}^{*} \xi_{2}^{*}\left(t_{d}\right) \xi_{2} \xi_{1}\left(t_{d}\right)-\xi_{1} \xi_{2}\left(t_{d}\right) \xi_{2}^{*} \xi_{1}^{*}\left(t_{d}\right)\right)+0 \\
& +0+\alpha \beta^{*}|01\rangle\langle 10|\left(-\xi_{1} \xi_{2}\left(t_{d}\right) \xi_{2}^{*} \xi_{1}^{*}\left(t_{d}\right)-\xi_{1}^{*} \xi_{2}^{*}\left(t_{d}\right) \xi_{2} \xi_{1}\left(t_{d}\right)\right) \\
& |\alpha|^{2}|01\rangle\langle 01|\left(\left|\xi_{2} \xi_{1}\left(t_{d}\right)\right|^{2}+\left|\xi_{1} \xi_{2}\left(t_{d}\right)\right|^{2}\right)+0+0 \\
& +0+0+|\beta|^{2}|11\rangle\left\langle 11|| \xi_{1} \xi_{2}\left(t_{d}\right)-\left.\xi_{2} \xi_{1}\left(t_{d}\right)\right|^{2}\right]
\end{aligned}
$$

After a coincident detection, the next step in the teleportation protocol was to 
rotate ion $a$ with a $R_{y}(\pi / 2)$ microwave pulse, and then measure it. The ion-ion density matrix after rotation of ion $a$ is:

$$
\begin{aligned}
\left(R_{y}(\pi / 2)\right)_{a} \rho_{\text {ions }} & \left(R_{y}^{\dagger}(\pi / 2)\right)_{a} \\
=\quad & \frac{1}{16}\left[|\alpha|^{2}\left|\xi_{1} \xi_{2}\left(t_{d}\right)-\xi_{2} \xi_{1}\left(t_{d}\right)\right|^{2}(|0\rangle+|1\rangle)|0\rangle(\langle 0|+\langle 1|)\langle 0|\right. \\
& +|\beta|^{2}\left(\left|\xi_{1} \xi_{2}\left(t_{d}\right)\right|^{2}+\left|\xi_{2} \xi_{1}\left(t_{d}\right)\right|^{2}\right)(-|0\rangle+|1\rangle)|0\rangle(-\langle 0|+\langle 1|)\langle 0| \\
& +\beta \alpha^{*}\left(-\xi_{1}^{*} \xi_{2}^{*}\left(t_{d}\right) \xi_{2} \xi_{1}\left(t_{d}\right)-\xi_{1} \xi_{2}\left(t_{d}\right) \xi_{2}^{*} \xi_{1}^{*}\left(t_{d}\right)\right) \\
& \times(-|0\rangle+|1\rangle)|0\rangle(\langle 0|+\langle 1|)\langle 1| \\
& +\alpha \beta^{*}\left(-\xi_{1} \xi_{2}\left(t_{d}\right) \xi_{2}^{*} \xi_{1}^{*}\left(t_{d}\right)-\xi_{1}^{*} \xi_{2}^{*}\left(t_{d}\right) \xi_{2} \xi_{1}\left(t_{d}\right)\right) \\
& \times(|0\rangle+|1\rangle)|1\rangle(-\langle 0|+\langle 1|)\langle 0| \\
& +|\alpha|^{2}\left(\left|\xi_{2} \xi_{1}\left(t_{d}\right)\right|^{2}+\left|\xi_{1} \xi_{2}\left(t_{d}\right)\right|^{2}\right)(|0\rangle+|1\rangle)|1\rangle(\langle 0|+\langle 1|)\langle 1| \\
& \left.|\beta|^{2}\left|\xi_{1} \xi_{2}\left(t_{d}\right)-\xi_{2} \xi_{1}\left(t_{d}\right)\right|^{2}(-|0\rangle+|1\rangle)|1\rangle(-\langle 0|+\langle 1|)\langle 1|\right] \quad(8.18)
\end{aligned}
$$

Now we measure ion $a$. Analogous to the electric field measurement operator, we will define an operator for the measurement of the quantum state of the ion. At the moment, we will assume ideal measurement of the ion. The influence of imperfect state detection on the fidelity is calculated in the next section; as both are independently small, it is a good approximation to consider each separately.

Define our (ideal) measurement operators for the ion state to be [31]:

$$
M_{0 j}=|0\rangle_{j}\left\langle 0\left|, M_{1 j}=\right| 1\right\rangle_{j}\langle 1|
$$

where here $j=a, b$ denotes operation on the $j$ th ion. Since with the final rotation on ion $b$, conditioned on the measurement of ion $a$, the states will be equivalent, we only work out the case for $|0\rangle_{a}$ below. Measurement of ion $a$ is defined similarly to the case for the photons, where the density matrix of ion $b$ is given by the partial 
trace of $a$ on the measurment operator and the quantum state of the ions. We get:

$$
\begin{aligned}
\rho_{b}= & \operatorname{tr}_{a}\left(M_{0 a}^{\dagger} M_{0 a}\left(R_{y}(\pi / 2)\right)_{a} \rho_{\text {ions }}\left(R_{y}^{\dagger}(\pi / 2)\right)_{a}\right) \\
= & \frac{1}{16}\left[\left(|\alpha|^{2}\left|\xi_{1} \xi_{2}\left(t_{d}\right)-\xi_{2} \xi_{1}\left(t_{d}\right)\right|^{2}+|\beta|^{2}\left(\left|\xi_{1} \xi_{2}\left(t_{d}\right)\right|^{2}+\left|\xi_{2} \xi_{1}\left(t_{d}\right)\right|^{2}\right)\right)|0\rangle\langle 0|\right. \\
& -\beta \alpha^{*}\left(-\xi_{1}^{*} \xi_{2}^{*}\left(t_{d}\right) \xi_{2} \xi_{1}\left(t_{d}\right)-\xi_{1} \xi_{2}\left(t_{d}\right) \xi_{2}^{*} \xi_{1}^{*}\left(t_{d}\right)\right)|0\rangle\langle 1| \\
& -\alpha \beta^{*}\left(-\xi_{1} \xi_{2}\left(t_{d}\right) \xi_{2}^{*} \xi_{1}^{*}\left(t_{d}\right)-\xi_{1}^{*} \xi_{2}^{*}\left(t_{d}\right) \xi_{2} \xi_{1}\left(t_{d}\right)\right)|1\rangle\langle 0| \\
& \left.+\left(|\alpha|^{2}\left(\left|\xi_{2} \xi_{1}\left(t_{d}\right)\right|^{2}+\left|\xi_{1} \xi_{2}\left(t_{d}\right)\right|^{2}\right)+|\beta|^{2}\left|\xi_{1} \xi_{2}\left(t_{d}\right)-\xi_{2} \xi_{1}\left(t_{d}\right)\right|^{2}\right)|1\rangle\langle 1|\right](8.2
\end{aligned}
$$

and recall that in the teleportation protocol, if $|0\rangle_{a}$ is measured then ion $b$ has a $R_{x}(\pi)$ microwave pulse applied to it. Since this just flips the state of the ion, the final density matrix for ion $b$ is given by:

$$
\begin{aligned}
\rho_{b}= & \frac{1}{16}\left[\left(|\alpha|^{2}\left(\left|\xi_{2} \xi_{1}\left(t_{d}\right)\right|^{2}+\left|\xi_{1} \xi_{2}\left(t_{d}\right)\right|^{2}\right)+|\beta|^{2}\left|\xi_{1} \xi_{2}\left(t_{d}\right)-\xi_{2} \xi_{1}\left(t_{d}\right)\right|^{2}\right)|0\rangle\langle 0|\right. \\
& -\alpha \beta^{*}\left(-\xi_{1} \xi_{2}\left(t_{d}\right) \xi_{2}^{*} \xi_{1}^{*}\left(t_{d}\right)-\xi_{1}^{*} \xi_{2}^{*}\left(t_{d}\right) \xi_{2} \xi_{1}\left(t_{d}\right)\right)|0\rangle\langle 1| \\
& -\beta \alpha^{*}\left(-\xi_{1}^{*} \xi_{2}^{*}\left(t_{d}\right) \xi_{2} \xi_{1}\left(t_{d}\right)-\xi_{1} \xi_{2}\left(t_{d}\right) \xi_{2}^{*} \xi_{1}^{*}\left(t_{d}\right)\right)|1\rangle\langle 0| \\
& \left.+\left(|\alpha|^{2}\left|\xi_{1} \xi_{2}\left(t_{d}\right)-\xi_{2} \xi_{1}\left(t_{d}\right)\right|^{2}+|\beta|^{2}\left(\left|\xi_{1} \xi_{2}\left(t_{d}\right)\right|^{2}+\left|\xi_{2} \xi_{1}\left(t_{d}\right)\right|^{2}\right)\right)|1\rangle\langle 1|\right](8
\end{aligned}
$$

In order to make sense of this, we need to determine the spatial mode factors $\xi$ in terms of a measured quantity in the lab. The quantity we measure is the visibility of the interferometer. The visibility is defined as:

$$
V=\frac{I_{\max }-I_{\min }}{I_{\max }+I_{\min }}
$$

where $I_{\max }$ and $I_{\min }$ are the maxium and minimum intensities of the incident light. If two fields are incident, with amplitudes $E_{1}$ and $E_{2}$, then we know $I_{\max }=\left|E_{1}+E_{2}\right|^{2}$ and $I_{\max }=\left|E_{1}-E_{2}\right|^{2}$. On the other hand, the intensity of a single field is just $I_{1}=\left|E_{1}\right|^{2}$

Suppose now that we have a function $e(r)$ that describes the amplitude of the incident light as a function of position. However, the detector measures the intensity 
$I$ incident over the area of the detector. If we assume the detector area completely covers the incident mode, then the registered intensity will be the integral over space:

$$
I_{e}=\int|e(r)|^{2} d r
$$

If two fields are incident, $e(r)$ and $f(r)$, then we can define the maximum and minimum intensities as:

$$
\begin{aligned}
I_{\text {max }} & =\int|e(r)+f(r)|^{2} d r \\
I_{\text {min }} & =\int|e(r)-f(r)|^{2} d r
\end{aligned}
$$

and thus,

$$
\begin{aligned}
I_{\text {max }} & =\int(e(r)+f(r))\left(e^{*}(r)+f^{*}(r)\right) d r \\
& =\int\left(|e(r)|^{2}+|f(r)|^{2}+f(r) e^{*}(r)+e(r) f^{*}(r)\right) d r \\
& =I_{e}+I_{f}+\int\left(f(r) e^{*}(r)+e(r) f^{*}(r)\right) d r
\end{aligned}
$$

Similarly,

$$
I_{\text {min }}=I_{e}+I_{f}-\int\left(f(r) e^{*}(r)+e(r) f^{*}(r)\right) d r
$$

Therefore, we can write the visibility of the interferometer as:

$$
V=\frac{\int\left(f(r) e^{*}(r)+e(r) f^{*}(r)\right) d r}{I_{e}+I_{f}}
$$

Of course, we defined the relative phase between $e(r)$ and $f(r)$ when we defined the maximum and minimum intensities, and therefore it must be the case that $f(r) e^{*}(r)$ is real. If we also assume that the total incident intensities of the two fields are equal, $I_{e}=I_{f}=I$, then we can write the visibility of the interferometer in the simple form:

$$
V=\frac{\int f(r) e^{*}(r) d r}{I}
$$


This expression allows us to derive a number of relations

$$
\begin{aligned}
\iint|e(x) f(y)|^{2} d x d y= & \int|e(x)|^{2} d x \int|f(y)|^{2} d y=I^{2} \\
\iint e^{*}(x) f(x) e(y) f^{*}(y) d x d y=\int e^{*}(x) f(x) d x \int e(y) f^{*}(y) d y=I^{2} V^{2} & \\
\iint|e(x) f(y)-f(x) e(y)|^{2} d x d y= & \iint(e(x) f(y)-f(x) e(y)) \\
& \times\left(e^{*}(x) f^{*}(y)-f^{*}(x) e^{*}(y)\right) d x d y \\
= & \iint\left(|e(x)|^{2}|f(y)|^{2}+|f(x)|^{2}|e(y)|^{2}\right. \\
& -e^{*}(x) f(x) e(y) f^{*}(y) \\
& \left.-e(x) f^{*}(x) e^{*}(y) f(y)\right) d x d y \\
= & 2 I^{2}-2 I^{2} V^{2} \\
= & 2 I^{2}\left(1-V^{2}\right)
\end{aligned}
$$

that will be useful below.

Using the relations derived above, we can now write the spatial modes $\xi$ appearing in the density matrix of ion $b$ in terms of the measureable parameters $I$ and $V$. As above, we will assume the incident intensities are equal. We then get:

$$
\rho_{b}=\frac{I^{2}}{8}\left(\begin{array}{cc}
|\alpha|^{2}+|\beta|^{2}\left(1-V^{2}\right) & \alpha \beta^{*} V^{2} \\
\beta \alpha^{*} V^{2} & |\beta|^{2}+|\alpha|^{2}\left(1-V^{2}\right)
\end{array}\right)
$$

Of course, the factor of $I^{2} / 8$ in front should just be part of the normalization term we've been neglecting throughout this derivation. We will therefore discard it, and just use the condition $\operatorname{tr}\left(\rho_{b}\right)=1$ to determine the proper normalization. After doing so, we finally end up with the density matrix for ion $b$ as:

$$
\rho_{b}=\frac{1}{2-V^{2}}\left(\begin{array}{cc}
|\alpha|^{2}+|\beta|^{2}\left(1-V^{2}\right) & \alpha \beta^{*} V^{2} \\
\beta \alpha^{*} V^{2} & |\beta|^{2}+|\alpha|^{2}\left(1-V^{2}\right)
\end{array}\right)
$$


The above allows us to calculate the expected reduction in fidelity for any teleported state as a function of the visibility of the interferometer. For any given input state, we know the ideal density matrix is simply:

$$
\rho_{\text {ideal }}=\left(\begin{array}{cc}
|\alpha|^{2} & \alpha \beta^{*} \\
\beta \alpha^{*} & |\beta|^{2}
\end{array}\right)
$$

Earlier, we defined the fidelity as simply the overlap of the two states; this can be written succinctly as $\mathcal{F}=\operatorname{tr}\left(\rho_{b} \rho_{\text {ideal }}\right)$. Therefore, using the expressions above we find:

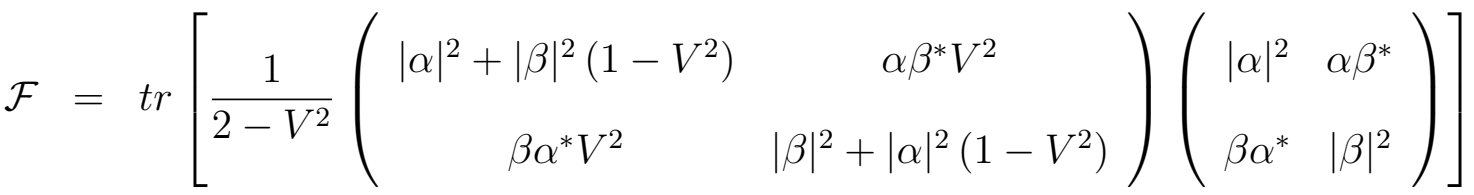

$$
\begin{aligned}
& =\frac{1}{2-V^{2}}\left(|\alpha|^{4}+|\beta|^{4}+2|\alpha|^{2}|\beta|^{2}\left(1-V^{2}\right)+2|\alpha|^{2}|\beta|^{2} V^{2}\right) \\
& =\frac{1}{2-V^{2}}\left(|\alpha|^{2}+|\beta|^{2}\right) \\
& =\frac{1}{2-V^{2}}
\end{aligned}
$$

We see that if the visibility of the interferometer is perfect $V=1$ then the fidelity is 1 ; whereas if the spatial mode-overlap is nonexistent, $V=0$, then the fidelity drops to $1 / 2$ (exactly what would be expected for a totally mixed state).

In the experiment, we measure the visibility of the interferometer by coupling laser light into the single-mode fibers used for transferring the spontaneously emitted photons from the atom to the beamsplitter. We find a visibility $V>0.98$. By the above derivation, we can thereby estimate that the spatial mode-mismatch at the beamsplitter reduces the fidelity of the teleportation protocol by at most about $4 \%$. 8.3.2 Imperfect state detection We calculate the expected degradation in fidelity due to imperfect state detection of the atomic qubit using the formalism of the measurement operators [31] used above. Let $\epsilon_{j}$ be the error in the measurement of 
the qubit state of ion $j=a, b$. The measurement operators may then be defined as:

$$
\begin{aligned}
& M_{0 j}=\sqrt{1-\epsilon_{j}}|0\rangle\left\langle 0\left|+\sqrt{\epsilon_{j}}\right| 1\right\rangle\langle 1| \\
& M_{1 j}=\sqrt{\epsilon_{j}}|0\rangle\left\langle 0\left|+\sqrt{1-\epsilon_{j}}\right| 1\right\rangle\langle 1|
\end{aligned}
$$

Note that these satisfy the completeness relation $I_{j}=\sum_{m} M_{m j}^{\dagger} M_{m j}$.

In this calculation we will assume the interference of the photons at the beamsplitter is perfect, so that the state of the two ions after a coincident detection of the photons and rotation of ion $a$ by $R_{y}(\pi / 2)$, we have the state (Eq. 8.4):

$$
\begin{aligned}
|\psi\rangle_{\text {ions }} & =\frac{1}{\sqrt{2}}|0\rangle_{a}\left(\alpha|1\rangle_{b}+\beta|0\rangle_{b}\right)+\frac{1}{\sqrt{2}}|1\rangle_{a}\left(\alpha|1\rangle_{b}-\beta|0\rangle_{b}\right) \\
& =\frac{1}{\sqrt{2}}|0\rangle(\alpha|1\rangle+\beta|0\rangle)+\frac{1}{\sqrt{2}}|1\rangle(\alpha|1\rangle-\beta|0\rangle)
\end{aligned}
$$

where in the second line we have again invoked the shorter notation of the prior section and suppressed the subscripts, instead designating the subsystems by the ordering of the states (the left-most always being $a$, the right-most $b$ ); if there is any chance of ambiguity, we will reinsert the subscripts at that point.

We now measure ion $a$ using our imperfect measurement operator. The following shows the explicit derivation for measuring the state $|0\rangle_{a}$; the derivation for the $|1\rangle_{a}$ is analogous, and after the conditional microwave rotation on ion $b$, yields exactly the same result. As in the previous section, the measurement completed by taking the partial trace over the measurement operator and the quantum state, so that the 
density matrix for $b$ is given by:

$$
\begin{aligned}
\rho_{b}= & \operatorname{tr}_{a}\left(M_{0 a}^{\dagger} M_{0 a}|\psi\rangle_{i o n s}\langle\psi|\right) \\
= & {\left[\sqrt{1-\epsilon_{a}}\left({ }_{a}\langle 0|\right)\left(\alpha|1\rangle_{b}+|0\rangle_{b}\right)+\sqrt{\epsilon_{a}}\left({ }_{a}\langle 1|\right)\left(\alpha|1\rangle_{b}-\beta|0\rangle_{b}\right)\right] } \\
& \otimes\left[\sqrt{1-\epsilon_{a}}|0\rangle_{a}\left(\alpha^{*}{ }_{b}\left\langle 1\left|+\beta^{*}{ }_{b}\langle 0|\right)+\sqrt{\epsilon_{a}} \mid 1\right\rangle_{a}\left(\alpha^{*}{ }_{b}\langle 1|-\beta^{*}{ }_{b}\langle 0|\right)\right]\right. \\
= & \left(1-\epsilon_{a}\right)\left(|\alpha|^{2}|1\rangle\left\langle 1\left|+\beta \alpha^{*}\right| 0\right\rangle\left\langle 1\left|+\alpha \beta^{*}\right| 1\right\rangle\left\langle\left. 0|+| \beta\right|^{2} \mid 0\right\rangle\langle 0|\right) \\
& +\epsilon_{a}\left(|\alpha|^{2}|1\rangle\left\langle 1\left|-\beta \alpha^{*}\right| 0\right\rangle\left\langle 1\left|-\alpha \beta^{*}\right| 1\right\rangle\left\langle\left. 0|+| \beta\right|^{2} \mid 0\right\rangle\langle 0|\right) \\
= & |\beta|^{2}|0\rangle\left\langle 0\left|+\left(1-2 \epsilon_{a}\right) \beta \alpha^{*}\right| 0\right\rangle\left\langle 1\left|+\left(1-2 \epsilon_{a}\right) \alpha \beta^{*}\right| 1\right\rangle\left\langle\left. 0|+| \alpha\right|^{2} \mid 1\right\rangle\langle 1|
\end{aligned}
$$

After measurement of ion $a$, a microwave pulse conditioned upon the measurement is applied to ion $b$. In the case of measuring $|0\rangle_{a}$, the rotation $R_{x}(\pi)$ is applied to ion $b$. Then density matrix is then:

$$
\rho_{b}=\left(\begin{array}{cc}
|\alpha|^{2} & \left(1-2 \epsilon_{a}\right) \alpha \beta^{*} \\
\left(1-2 \epsilon_{a}\right) \beta \alpha^{*} & |\beta|^{2}
\end{array}\right)
$$

Determining the effect on the fidelity in this case is not as straight-forward as in the previous section. Here the fidelity of the teleported state depends critically upon the state amplitudes. As can be seen in Eq. 8.39, if either $\alpha$ or $\beta$ is zero, then the imperfect detection on ion $a$ plays no role in the final density matrix of ion $b$. Intuitively, this is correct because the influence of imperfect measurement of ion $a$ is propagated by the conditional rotation on ion $b$. In the case that $\alpha$ or $\beta$ is zero, then the two possible rotations $R_{x}(\pi)$ and $R_{y}(\pi)$ perform the same action, and therefore the measurement on ion $a$ is inconsequential in this case.

The fidelity of the operation with imperfect measurements can be evaluated by reconstructing the density matrix following measurement of ion $b$ in the same fashion as is done with the actual experimental data. We define the probability of measuring a particular state $|\psi\rangle$ as $P_{|\psi\rangle}$. Since all the measurements occur via state dependent 
fluorescence, measurement in bases other than the $z$-basis are completed by performing a microwave rotation prior to detection. The six possible probabilities are then given by:

$$
\begin{aligned}
P_{|0\rangle} & =\operatorname{tr}\left(M_{0 b}^{\dagger} M_{0 b} \rho_{b}\right) \\
P_{|1\rangle} & =\operatorname{tr}\left(M_{1 b}^{\dagger} M_{1 b} \rho_{b}\right) \\
P_{|0\rangle-|1\rangle} & =\operatorname{tr}\left(M_{0 b}^{\dagger} M_{0 b}\left[R_{y}(\pi / 2) \rho_{b} R_{y}^{\dagger}(\pi / 2)\right]\right) \\
P_{|0\rangle+|1\rangle} & =\operatorname{tr}\left(M_{1 b}^{\dagger} M_{1 b}\left[R_{y}(\pi / 2) \rho_{b} R_{y}^{\dagger}(\pi / 2)\right]\right) \\
P_{|0\rangle-i|1\rangle} & =\operatorname{tr}\left(M_{1 b}^{\dagger} M_{1 b}\left[R_{x}(\pi / 2) \rho_{b} R_{x}^{\dagger}(\pi / 2)\right]\right) \\
P_{|0\rangle+i|1\rangle} & =\operatorname{tr}\left(M_{0 b}^{\dagger} M_{0 b}\left[R_{x}(\pi / 2) \rho_{b} R_{x}^{\dagger}(\pi / 2)\right]\right)
\end{aligned}
$$

These probabilities allow us to calculate the Stokes parameters:

$$
\begin{aligned}
& S_{0}=P_{|1\rangle}+P_{|0\rangle}=1 \\
& S_{1}=P_{|0\rangle+|1\rangle}-P_{|0\rangle-|1\rangle} \\
& S_{2}=P_{|0\rangle+i|1\rangle}-P_{|0\rangle-i|1\rangle} \\
& S_{3}=P_{|0\rangle}-P_{|1\rangle}
\end{aligned}
$$

These parameters are the coefficients of the Pauli matrices in a simple analytical formula for the reconstruction of the density matrix [159]:

$$
\rho_{b, \text { recon }}=\frac{1}{2} \sum_{j=0}^{3} S_{j} \hat{\sigma}_{j}
$$

The reconstructed density matrix contains the effect of imperfect measurement on ion $b$. We can then calculate the fidelity of the teleported state, taking into account imperfect detection, by $\mathcal{F}=\operatorname{tr}\left(\rho_{b, \text { recon }} \rho_{\text {ideal }}\right)$. Taking the state detection fidelities $\epsilon_{a}=0.985$ and $\epsilon_{b}=0.975$, we calculate the fidelity of the states $\{|0\rangle,|1\rangle,(|0\rangle+$ $|1\rangle) / \sqrt{2},(|0\rangle-|1\rangle) / \sqrt{2},(|0\rangle+i|1\rangle) / \sqrt{2},(|0\rangle-i|1\rangle) / \sqrt{2}\}$ to be $\{0.975,0.975,0.961$, $0.961,0.961,0.961\}$, yielding an average reduction in fidelity of about $3.5 \%$. 
Given that the fidelity of teleporting the two states $|0\rangle$ and $|1\rangle$ depend only on the measurement imperfection on ion $b$, it would have been beneficial to have reversed the roles of the two ions in this experiment. Doing so could have improved the average fidelity by $0.003 \%$. However, this change is within the error of the results presented here; the improvement would only be noticed in a much larger sample of events.

\subsection{Discussion}

As in the original teleportation proposal reviewed in Chap. II, the successful implementation of our teleportation protocol requires the transmission of two classical bits of information [7]: one to announce the success of the heralded quantum gate and another to determine the proper final rotation to recover the teleported state at ion $b$. While these classical bits do not convey any information about the quantum states of either ion $a$ or $b$, in the absence of this classical information ion $b$ is left in a mixed state (Eq. 8.5), and the protocol fails. The required classical communication ensures that no information is transferred faster than the speed of light.

However, the heralded teleportation protocol demonstrated here differs from the original proposal in several respects. In this case, we use four qubits (two atoms and two photons) rather than three, and our implementation is intrinsically probabilistic because the two-photon Bell states are not all deterministically distinguishable [148, 144, 64]. Nevertheless, the teleportation protocol succeeds without postselection, due to the two-photon coincident detection that serves as a heralding event [158]. The protocol presented here has the additional advantage of establishing the quantum channel between the (atomic) quantum memories using photons and entanglement swapping, allowing the atoms to be separated by a large distance from the outset. 
Ultimately, the teleportation scheme demonstrated here has the potential to form the elementary constituent of a quantum repeater capable of networking quantum memories over vast distances, and may be an essential protocol for the realization of scalable quantum computation. 


\section{CHAPTER IX}

\section{Conclusions and Outlook}

"What smells like blue?"

-Philip J. Fry, Futurama

The high fidelities obtained in the teleportation and quantum gate experiments is evidence of the excellent coherence properties of the photonic frequency qubit and the "clock" state atomic qubit. Together, these complimentary qubits may provide a robust system for applications in quantum information. In this final chapter, we explore the possible methods for scaling this system for practical quantum communication and quantum computation.

\subsection{Quantum gate with infrared photons}

In principle, any arbitrary distance can be bridged using a quantum repeater based on the entanglement protocols reviewed in prior chapters. However, the number of nodes needed to efficiently implement the quantum repeater is approximately proportional to the inverse of the attenuation length of the photons [28]. In order to establish quantum channels across long-distances, it may be more practical to use photons with wavelength in the infrared region of the spectrum (rather than the $369.5 \mathrm{~nm}$ photons used here), as these photons experience less attenuation in fiber. Moreover, access to additional optical frequencies may facilitate entanglement between disparate optically active systems, such as atoms and quantum dots. 
The rich atomic structure of the $\mathrm{Yb}^{+}$atom results in transitions across the optical spectrum. Two additional transitions that appear particularly amenable to the photon-mediated heralded gate described in Chapter VII are the $935 \mathrm{~nm}^{3}[3 / 2]_{1 / 2} \leftrightarrow$ ${ }^{2} D_{3 / 2}$ transition, and the $1.3 \mu \mathrm{m}{ }^{2} P_{3 / 2} \leftrightarrow{ }^{2} D_{3 / 2}$ transition.

Spontaneously emitted photons at $935 \mathrm{~nm}$ that are entangled with the internal electronic states of the atom can be generated as outlined below and illustrated in Fig. 9.1(a). For this protocol, we will initialize the atom in the ${ }^{2} S_{1 / 2}$ ground state, and excite the atom using ultrafast pulses at $297.1 \mathrm{~nm}$. Pulses at $297.1 \mathrm{~nm}$ could be generated by the third-harmonic generation of a mode-locked Ti:S laser operating at $891.4 \mathrm{~nm}$. Detection of the atomic state could still take place at $369.5 \mathrm{~nm}$. As long as the two atomic states are mapped to separate hyperfine manifolds in the ${ }^{2} D_{3 / 2}$ level, then selection rules can be exploited to transfer only population in the ${ }^{2} D_{3 / 2}|F=1\rangle$ manifold to ${ }^{2} S_{1 / 2}|F=1\rangle$ for state dependent fluorescence detection of the atom. Given the $52 \mathrm{~ms}$ natural lifetime of the ${ }^{2} D_{3 / 2}$ level, it should be possible to obtain state detection fidelities $>98 \%[61]$.

A heralded quantum gate can be performed using $935 \mathrm{~nm}$ photons (Fig. 9.1(a)) by first preparing the atom in a superposition of $|0\rangle$ and $|1\rangle$ :

$$
|\psi\rangle_{a}=\alpha|0\rangle+\beta|1\rangle
$$

A $\pi$-polarized ultrafast pulse at $297.1 \mathrm{~nm}$ is used to coherently transfer the population from ${ }^{2} S_{1 / 2}$ to ${ }^{3}[3 / 2]_{1 / 2}$. Due to the selection rules involved, this will transfer $|0\rangle={ }^{2} S_{1 / 2}\left|F=0, m_{F}=0\right\rangle$ to ${ }^{3}[3 / 2]_{1 / 2}\left|F=1, m_{F}=0\right\rangle$ and $|1\rangle={ }^{2} S_{1 / 2} \mid F=$ $\left.1, m_{F}=0\right\rangle$ to ${ }^{3}[3 / 2]_{1 / 2}\left|F=0, m_{F}=0\right\rangle$. The ${ }^{3}[3 / 2]_{1 / 2}$ level can then decay to ${ }^{2} D_{3 / 2}$ by spontaneously emitting a $935 \mathrm{~nm}$ photon. By collecting photons at 935 nm emitted perpendicular to the quantization axis, we can use polarization filters to distinguish $\pi$ - and $\sigma$-polarized photons. A $\pi$-polarized $935 \mathrm{~nm}$ photon is the result 


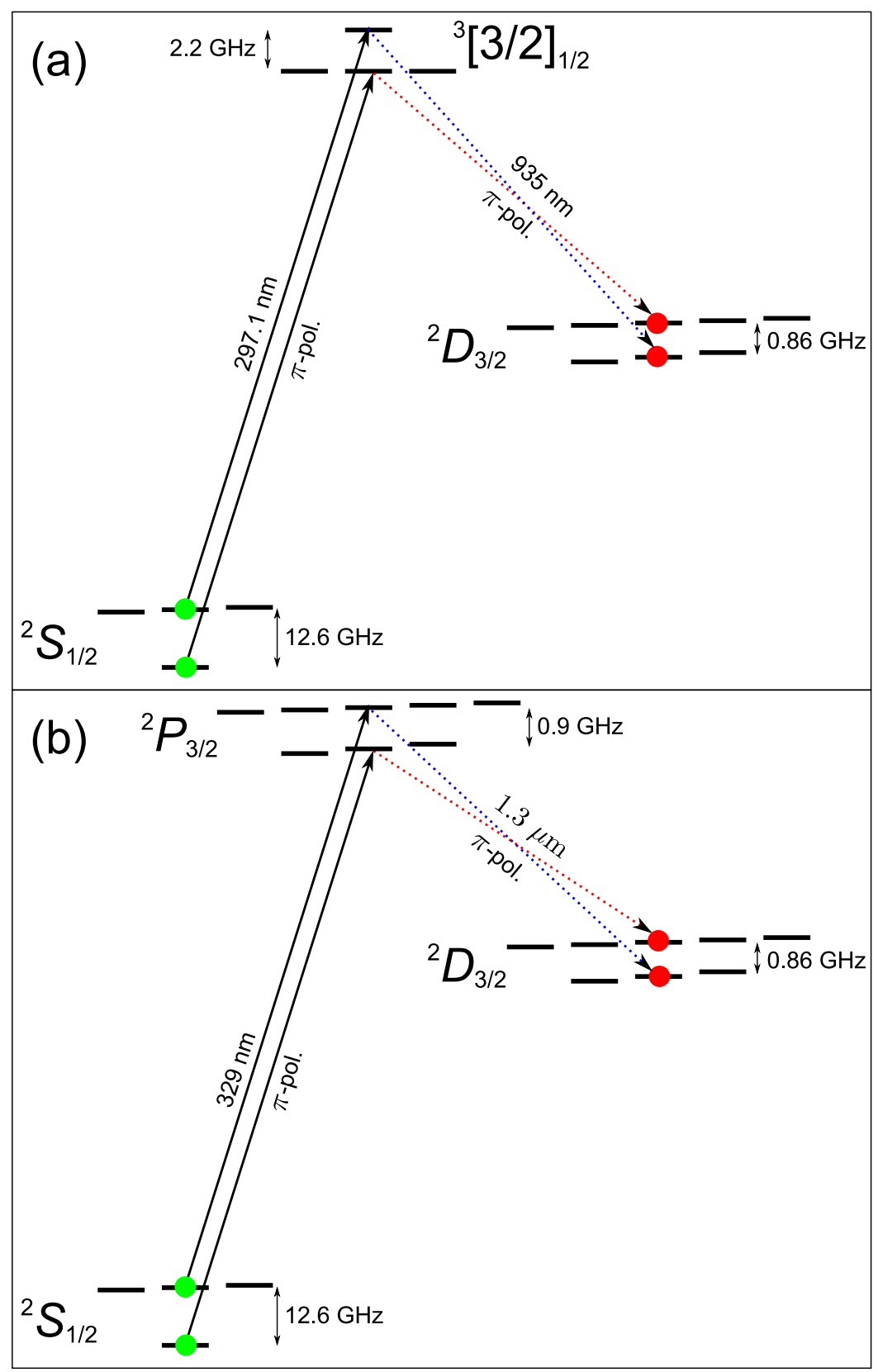

Figure 9.1: Quantum gate protocols utilizing infrared transitions ${ }^{171} \mathrm{Yb}^{+}$. (a) Procedure for generating photons at $935 \mathrm{~nm}$ with frequency mode entangled with the ${ }^{2} D_{3 / 2} \mid F=1,2, m_{F}=$ $0\rangle$ atomic state. As this protocol retains initial coherence in the atom, it is suitable for the implementation of a heralded quantum gate. (b) Method of generating $1.3 \mu \mathrm{m}$ photons for the implementation of a heralded quantum gate. 
of a ${ }^{3}[3 / 2]_{1 / 2}\left|F=1, m_{F}=0\right\rangle$ to ${ }^{2} D_{3 / 2}\left|F=2, m_{F}=0\right\rangle$ or ${ }^{3}[3 / 2]_{1 / 2}\left|F=0, m_{F}=0\right\rangle$ to ${ }^{2} D_{3 / 2}\left|F=1, m_{F}=0\right\rangle$ transition, resulting in the frequency of the emitted photon being entangled with the internal state of the atom:

$$
|\psi\rangle_{a p}=\beta\left|F=2, m_{F}=0\right\rangle\left|\nu_{r}\right\rangle+\alpha\left|F=1, m_{F}=0\right\rangle\left|\nu_{b}\right\rangle
$$

where here $\left|F, m_{F}\right\rangle$ refer to states in the ${ }^{2} D_{3 / 2}$ level, and $\Delta \nu=\nu_{b}-\nu_{r}=3.07 \mathrm{GHz}$ is the sum of the ${ }^{2} D_{3 / 2}$ and ${ }^{3}[3 / 2]_{1 / 2}$ hyperfine splittings (Chap. IV). As the ionphoton entanglement process preserved the coherence initially present in the ion, the interference and detection of these photons can be used to implement a heralded quantum gate $[66,143]$. Detection of the atomic state can be accomplished by transferring the ${ }^{2} D_{3 / 2}\left|F=1, m_{F}=0\right\rangle$ to the ${ }^{2} S_{1 / 2}|F=1\rangle$ for fluorescence detection at $369.5 \mathrm{~nm}$. Light from a $935 \mathrm{~nm} \mathrm{cw}$ laser could be used drive the ${ }^{2} D_{3 / 2} \mid F=$ $1\rangle \leftrightarrow{ }^{3}[3 / 2]_{1 / 2}|F=0\rangle$ transition to transfer population in the ${ }^{2} D_{3 / 2}|F=1\rangle$ manifold to ${ }^{2} S_{1 / 2}|F=1\rangle$. The state of the atom is then determined by resonantly driving the ${ }^{2} S_{1 / 2}|F=1\rangle \leftrightarrow{ }^{2} P_{1 / 2}|F=0\rangle$ transition, and detecting photons at $369.5 \mathrm{~nm}$; detecting fluorescence at $369.5 \mathrm{~nm}$ indicates the atom was originally in the $\mid F=$ $\left.1, m_{F}=0\right\rangle$ state, whereas the absence of fluorescence indicates the atom is in $\mid F=$ $\left.2, m_{F}=0\right\rangle$.

The disadvantage of generating $935 \mathrm{~nm}$ photons is that the branching ratio of the ${ }^{3}[3 / 2]_{1 / 2}$ level between ${ }^{2} S_{1 / 2}$ and ${ }^{2} D_{3 / 2}$ has been calculated to be about 55:1 [89], decreasing the probability of generating a $935 \mathrm{~nm}$ photon and thereby reducing the overall success probability of the entanglment and gate protocols outlined above. Due to this reduced success probability, dark counts of the single-photon detectors could become a significant source of error. However, there is a way to "veto" the contribution of dark counts in this protocol. After a photon detection event, a microwave pulse at the ${ }^{2} D_{3 / 2}$ hyperfine splitting of $0.86 \mathrm{GHz}$ can be used to transfer 
the ${ }^{2} D_{3 / 2}\left|F=1, m_{F}=0\right\rangle$ atomic state to, for instance, the ${ }^{2} D_{3 / 2}\left|F=2, m_{F}=1\right\rangle$ state. If the fluorscence detection procedure outlined previously is now performed, no $369.5 \mathrm{~nm}$ photons should be detected. ${ }^{1}$ The population transfered from ${ }^{2} D_{3 / 2}|F=1\rangle$ to ${ }^{2} D_{3 / 2}|F=2\rangle$ can be returned by a second microwave pulse, and the remainder of the protocol completed. On the other hand, detection of $369.5 \mathrm{~nm}$ photons during this detection interval would indicate that the $935 \mathrm{~nm}$ "detection" was a false event, and should be discarded.

The gate protocol outlined above could also be implemented at $1.3 \mu \mathrm{m}$, where attenuation in optical fiber is near a minimum (Fig. 9.1(b)). In this case, the atom is initially prepared ${ }^{2} S_{1 / 2}$, and then excited to ${ }^{2} P_{3 / 2}$ by an ultrafast pulse at $329 \mathrm{~nm}$. Decay from ${ }^{2} P_{3 / 2}$ to ${ }^{2} D_{3 / 2}$ results in the emission of a $1.3 \mu \mathrm{m}$ photon. While the above protocol can be implemented in an analogous fashion at this wavelength, the branching ratio from ${ }^{2} P_{3 / 2}$ to ${ }^{2} S_{1 / 2}$ versus ${ }^{2} D_{3 / 2}$ is about 475:1 [89]. Thus, while the wavelength is more amenable to long-distance transmission, the decrease in the protocol success probability is even more dramatic. In addition, ${ }^{2} P_{3 / 2}$ can also decay to ${ }^{2} D_{5 / 2}$, which can subsequently decay to the long-lived ${ }^{2} F_{7 / 2}$. Depopulating these additional metastable states would require additional optical frequencies and/or limit the repetition rate of the experiment.

\subsection{Scalability}

The primary impediment to scaling the current setup to more qubits is the success probability of the heralded quantum gate. As discussed in Chap. VII, there are several avenues that can be pursued to improve the success rate. Given that the effective solid angle of collection is about an order of magnitude smaller than the

\footnotetext{
${ }^{1}$ Here, the $14.7 \mathrm{GHz}$ sideband usually used during Doppler cooling can be applied to the impinging $369.5 \mathrm{~nm}$ light during this detection interval, to ensure population left in the $|0\rangle$ state also results in emitted 369.5 nm photons.
} 
other contributing factors in Eq. 7.10, improvements here are bound to have the greatest impact.

The effective solid angle of collection can be increased through several mechanisms. The first proposal is to situate reflective or refractive optics near the trapped atomic ion. Indeed, this route is already being pursued both within our research group and elsewhere $[162,163]$. Perhaps one of the most intriguing approaches would be to place the trapped ion at the focal point of a parabolic mirror, allowing the collection efficiency to approach unity $[164,165]$. If properly segmented, the mirror (assumed metallic for this scenario) could also serve as the electrodes for the ion trap. Alternatively, the spontaneous emission into free space could be replaced by the induced emission into the small mode volume of a high finesse cavity, which can reach near unit efficiency $[166,167]$. Even though the free spectral range of the cavity would have to be $14.7 \mathrm{GHz}$ to simultaneously support both frequency modes, choosing a near-concentric design could still result in a small mode volume and thus in a high emission probability into a well-defined Gaussian mode. In any case, the placement of optics near the ion will require careful assessment to ensure the added structures do not destabilize the quadrupole trap.

While improvements that increase the success probability of the gate operation can enhance scalability, even with a low success probability, this gate can still be efficiently scaled to more complex systems. In a quantum repeater architecture that allows for local deterministic gates to be performed at each node, the photonmediated operations between nodes can be attempted simultaneously. The requirement for scaling to more nodes is therefore only that the coherence time of the atoms exceed the time needed to connect all the nodes of the quantum repeater. Given all connections can be attempted simultaneously, then the time needed to connect all 
nodes is approximately given by $T_{\text {success }} \ln (N-1)$, where $T_{\text {success }}$ is the average time needed to connect two nodes and $N$ is the total number of nodes in the repeater $(N \geq 2)$. With a measured coherence time of 2.5 seconds for the ${ }^{171} \mathrm{Yb}^{+}$hyperfine qubit (Chap. IV), this means that to establish entanglement over a quantum repeater with 10 nodes, we require the average success rate to be greater than about $1 \mathrm{~Hz} .^{2}$ With modest improvements in the photon collection efficiency and repetition rate of the experiment (limited by the microwave $\pi$ time) it should be possible to achieve this success rate.

The stipulations on the success probability for generating large cluster states for scalable quantum computing are more stringent. The time needed to construct an $n$ qubit $1 \mathrm{D}$ cluster state is approximately given by [142]:

$$
T(n) \approx t_{a}\left(\frac{1}{P_{\text {success }}}\right)^{\log _{2}\left(n_{c}+1\right)}+\left(\frac{t_{a}}{P_{\text {success }}}\right) \log _{2}\left(n-n_{c}\right)
$$

where $n_{c} \approx 4 / P_{\text {success }}$ is the critical number of qubits in a single $1 \mathrm{D}$ cluster state chain that must be generated before multiple chains can be fused together. Clearly, even if we assume the repetition rate of the experiment can be improved to $100 \mathrm{~ns}$, at our current success probability of $2.2 \times 10^{-8}$ the time to generate a 100 qubit cluster state is prohibitively long ( $>10^{36}$ years). However, if the improvements suggested above are able to increase the gate success probability to $10 \%$, then a 100 qubit cluster state could be generated in less than $25 \mathrm{~ms}$.

\subsection{Summary}

We have demonstrated teleportation of quantum information between quantum memories over a distance of about one meter. The protocol relied upon the implementation of heralded quantum gate based on the interference and detection of

\footnotetext{
${ }^{2}$ In this estimate, we have assumed only one photon-mediated connection is being attempted between each node. Multiplexing this operation further relaxes the required success rate.
} 
spontaneously emitted photons. The high fidelity attained in these experiments demonstrates the applicability of the photonic frequency qubit and the $\mathrm{Yb}^{+}$atomic qubit to quantum information science. Ultimately, the quantum gate and teleportation protocol demonstrated here could be essential to realizing long-distance quantum communication and scalable quantum computation. 
APPENDICES 


\section{APPENDIX A}

\section{RSA Algorithm}

\section{A.1 Number Theory Basics}

One of the motivations for quantum computation is the unprecendented codebreaking it could enable. As mentioned earlier, this is because many encryption methods rely on the relative inability of a conventional computer to factor large numbers. However, it not intuitively obvious what factorization has to do with encryption. Therefore, in this appendix we outline the methodology employed by the RSA algorithm to send encrypted messages via prime factorization.

Most of the material covered here can be found in [168], as well as many other introductory texts on number theory. Since number theory deals with the set of integers, all numbers referred to in this appendix should be assumed to be an integer, unless otherwise stated. We now start with some definitions.

Definition Given two integers, $a$ and $b, a$ is said to be a divisor of $b$ (or $a$ divides $b$ ) if there exists some integer $x$ such that $b=a x$; we then write $a \mid b$. If there is no such $x$, then $a$ does not divide $b$, and we write $a \nmid b$.

Clearly, any number greater than $a$ (or smaller than $-a$ ) will not divide $a$. Since there are a finite number of divisors of $a$, there is a maximum or greatest divisor of $a$. 
Definition An integer $a$ is defined to be a common divisor of $b$ and $c$ if $a \mid b$ and $a \mid c$. As there are only a finite number of common divisors for any given $b$ and $c$, there is a greatest common divisor (gcd), denoted by $(b, c)$.

We are all familiar with the concept of a prime number: a number divisible only by itself and 1 (among the positive integers). Put another way, a prime number $p$ is a number such that given any other integer $a$, if $p \nmid a$, then $(p, a)=1$. The generalization of this idea is our next definition.

Definition If $(a, b)=1$, then we say $a$ and $b$ are relatively prime (also called coprime).

As it turns out, the concept of prime and relatively prime is vital to a large portion of number theory. There is even a function defined to say how many numbers are relatively prime to a given number.

Definition The number $\phi(m)$, known as Euler's $\phi$-function or the totient, is the number of positive integers less than or equal to $m$ that are relatively prime to $m$.

Definition Given an integers $a, b$, and $m(m \neq 0)$, we say $a$ is congruent to $b$ modulo $m$ if $m \mid(a-b)$, and write $a \equiv b(\bmod m)$.

Perhaps the easiest way to explain the idea of a modulus is via analogy with a clock. Basically, every hour of the day is congruent to one of the numbers in the set $\{1,2,3,4,5,6,7,8,9,10,11,12\} \bmod 12$. Comparing 24 and 12 hour clocks, we all know 13 is the same as $1 \mathrm{pm}$. Alternatively, we could say $13 \equiv 1(\bmod 12)$. Generally, working modulo some number simplifies things (after all, you're then working with a finite set of numbers, instead of the infinite set of integers) and can be used the exhibit some general properties of a particular set or class of numbers. An important result is stated (without proof) below. 
Theorem A.1.1 (Euler's Generalization of Fermat's Little Theorem) Given two integers $a$ and $m$, if $(a, m)=1$ then

$$
a^{\phi(m)} \equiv 1(\bmod m)
$$

The above theorem is an essential result of number theory, and will be used in the proof of the key lemma used in the RSA algorithm (RSA comes from Rivest, Shamir, and Adleman, the last names of the MIT inventors).

\section{A.2 Mathematics of Public-Key Cryptography}

Given the definitions and proofs above, we can now discuss how to transmitted an encrypted message over a public communication channel, where the encoding is accomplished through prime factorization. The method results from the following lemma.

Lemma A.2.1 Suppose $m$ is a positive integer and $(a, m)=1$. If $k$ and $\bar{k}$ are positive integers such that $k \bar{k} \equiv 1(\bmod \phi(m))$, then $a^{k \bar{k}} \equiv a(\bmod m)$.

Proof By the definition of congruence, we know there exists some positive integer $r$ such that $k \bar{k}=1+r \phi(m)$. Then, using Thm. A.1.1, we find

$$
a^{k \bar{k}}=a \cdot a^{r \phi(m)}=a\left(a^{\phi(m)}\right)^{r} \equiv a \cdot 1^{r} \equiv a(\bmod m)
$$

And this completes the proof.

How do you use the lemma above to transmit encrypted data? Well, consider the following the scenario. Suppose you want to send a message to the Secret Intelligence Service (SIS). The SIS is, of course, receiving secret message all the time. To make the transfer of information simple for their agents, they have made two numbers, $k$ and $m$ publicly available. What numbers did they choose? Well, basically, they just 
picked two really big prime numbers, $p_{1}$ and $p_{2}$ (say, 100 digits each), and multiplied them together to get $p_{1} p_{2}=m$. Since they know the prime factorization of $m$, they also know what $\phi(m)$ is, and choose some number $k$ such that $k<\phi(m)$ and $(k, \phi(m))=1$. Again, after doing all of this, they make $k$ and $m$ publicly available.

Knowing the SIS's method, and being well-versed in number theory yourself, you would first convert your message to numeric form (say, using the three digit American Standard Code for Information Interchange: ASCII). Let's call the numeric form of your message $a$. You then look up the current $k$ and $m$ being used by the SIS, and quickly calculate the unique number $b$, where $0 \leq b<m$, such that $b \equiv a^{k}(\bmod m)$. You then send the number $b$ to the SIS. You could even use a public channel; for example, you may just post it on the internet. Upon receiving the number $b$ from you, the SIS simply uses Lemma A.2.1 to decode the message. Since the SIS knows $\phi(m)$, they quickly calculate $\bar{k}$, and then employ Lemma A.2.1 to find $b^{\bar{k}} \equiv a(\bmod m)$.

In principle, of course, some random guy may come across $b, k$, and $m$, and may try to figure out the original message $a$. All he needs to do is factor $m$, and that would yield $\phi(m)$ and therefore $\bar{k}$. In practice, though, the task of factoring a 200 digit number, even using the best algorithms and the fastest classical computer available, would take prohibitively long.

\section{A.3 RSA Today}

A leader in encryption today is RSA laboratories. In an effort to maintain the highest level in encryption, RSA labs used to post 'challege numbers' on their website and offered cash prizes to the first person or team to factor them correctly. The largest number factored before the challenge was closed was RSA-200, which had 
200 decimel digits:

$$
\begin{aligned}
& \text { RSA- } 200= \\
& 27997833911221327870829467638722601621070446786955 \\
& 42853756000992932612840010760934567105295536085606 \\
& 18223519109513657886371059544820065767750985805576 \\
& 13579098734950144178863178946295187237869221823983
\end{aligned}
$$

The factors were found to be:

$$
\begin{aligned}
& 35324619344027701212726049781984643686711974001976 \\
& 25023649303468776121253679423200058547956528088349
\end{aligned}
$$

and

$$
\begin{aligned}
& 79258699544783330333470858414800596877379758573642 \\
& 19960734330341455767872818152135381409304740185467
\end{aligned}
$$

The team that factored it used a cluster of $802.2 \mathrm{GHz}$ CPUs and the general number field sieve (GNFS) algorithm. The factorization began in late 2003 and was completed in May 2005, with an equivalent time of about 55 years on a single $2.2 \mathrm{GHz}$ CPU. 


\section{APPENDIX B}

\section{Simulation Analysis in Mathematica}

In section 3.2.2, we outlined how to perform the analysis of the electrostatic simulation data obtained from CPO to model the properties of the ion trap. This appendix presents an example of the code used to perform this analysis in Mathematica.

First, the simulation data generated by CPO is imported:

ClearAll [RFTable, RFfunc, DCTable, DCfunc] ;

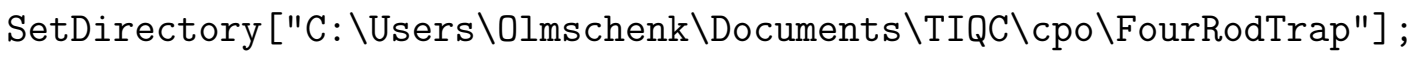

RFTable:=Import ["rf_rods_xz.txt", "Table"];

RFfunc=Interpolation [RFTable];

DCTable:=Import ["dc_needles_xy.txt", "Table"];

DCfunc=Interpolation [DCTable] ;

Note that for this example, we've only taken two planes of the simulation. Additional slices/planes would be required to model the full 3D potential.

Next we define a few parameters for use in the analysis code.

Clear [qe, Omegat, mion, RFvolt, dcorrection, freq, RFEmag2, DCvolt] ;

$\mathrm{qe}=1.602 / 10^{\wedge} 19$ 
Omegat=2 pi $3810^{\wedge} 6$;

$\operatorname{mion}=(171$ 1.66)/10^27;

RFvolt=1000;

DCvolt $=80$;

dcorrection=1/10^3;

freq[a2_] :=Sqrt [(2 a2 qe)/(dcorrection`2 mion)]/(2 pi);

where the term "dcorrection" is used to account for the fact that the simulation was performed in $\mathrm{mm}$, and here we use standard SI units for all other parameters. The function "freq" will be used to calculate the resulting secular frequency from the coefficient of a the quadratic term in a fit to the pseudopotential.

We now create the pseudopotential.

$\operatorname{RFEmag} 2\left[\mathrm{x}_{-}, z_{-}\right]=(\operatorname{Abs}[D[\operatorname{RFfunc}[\mathrm{x}, 0, \mathrm{z}], \mathrm{x}]$

Conjugate $[D[\operatorname{RFfunc}[\mathrm{x}, 0, \mathrm{z}], \mathrm{x}]]]+\operatorname{Abs}[\mathrm{D}[\operatorname{RFfunc}[\mathrm{x}, 0, \mathrm{z}], \mathrm{z}]$

Conjugate $[D[\operatorname{RFfunc}[\mathrm{x}, 0, \mathrm{z}], \mathrm{z}]]]$ ) /Evaluate [dcorrection $]^{`} 2$;

Clear [Poten] ;

Poten $\left[\mathrm{x}_{-}, \mathrm{y}_{-}, \mathrm{z}_{-}\right]:=\left(\left(\mathrm{RFvolt}^{\wedge} 2 \mathrm{qe} \mathrm{e}^{\wedge}\right) \operatorname{RFEmag} 2[\mathrm{x}, \mathrm{z}]\right) /(4$ mion Omegat`2 $)$ $+($ qe DCvolt) DCfunc $[x, y, 0]$;

As discussed in Chap. III, the Hessian matrix can be used to determine the principle axes.

AllBlue $\left[s_{-}\right]:=$Hue $[0.5+0.4 \mathrm{~s}]$;

Clear [vHessn, Hessn, eigenH, Theta1, Theta2, principle1, principle2, plotpotenNN, myrange] ;

vHessn $=D[$ Poten $[x, 0, z],\{\{x, z\}, 2\}]$;

Hessn $=$ vHessn $/ .\left\{x_{-}>0, z^{->0}\right\}$ 
eigenH $=$ Eigenvectors $[$ Hessn $]$
Theta1 $=\operatorname{ArcTan}[\operatorname{eigenH}[[1,1]] /$ eigenH $[[1,2]]] ;$
Theta2 $=\operatorname{ArcTan}[\operatorname{eigenH}[[2,1]] /$ eigenH $[[2,2]]] ;$

Finally, we do a quadratic fit along the principle axes, and use this fit to determine the expected secular frequencies.

Clear [P1axis, a2p1, P2axis, a2p2, myfitr] ;

myfitr=0.2;

myfits=0.01;

Print["The secular frequencies along the principle axes..."]

P1axis [w_] :=Fit $[$ Table $[\{\mathrm{v}, \operatorname{Poten}[\mathrm{v} \operatorname{Cos}[$ Theta1], 0,v Sin $[$ Theta1] $] /$ qe $\}$, $\{\mathrm{v},-$ myfitr, myfitr, myfits $\left.\}],\left\{1, \mathrm{w}, \mathrm{w}^{\wedge} 2\right\}, \mathrm{w}\right]$;

a2p1=1/2 D [(Evaluate $[\mathrm{P} 1 \mathrm{axis}[\mathrm{ww}]] /$. Ww $\rightarrow \mathrm{x}),\{\mathrm{x}, 2\}] / . \mathrm{x}->0$;

P1freq=freq $[\mathrm{a} 2 \mathrm{p} 1]$

P2axis [w_] :=Fit $[$ Table $[\{\mathrm{v}, \operatorname{Poten}[\mathrm{v}$ Cos [Theta2], 0,v Sin $[$ Theta2] $] / \mathrm{qe}\}$, $\{\mathrm{v},-$ myfitr, myfitr, myfits $\left.\}],\left\{1, \mathrm{w}, \mathrm{w}^{\wedge} 2\right\}, \mathrm{w}\right]$;

a2p2=1/2 D $[($ Evaluate $[\mathrm{P} 2 \operatorname{axis}[\mathrm{ww}]] /$. Ww $\rightarrow \mathrm{x}),\{\mathrm{x}, 2\}] / . \mathrm{x}->0$;

P2freq=freq [a2p2];

P3axis $\left[w_{-}\right]:=F i t[T a b l e ~[\{v, P o t e n[0, v, 0] / q e\}$, $\{\mathrm{v},-$ myfitr, myfitr, myfits $\left.\}],\left\{1, \mathrm{w}, \mathrm{w}^{\wedge} 2\right\}, \mathrm{w}\right]$;

a2p3=1/2 D $[($ Evaluate $[\mathrm{P} 3 a x i s[w w]]$. Ww $\rightarrow \mathrm{x}),\{\mathrm{x}, 2\}] / . \mathrm{x}->0$;

P3freq=freq [a2p3];

Print ["...are ",Style[P1freq,FontColor->Hue[0.]], ", ", Style[P2freq,FontColor->Hue[0.3] ], " and ", Style [P3freq,FontColor->Hue [0.6] ] , " Hz." ] ; 


\section{APPENDIX C}

\section{Reference Cavity}

A simple invar optical cavity is used for stabilization of the $739 \mathrm{~nm}$ and $935.2 \mathrm{~nm}$ lasers (Sec. 4.3). The cavity mirrors are housed in a machined invar 36 (iron-nickel

alloy containing 35-36\% nickel) rod, due to the extremely low thermal expansion of this metal [169].

A diagram of the final constructed cavity is shown in Fig. C.1, and a technical schematic is given in Fig. C.2. The first mirror is held in place by standard Thorlabs retaining rings. A tube piezo can be ramped by a high-voltage supply to scan the cavity and observe the transmission of incident light, or may be locked to a particular cavity length by the PID using the error signal from the iodine saturated absorption setup (Chap. IV). The second mirror is initially mounted external to the cavity, and aligned so that the cavity is confocal. It is then secured into place by a small amount of epoxy, and the external mount is removed. 


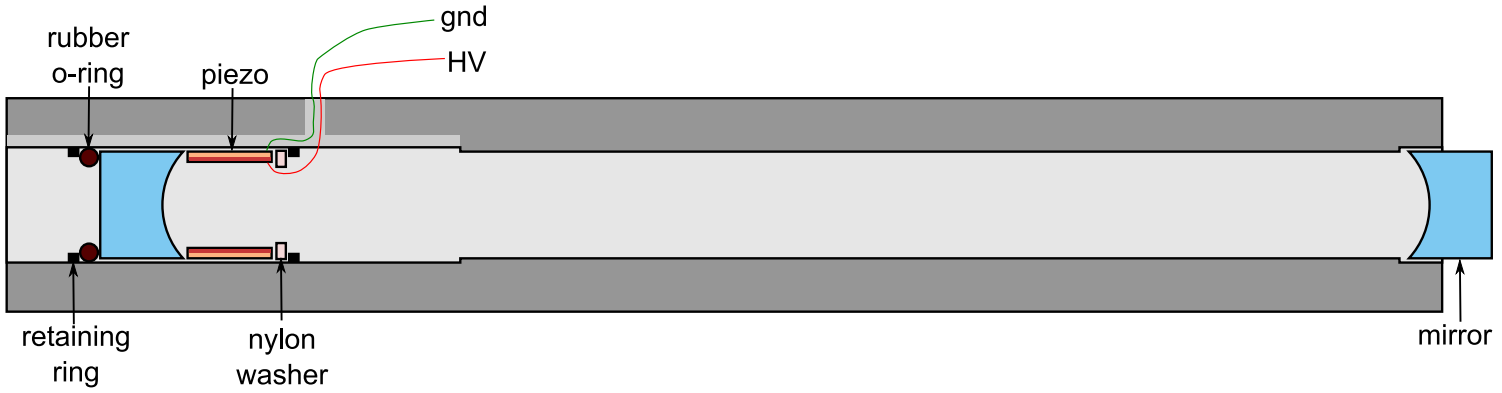

Figure C.1: Invar cavity assembly diagram. The components on the left (mirror, piezo tube, rubber o-ring and nylon washer, all held in place by two retaining rings) are assembled prior to alignment. The second mirror is initially mounted on an external optics mount that can be controllably translated along the axis of the cavity. As the cavity is scanned with light incident on the back face of either mirror, the secondary mirror is adjusted to make the cavity confocal. It is then secured with a dab of epoxy, and the external mount removed. 


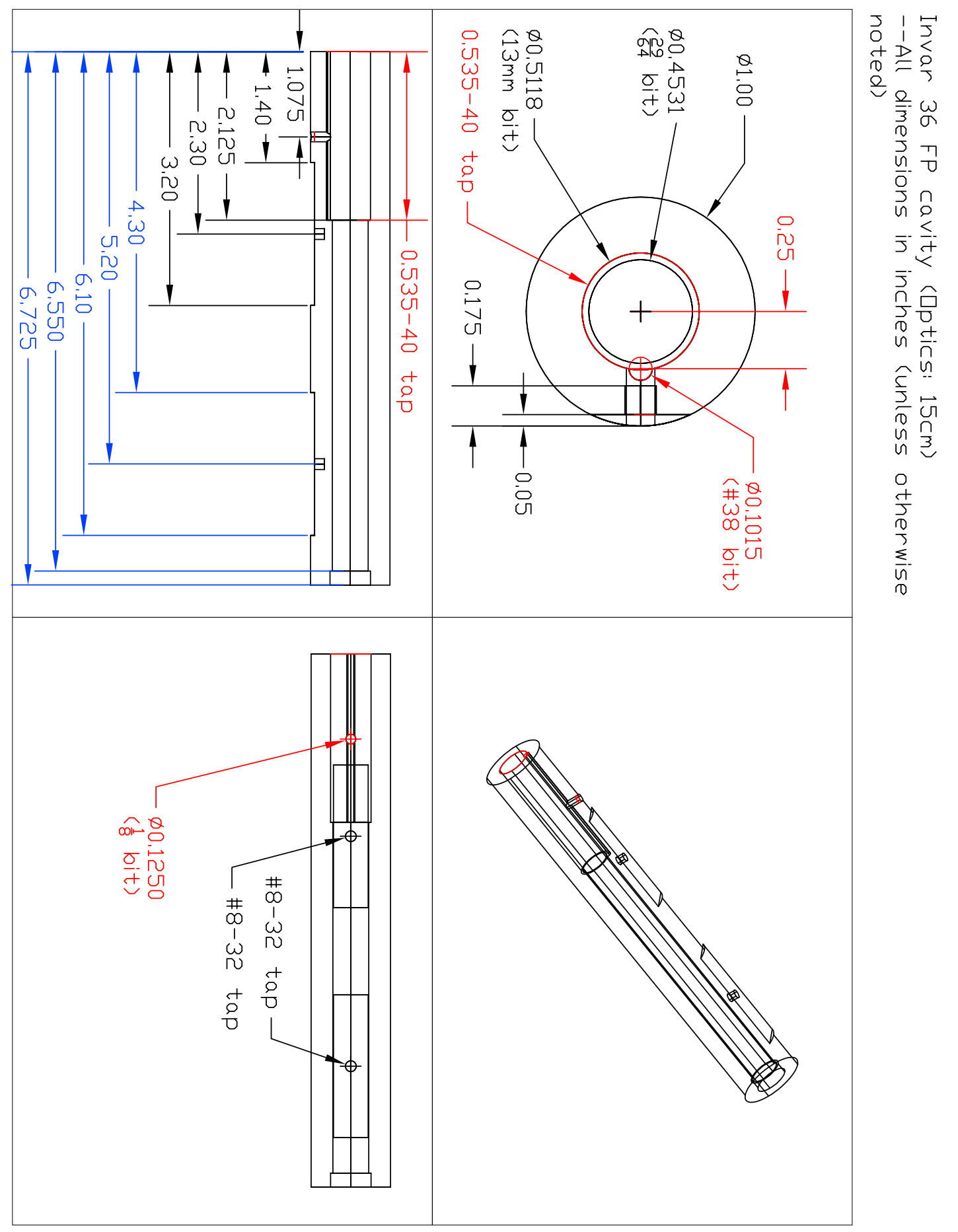

Figure C.2: Invar cavity dimensions. AutoCAD schematic of the invar cavity, with all dimensions in inches. Note the thread is a standard Thorlabs tap. 


\section{APPENDIX D}

\section{Radiation Polarization}

When the ion decays, it spontaneously emits a photon. If we define the quantization axis using an external magnetic field aligned along the $z$-axis, then the polarization and intensity of a given decay has an angular distribution governed by the type of multipole radiation associated with it. For a semi-classical, intuitive picture, see pages 244-245 of Ref. [170].

We define the angular momentum operator to be

$$
\mathbf{L}=-i(\mathbf{r} \times \nabla)
$$

Given this definition, note that $\mathbf{r} \cdot \mathbf{L}=0$ (see Ref. [111], pages 428-429 for additional formula). Using the angular momentum operator, we define the normalized, vector spherical harmonics to be

$$
\mathbf{X}_{\ell m}(\theta, \phi)=\frac{1}{\sqrt{\ell(\ell+1)}} \mathbf{L} Y_{\ell m}(\theta, \phi)
$$

Then, according to [111], page 437, the time-averaged power radiated per unit solid angle is

$$
\frac{d P}{d \Omega}=\frac{Z_{0}}{2 k^{2}}\left|\sum_{\ell, m}(-i)^{\ell+1}\left[a_{E}(\ell, m) \mathbf{X}_{\ell m} \times \mathbf{n}+a_{M}(\ell, m) \mathbf{X}_{\ell m}\right]\right|^{2}
$$

Since we are interested in the electric multipole fields, the angular distribution is

$$
\frac{d P}{d \Omega} \propto\left|\mathbf{X}_{\ell m} \times \mathbf{n}\right|^{2}
$$




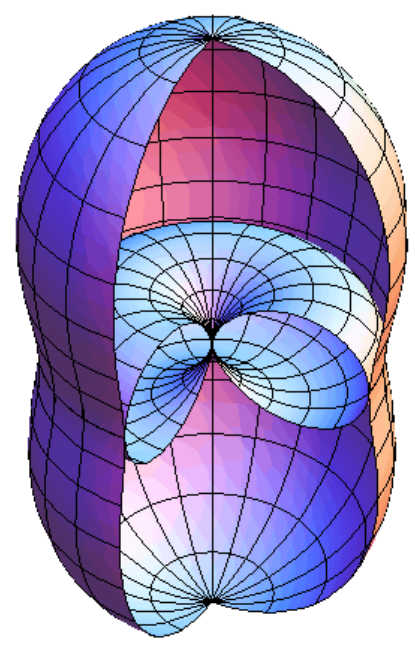

Figure D.1: The radiation patterns of $\left|\mathbf{X}_{10} \times \mathbf{r}\right|^{2}$ (inner, "donut" shape) and $\left|\mathbf{X}_{1-1} \times \mathbf{r}\right|^{2}+$ $\left|\mathbf{X}_{11} \times \mathbf{r}\right|^{2}$ (outer, "peanut" shape). Notice that $\left|\mathbf{X}_{1-1} \times \mathbf{r}\right|^{2}+\left|\mathbf{X}_{10} \times \mathbf{r}\right|^{2}+\left|\mathbf{X}_{11} \times \mathbf{r}\right|^{2}=$ 1 for all angles, as one would expect.

for a pure multipole.

\section{D.1 Radiation from $\Delta \ell=1, \Delta m=0$ transitions}

In spherical coordinates, the propagation of the radiation is along $\hat{\mathbf{r}}$, so $\mathbf{n}=\hat{\mathbf{r}}$. Then, for $\ell=1, m=0$, we find

$$
\begin{aligned}
\mathbf{X}_{10}(\theta, \phi) \times \mathbf{r} & =\frac{1}{\sqrt{2}}\left[-i(\mathbf{r} \times \nabla) Y_{10}(\theta, \phi)\right] \times \hat{\mathbf{r}} \\
& =\frac{-i}{\sqrt{2}}\left[(\mathbf{r} \times \nabla) \sqrt{\frac{3}{4 \pi}} \cos \theta\right] \times \hat{\mathbf{r}} \\
& =-i \sqrt{\frac{3}{8 \pi}}\left(r \hat{\mathbf{r}} \times\left[\hat{\mathbf{r}} \frac{\partial}{\partial r}+\hat{\theta} \frac{1}{r} \frac{\partial}{\partial \theta}+\hat{\phi} \frac{1}{r \sin \theta} \frac{\partial}{\partial \phi}\right] \cos \theta\right) \times \hat{\mathbf{r}} \\
& =-i \sqrt{\frac{3}{8 \pi}}\left[\left(\hat{\phi} \frac{\partial}{\partial \theta}-\hat{\theta} \frac{1}{\sin \theta} \frac{\partial}{\partial \phi}\right) \cos \theta\right] \times \hat{\mathbf{r}} \\
& =-i \sqrt{\frac{3}{8 \pi}}[\hat{\phi}(-\sin \theta)] \times \hat{\mathbf{r}} \\
& =i \sqrt{\frac{3}{8 \pi}} \sin \theta \hat{\theta}
\end{aligned}
$$

As a check, note that $\left|\mathbf{X}_{10} \times \mathbf{r}\right|^{2}=\frac{3}{8 \pi} \sin ^{2} \theta$ as on page 437 of [111], and illustrated in Fig. D.1. 
Now let's assume we have a detector set up that measures $\pi$-polarized light. Assuming this involves a lens capturing light from the radiating multipole, and recalling that we chose our magnetic field to be parallel to the $z$-axis, we should see light polarized along $\hat{\theta}$ as $\pi$-polarized. So,

$$
\begin{aligned}
\left(\mathbf{X}_{10}(\theta, \phi) \times \mathbf{r}\right) \cdot \hat{\theta} & =i \sqrt{\frac{3}{8 \pi}} \sin \theta \hat{\theta} \cdot \hat{\theta} \\
& =i \sqrt{\frac{3}{8 \pi}} \sin \theta
\end{aligned}
$$

which means the angular distribution of $\pi$-polarized photons is

$$
\left|\left(\mathbf{X}_{10} \times \mathbf{r}\right) \cdot \hat{\theta}\right|^{2}=\frac{3}{8 \pi} \sin ^{2} \theta
$$

Thus, if you are observing an $\mathbf{X}_{10}$ multipole with a perfect detector for $\pi$-polarized light, the probability you detect an emitted photon at a given angle is:

$$
\frac{\left|\left(\mathbf{X}_{10} \times \mathbf{r}\right) \cdot \hat{\theta}\right|^{2}}{\left|\mathbf{X}_{10} \times \mathbf{r}\right|^{2}}=\frac{\frac{3}{8 \pi} \sin ^{2} \theta}{\frac{3}{8 \pi} \sin ^{2} \theta}=1
$$

Of course, the number of photons you collect is determined by the radiation pattern. All the above states is that if you are looking for $\pi$-polarization, and you're looking at an $\mathbf{X}_{10}$ multipole, you'll see every photon reaching your detection optics, no matter what angle you view it from.

Next, we look at how much of the $\mathbf{X}_{10}$ radiation appears to be $\sigma^{+}$-polarized. While it may be correct to define $\sigma^{+}$-polarization as

$$
\begin{aligned}
\hat{\sigma^{+}}= & \frac{1}{\sqrt{2}}(\hat{\mathbf{x}}+i \hat{\mathbf{y}}) \\
= & \frac{1}{\sqrt{2}}(\sin \theta \cos \phi \hat{\mathbf{r}}+\cos \theta \cos \phi \hat{\theta}-\sin \phi \hat{\phi} \\
& +i \sin \theta \sin \phi \hat{\mathbf{r}}+i \cos \theta \sin \phi \hat{\theta}+i \cos \phi \hat{\phi}) \\
= & \frac{1}{\sqrt{2}}(\sin \theta[\cos \phi+i \sin \phi] \hat{\mathbf{r}}+\cos \theta[\cos \phi+i \sin \phi] \hat{\theta} \\
& -[\sin \phi-i \cos \phi] \hat{\phi})
\end{aligned}
$$


The two polarizations that we can distinguish in lab (using waveplates and polarizers) are $\sigma^{R}$ and $\sigma^{L}$. Only at $\theta=0$, when

$$
\hat{\sigma^{+}}=\frac{1}{\sqrt{2}}([\cos \phi+i \sin \phi] \hat{\theta}-[\sin \phi-i \cos \phi] \hat{\phi})=\frac{1}{\sqrt{2}}\left(e^{i \phi} \hat{\theta}+i e^{i \phi} \hat{\phi}\right)
$$

is it true that $\sigma^{R}=\sigma^{+}$. Here we define (by simple geometric arguments) that

$$
\hat{\sigma^{R}}=\frac{1}{\sqrt{2}}(\hat{\theta}+i \hat{\phi})
$$

which accurately defines the rotating electric field polarization at every angle, irrespective of $\phi$ (which is fine, as this is just an overall phase, and we end up taking the absolute value squared of everything in the end anyways). Given this, note that at $\theta=0, \sigma^{R}=\sigma^{+}$.

Similarly, we define $\sigma^{L}$-polarization as

$$
\hat{\sigma^{L}}=\frac{1}{\sqrt{2}}(\hat{\theta}-i \hat{\phi})
$$

by the same arguments given above.

Given the above, we now calculate the scalar product of $\sigma^{L}$ and $\mathbf{X}_{10}$.

$$
\begin{aligned}
\left(\mathbf{X}_{10} \times \hat{\mathbf{r}}\right) \cdot \hat{\sigma^{L}} & =\left(i \sqrt{\frac{3}{8 \pi}} \sin \theta \hat{\theta}\right) \frac{1}{\sqrt{2}}(\hat{\theta}+i \hat{\phi}) \\
& =i \sqrt{\frac{3}{16 \pi}} \sin \theta
\end{aligned}
$$

where in the first line of the previous equation, recall that the scalar product involves the complex conjugate of the second vector. The distribution of $\sigma^{L}$-polarized photons is then

$$
\left|\left(\mathbf{X}_{10} \times \hat{\mathbf{r}}\right) \cdot \hat{\sigma^{L}}\right|^{2}=\frac{3}{16 \pi} \sin ^{2} \theta
$$

and therefore the probability that a detector for $\sigma^{L}$-polarization will detect a photon emitted by $\mathbf{X}_{10}$ as a function of $\theta$ is

$$
\frac{\left|\left(\mathbf{X}_{10} \times \hat{\mathbf{r}}\right) \cdot \hat{\sigma^{L}}\right|^{2}}{\left|\mathbf{X}_{10} \times \mathbf{r}\right|^{2}}=\frac{\frac{3}{16 \pi} \sin ^{2} \theta}{\frac{3}{8 \pi} \sin ^{2} \theta}=\frac{1}{2}
$$


And this makes sense. If the thing you're observing only produces linearly polarized light, and you observe with something that only sees circular polarization, you're going to see exactly half of the light.

Clearly, the result for $\sigma^{R}$-polarization will be the same.

\section{D.2 Radiation from $\Delta \ell=1, \Delta m=1$ transitions}

Similar to the previous section, for $\ell=1, m=1$ we find

$$
\begin{aligned}
\mathbf{X}_{11}(\theta, \phi) \times \mathbf{r} & =\frac{1}{\sqrt{2}}\left[-i(\mathbf{r} \times \nabla) Y_{11}(\theta, \phi)\right] \times \hat{\mathbf{r}} \\
& =\frac{-i}{\sqrt{2}}\left[(\mathbf{r} \times \nabla)\left(-\sqrt{\frac{3}{8 \pi}} \sin \theta e^{i \phi}\right)\right] \times \hat{\mathbf{r}} \\
& =i \sqrt{\frac{3}{16 \pi}}\left(r \hat{\mathbf{r}} \times\left[\hat{\mathbf{r}} \frac{\partial}{\partial r}+\hat{\theta} \frac{1}{r} \frac{\partial}{\partial \theta}+\hat{\phi} \frac{1}{r \sin \theta} \frac{\partial}{\partial \phi}\right] \sin \theta e^{i \phi}\right) \times \hat{\mathbf{r}} \\
& =i \sqrt{\frac{3}{16 \pi}}\left[\left(\hat{\phi} \frac{\partial}{\partial \theta}-\hat{\theta} \frac{1}{\sin \theta} \frac{\partial}{\partial \phi}\right) \sin \theta e^{i \phi}\right] \times \hat{\mathbf{r}} \\
& =i e^{i \phi} \sqrt{\frac{3}{16 \pi}}[\cos \theta \hat{\phi}-i \hat{\theta}] \times \hat{\mathbf{r}} \\
& =i e^{i \phi} \sqrt{\frac{3}{16 \pi}}[\cos \theta \hat{\theta}+i \hat{\phi}]
\end{aligned}
$$

As a check, note that $\left|\mathbf{X}_{11} \times \mathbf{r}\right|^{2}=\frac{3}{16 \pi}\left(\cos ^{2} \theta+1\right)$, as on page 437 of [111], and illustrated in Fig. D.1.

Now we calculate the angular distribution of $\pi$-polarized light from $\mathbf{X}_{11}$ radiation.

$$
\begin{aligned}
\left(\mathbf{X}_{11} \times \mathbf{r}\right) \cdot \hat{\theta} & =i e^{i \phi} \sqrt{\frac{3}{16 \pi}}[\cos \theta \hat{\theta}+i \hat{\phi}] \cdot \hat{\theta} \\
& =i e^{i \phi} \sqrt{\frac{3}{16 \pi}} \cos \theta
\end{aligned}
$$

So the distribution of $\pi$-polarized photons is

$$
\left|\left(\mathbf{X}_{11} \times \mathbf{r}\right) \cdot \hat{\theta}\right|^{2}=\frac{3}{16 \pi} \cos ^{2} \theta
$$




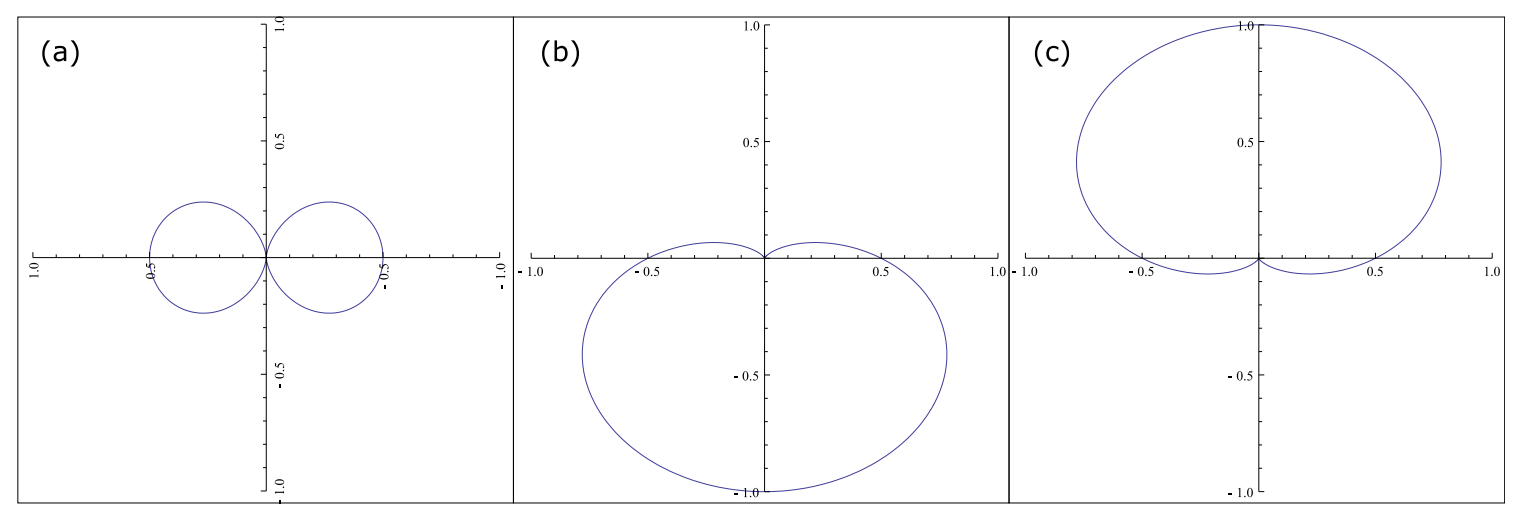

Figure D.2: The angular distribution of probability of detecting a photon emitted into $\mathbf{X}_{11}$ as (a) $\pi$-polarized, (b) $\sigma^{L}$-polarized, and (c) $\sigma^{R}$-polarized.

And therefore, the probability that you will detect a photon emitted from a $\mathbf{X}_{11}$ multipole with a detector for $\pi$-polarized light is given by

$$
\frac{\left|\left(\mathbf{X}_{11} \times \mathbf{r}\right) \cdot \hat{\theta}\right|^{2}}{\left|\mathbf{X}_{11} \times \mathbf{r}\right|^{2}}=\frac{\frac{3}{16 \pi} \cos ^{2} \theta}{\frac{3}{16 \pi}\left(\cos ^{2} \theta+1\right)}=\frac{\cos ^{2} \theta}{\cos ^{2} \theta+1}
$$

The result (Eq. D.18) is plotted in Fig. D.2(a). As shown, while looking perpendicular to the quantization ( $\mathrm{z}$ ) axis, zero photons will be detected. This because, at this angle, all of the light emitted by a $\mathbf{X}_{11}$ multipole should appear as linearly polarized in the $x-y$ plane, and therefore orthogonal to $\pi$-polarized light, which has polarization parallel to the $z$-axis. See, e.g. page 245 of Ref. [170].

To find the distribution of $\sigma^{L}$-polarization, we need to take the scalar product of the polarization vector with the electric multipole vector.

$$
\begin{aligned}
\left(\mathbf{X}_{11} \times \mathbf{r}\right) \cdot \hat{\sigma^{L}} & =i e^{i \phi} \sqrt{\frac{3}{16 \pi}}[\cos \theta \hat{\theta}+i \hat{\phi}] \cdot \hat{\sigma}^{L} \\
& =i e^{i \phi} \sqrt{\frac{3}{32 \pi}}[\cos \theta \hat{\theta}+i \hat{\phi}](\hat{\theta}-i \hat{\phi})^{*} \\
& =i e^{i \phi} \sqrt{\frac{3}{32 \pi}}[\cos \theta \hat{\theta}+i \hat{\phi}](\hat{\theta}+i \hat{\phi}) \\
& =i e^{i \phi} \sqrt{\frac{3}{32 \pi}}[\cos \theta-1]
\end{aligned}
$$


The angular distribution of $\sigma^{L}$-polarized photons emitted by $\mathbf{X}_{11}$ is then

$$
\left|\left(\mathbf{X}_{11} \times \mathbf{r}\right) \cdot \hat{\sigma^{L}}\right|^{2}=\frac{3}{32 \pi}\left(\cos ^{2} \theta-2 \cos \theta+1\right)
$$

Therefore, the probability that a photon emitted by a $\mathbf{X}_{11}$ multipole will be detected as having $\sigma^{L}$-polarization is given by

$$
\frac{\left|\left(\mathbf{X}_{11} \times \mathbf{r}\right) \cdot \hat{\sigma}^{L}\right|^{2}}{\left|\mathbf{X}_{11} \times \mathbf{r}\right|^{2}}=\frac{\frac{3}{32 \pi}\left(\cos ^{2} \theta-2 \cos \theta+1\right)}{\frac{3}{16 \pi}\left(\cos ^{2} \theta+1\right)}=\frac{\left(\cos ^{2} \theta-2 \cos \theta+1\right)}{2\left(\cos ^{2} \theta+1\right)}
$$

The angular distribution of Eq. D.21 is shown in Fig. D.2(b). As shown, in the $x-y$ plane, half of the photons emitted into $\mathbf{X}_{11}$ are detected as being $\sigma^{L}$-polarized, but no $\sigma^{L}$-polarized photons are seen along the $+z$-axis.

Lastly, we delve into a final calculation to reveal the extent of $\sigma^{R}$-polarization detected from $\mathbf{X}_{11}$ radiation.

$$
\begin{aligned}
\left(\mathbf{X}_{11} \times \hat{\mathbf{r}}\right) \cdot \hat{\sigma^{R}} & =i e \sqrt{\frac{3}{16 \pi}}(\cos \theta \hat{\theta}+i \hat{\phi}) \cdot \hat{\sigma^{R}} \\
& =i e \sqrt{\frac{3}{32 \pi}}(\cos \theta \hat{\theta}+i \hat{\phi})(\hat{\theta}+i \hat{\phi})^{*} \\
& =i e^{i \phi} \sqrt{\frac{3}{32 \pi}}(\cos \theta \hat{\theta}+i \hat{\phi})(\hat{\theta}-i \hat{\phi}) \\
& =i e^{i \phi} \sqrt{\frac{3}{32 \pi}}(\cos \theta+1)
\end{aligned}
$$

The angular distribution of $\sigma^{R}$-polarized photons is then

$$
\left|\left(\mathbf{X}_{11} \times \hat{\mathbf{r}}\right) \cdot \hat{\sigma^{R}}\right|^{2}=\frac{3}{32 \pi}\left(\cos ^{2} \theta+2 \cos \theta+1\right)
$$

and the probability of detecting a photon emitted by a $\mathbf{X}_{11}$ multipole as $\sigma^{R}$-polarized is given by

$$
\frac{\left|\left(\mathbf{X}_{11} \times \hat{\mathbf{r}}\right) \cdot \hat{\sigma^{R}}\right|^{2}}{\left|\mathbf{X}_{11} \times \mathbf{r}\right|^{2}}=\frac{\frac{3}{32 \pi}\left(\cos ^{2} \theta+2 \cos \theta+1\right)}{\frac{3}{16 \pi}\left(\cos ^{2} \theta+1\right)}=\frac{\left(\cos ^{2} \theta+2 \cos \theta+1\right)}{2\left(\cos ^{2} \theta+1\right)}
$$


The plot of Eq. D.24 is shown in Fig. D.2(c). As is expected, when viewed along the quantization $(+z)$ axis, all of the emitted photons are seen as $\sigma^{R}$-polarized; here, $\sigma^{R}=\sigma^{+}$. However, in the $x-y$ plane, $50 \%$ of the time the photons are measured to be $\sigma^{L}$-polarized, and $50 \%$ of the time they are measured as $\sigma^{R}$. The reason behind this is that the light emitted by a $\mathbf{X}_{11}$ multipole appears linear when viewed perpendicular to the quantization axis (see, e.g. page 245 of [170]). Finally, when looking along the $-z$-axis, we see $\sigma^{L}$ only, because in this direction $\sigma^{+}=\sigma^{L}$.

\section{D.3 Implications for Polarization-Encoded Photons}

Using the knowledge gained in the previous sections, since we know the numerical aperature of our collection optics, we can calculate the error in the experiment due to polarization mixing. While taking measurements in the recent entanglement experiment utilizing polarization-encoded photons [145], we could distinguish between $\sigma^{R}$ and $\sigma^{L}$ photons. However, in the experiment, we want to be able to equate a right-handed circularly polarized photon with $\Delta m=1$; in other words, we assume every right-handed circularly polarized photon we detect was a $\sigma^{+}$-polarized photon. From the calculations of the previous section, we know that this is not completely true, so let's find out the error.

The question is: if we measure a $\sigma^{R}$ photon, what is the probability it was emitted by either $\mathbf{X}_{1-1}$ or $\mathbf{X}_{10}$ ?

To calculate this, we simply need to add up (integrate) all of the $\sigma^{R}$ photons from $\mathbf{X}_{1-1}$ and $\mathbf{X}_{10}$, and divide by the total number of $\sigma^{R}$ photons. For $\mathbf{X}_{1-1}$, the number of $\sigma^{R}$ photons is proportional to the integral of Eq. D.20 over the solid angle subtended by our optics (although we never calculated $\mathbf{X}_{1-1}$ explicitly, it should be clear that the results are just the mirror of $\mathbf{X}_{11}$ ). Similarly, the number of $\sigma^{R}$ photons 
detected from $\mathbf{X}_{10}$ and $\mathbf{X}_{11}$ will be given by integrating Eq. D.13 and Eq. D.23, respectively. Therefore, the total error in the polarization-encoded photons experiment due to polarization mixing is given by

$$
\text { Error }_{\sigma^{+}}=\frac{\int_{0}^{\alpha}\left(\left|\left(\mathbf{X}_{1-1} \times \hat{\mathbf{r}}\right) \cdot \hat{\sigma^{R}}\right|^{2}+\left|\left(\mathbf{X}_{10} \times \hat{\mathbf{r}}\right) \cdot \hat{\sigma^{R}}\right|^{2}\right) \sin \theta d \theta}{\int_{0}^{\alpha}\left(\left|\left(\mathbf{X}_{11} \times \hat{\mathbf{r}}\right) \cdot \hat{\sigma^{R}}\right|^{2}+\left|\left(\mathbf{X}_{1-1} \times \hat{\mathbf{r}}\right) \cdot \hat{\sigma^{R}}\right|^{2}+\left|\left(\mathbf{X}_{10} \times \hat{\mathbf{r}}\right) \cdot \hat{\sigma}^{R}\right|^{2}\right) \sin \theta d \theta}
$$

Calculating these integrals, for $\mathbf{X}_{1-1}$ we get

$$
\begin{aligned}
2(2 \pi) \int_{0}^{\alpha}\left|\left(\mathbf{X}_{1-1} \times \hat{\mathbf{r}}\right) \cdot \hat{\sigma^{R}}\right|^{2} \sin \theta d \theta= & 4 \pi \frac{3}{32 \pi} \int_{0}^{\alpha}\left(\cos ^{2} \theta-2 \cos \theta+1\right) \sin \theta d \theta \\
= & \frac{3}{8} \int_{0}^{\alpha}(\cos \theta-1)^{2} \sin \theta d \theta \\
& (\operatorname{letting} u=\cos \theta-1, d u=-\sin \theta d \theta) \\
= & -\frac{3}{8} \int_{0}^{\cos \alpha-1} u^{2} d u \\
= & -\frac{3}{8}\left[\frac{1}{3} u^{3}\right]_{0}^{\cos \alpha-1} \\
= & \frac{1}{8}(1-\cos \alpha)^{3}
\end{aligned}
$$

And for $\mathbf{X}_{10}$ we have

$$
\begin{aligned}
2(2 \pi) \int_{0}^{\alpha}\left|\left(\mathbf{X}_{10} \times \hat{\mathbf{r}}\right) \cdot \hat{\sigma^{R}}\right|^{2} \sin \theta d \theta & =4 \pi \frac{3}{16 \pi} \int_{0}^{\alpha} \sin ^{2} \theta \sin \theta d \theta \\
& =\frac{3}{4} \int_{0}^{\alpha}\left(1-\cos ^{2} \theta\right) \sin \theta d \theta \\
& =\frac{3}{4} \int_{0}^{\alpha} \sin \theta d \theta-\frac{3}{4} \int_{0}^{\alpha} \cos ^{2} \theta \sin \theta d \theta \\
& =\frac{3}{4}-\frac{3}{4} \cos \alpha+\frac{3}{4}\left(\frac{1}{3} \cos ^{3} \alpha-\frac{1}{3}\right) \\
& =\frac{1}{4}\left(2-3 \cos \alpha+\cos ^{3} \alpha\right)
\end{aligned}
$$



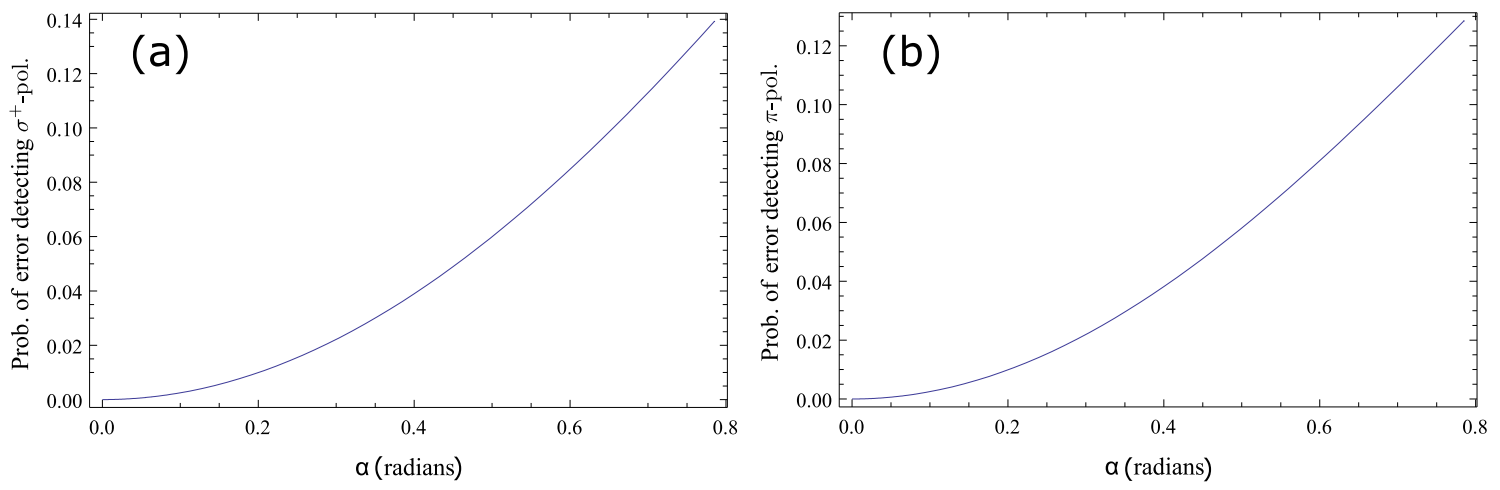

Figure D.3: Error in the discrimination of photon polarizations. (a) The probability that a photon measured as $\sigma^{R}\left(\sigma^{+}\right.$, assumed to come from $\left.\mathbf{X}_{11}\right)$ was emitted by $\mathbf{X}_{1-1}$ or $\mathbf{X}_{10}$ as a function of $1 / 2$ the angle subtended by the observation optics, $\alpha$. (b) The probability that a photon measured as linear along the magnetic field $(\pi$, assumed to come from $\mathbf{X}_{10}$ ) was emitted by $\mathbf{X}_{1-1}$ or $\mathbf{X}_{11}$.

And, finally, for $\mathbf{X}_{11}$ we find

$$
\begin{aligned}
2(2 \pi) \int_{0}^{\alpha}\left|\left(\mathbf{X}_{11} \times \hat{\mathbf{r}}\right) \cdot \hat{\sigma}^{R}\right|^{2} \sin \theta d \theta= & 4 \pi \frac{3}{32 \pi} \int_{0}^{\alpha}\left(\cos ^{2} \theta+2 \cos \theta+1\right) \sin \theta d \theta \\
& (\text { letting } u=\cos \theta) \\
= & -\frac{3}{8} \int_{1}^{\cos \alpha}\left(u^{2}+2 u+1\right) d u \\
= & -\frac{3}{8}\left[\frac{1}{3} u^{3}+u^{2}+u\right]_{1}^{\cos \alpha} \\
= & -\frac{3}{8}\left(\frac{1}{3} \cos ^{3} \alpha+\cos ^{2} \alpha+\cos \alpha-\frac{1}{3}-1-1\right) \\
= & \frac{1}{8}\left(7-3 \cos \alpha-3 \cos ^{2} \alpha-\cos ^{3} \alpha\right) \quad(\mathrm{D} .28)
\end{aligned}
$$

Plugging Eqs. D.26, D.27, and D.28 into Eq. D.25, we get

$$
\text { Error }_{\sigma^{+}}=\frac{5-9 \cos \alpha+3 \cos ^{2} \alpha+\cos ^{3} \alpha}{12-12 \cos \alpha}
$$

which is plotted in Fig. D.3(a).

Thus, given a numerical aperature of 0.23 (the CVI triplet), $\alpha=\arcsin (0.23)=$ 0.232, we get Error $_{\sigma^{+}}(0.232)=1.33 \%$. In Fig. D.3, Error $_{\sigma^{+}}(\alpha)$ is shown all the way out to $\pi / 4$. As is seen from the graph, the error hits $1 \%$ around $\alpha \approx 0.2$. 


\section{D.4 Implications for Frequency-Encoded Photons}

In the quantum gate experiment, we will want to detect $\pi$-polarized light, and therefore set the observation axis at $\theta=\frac{\pi}{2}$. Thus, we repeat the calculation of the previous section for this configuration.

The error in this case will be:

$$
\begin{aligned}
\text { Error }_{\pi}= & {\left[4 \int _ { \pi / 2 } ^ { \pi / 2 + \alpha } \int _ { 0 } ^ { \alpha \operatorname { c o s } ( \operatorname { a r c s i n } ( \frac { 1 } { \alpha } ( \frac { \pi } { 2 } - \theta ) ) ) } \left(\left|\left(\mathbf{X}_{1-1} \times \hat{\mathbf{r}}\right) \cdot \hat{\theta}\right|^{2}\right.\right.} \\
& \left.\left.+\left|\left(\mathbf{X}_{11} \times \hat{\mathbf{r}}\right) \cdot \hat{\theta}\right|^{2}\right) \sin \theta d \theta d \phi\right] \\
\div & {\left[4 \int _ { \pi / 2 } ^ { \pi / 2 + \alpha } \int _ { 0 } ^ { \alpha \operatorname { c o s } ( \operatorname { a r c s i n } ( \frac { 1 } { \alpha } ( \frac { \pi } { 2 } - \theta ) ) ) } \left(\left|\left(\mathbf{X}_{1-1} \times \hat{\mathbf{r}}\right) \cdot \hat{\theta}\right|^{2}\right.\right.} \\
& \left.\left.+\left|\left(\mathbf{X}_{10} \times \hat{\mathbf{r}}\right) \cdot \hat{\theta}\right|^{2}+\left|\left(\mathbf{X}_{11} \times \hat{\mathbf{r}}\right) \cdot \hat{\theta}\right|^{2}\right) \sin \theta d \theta d \phi\right]
\end{aligned}
$$

A numerical integration of the above equation is plotted in Fig. D.3(b). Hence, for the CVI triplet lens (numerical aperture 0.23 ), the error in polarization discrimination will again be approximately $1.3 \%$.

\section{D.5 Other Considerations}

The calculations above are done for photons in freespace. However, in the actual experiment we couple the spontaneously emitted photons into a single-mode optical fiber. At particular angles of observation with respect to the quantization axis, light from some radiation patterns will not couple into the single-mode fiber. Therefore, the results presented above should be viewed as an upper bound on the expected contribution of error due to polarization-mixing. 


\section{APPENDIX E}

\section{Phase of the Gate}

In Chap. VII and VIII, we ignored the phase evolution of the two-qubit state in the implementation of the gate. Here, we include this phase evolution, showing that the final state of the two ions in contingent only upon the initial states of the two ions and the path length of the photons to the beamsplitter. Moreover, the path length dependance is sensitive only on the scale of the frequency difference of the two photonic frequency states: $14.7 \mathrm{GHz}$. Stability on this scale $(2 \mathrm{~cm})$ is easily achieved.

After state preparation, at time $t=0$, the state of the two ion system is simply:

$$
|\psi(t=0)\rangle_{i o n s}=\left(\alpha|0\rangle_{a}+\beta|1\rangle_{a}\right) \otimes\left(\gamma|0\rangle_{b}+\delta|1\rangle_{b}\right)
$$

In order to keep tabs on all the phases involved, we assume atom $a$ is excited at time $t_{e a}$ and atom $b$ is excited at time $t_{e b}$, so that quantum state after excitation is

$$
\begin{aligned}
\left|\psi\left(t>t_{e a}, t_{e b}\right)\right\rangle_{i o n s}= & \left(\alpha e^{-i \omega_{p}\left(t-t_{e a}\right)}\left|0^{\prime}\right\rangle_{a}+\beta e^{-i \omega_{s} t_{e a}}\left|1^{\prime}\right\rangle_{a}\right) \\
& \otimes\left(\gamma e^{-i \omega_{p}\left(t-t_{e b}\right)}\left|0^{\prime}\right\rangle_{b}+\delta e^{-i \omega_{s} t_{e b}}\left|1^{\prime}\right\rangle_{b}\right)
\end{aligned}
$$

The additional phase factors are simply from the state evolution between the two

states, where we have written the energy splitting of the ${ }^{2} S_{1 / 2}$ hyperfine states as 
$\omega_{s}=2 \pi \times 12.6 \mathrm{GHz}$ and the ${ }^{2} P_{1 / 2}$ hyperfine levels as $\omega_{p}=2 \pi \times 2.1 \mathrm{GHz} .{ }^{1}$ We have not made any assumptions on the time ordering of $t_{e a}$ and $t_{e b}$.

If at time $t_{p a}\left(t_{p b}\right)$ ion $a(b)$ emits a $\pi$-polarized photon while returning to the ${ }^{2} S_{1 / 2}$ ground state, then then state of the system at time $t>t_{p a}, t_{p b}$ is:

$$
\begin{aligned}
\left|\psi\left(t>t_{p a}, t_{p b}\right)\right\rangle_{i o n s, p h}= & \left(\alpha e^{-i \omega_{p}\left(t_{p a}-t_{e a}\right)}|0\rangle_{a} e^{i\left(k_{b l u e} x_{a}-\left(\omega_{\text {red }}+\omega_{s}+\omega_{p}\right)\left(t-t_{p a}\right)\right)}\left|\nu_{\text {blue }}\right\rangle_{a}\right. \\
& \left.+\beta e^{-i \omega_{s}\left(t-t_{p a}+t_{e a}\right)}|1\rangle_{a} e^{i\left(k_{\text {red }} x_{a}-\omega_{\text {red }}\left(t-t_{p a}\right)\right)}\left|\nu_{\text {red }}\right\rangle_{a}\right) \\
& \otimes\left(\gamma e^{-i \omega_{p}\left(t_{p b}-t_{e b}\right)}|0\rangle_{b} e^{i\left(k_{\text {blue }} x_{b}-\left(\omega_{\text {red }}+\omega_{s}+\omega_{p}\right)\left(t-t_{p b}\right)\right)}\left|\nu_{\text {blue }}\right\rangle_{b}\right. \\
& \left.+\delta e^{-i \omega_{s}\left(t-t_{p b}+t_{e b}\right)}|1\rangle_{b} e^{i\left(k_{\text {red }} x_{b}-\omega_{\text {red }}\left(t-t_{p b}\right)\right)}\left|\nu_{\text {red }}\right\rangle_{b}\right)
\end{aligned}
$$

where we have defined the optical frequency of the ${ }^{2} P_{1 / 2}\left|F=0, m_{F}=0\right\rangle$ to ${ }^{2} S_{1 / 2}$ $\left|F=1, m_{F}=0\right\rangle$ transition as $\omega_{r e d}=2 \pi c /(369.5 \mathrm{~nm})=2 \pi \times 811 \mathrm{THz} ; k_{\text {red }}=\omega_{\text {red }} / c ;$ $k_{\text {blue }}=\left(\omega_{\text {red }}+\omega_{s}+\omega_{p}\right) / c$; and $x_{a}, x_{b}$ as the (time-dependent) distance traversed by photons $a, b$, respectively.

\footnotetext{
${ }^{1}$ In writing the phases this way, we have assumed the phase evolution during the excitation pulse is negligible. Since the duration of the pulse is much less than the periods in the phase factors, this is justified.
} 
Gathering terms of equal phase rotation, we can rewrite Eq. E.3 as:

$$
\begin{aligned}
& \left|\psi\left(t>t_{p a}, t_{p b}\right)\right\rangle_{i o n s, p h}=\left(\alpha e^{i \omega_{p}\left(t_{e a}-t\right)} e^{i \omega_{\text {red }}\left(t_{p a}-t\right)} e^{i \omega_{s}\left(t_{p a}-t\right)} e^{i k_{b l u e} x_{a}}|0\rangle_{a}\left|\nu_{b l u e}\right\rangle_{a}\right. \\
& \left.+\beta e^{i \omega_{s}\left(t_{p a}-t-t_{e a}\right)} e^{i \omega_{r e d}\left(t_{p a}-t\right)} e^{i k_{r e d} x_{a}}|1\rangle_{a}\left|\nu_{r e d}\right\rangle_{a}\right) \\
& \otimes\left(\gamma e^{i \omega_{p}\left(t_{e b}-t\right)} e^{i \omega_{\text {red }}\left(t_{p b}-t\right)} e^{i \omega_{s}\left(t_{p b}-t\right)} e^{i k_{\text {blue }} x_{b}}|0\rangle_{b}\left|\nu_{b l u e}\right\rangle_{b}\right. \\
& \left.+\delta e^{i \omega_{s}\left(t_{p b}-t-t_{e b}\right)} e^{i \omega_{r e d}\left(t_{p b}-t\right)} e^{i k_{r e d} x_{b}}|1\rangle_{b}\left|\nu_{r e d}\right\rangle_{b}\right) \\
& =e^{i k_{r e d} x_{a}} e^{i \omega_{r e d}\left(t_{p a}-t\right)} e^{i \omega_{s}\left(t_{p a}-t\right)} \\
& \times\left(\alpha e^{i \omega_{p}\left(t_{e a}-t\right)} e^{i\left(k_{b l u e}-k_{r e d}\right) x_{a}}|0\rangle_{a}\left|\nu_{\text {blue }}\right\rangle_{a}\right. \\
& \left.+\beta e^{-i \omega_{s} t_{e a}}|1\rangle_{a}\left|\nu_{r e d}\right\rangle_{a}\right) \\
& \otimes e^{i k_{r e d} x_{b}} e^{i \omega_{r e d}\left(t_{p b}-t\right)} e^{i \omega_{s}\left(t_{p b}-t\right)} \\
& \times\left(\gamma e^{i \omega_{p}\left(t_{e b}-t\right)} e^{i\left(k_{b l u e}-k_{r e d}\right) x_{b}}|0\rangle_{b}\left|\nu_{b l u e}\right\rangle_{b}\right. \\
& \left.+\delta e^{-i \omega_{s} t_{e b}}|1\rangle_{b}\left|\nu_{r e d}\right\rangle_{b}\right)
\end{aligned}
$$

Dropping all global phases, we can then write the state of the system as:

$$
\begin{aligned}
\left|\psi\left(t>t_{p a}, t_{p b}\right)\right\rangle_{i o n s, p h}= & \left(\alpha e^{i \omega_{p}\left(t_{e a}-t\right)} e^{i\left(k_{\text {blue }}-k_{\text {red }}\right) x_{a}}|0\rangle_{a}\left|\nu_{\text {blue }}\right\rangle_{a}\right. \\
& \left.+\beta e^{-i \omega_{s} t_{e a}}|1\rangle_{a}\left|\nu_{\text {red }}\right\rangle_{a}\right) \\
\otimes & \left(\gamma e^{i \omega_{p}\left(t_{e b}-t\right)} e^{i\left(k_{\text {blue }}-k_{\text {red }}\right) x_{b}}|0\rangle_{b}\left|\nu_{b l u e}\right\rangle_{b}\right. \\
& \left.+\delta e^{-i \omega_{s} t_{e b}}|1\rangle_{b}\left|\nu_{\text {red }}\right\rangle_{b}\right) \\
= & \alpha \gamma e^{i \omega_{p}\left(t_{e a}+t_{e b}-2 t\right)} e^{i\left(k_{b l u e}-k_{\text {red }}\right)\left(x_{a}+x_{b}\right)}|0\rangle_{a}|0\rangle_{b}\left|\nu_{b l u e}\right\rangle_{a}\left|\nu_{b l u e}\right\rangle_{b} \\
& +\alpha \delta e^{i \omega_{p}\left(t_{e a}-t\right)} e^{-i \omega_{s} t_{e b}} e^{i\left(k_{b l u e}-k_{\text {red }}\right) x_{a}}|0\rangle_{a}|1\rangle_{b}\left|\nu_{b l u e}\right\rangle_{a}\left|\nu_{\text {red }}\right\rangle_{b} \\
& +\beta \gamma e^{i \omega_{p}\left(t_{e b}-t\right)} e^{-i \omega_{s} t_{e a}} e^{i\left(k_{b l u e}-k_{\text {red }}\right) x_{b}}|1\rangle_{a}|0\rangle_{b}\left|\nu_{\text {red }}\right\rangle_{a}\left|\nu_{b l u e}\right\rangle_{b} \\
& +\beta \delta e^{-i \omega_{s}\left(t_{e a}+t_{e b}\right)}|1\rangle_{a}|1\rangle_{b}\left|\nu_{\text {red }}\right\rangle_{a}\left|\nu_{\text {red }}\right\rangle_{b}
\end{aligned}
$$

As we saw in Chap. VII, ideally, only the $\left|\psi^{-}\right\rangle_{p h}$ state of the photons will yield coincident detections. Of course, if there is temporal mismatch of the photon wavepackets 
at the beamsplitter then the resulting state of the two-ion system will have other contributions that will degrade the overall fidelity of the process. Since the contribution of these other elements is approximately $1-e^{-\Delta t / \tau}$, and we have arranged for the photon wavepackets to arrive at the beamsplitter within 100 ps of each other (so $1-e^{-0.1 / 8.12} \approx 0.01$ ), for the purpose of determining the phase of the entangled state we ignore the contribution of this temporal mismatch.

We will write $t=t_{B S}$ as the time the photons are incident at the beamsplitter. After the beamsplitter, each photon is in a superposition of the two output modes of the beamsplitter, and so the phases acquired by each photon term after the beamsplitter are equal. As such, we can ignore the propagation after the beamsplitter, and just assume the two photons are measured immediately upon exiting the beamsplitter $\left(\right.$ at $\left.t=t_{B S}\right)$. Thus, the state the ions are projected into following coincident detection of the two photons is:

$$
\begin{aligned}
\left|\psi\left(t>t_{B S}\right)\right\rangle_{\text {ions }}= & { }_{p h}\left\langle\psi^{-} \mid \psi(t)\right\rangle_{\text {ions }, p h} \\
= & \alpha \delta e^{i \omega_{p}\left(t_{e a}-t\right)} e^{-i \omega_{s} t_{e b}} e^{i\left(k_{b l u e}-k_{\text {red }}\right) x_{0 a}}|0\rangle_{a}|1\rangle_{b} \\
& -\beta \gamma e^{i \omega_{p}\left(t_{e b}-t\right)} e^{-i \omega_{s} t_{e a}} e^{i\left(k_{b l u e}-k_{\text {red }}\right) x_{0 b}}|1\rangle_{a}|0\rangle_{b} \\
= & \alpha \delta|0\rangle_{a}|1\rangle_{b}-\beta \gamma e^{i\left(\omega_{p}+\omega_{s}\right)\left(t_{e b}-t_{e a}\right)} e^{i\left(k_{b l u e}-k_{\text {red }}\right)\left(x_{0 b}-x_{0 a}\right)}|1\rangle_{a}|0\rangle_{b} \\
= & \alpha \delta|0\rangle_{a}|1\rangle_{b}-\beta \gamma e^{i c \Delta k\left(t_{e b}-t_{e a}\right)} e^{i \Delta k \Delta x}|1\rangle_{a}|0\rangle_{b}
\end{aligned}
$$

where we have again dropped global phase factors. Here, $x_{0 a}, x_{0 b}$ are the total path length from ion $a, b$ to the beamsplitter, respectively; $\Delta x=x_{0 b}-x_{0 a}$ is the difference of the two path lengths; and $\Delta k=k_{b l u e}-k_{\text {red }}=2 \pi /(2 \mathrm{~cm})$ is the difference in the wavenumbers.

Thus, we see that the phase of the final entangled state depends only upon the difference in excitation times of the two ions and the difference in the path lengths to 
the beamsplitter. Moreover, the phase acquired as a result of these disparities goes as $\Delta k=k_{\text {blue }}-k_{\text {red }}=2 \pi /(2 \mathrm{~cm})$, so that passively stability of the phase is easily achieved.

In the ideal case, the two photon wavepackets are incident on the beamsplitter at the same time $\left(t_{B S}\right)$, in which case:

$$
\begin{aligned}
c\left(t_{B S}-t_{e a}\right) & =x_{0 a} \\
c\left(t_{B S}-t_{e b}\right) & =x_{0 b} \\
\Rightarrow \Delta x=x_{0 a}-x_{0 b} & =c\left(t_{e a}-t_{e b}\right)
\end{aligned}
$$

in which case the argument of the exponential is zero, and the final entangled state is:

$$
\left|\psi\left(t>t_{B S}\right)\right\rangle_{i o n s}=\alpha \delta|0\rangle_{a}|1\rangle_{b}-\beta \gamma|1\rangle_{a}|0\rangle_{b}
$$

as expected. In the lab, though, our preliminary calibration measurement only allows us to determine the photon wavepackets arrive at the beamsplitter within 100 ps of each other. Since the success of the heralded quantum gate depends critically on knowledge of this phase, a more precise calibration is performed by repeatedly performing the heralded gate on a given input state (here $\alpha=\beta=\gamma=\delta=1 / \sqrt{2}$ ) and implementing an additional microwave $\pi / 2$ rotation on each ion before detection. The phase of this second microwave pulse is varied (with respect to the initial pulse phase) and the probability of detecting $|01\rangle$ or $|10\rangle$ at each phase shift measured. As shown in Fig. E.1, a sinusoidal fit to these measurements allow us to determine the phase of the entangled state. 


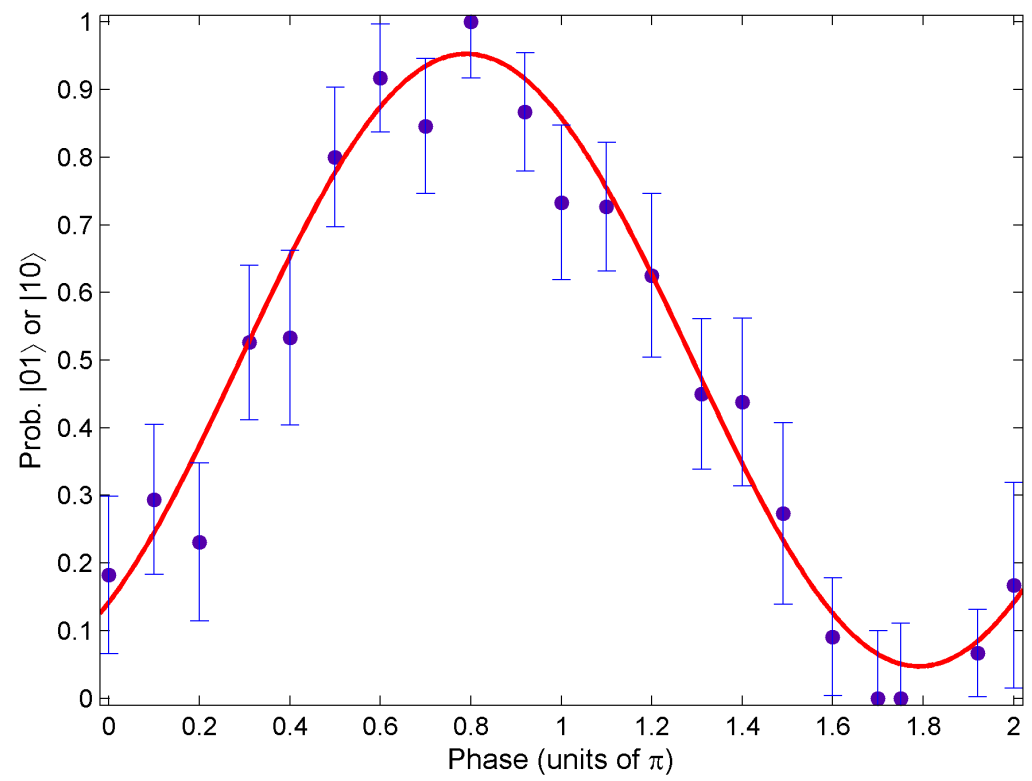

Figure E.1: Measured phase of the entangled state of the two ions after implementation of the quantum gate. Plotted is the probability of finding the two ions in the state $|01\rangle$ or $|10\rangle$ versus the phase of the second (measurement) microwave pulse. The data is a total of 290 events taken over a total of about 60 hours of measurment. 


\section{BIBLIOGRAPHY}




\section{BIBLIOGRAPHY}

[1] R. P. Feynman, "Simulating physics with computers", International Journal of Theoretical Physics 21, 467 (1982).

[2] D. Deutsch, "Quantum theory, the Church-Turing principle and the universal quantum computer", Proc. Royal Society of London A 400, 97 (1985).

[3] P. Shor, "Algorithms for quantum computation: discrete logarithms and factoring", in Proc. 35th Ann. Sym. Found. Comp. Sci., page 124, 1994.

[4] C. H. Bennett and G. Brassard, "Quantum cryptography: Public-key distribution and coin tossing", in Proceedings of IEEE International Conference on Computers, Systems and Signal Processing, Bangalore, India, page 175, IEEE Press, 1984.

[5] A. K. Ekert, "Quantum Cryptography Based on Bell's Theorem", Phys. Rev. Lett. 67, 661 (1991).

[6] W. K. Wootters and W. H. Zurek, "A single quantum cannot be cloned", Nature 299, 802 (1982).

[7] C. H. Bennett et al., "Teleporting an unknown quantum state via dual classical and EinsteinPodolsky-Rosen channels", Phys. Rev. Lett. 70, 1895 (1993).

[8] P. Kok et al., "Linear optical quantum computing with photonic qubits", Rev. Mod. Phys. 79, 135 (2007).

[9] R. Blatt and D. J. Wineland, "Entangled states of trapped atomic ions", Nature 453, 1008 (2008).

[10] J. Clarke and F. K. Wilhelm, "Superconducting quantum bits", Nature 453, 1031 (2008).

[11] H. J. Kimble, "The quantum internet", Nature 453, 1023 (2008).

[12] I. Bloch, "Quantum coherence and entanglement with ultracold atoms in optical lattices", Nature 453, 1016 (2008).

[13] B. B. Blinov, D. L. Moehring, L.-M. Duan, and C. Monroe, "Observation of entanglement between a single trapped atom and a single photon", Nature 428, 153 (2004).

[14] D. N. Matsukevich and A. Kuzmich, "Quantum State Transfer Between Matter and Light", Science 306, 663 (2004).

[15] J. Volz et al., "Observation of Entanglement of a Single Photon with a Trapped Atom", Phys. Rev. Lett. 96, 030404 (2006).

[16] H. Häffner et al., "Scalable multiparticle entanglement of trapped ions", Nature 438, 643 (2005).

[17] D. Leibfried et al., "Creation of a six-atom 'Schrödinger cat' state", Nature 438, 639 (2005). 
[18] D. Kielpinski, C. R. Monroe, and D. J. Wineland, "Architecture for a Large-Scale Ion-Trap Quantum Computer", Nature 417, 709 (2002).

[19] D. Stick et al., "Ion Trap in a Semiconductor Chip", Nature Physics 2, 36 (2006).

[20] S. Seidelin et al., "A microfabricated surface-electrode ion trap for scalable quantum information processing", Phys. Rev. Lett. 96, 253003 (2006).

[21] W. K. Hensinger et al., "T-junction ion trap array for two-dimensional ion shuttling, storage and manipulation", App. Phys. Lett. 88, 034101 (2006).

[22] R. B. Blakestad et al., "High fidelity transport of trapped-ion qubits through an X-junction trap array", (2009), arXiv:0901.0533.

[23] Q. A. Turchette et al., "Heating of trapped ions from the quantum ground state", Phys. Rev. A 61, 063418 (2000).

[24] L. Deslauriers et al., "Scaling and Suppression of Anomalous Heating in Ion Traps", Phys. Rev. Lett. 97, 103007 (2006).

[25] J. Labaziewicz et al., "Suppression of Heating Rates in Cryogenic Surface-Electrode Ion Traps", Phys. Rev. Lett. 100, 013001 (2008).

[26] R. Ursin et al., "Entanglement-based quantum communication over 144 km", Nature Physics 3, 481 (2007).

[27] H.-J. Briegel, W. Dür, J. I. Cirac, and P. Zoller, "Quantum Repeaters: The Role of Imperfect Local Operations in Quantum Communication", Phys. Rev. Lett. 81, 5932 (1998).

[28] L.-M. Duan, M. D. Lukin, J. I. Cirac, and P. Zoller, "Long-distance quantum communication with atomic ensembles and linear optics", Nature 414, 413 (2001).

[29] D. P. DiVincenzo, "The Physical Implementation of Quantum Computation", Fortschr. Phys. 48, 771 (2000).

[30] A. Barenco et al., "Elementary gates for quantum computation", Phys. Rev. A 52, 3457 (1995).

[31] M. A. Nielsen and I. L. Chuang, Quantum Computation and Quantum Information, Cambridge University Press, 2000.

[32] W. Paul, "Electromagnetic traps for charged and neutral particles", Rev. Mod. Phys. 62, 531 (1990).

[33] D. J. Wineland, R. E. Drullinger, and F. L. Walls, "Radiation Pressure Cooling of Bound Resonant Absorbers", Phys. Rev. Lett. 40, 1639 (1978).

[34] W. Neuhauser, M. Hohenstatt, P. Toschek, and H. Dehmelt, "Optical-Sideband Cooling of Visible Atom Cloud Confined in Parabolic Well", Phys. Rev. Lett. 41, 233 (1978).

[35] P. T. H. Fisk, M. J. Sellars, M. A. Lawn, and C. Coles, "Accurate Measurement of the 12.6 $\mathrm{GHz}$ "Clock" Transition in Trapped ${ }^{171} \mathrm{Yb}^{+}$Ions", IEEE Trans. Ultrasonics, Ferroelectrics, and Frequency Control 44, 344 (1997).

[36] R. E. March, "Quadrupole ion trap mass spectrometry: theory, simulation, recent developments and applications", Rapid Comm. in Mass Spect. 12, 1543 (1998).

[37] T. M. Fortier et al., "Precision atomic spectroscopy for improved limits on variation of the fine structure constant and local position invariance", Phys. Rev. Lett. 98, 070801 (2007). 
[38] T. Rosenband et al., "Frequency ratio of $\mathrm{Al}^{+}$and $\mathrm{Hg}^{+}$single-ion optical clocks; Metrology at the 17th decimal place", Science 319, 1808 (2008).

[39] D. J. Griffiths, Introduction to Electrodynamics, Prentice Hall, 3rd edition, 1999.

[40] H. G. Dehmelt, "Radiofrequency spectroscopy of stored ions I: storage", Adv. At. Mol. Phys. 3, 53 (1967).

[41] D. J. Wineland et al., "Experimental Issues in Coherent Quantum-State Manipulation of Trapped Atomic Ions", Journal of Research of the National Institute of Standards and Technology 103, 259 (1998).

[42] N. W. McLachlan, Theory and Application of Mathieu Functions, Dover Publications, Inc., 1964, page 64 .

[43] M. J. Madsen, W. K. Hensinger, D. Stick, J. A. Rabchuk, and C. Monroe, "Planar ion trap geometry for microfabrication", Appl. Phys. B 78, 639 (2004).

[44] D. Hucul et al., "On the transport of atomic ions in linear and multidimensinal ion trap arrays", Quant. Inf. Comp. 8, 501 (2008).

[45] P. M. Fitzpatrick, Advanced Calculus: A Course in Mathematical Analysis, PWS Publishing Company, 1st edition, 1996.

[46] K. Sugiyama and J. Yoda, "Production of $\mathrm{YbH}^{+}$by chemical reaction of $\mathrm{Yb}^{+}$in excited states with $\mathrm{H}_{2}$ gas", Phys. Rev. A 55, R10 (1997).

[47] K. Odaka and S. Ueda, "Dependence of outgassing rate on surface oxide layer thickness in type 304 stainless steel before and after surface oxidation in air", Vacuum 47, 689 (1996).

[48] C. D. Hodgman, R. C. Weast, and S. M. Selby, editors, Handbook of Chemistry and Physics, The Chemical Rubber Publishing Co., 43rd edition, 1961.

[49] W. W. Macalpine and R. O. Schildknecht, "Coaxial Resonators with Helical Inner Conductor", Proc. IRE , 2099 (1959).

[50] A. I. Zverev and H. J. Blinchikoff, "Realization of a Filter with Helical Components", IRE Trans. Compon. Parts Sept., 99 (1961).

[51] J. Cirac and P. Zoller, "Quantum Computations with Cold Trapped Ions", Phys. Rev. Lett. 74, 4091 (1995).

[52] R. G. DeVoe and C. Kurtsiefer, "Experimental study of anomalous heating and trap instabilities in a microscopic ${ }^{137} \mathrm{Ba}$ ion trap", Phys. Rev. A 65, 063407 (2002).

[53] M. R. Dietrich et al., "Barium Ions for Quantum Computation", in Proc. 9th Inter. Workshop on Non-Neutral Plasmas, 2008.

[54] C. Monroe, D. M. Meekhof, B. E. King, W. M. Itano, and D. J. Wineland, "Demonstration of a Fundamental Quantum Logic Gate", Phys. Rev. Lett. 75, 4714 (1995).

[55] J. Benhelm, G. Kirchmair, C. F. Roos, and R. Blatt, "Experimental quantum-information processing with ${ }^{43} \mathrm{Ca}^{+}$ions", Phys. Rev. A 77, 062306 (2008).

[56] J. Benhelm, G. Kirchmair, C. F. Roos, and R. Blatt, "Towards fault-tolerant quantum computing with trapped ions", Nature Physics 4, 463 (2008).

[57] J. P. Home et al., "Deterministic entanglement and tomography of ionspin qubits", New J. Phys. 8, 188 (2006). 
[58] P. J. Lee et al., "Atomic qubit manipulations with an electro-optic modulator", Op. Lett. 28, $1582(2003)$.

[59] M. D. Barrett et al., "Sympathetic cooling of ${ }^{9} \mathrm{Be}^{+}$and ${ }^{24} \mathrm{Mg}^{+}$for quantum logic", Phys. Rev. A 68, 042302 (2003).

[60] C. Balzer et al., "Electrodynamically trapped $\mathrm{Yb}^{+}$ions for quantum information processing", Phys. Rev. A 73, 041407(R) (2006).

[61] S. Olmschenk et al., "Manipulation and Detection of a Trapped $\mathrm{Yb}^{+}$Hyperfine Qubit", Phys. Rev. A 76, 052314 (2007).

[62] J. E. Sansonetti, W. C. Martin, and S. L. Young, Handbook of Basic Atomic Spectroscopic Data, Number 1.1.2, National Institute of Standards and Technology, Gaithersburg, MD, 2005, Available: http://physics.nist.gov/Handbook.

[63] C. Cabrillo, J. I. Cirac, P. García-Fernández, and P. Zoller, "Creation of entangled states of distant atoms by interference", Phys. Rev. A 59, 1025 (1999).

[64] C. Simon and W. T. M. Irvine, "Robust Long-Distance Entanglement and a Loophole-Free Bell Test with Ions and Photons", Phys. Rev. Lett. 91, 110405 (2003).

[65] L.-M. Duan, B. B. Blinov, D. L. Moehring, and C. Monroe, "Scalable Trapped Ion Quantum Computation with a Probabilistic Ion-Photon Mapping", Quant. Inf. Comp. 4, 165 (2004).

[66] L.-M. Duan et al., "Probabilistic quantum gates between remote atoms through interference of optical frequency qubits", Phys. Rev. A 73, 062324 (2006).

[67] J. Sherson, B. Julsgaard, and E. S. Polzik, "Deterministic atom-light quantum interface", Adv. At. Mol. Opt. Phys. 54, 82 (2006).

[68] J. Beugnon et al., "Quantum interference between two single photons emitted by independently trapped atoms", Nature 440, 779 (2006).

[69] P. Maunz et al., "Quantum interference of photon pairs from two remote trapped atomic ions", Nature Physics 3, 538 (2007).

[70] D. L. Moehring et al., "Entanglement of single-atom quantum bits at a distance", Nature 449, 68 (2007).

[71] T. Wilk, S. C. Webster, A. Kuhn, and G. Rempe, "Single-Atom Single-Photon Quantum Interface", Science 317, 488 (2007).

[72] S. Gerber et al., "Quantum interference from remotely trapped ions", New J. Phys. 11, $013032(2009)$.

[73] J. F. Poyatos, J. I. Cirac, R. Blatt, and P. Zoller, "Trapped ions in the strong-excitation regime: Ion interferometry and nonclassical states", Phys. Rev. A 54, 1532 (1996).

[74] J. J. García-Ripoll, P. Zoller, and J. I. Cirac, "Speed Optimized Two-Qubit Gates with Laser Coherent Control Techniques for Ion Trap Quantum Computing", Phys. Rev. Lett. 91, 157901 (2003).

[75] L.-M. Duan, "Scaling Ion Trap Quantum Computation through Fast Quantum Gates", Phys. Rev. Lett. 93, 100502 (2004).

[76] M. J. Madsen et al., "Ultrafast Coherent Coupling of Atomic Hyperfine and Photon Frequency Qubits", Phys. Rev. Lett. 97, 040505 (2006).

[77] T. Kuwamoto, K. Honda, Y. Takahashi, and T. Yabuzaki, "Magneto-optical trapping of Yb atoms using an intercombination transition", Phys. Rev. A 60, R745 (1999). 
[78] C. J. Bowers et al., "Experimental investigation of excited-state lifetimes in atomic ytterbium", Phys. Rev. A 53, 3103 (1996).

[79] M. Gustavsson, H. Lundberg, L. Nilsson, and S. Svanberg, "Lifetime measurements for excited states of rare-earth atoms using pulse modulation of a cw dye-laser beam", J. Opt. Soc. Am. 69, 984 (1979).

[80] C. W. Hoyt et al., "Observation and Absolute Frequency Measurements of the ${ }^{1} S_{0-}{ }^{3} P_{0}$ Optical Clock Transition in Neutral Ytterbium", Phys. Rev. Lett. 95, 083003 (2005).

[81] M. Roberts et al., "Measurement of the ${ }^{2} S_{1 / 2^{-}}{ }^{2} D_{5 / 2}$ clock transition in a single ${ }^{171} \mathrm{Yb}^{+}$ion", Phys. Rev. A 60, 2867 (1999).

[82] C. J. Foot, Atomic Physics, Oxford University Press Inc., 2005.

[83] H. J. Metcalf and P. van der Straten, Laser Cooling and Trapping, Springer-Verlag New York, Inc., 1999.

[84] D. J. Berkeland and M. G. Boshier, "Destabilization of dark states and optical spectroscopy in Zeeman-degenerate atomic systems", Phys. Rev. A 65, 033413 (2002).

[85] N. Yu and L. Maleki, "Lifetime measurements of the $4 f^{14} 5 d$ metastable states in single ytterbium ions", Phys. Rev. A 61, 022507 (2000).

[86] P. Taylor et al., "Investigation of the ${ }^{2} S_{1 / 2}-{ }^{2} D_{5 / 2}$ clock transition in a single ytterbium ion", Phys. Rev. A 56, 2699 (1997).

[87] R. W. Berends, E. H. Pinnington, B. Guo, and Q. Ji, "Beam-laser lifetime measurements for four resonance levels of Yb II", J. Phys. B 26, L701 (1993).

[88] M. Roberts, P. Taylor, G. P. Barwood, W. R. C. Rowley, and P. Gill, "Observation of the ${ }^{2} S_{1 / 2}{ }^{2} F_{7 / 2}$ electric octupole transition in a single ${ }^{171} \mathrm{Yb}^{+}$ion", Phys. Rev. A 62, 020501(R) (2000).

[89] E. Biémont, J.-F. Dutrieu, I. Martin, and P. Quinet, "Lifetime calculations in Yb II", J. Phys. B: At. Mol. Opt. Phys. 31, 3321 (1998).

[90] A. S. Bell, P. Gill, H. A. Klein, and A. P. Levick, "Laser cooling of trapped ytterbium ions using a four-level optical-excitation scheme", Phys. Rev. A 44, R20 (1991).

[91] H. Lehmitz, J. Hattendorf-Ledwoch, R. Blatt, and H. Harde, "Population trapping in excited Yb ions", Phys. Rev. Lett. 62, 2108 (1989).

[92] A. Bauch, D. Schnier, and C. Tamm, "Collisional population trapping and optical deexcitation of ytterbium ions in a radiofrequency trap", J. Mod. Opt. 39, 389 (1992).

[93] R. D. Cowan, The Theory of Atomic Structure and Spectra, University of California Press, 1981, Details of the level coupling notation can be found here, e.g. pages 128-132.

[94] B. C. Fawcett and M. Wilson, "Computed oscillator strengths, Landé $g$ values, and lifetimes in Yb II", Atomic Data and Nuclear Data Tables 47, 241 (1991).

[95] D. J. Berkeland, J. D. Miller, J. C. Bergquist, W. M. Itano, and D. J. Wineland, "Minimization of ion micromotion in a Paul trap", J. Appl. Phys. 83, 5025 (1998).

[96] R. W. P. Drever et al., "Laser Phase and Frequency Stabilization Using an Optical Resonator", Appl. Phys. B 31, 97 (1983).

[97] E. D. Black, "An introduction to Pound-Drever-Hall laser frequency stabilization", Am. J. Phys. 69, 79 (2001). 
[98] H. Ludvigsen and C. Holmlund, "Frequency stabilization of a GaAlAs semiconductor laser to an absorption line of iodine vapor", Rev. Sci. Inst. 63, 2135 (1992).

[99] S. Gerstenkorn, J. Verges, and J. Chevillard, Atlas du spectre d'asorption de la molecule d'iode, 11000-14000 cm-1, Laboratoire Aime-Cotton, CNRS II, 91405 Orsay, France, 1982.

[100] S. Gerstenkorn and P. Luc, Atlas du spectre d'absorption de la molecule d'iode, 14800-20000 $\mathrm{cm}^{-1}$, Centre National de la Recherche Scientifique, Paris, France, 1978.

[101] C. S. Edwards, G. P. Barwood, P. Gill, F. Rodriguez-Llorente, and W. R. C. Rowley, "Frequency-stabilised diode lasers in the visible region using Doppler-free iodine spectra", Op. Comm. 132, 94 (1996).

[102] P. Dubé and M. Trinczek, "Hyperfine-structure splittings and absorption strengths of molecular-iodine transitions near the trapping frequencies of francium", J. Opt. Soc. Am. $B$ 21, 1113 (2004).

[103] J. Migdalek, "Influence of core polarisation on relativistic oscillator strengths for lowest s-p transitions in Yb II-Hf IV spectra", J. Phys. B. 13, L169 (1980).

[104] A.-M. Mårtensson-Pendrill, D. S. Gough, and P. Hannaford, "Isotope shifts and hyperfine structure in the 369.4-nm $6 s-6 p_{1 / 2}$ resonance line of singly ionized ytterbium", Phys. Rev. A 49, 3351 (1994).

[105] B. B. Blinov, D. Leibfried, C. Monroe, and D. J. Wineland, "Quantum Computing with Trapped Ion Hyperfine Qubits", Quan. Inf. Proc. 3, 45 (2004).

[106] M. Acton et al., "Near-Perfect Simultaneous Measurement of a Qubit Register", Phys. Rev. A 65, 033413 (2002).

[107] M. L. Boas, Mathematical Methods in the Physical Sciences, John Wiley \& Sons, Inc., 2nd edition, 1983.

[108] C. K. Hong, Z. Y. Ou, and L. Mandel, "Measurement of Subpicosecond Time Intervals between Two Photons by Interference", Phys. Rev. Lett. 59, 2044 (1987).

[109] Y. H. Shih and C. O. Alley, "New Type of Einstein-Podolsky-Rosen-Bohm Experiment Using Pairs of Light Quanta Produced by Optical Parametric Down Conversion", Phys. Rev. Lett. 61, 2921 (1988).

[110] T. Legero, T. Wilk, A. Kuhn, and G. Rempe, "Time-resolved two-photon quantum interference", Appl. Phys. B 77, 797 (2003).

[111] J. D. Jackson, Classical Electrodynamics, John Wiley \& Sons, Inc., 3rd edition, 1999.

[112] S. J. van Enk, "Single-particle entanglement", Phys. Rev. A 72, 064306 (2005).

[113] A. Drezet, "Comment on 'Single-particle entanglement"', Phys. Rev. A 74, 026301 (2006).

[114] S. J. van Enk, "Reply to 'Comment on 'Single-particle entanglement"”, Phys. Rev. A 74, $026302(2006)$.

[115] Z. Yuan et al., "Electrically Driven Single-Photon Source", Science 295, 102 (2002).

[116] C. Tamm, D. Engelke, and V. Bühner, "Spectroscopy of the electric-quadrupole transition ${ }^{2} S_{1 / 2}(F=0){ }^{2} D_{3 / 2}(F=2)$ in trapped ${ }^{171} \mathrm{Yb}^{+} "$, Phys. Rev. A 61, 053405 (2000).

[117] P. J. Blythe et al., "Subkilohertz absolute-frequency measurement of the 467-nm electric octupole transition in ${ }^{171} \mathrm{Yb}^{+}$", Phys. Rev. A 67, 020501(R) (2003). 
[118] T. Schneider, E. Peik, and C. Tamm, "Sub-Hertz Optical Frequency Comparisons between Two Trapped ${ }^{171} \mathrm{Yb}^{+}$Ions", Phys. Rev. Lett. 94, 230801 (2005).

[119] U. I. Safronova, W. R. Johnson, M. S. Safronova, and J. R. Albritton, "Relativistic manybody calculations of excitation energies and transition rates in ytterbiumlike ions", Phys. Rev. A 66, 022507 (2002).

[120] C. R. Cowley, "Lanthanide Rare Earths in Stellar Spectra With Emphasis on Chemically Peculiar Stars", Physica Scripta T8, 28 (1984).

[121] N. Grevesse, "Accurate Atomic Data and Solar Photospheric Spectroscopy", Physica Scripta T8, 49 (1984).

[122] M. Reyniers and H. Van Winckel, "Detection of elements beyond the Ba-peak in VLT+UVES spectra of post-AGB stars", Astronomy 83 Astrophysics 408 (2003).

[123] K. B. Blagoev, V. A. Komarovskii, and N. P. Penkin, Opt. Spectrosc. (USSR) 45, 832 (1978).

[124] M. L. Burshtein, Y. F. Verolainen, V. A. Komarovskii, A. L. Osherovich, and N. P. Penkin, Opt. Spectrosc. (USSR) 37, 351 (1974).

[125] T. Andersen, O. Poulsen, P. S. Ramanujam, and A. Petrakiev Petkov, "Lifetimes of some excited states in the rare earths: La II, Ce II, Pr II, ND II, Sm II, Yb I, Yb II, and Lu II", Sol. Phys. 44, 257 (1975).

[126] F. H. K. Rambow and L. D. Schearer, "Radiative lifetimes and alignment depolarization cross sections for Yb I and II by the Hanle effect in a flowing helium system", Phys. Rev. A 14, 738 (1976).

[127] R. M. Lowe, P. Hannaford, and A.-M. Mårtensson-Pendrill, "Radiative lifetimes of the $6 p^{2} P_{1 / 2}^{0}$ and $6 p^{2} P_{3 / 2}^{0}$ levels in Yb II", Z. Phys. D 28, 283 (1993).

[128] Z. S. Li, S. Svanberg, P. Quinet, X. Tordoir, and E. Biémont, "Lifetime measurements in Yb II with time-resolved laser spectroscopy", J. Phys. B 32, 1731 (1999).

[129] E. H. Pinnington, G. Rieger, and J. A. Kernahan, "Beam-laser measurements of the lifetimes of the $6 p$ levels in Yb II", Phys. Rev. A 56, 2421 (1997).

[130] L. Young et al., "Precision lifetime measurements of Cs $6 p^{2} P_{1 / 2}$ and $6 p^{2} P_{3 / 2}$ levels by single-photon counting", Phys. Rev. A 50, 2174 (1994).

[131] D. L. Moehring et al., "Precision Lifetime Measurement of a Single Trapped Ion with Ultrafast Laser Pulses", Phys. Rev. A 73, 023413 (2006).

[132] R. G. DeVoe and R. G. Brewer, "Precision measurements of the lifetime of a single trapped ion with a nonlinear electro-optic switch", Op. Lett. 19, 1891 (1994).

[133] R. G. DeVoe and R. G. Brewer, "Observation of Superradiant and Subradiant Spontaneous Emission of Two Trapped Ions", Phys. Rev. Lett. 76, 2049 (1996).

[134] M. P. Silverman, S. Haroche, and M. Gross, "General theory of laser-induced quantum beats. I. Saturation effects of single laser excitation", Phys. Rev. A 18, 1507 (1978).

[135] E. Biémont et al., "Lifetime measurements and calculations in singly ionized ytterbium", $J$. Phys. B 35, 4743 (2002).

[136] U. I. Safronova and M. S. Safronova, "Third-order relativistic many-body calculations of energies, transition rates, hyperfine constants, and blackbody radiation shift in ${ }^{171} \mathrm{Yb}^{+} "$, Phys. Rev. A 79, 022512 (2009). 
[137] R. Raussendorf and H. J. Briegel, "A One-Way Quantum Computer", Phys. Rev. Lett. 86, 5188 (2001).

[138] P. Walther et al., "Experimental one-way quantum computing", Nature 434, 169 (2005).

[139] C.-Y. Lu et al., "Experimental entanglement of six photons in graph states", Nature Physics 3, 91 (2007).

[140] T. P. Bodiya and L.-M. Duan, "Scalable Generation of Graph-State Entanglement Through Realistic Linear Optics", Phys. Rev. Lett. 97, 143601 (2006).

[141] L.-M. Duan and R. Raussendorf, "Efficient Quantum Computation with Probabilistic Quantum Gates", Phys. Rev. Lett. 95, 080503 (2005).

[142] L.-M. Duan and C. Monroe, "Robust Quantum Information Processing with Atoms, Photons, and Atomic Ensembles", Adv. At. Mol. Opt. Phys. 55, 419 (2008).

[143] P. Maunz et al., "A heralded quantum gate between remote quantum memories", (2009), arXiv:quant-ph/0902.2136.

[144] S. L. Braunstein and A. Mann, "Measurement of the Bell operator and quantum teleportation", Phys. Rev. A 51, R1727 (1995).

[145] D. N. Matsukevich, P. Maunz, D. L. Moehring, S. Olmschenk, and C. Monroe, "Bell Inequality Violation with Two Remote Atomic Qubits", Phys. Rev. Lett. 100, 150404 (2008).

[146] D. F. V. James, P. G. Kwiat, W. J. Munro, and A. G. White, "Measurement of qubits", Phys. Rev. A 64, 052312 (2001).

[147] D. Gottesman and I. L. Chuang, "Demonstrating the viability of universal quantum computation using teleportation and single-qubit operations", Nature 402, 390 (1999).

[148] D. Bouwmeester et al., "Experimental quantum teleportation", Nature 390, 575 (1997).

[149] D. Boschi, S. Branca, F. De Martini, L. Hardy, and S. Popescu, "Experimental Realization of Teleporting an Unknown Pure Quantum State via Dual Classical and Einstein-PodolskyRosen Channels", Phys. Rev. Lett. 80, 1121 (1998).

[150] A. Furusawa et al., "Unconditional Quantum Teleportation", Science 282, 706 (1998).

[151] J. F. Sherson et al., "Quantum teleportation between light and matter", Nature 443, 557 (2006).

[152] Y.-A. Chen et al., "Memory-built-in quantum teleportation with photonic and atomic qubits", Nature Physics 4, 103 (2008).

[153] M. Riebe et al., "Deterministic quantum teleportation with atoms", Nature 429, 734 (2004).

[154] M. D. Barrett et al., "Deterministic quantum teleportation of atomic qubits", Nature 429, 737 (2004).

[155] M. Riebe et al., "Quantum teleportation with atoms: quantum process tomography", New J. Phys. 9, 211 (2007).

[156] S. Olmschenk et al., "Quantum Teleportation between Distant Matter Qubits", Science 323, 486 (2009).

[157] S. Massar and S. Popescu, "Optimal Extraction of Information from Finite Quantum Ensembles", Phys. Rev. Lett. 74, 1259 (1995).

[158] S. J. van Enk and N. Lütkenhaus and H. J. Kimble, "Experimental procedures for entanglement verification", Phys. Rev. A 75, 052318 (2007). 
[159] J. B. Altepeter, E. R. Jeffrey, and P. G. Kwiat, "Photonic State Tomography", Adv. At. Mol. Opt. Phys. 52, 105 (2006).

[160] J. L. O'Brien et al., "Quantum Process Tomography of a Controlled-NOT Gate", Phys. Rev. Lett. 93, 080502 (2004).

[161] M. Horodecki, P. Horodecki, and R. Horodecki, "General teleportation channel, singlet fraction, and quasidistillation", Phys. Rev. A 60, 1888 (1999).

[162] E. W. Streed, B. G. Norton, J. J. Chapman, and D. Kielpinski, "Scalable, efficient ionphoton coupling with phase Fresnel lenses for large-scale quantum computing", (2008), arXiv:0805.2437.

[163] G. Shu, M. R. Dietrich, N. Kurz, and B. B. Blinov, "Trapped Ion Imaging with a High Numerical Aperture Spherical Mirror", (2009), arXiv:0901.4742.

[164] N. Lindlein et al., "A New $4 \pi$ Geometry Optimized for Focusing on an Atom with a DipoleLike Radiation Pattern", Laser Physics 17, 927 (2007).

[165] R. Maiwald et al., "Ion traps with enhanced optical and physical access", (2009), arXiv:0810.2647.

[166] M. Hennrich, T. Legero, A. Kuhn, and G. Rempe, "Vacuum-Stimulated Raman Scattering Based on Adiabatic Passage in a High-Finesse Optical Cavity", Phys. Rev. Lett. 85, 4872 (2000).

[167] J. McKeever et al., "Deterministic Generation of Single Photons from One Atom Trapped in a Cavity", Science 303, 1992 (2004).

[168] I. Niven, H. S. Zuckerman, and H. L. Montgomery, An Introduction to the Theory of Numbers, John Wiley \& Sons, Inc., 5th edition, 1991.

[169] M. van Schilfgaarde, I. A. Abrikosov, and B. Johansson, "Origin of the Invar effect in ironnickel alloys", Nature 400, 46 (1999).

[170] G. R. Fowles, Introduction to Modern Optics, Dover Publications, Inc., 2nd edition, 1975. 